\title{
Scaling Relationships Based on Scaled Tank Mixing and Transfer Test Results
}

GF Piepel

A Heredia-Langner

KP Lee

SE Kelly

January 2014 


\title{
DISCLAIMER
}

This report was prepared as an account of work sponsored by an agency of the United States Government. Neither the United States Government nor any agency thereof, nor Battelle Memorial Institute, nor any of their employees, makes any warranty, express or implied, or assumes any legal liability or responsibility for the accuracy, completeness, or usefulness of any information, apparatus, product, or process disclosed, or represents that its use would not infringe privately owned rights. Reference herein to any specific commercial product, process, or service by trade name, trademark, manufacturer, or otherwise does not necessarily constitute or imply its endorsement, recommendation, or favoring by the United States Government or any agency thereof, or Battelle Memorial Institute. The views and opinions of authors expressed herein do not necessarily state or reflect those of the United States Government or any agency thereof.

\author{
PACIFIC NORTHWEST NATIONAL LABORATORY \\ operated by \\ BATTELLE \\ for the \\ UNITED STATES DEPARTMENT OF ENERGY \\ under Contract DE-AC05-76RL01830
}

Printed in the United States of America

Available to DOE and DOE contractors from the Office of Scientific and Technical Information

P.O. Box 62, Oak Ridge, TN 37831-0062;

ph: (865) 576-8401

fax: $(865) 576-5728$

email: reports $(\boldsymbol{a})$ adonis.osti.gov

Available to the public from the National Technical Information Service

5301 Shawnee Rd., Alexandria, VA 22312

ph: (800) 553-NTIS (6847)

email: orders $\alpha$ ntis.gov <http://www.ntis.gov/about/form.aspx >

Online ordering: http://www.ntis.gov

(3) This document was printed on recycled paper

(8/2010) 


\title{
Scaling Relationships Based on Scaled Tank Mixing and Transfer Test Results
}

\author{
GF Piepel ${ }^{1}$ \\ AE Holmes ${ }^{1}$ \\ A Heredia-Langner ${ }^{1}$ \\ KP Lee ${ }^{2}$ \\ SE Kelly ${ }^{2}$
}

January 2014

Prepared for

the U.S. Department of Energy

under Contract DE-AC05-76RL01830

\footnotetext{
1 Pacific Northwest National Laboratory Richland, Washington 99352

2 Washington River Protection Solutions Richland, Washington 99352
} 



\section{Executive Summary}

Washington River Protection Solutions (WRPS), as the Hanford Site tank farms contractor, will be responsible for transferring waste from a double-shell tank (DST) to the Waste Treatment and Immobilization Plant (WTP) when the WTP begins operations. The WRPS approach for predicting mixing and transfer performance (MTP) for a DST involves three primary steps:

1. Conduct tests consisting of variations in several parameters in two scaled tanks in the Small Scale Mixing Demonstration (SSMD) platform to generate data on MTP.

2. Develop scaling relationships for MTP as a function of the test parameters using the test data from Step 1.

3. Use the scaling relationships from Step 2 that are valid for the two test scales, as well as other knowledge and methods for mixing and transfer, to develop scaling relationships applicable to full-scale DST performance.

Step 1 has been completed by WRPS, which involved generating data for 26 test combinations performed in both of the two scaled tanks of the SSMD platform (for a total of 52 tests). The two tanks had diameters of 43.2 (referred to as 43 subsequently) and 120 inches, which are 1:21 and 1:8 relative to a full-scale DST, respectively. Other parameters that were varied in testing included mixer-jet nozzle velocity $(U)$, base simulant $(B S)$, supernatant viscosity $(S V),{ }^{(a)}$ and transfer-line capture velocity $(C V)$. The first 22 of the 26 test combinations conducted in both size tanks were selected using a statistical experimental design approach. An additional 4 test combinations performed at both scales used parameter combinations that are most relevant to expected operating conditions, and had not been tested previously. For each of the 52 tests, samples were collected pre-transfer and for each of five batch transfers. The samples were prepared and analyzed, with the results being the concentrations (lb/gal slurry) of the four solids components (gibbsite, stainless steel, sand, and $\mathrm{ZrO}_{2}$ ) in the base simulant.

Step 2 consists of building mathematical models for each of the two scale tanks that describe the MTP as a function of the test parameters. These models can then be used to calculate mixer-jet nozzle velocities where performance is equal between tank scales. These points of equal performance can be used to derive scaling relationships, which will allow estimating performance for full-scale DSTs. This report documents the statistical analyses associated with Step 2, which were performed by Pacific Northwest National Laboratory (PNNL) on data from the 52 tests conducted. Preliminary efforts focused on trying to model MTP metrics consisting of differences or ratios of $B S$-component concentrations for the five batch transfers relative to pre-transfer. However, depending on the solids component, there was little difference in component concentrations in batch transfers compared to pre-transfer for many to most of the tests. Hence, such differences and ratios of component concentrations were not useful MTP metrics because the effects of the test parameters "canceled out." Instead, models were developed to predict the natural logarithms of component concentrations $\left(\ln \left(C_{j}\right)\right.$ for the $j^{\text {th }}$ component) separately for

(a) In this document, "supernatant" refers to supernatant liquid, including the interstitial liquid in settled solids. Supernatant properties are quantified by dynamic viscosity and density. However, because the plan was to have supernatant density linearly related to supernatant viscosity, only supernatant viscosity was used as a test parameter. 
the 43-inch and 120-inch tanks as functions of the test parameters $[U, B S$, kinematic viscosity $(K V)]$, ${ }^{\text {a) }}$ $C V$, and batch number $(B N) . B N$ is 0 for pre-transfer and 1-5 for the batch transfers. The reasons for modeling $\ln \left(C_{j}\right)$ are discussed in the opening discussion of Section 5.

Two model forms were investigated, which are listed as Equations (5.5) and (5.8). Equation (5.5) assumes the dependence of $\ln \left(C_{j}\right)$ on $U$ is adequately approximated by up to a cubic polynomial in $U$. Equation (5.8) assumes that the $\ln \left(C_{j}\right)$ versus $U$ relationship is adequately approximated by up to a quadratic polynomial in $\ln (U)$, which is based on a linearization of a power-law model and allowance for quadratic behavior. Stepwise regression was used to select the subset of terms in Equation (5.5) and Equation (5.8) that were statistically significant, with the exception that some lower-order terms were included in the models to maintain model hierarchy. Table S.1 summarizes for each model (i) the $\mathrm{R}^{2}$ value (fraction of variation in $\ln \left(C_{j}\right)$ values accounted for by the model), and (ii) whether the model has a statistically significant lack-of-fit (LOF) at the $95 \%$ confidence level. A statistically significant LOF occurs when the variation in the data not accounted for by the model is substantively larger than the variation in replicate tests. Table S.1 shows that (i) the models obtained from Equations (5.5) and (5.8) have similar $\mathrm{R}^{2}$ values and LOF results and (ii) with one exception the models do not have statistically significant LOFs. The exception is the model based on Equation (5.8) for SS concentration in the 120inch tank. However, the $\mathrm{R}^{2}$ is large enough $(>0.90)$ for that model to be used for the purposes of this report.

The sensitivity of the model-predicted component concentrations to changes in $U, B S, K V$, and $C V$ are assessed graphically in Section 7.4 using prediction profile plots (PPPs). Models based on Equation (5.5) with up to cubic dependence on $U$ were found to sometimes significantly overpredict SS concentrations (compared to the range of values observed in testing) for combinations of the test parameters within their test ranges. This overprediction behavior is undesirable and led to the decision not to recommend use of those models. Table S.2 summarizes the order of importance of the parameters in predicting component concentrations, for all four base-simulant components with both kinds of models. It is not surprising that $B S$ often appears as the most important predictor of component concentration because the mass fraction of each component used in each test run was determined by the type of base simulant.

The $\ln \left(C_{j}\right)$ models of both kinds were used with an equivalent performance (EP) approach (discussed in Section 6.2) to calculate scaling exponents (denoted " $a$ ") based on a geometric scaling equation derived by assuming that $C_{j}$ has a power-law relationship with $U$ and tank diameter. The EP approach involves (i) creating a grid of $B S, K V, C V$, and $B N$ value combinations, (ii) generating for each grid combination a sequence of mixer-jet nozzle velocities for the 43-inch tank $\left(U_{43}\right)$, and (iii) calculating for each $U_{43}$ the mixer-jet nozzle velocity for the 120-inch tank $\left(U_{120}\right)$ that gives EP, which for this work was equal component concentrations (lb/gal slurry). Thus for every grid combination of $B S, K V, C V$, and $B N$, there was a series of $\left(U_{43}, U_{120}\right)$ pairs that yield EP (equal concentrations), which were then used to calculate " $a$ " values. The sensitivities of the " $a$ " values to $U_{43}, B S, K V, C V$, and $B N$ were then assessed graphically for both kinds of component-concentration models. Scaling components could not be calculated using the EP approach if either the 43-inch or 120-inch models for a given component did not depend on $U$. This was the case for gibbsite (both kinds of model) and $\mathrm{ZrO}_{2}$ (the second kind of model), as shown in Tables S.1 and S.2.

(a) Kinematic viscosity (dynamic viscosity divided by density) was used for modeling as is traditional, and also because there was not an exact linear relationship between supernatant viscosity and density as envisioned.. 
Table S.1. $R^{2}$ Values and Model Lack-of-Fit Assessment for Stepwise Regression Fits of Natural Logarithm of Component Concentrations Based on Models (5.5) and (5.8), Separately for Each of the Two Scaled Tanks

\begin{tabular}{|c|c|c|c|c|}
\hline $\begin{array}{c}\text { Tank } \\
\text { Diameter } \\
\text { (inches) }\end{array}$ & $\ln ($ Component $)$ & Model Equation & $\mathbf{R}^{2(a)}$ & $\begin{array}{c}\text { LOF Significant } \\
\text { with } 95 \% \text { Confidence }\end{array}$ \\
\hline \multirow{8}{*}{43} & \multirow{2}{*}{$\ln$ (gibbsite) } & $(5.5)$ & 0.9940 & No \\
\hline & & $(5.8)$ & 0.9946 & No \\
\hline & \multirow{2}{*}{$\ln ($ stainless steel) } & $(5.5)$ & 0.9318 & No \\
\hline & & $(5.8)$ & 0.9350 & No \\
\hline & \multirow{2}{*}{$\ln ($ sand $)$} & $(5.5)$ & 0.9784 & No \\
\hline & & $(5.8)$ & 0.9785 & No \\
\hline & \multirow{2}{*}{$\ln \left(\mathrm{ZrO}_{2}\right)$} & $(5.5)$ & 0.8151 & No \\
\hline & & $(5.8)$ & 0.7652 & No \\
\hline \multirow{8}{*}{120} & \multirow{2}{*}{$\ln$ (gibbsite) } & $(5.5)$ & 0.9916 & No \\
\hline & & $(5.8)$ & 0.9916 & No \\
\hline & \multirow{2}{*}{$\ln ($ stainless steel) } & $(5.5)$ & 0.9323 & No \\
\hline & & $(5.8)$ & 0.9074 & Yes \\
\hline & \multirow{2}{*}{$\ln ($ sand $)$} & $(5.5)$ & 0.9551 & No \\
\hline & & $(5.8)$ & 0.9552 & No \\
\hline & \multirow{2}{*}{$\ln \left(\mathrm{ZrO}_{2}\right)$} & $(5.5)$ & 0.6906 & No \\
\hline & & $(5.8)$ & 0.6469 & No \\
\hline
\end{tabular}

Table S.2. Sensitivity of Model-Predicted Component Concentrations to Model Parameters

\begin{tabular}{lcll}
\hline & $\begin{array}{c}\text { Tank } \\
\text { Diameter } \\
\text { Component }\end{array}$ & \multicolumn{2}{c}{$\begin{array}{c}\text { Parameters, from Most to Least Important Regarding } \\
\text { Effects on Component Concentrations }\end{array}$} \\
\cline { 3 - 4 } (in.) & $\begin{array}{l}\text { Models with Up to } \\
\text { Cubic Dependence on } \boldsymbol{U}\end{array}$ & $\begin{array}{l}\text { Models with Up to } \\
\text { Quadratic Dependence on } \ln (\boldsymbol{U})\end{array}$ \\
\hline Gibbsite & 43 & $B S$ & $B S, U, K V$ \\
Gibbsite & 120 & $B S, C V, K V$ & $B S, C V, K V$ \\
SS & 43 & $B S, U, K V, C V$ & $U, B S, K V, C V$ \\
SS & 120 & $B S, U, K V, C V$ & $B S, U, K V, C V$ \\
Sand & 43 & $B S, U, K V$ & $B S, U, K V$ \\
Sand & 120 & $B S, C V, U, K V$ & $B S, C V, U, K V$ \\
$Z \mathrm{ZrO}_{2}$ & 43 & $U, B S, K V, C V$ & $B S, K V, U, C V$ \\
$\mathrm{ZrO}_{2}$ & 120 & $U, B S, C V, K V$ & $B S, C V, K V$ \\
\hline
\end{tabular}

Table S.3 summarizes the ranges of " $a$ " for SS and sand concentrations using both kinds of models. The much larger range of scaling exponent values for the Section 7.1 models is related to modeling component concentrations using up to cubic dependence on $U$. The PPPs presented and discussed in Section 7.4.1 showed that these models for SS concentrations could significantly overpredict component concentrations in some cases. On the other hand, much smaller scaling exponent values are obtained using the Section 7.2 models, apparently due to modeling component concentrations as functions of $\ln (U)$ and possibly $[\ln (U)]^{2}$. The PPPs presented and discussed in Section 7.4.2 did not show the same tendency for the Section 7.2 models to overpredict SS concentrations. 
Table S.3. Summary of Scaling Exponent Ranges for SS and Sand Concentrations Based on the Models with Dependence on $U$ or $\ln (U)$ Discussed in Sections 7.1 and 7.2, Respectively

\begin{tabular}{llcc}
\hline & & \multicolumn{2}{c}{ Range of All Scaling Exponents } \\
\hline Component & BS & Section 7.1 Models & Section 7.2 Models \\
\hline SS & Typical & {$[-0.195,0.771]$} & {$[-0.187,0.321]$} \\
SS & High & {$[-0.136,0.688]$} & {$[-0.067,0.319]$} \\
Sand & Typical & {$[-0.052,0.262]$} & {$[-0.054,0.261]$} \\
Sand & High & {$[-0.052,0.345]$} & {$[-0.052,0.343]$} \\
\hline
\end{tabular}

Based on the PPPs in Section 7.4 and the scaling exponents discussed in Sections 8.1 and 8.2, it is concluded that the models in Section 7.2 are preferred for modeling component concentrations and for calculating scaling exponents using the EP approach discussed in Section 6.2. The maximum scaling exponents calculated by the Section 7.2 models for SS and sand are within the [0.2-0.4] range envisioned by Lee (2012, p. 3-27). Also, the range of scaling exponent values calculated by the Section 7.2 models is consistent with values anticipated based on Figures 3.2 and 3.3. Hence, it is recommended that the range of scaling exponents identified using the Section 7.2 models be explored by WRPS in Step 3 (at the start of the Executive Summary) to estimate full-scale, DST performance. It can be concluded that scaling exponent is a function of jet velocity and simulant make-up (e.g. $B S, K V$ ). Therefore all these factors must be considered when estimating full-scale performance. 


\section{Acknowledgments}

The authors thank Mike Thien, Raphael Milleret, and Ted Wooley (Washington River Protection Solutions) for their support and guidance, on this work. Discussions with Phil Gauglitz, Beric Wells, and James Fort of Pacific Northwest National Laboratory (PNNL) have also been extremely helpful. The authors also thank several PNNL staff: Paul Eslinger for reviewing the report with a focus on the statistical content, Jim Fort for reviewing the report with a focus on the mixing and transfer aspects, and Andrea Currie for editing the report. The work documented in this report was sponsored by WRPS and conducted by the PNNL coauthors based on WRPS-supplied data and collaborative interactions with the WRPS coauthors. PNNL is operated by Battelle for the U.S. Department of Energy under Contract DEAC05-76RL01830. 



\section{Quality Assurance}

The work in this report was conducted according to the "Quality Assurance Plan for Statistical Support of Tank Farms Mixing and Sampling” (QA-63993-001_R0) by D.B. MacPherson, December 2012. That plan allowed for some flexibility in using the quality assurance (QA) procedures listed for guidance, because those procedures involve a higher level of QA than required by Washington River Protection Solutions (WRPS) for this work. A formal QA package was prepared, which documents the data supplied by WRPS as well as the statistical analyses and software calculations that yielded the key results presented in this report. Some figures in the report merely graphically display data, while some tables summarize the uncertainties in the data based on replicate tests. Such figures and tables were carefully reviewed and checked by the person performing the work. Because of their simplicity, such figures and tables were not included in the formal QA package and were not independently reviewed. The figures and tables in this category are marked "For Information Only" at the end of a figure or table title. The work to produce the figures and tables not marked as "For Information Only" was documented in the formal QA package and independently reviewed. 



\section{Acronyms and Abbreviations}

\begin{tabular}{|c|c|}
\hline $\mathrm{Al}(\mathrm{OH})_{3}$ & gibbsite \\
\hline$B N$ & batch number \\
\hline$B S$ & base simulant \\
\hline $\mathrm{cP}$ & centipoise \\
\hline $\mathrm{cSt}$ & centistokes \\
\hline$C V$ & transfer-line capture velocity \\
\hline$D$ & diameter \\
\hline DST & double-shell tank \\
\hline EP & equivalent performance \\
\hline $\mathrm{ft}$ & foot, feet \\
\hline $\mathrm{g} / \mathrm{mL}$ & grams per milliliter \\
\hline gpm & gallons per minute \\
\hline in. & inch(es) \\
\hline$K V$ & kinematic viscosity \\
\hline lb/gal & pounds per gallon \\
\hline $\ln$ & natural logarithm \\
\hline LOF & lack of fit \\
\hline $\mathrm{mL}$ & milliliter(s) \\
\hline MTP & mixing and transfer performance \\
\hline OLS & ordinary least squares \\
\hline PNNL & Pacific Northwest National Laboratory \\
\hline PPP & prediction profile plot \\
\hline PvM & predicted versus measured \\
\hline $\mathrm{R}^{2}$ & $\begin{array}{l}\text { fraction of the variation in the data values of a property (or mathematical } \\
\text { transformation thereof) that is accounted for by a model }\end{array}$ \\
\hline $\mathrm{R}_{\mathrm{A}}^{2}$ & $\begin{array}{l}\text { fraction of the variation in the data values of a property (or mathematical } \\
\text { transformation thereof) in the model, adjusted for the number of coefficients and } \\
\text { data points, that is accounted for by a model }\end{array}$ \\
\hline RMSE & root mean squared error \\
\hline$\%$ RSD & percent relative standard deviation \\
\hline RSD & relative standard deviation \\
\hline $\mathrm{SD}$ & standard deviation \\
\hline $\sec$ & second $(s)$ \\
\hline $\mathrm{SiO}_{2}$ & sand \\
\hline SS & stainless steel \\
\hline SSE & sum of squared errors \\
\hline
\end{tabular}


SSMD

SV

$U$

WRPS

WTP

$\mathrm{wt} \%$

$\mathrm{ZrO}_{2}$
Small Scale Mixing Demonstration

supernatant viscosity

mixer-jet nozzle velocity

Washington River Protection Solutions

Hanford Tank Waste Treatment and Immobilization Plant

weight percent

zirconium dioxide 


\section{Contents}

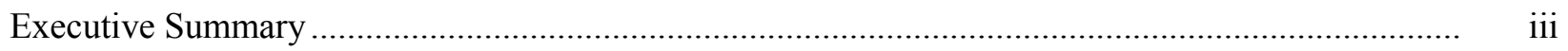

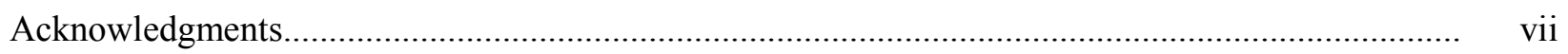

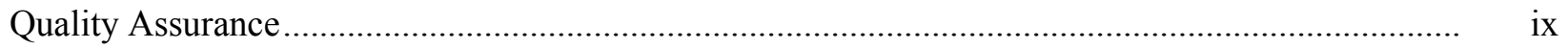

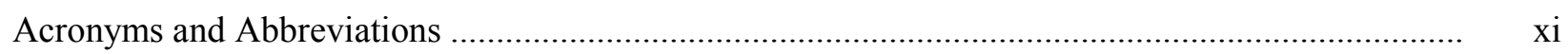

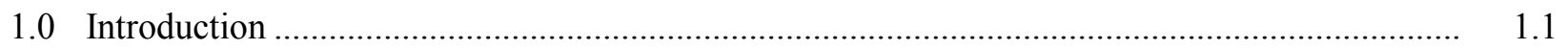

2.0 Small Scale Mixing Demonstration Scaling Performance Tests ............................................ 2.1

2.1 Experimental Parameters and Possible Settings.......................................................... 2.1

2.2 Experimental Design of Tests at Both Scales ................................................................ 2.3

2.3 Four Additional Test Combinations …...................................................................... 2.6

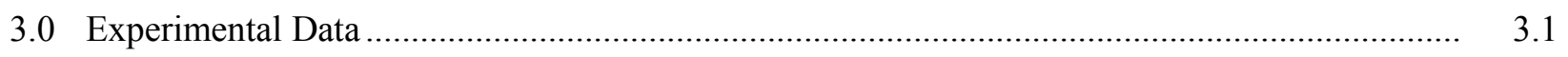

3.1 Collection, Preparation, and Chemical Analysis of Samples .......................................... 3.1

3.1.1 Collection of Initial Samples ........................................................................... 3.1

3.1.2 Preparation of Samples for Chemical Analyses ................................................ 3.1

3.1.3 Laboratory Chemical Analyses ...................................................................... 3.2

3.1.4 Normalization of Analyzed Component Weight Percent Values.............................. 3.3

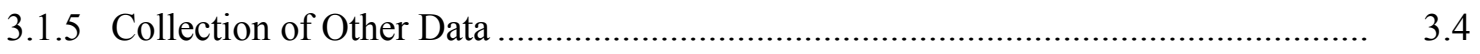

3.2 Experimental Data for the 26 Test Combinations ........................................................ 3.4

3.3 Graphical Assessment of Component Concentrations Data............................................ 3.7

3.3.1 Component Concentrations Plotted Versus Pre-Transfer and Transfer Batches....... $\quad 3.7$

3.3.2 Component Concentrations Plotted Versus Mixer-Jet Nozzle Velocity ................... 3.8

3.4 Estimates of Uncertainties in Component Concentrations Based on Replicate Tests......... 3.12

4.0 Mixing and Transfer Performance ............................................................................. 4.1

4.1 Possible Performance Measures ....................................................................................... 4.1

4.2 Graphical Assessment of Performance Measures ........................................................... 4.2

5.0 Models for Component Concentrations as Functions of Test Parameters ................................ 5.1

5.1 Models for Component Concentrations as Functions of the Test Parameters with a Cubic Polynomial Dependence on Jet Velocity ............................................................. 5.2

5.1.1 Polynomial Model Form for Component Concentrations for Each Tank and PreTransfer or Batch Transfer ............................................................................. 5.2

5.1.2 Models for Component Concentrations as Functions of the Test Parameters with a Cubic Polynomial Dependence on Jet Velocity ................................................ 5.6

5.2 Models for Component Concentrations as Functions of the Test Parameters with a Power Law Dependence on Jet Velocity..................................................................... 5.8

5.3 Models for Component Concentrations as Functions of the Test Parameters and Tank Diameter......

5.4 Model Fitting, Model Evaluation, and Sensitivity Assessment ..................................... 5.12

5.4.1 Stepwise Regression................................................................................. 5.12

5.4.2 Evaluating How Well a Model Fits the Data........................................................ 5.13 
5.4.3 Assessing the Sensitivity of Component Concentrations to Variations in the Test Parameters

6.0 Equivalent Performance Approach to Calculating Scaling Exponents................................... 6.1

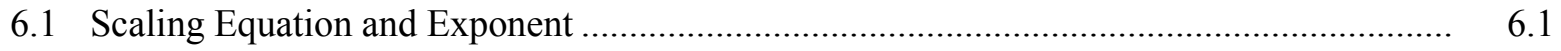

6.2 Equivalent Performance Approach to Calculating Scaling Exponents ............................. 6.2

7.0 Results of Modeling Base-Simulant Component Concentrations as Functions of Several Parameters

7.1 Results of Fitting Models for Component Concentrations as Functions of the Test Parameters with Up to a Cubic Polynomial Dependence on Jet Velocity

7.1.1 Models for Gibbsite Concentrations as Functions of the Test Parameters with Up to a Cubic Polynomial Dependence on Jet Velocity

7.1.2 Models for Stainless Steel Concentrations as Functions of the Test Parameters with Up to a Cubic Polynomial Dependence on Jet Velocity

7.1.3 Models for Sand Concentrations as Functions of the Test Parameters with Up to a Cubic Polynomial Dependence on Jet Velocity

7.1.4 Models for $\mathrm{ZrO}_{2}$ Concentrations as Functions of the Test Parameters with Up to a Cubic Polynomial Dependence on Jet Velocity

7.2 Results of Fitting Models for Component Concentrations as Functions of the Test Parameters with a Linearization and Quadratic Extension of a Power-Law Dependence on Jet Velocity.....

7.2.1 Models for Gibbsite Concentrations as Functions of the Test Parameters with a Linearization and Quadratic Extension of a Power-Law Dependence on Jet Velocity

7.2.2 Models for Stainless Steel Concentrations as Functions of the Test Parameters with a Linearization and Quadratic Extension of a Power-Law Dependence on Jet Velocity......

7.2.3 Models for Sand Concentrations as Functions of the Test Parameters with a Linearization and Quadratic Extension of a Power-Law Dependence on Jet Velocity.

7.2.4 Models for $\mathrm{ZrO}_{2}$ Concentrations as Functions of the Test Parameters with a Linearization and Quadratic Extension of a Power-Law Dependence on Jet Velocity

7.3 Comparisons of Fitted Models from the Two Approaches

7.4 Sensitivity of Model-Predicted Component Concentrations to Parameters in the Models.

7.4.1 Prediction Profile Plots for the Stainless Steel and Sand Concentration Models in Section 7.1

7.4.2 Prediction Profile Plots for the Stainless Steel and Sand Concentration Models in Section 7.2.

7.4.3 Comparison of Prediction Profile Plots for Stainless Steel and Sand Produced Using the Models from Section 7.1 and Section 7.2

8.0 Scaling Exponents as Functions of Test Parameters Calculated Using the Equivalent Performance Approach. 
8.1 Scaling Exponent Results Using Models for Component Concentrations as Functions of the Test Parameters with Up to a Cubic Polynomial Dependence on Jet Velocity

8.1.1 Scaling Exponent Results for Stainless Steel Concentrations Using Models with Up to a Cubic Polynomial Dependence on Jet Velocity

8.1.2 Scaling Exponent Results for Sand Concentrations Using Models with Up to a Cubic Polynomial Dependence on Jet Velocity

8.1.3 Scaling Exponent Results for $\mathrm{ZrO}_{2}$ Concentrations Using Models with Up to a Cubic Polynomial Dependence on Jet Velocity....

8.2 Scaling Exponent Results Using Models for Component Concentrations as Functions of the Test Parameters with a Power-Law Dependence on Jet Velocity.

8.2.1 Scaling Exponent Results for Stainless Steel Concentrations Using Models Related to a Power-Law Dependence on Jet Velocity .....

8.2.2 Scaling Exponent Results for Sand Concentrations Using Models Related to a Power-Law Dependence on Jet Velocity .....

8.3 Scaling Exponent Conclusions

Appendix A - Mixing and Transfer Performance Tests and Resulting Concentrations of Gibbsite, Stainless Steel, Sand, and $\mathrm{ZrO}_{2}$ in Pre-Transfer and Batch Transfer Samples.....

Appendix B - Plots of Component Concentrations from Pre-Transfer Samples and Samples from Five Batch Transfers for 26 Test Combinations Performed at Two Scales.

Appendix C - Plots of Standard Deviations and Percent Relative Standard Deviations of Component Concentrations Estimated from Replicate Tests

\section{Figures}

2.1 Subregion of Permissible Combinations of Supernatant Viscosity and Transfer-Line Capture Velocity

3.1 Plots of Gibbsite Concentrations versus Mixer-Jet Nozzle Velocity for 43-inch and 120inch Tanks for Seven Test Combinations with at Least Two Velocities

3.2 Plots of Stainless Steel Concentrations versus Mixer-Jet Nozzle Velocity for 43-inch and 120-inch Tanks for Seven Test Combinations with at Least Two Velocities .....

3.3 Plots of Sand Concentrations versus Mixer-Jet Nozzle Velocity for 43-inch and 120-inch Tanks for Seven Test Combinations with at Least Two Velocities

3.4 Plots of $\mathrm{ZrO}_{2}$ Concentrations versus Mixer-Jet Nozzle Velocity for 43-inch and 120-inch Tanks for Seven Test Combinations with at Least Two Velocities

4.1 Gibbsite Concentration Differences for 26 Test Combinations Plotted versus Batch Transfer Number for 43 -inch and 120-inch Tanks.

4.2 Stainless Steel Concentration Differences for 26 Test Combinations Plotted versus Batch Transfer Number for 43-inch and 120-inch Tanks.

4.3 Sand Concentration Differences for 26 Test Combinations Plotted versus Batch Transfer Number for 43-inch and 120-inch Tanks. 
4.4 $\mathrm{ZrO}_{2}$ Concentration Differences for 26 Test Combinations Plotted versus Batch Transfer Number for 43-inch and 120-inch Tanks .....

5.1 Plot of the Natural Logarithm of Actual Dynamic Viscosity of Supernatants versus Actual Bottom Density for the 22 Test Combinations in Table 2.3 and the 4 Additional Test Combinations in Table 2.4

5.2 Plot of Kinematic Viscosity $(K V)$ versus Dynamic Viscosity $(S V)$ for the Supernatants of the 22 Test Combinations in Table 2.3 and the 4 Additional Test Combinations in Table 2.4 ..

7.1 Predicted versus Measured Plots for the Gibbsite Concentration Models from Table 7.1 for the 43-inch Tank and 120-inch Tank

7.2 Predicted versus Measured Plots for the Stainless Steel Concentration Models from Table 7.2 for the 43-inch Tank and 120-inch Tank.

7.3 Predicted versus Measured Plots for the Sand Concentration Models from Table 7.3 for the 43-inch Tank and 120-inch Tank

7.4 Predicted versus Measured Plots for the $\mathrm{ZrO}_{2}$ Concentration Models from Table 7.4 for the 43-inch Tank and 120-inch Tank

7.5 Predicted versus Measured Plots for the Gibbsite Concentration Models from Table 7.5 for the 43-inch Tanks and 120-inch Tank.

7.6 Predicted versus Measured Plots for the Stainless Steel Concentration Models from Table 7.6 for the 43-inch Tank and 120-inch Tank.

7.7 Predicted versus Measured Plots for the Sand Concentration Models from Table 7.7 for the 43-inch Tank and 120-inch Tank

7.8 Predicted versus Measured Plots for the $\mathrm{ZrO}_{2}$ Concentration Models from Table 7.8 for the 43-inch Tank and 120-inch Tank

7.9. Prediction Profile Plot of Stainless Steel Concentrations versus Velocity Using the Models from Table 7.2 for Batch 1 from the 43-Inch and 120-Inch Tanks with Typical Base Simulant and Various Combinations of Kinematic Viscosity and Capture Velocity

7.10 Prediction Profile Plot of Stainless Steel Concentrations versus Velocity Using the Models from Table 7.2 for Batch 1 from the 43-Inch and 120-Inch Tanks with High Base Simulant and Various Combinations of Kinematic Viscosity and Capture Velocity

7.11 Prediction Profile Plot of Sand Concentrations versus Velocity Using the Models from Table 7.3 for Batch 1 from the 43-Inch and 120-Inch Tanks with Typical Base Simulant and Various Combinations of Kinematic Viscosity and Capture Velocity.

7.12 Prediction Profile Plot of Sand Concentrations versus Velocity Using the Models from Table 7.3 for Batch 1 from the 43-Inch and 120-Inch Tanks with High Base Simulant and Various Combinations of Kinematic Viscosity and Capture Velocity...

7.13 Prediction Profile Plot of Stainless Steel Concentrations versus Velocity Using the Model from Table 7.2 for Batch 1 from the 43-Inch and 120-Inch Tanks with Typical Base Simulant and Various Combinations of Kinematic Viscosity and Capture Velocity

7.14 Prediction Profile Plot of Stainless Steel Concentrations versus Velocity Using the Model from Table 7.2 for Batch 1 from the 43-Inch and 120-Inch Tanks with High Base Simulant and Various Combinations of Kinematic Viscosity and Capture Velocity

7.15 Prediction Profile Plot of Sand Concentrations versus Velocity Using the Model from Table 7.2 for Batch 1 from the 43-Inch and 120-Inch Tanks with Typical Base Simulant and Various Combinations of Kinematic Viscosity and Capture Velocity..... 
7.16 Prediction Profile Plot of Sand Concentrations versus Velocity Using the Model from Table 7.2 for Batch 1 from the 43-Inch and 120-Inch Tanks with High Base Simulant and Various Combinations of Kinematic Viscosity and Capture Velocity....

7.17 Comparison of Stainless Steel Prediction Profile Plots for Typical Base Simulant Produced Using the Models in Tables 7.2 and 7.6.

7.18 Comparison of Stainless Steel Prediction Profile Plots for Typical Base Simulant Produced Using the Models in Tables 7.2 and 7.6

7.19 Comparison of Sand Prediction Profile Plots for Typical Base Simulant Produced Using the Models in Tables 7.3 and 7.7

7.20 Comparison of Sand Prediction Profile Plots for Typical Base Simulant Produced Using the Models in Tables 7.3 and 7.7

8.1 Scaling Exponent Values for Stainless Steel versus Mixer-Jet Nozzle Velocity in the 43inch Tank Using the Models in Table 7.2

8.2 Scaling Exponent Values for Stainless Steel versus Mixer-Jet Nozzle Velocity in the 43inch Tank with $B S=$ Typical and Various Combinations of $K V$ and $C V$ Using the Models in Table 7.2.

8.3 Scaling Exponent Values for Stainless Steel versus Mixer-Jet Nozzle Velocity in the 43inch Tank with $B S=$ High and Various Combinations of $K V$ and $C V$ Using the Models in Table 7.2.

8.4 Scaling Exponent Values for Sand versus Mixer-Jet Nozzle Velocity in the 43-inch Tank Using the Models in Table 7.3....

8.5 Scaling Exponent Values for Sand versus Mixer-Jet Nozzle Velocity in the 43-inch Tank with $B S=$ Typical and Various Combinations of $K V$ and $C V$ Using the Models in Table 7.3.

8.6 Scaling Exponent Values for Sand versus Mixer-Jet Nozzle Velocity in the 43-inch Tank with $B S=$ High and Various Combinations of $K V$ and $C V$ Using the Models in Table 7.3

8.7 Scaling Exponent Values versus Mixer-Jet Nozzle Velocity in the 43-inch Tank for $\mathrm{ZrO}_{2}$ Using the Models in Table 7.4

8.8 Scaling Exponent Values for $\mathrm{ZrO}_{2}$ versus Mixer-Jet Nozzle Velocity in the 43-inch Tank with $B S=$ Typical and Various Combinations of $K V$ and $C V$ Using the Models in Table 7.4 .....

8.9 Scaling Exponent Values for $\mathrm{ZrO}_{2}$ versus Mixer-Jet Nozzle Velocity in the 43-inch Tank with $B S=$ High and Various Combinations of $K V$ and $C V$ Using the Models in Table 7.4.

8.10 Scaling Exponent Values versus Mixer-Jet Nozzle Velocity in the 43-inch Tank for Stainless Steel using the Models in Table 7.6.

8.11 Scaling Exponent Values versus Mixer-Jet Nozzle Velocity in the 43-inch Tank for Stainless Steel using the Models in Table 7.6 for $B S=$ Typical

8.12 Scaling Exponent Values versus Mixer-Jet Nozzle Velocity in the 43-inch Tank for Stainless Steel using the Models in Table 7.6 for $B S=$ High

8.13 Scaling Exponent Values versus Mixer-Jet Nozzle Velocity in the 43-inch Tank for Sand Using the Models in Table 7.7.

8.14 Scaling Exponent Values versus Mixer-Jet Nozzle Velocity in the 43-inch Tank for Sand Using the Models in Table 7.7 for $B S=$ Typical. 
8.15 Scaling Exponent Values versus Mixer-Jet Nozzle Velocity in the 43-inch Tank for Sand Using the Models in Table 7.7 for BS = High

\section{Tables}

S.1 Model Terms, $\mathrm{R}^{2}$ Values, and Model Lack-of-Fit Assessment for Stepwise Regression Fits of Natural Logarithm of Component Concentrations in Models 5.5 and 5.8, Separately for Each of the Two Scaled Tanks

S.2 Sensitivity of Model-Predicted Component Concentrations to Model Parameters.

S.3 Summary of Scaling Exponent Ranges for SS and Sand Concentrations Based on the Models with Dependence on U or ln Discussed in Sections 7.1 and 7.2, Respectively.

2.1 Test Parameters and Possible Settings for Scaled Performance Testing

2.2 Particulate Characteristics and Compositions of Typical and High Base Simulants

2.3 Experimental Design for Each of Two Tank Scales in SSMD Testing

2.4 Additional Four Test Combinations for Each of Two Tank Scales in SSMD Testing..

2.5 Target Mixer-Jet Pump Velocities

3.1 Minimum and Maximum Planned and Actual Values of Mixer-Jet Nozzle Velocity, Supernatant Viscosity, Capture Velocity, and Actual Values of Kinematic Viscosity

3.2 Minimum and Maximum Component Concentration Values Over the Pre-Transfer and Five Batch Transfer Values for Each Test and Over the 26 Test Combinations for Each Scaled Tank Separated by Base Simulant type...

3.3 Combinations of Base Simulant, Supernatant Viscosity, and Capture Velocity Tested at Two or More Mixer-Jet Nozzle Velocities.

3.4 Gibbsite Concentrations, Standard Deviations, and Percent Relative Standard Deviations for Pre-Transfer and Batch-Transfer Samples from Replicate Tests.

3.5 Stainless Steel Concentrations, Standard Deviations, and Percent Relative Standard Deviations for Pre-Transfer and Batch-Transfer Samples from Replicate Tests

3.6 Sand Concentrations, Standard Deviations, and Percent Relative Standard Deviations for Pre-Transfer and Batch-Transfer Samples from Replicate Tests.....

3.7 $\mathrm{ZrO}_{2}$ Concentrations, Standard Deviations, and Percent Relative Standard Deviations for Pre-Transfer and Batch-Transfer Samples from Replicate Tests

7.1 Model Terms, Estimated Coefficients, Coefficient Standard Deviations, Coefficient pValues, and Model Summary Statistics for the Gibbsite Concentration Models, Corresponding to the 43-inch and 120-inch Tanks, of the Form in Equation Obtained by Stepwise Regression.

7.2 Model Terms, Estimated Coefficients, Coefficient Standard Deviations, Coefficient pValues, and Model Summary Statistics for the Stainless Steel Concentration Models, Corresponding to the 43-inch and 120-inch Tanks, of the Form in Equation Obtained by Stepwise Regression.

7.3 Model Terms, Estimated Coefficients, Coefficient Standard Deviations, Coefficient pValues, and Model Summary Statistics for the Sand Concentration Models, Corresponding 
to the 43-inch and 120-inch Tanks, of the Form in Equation Obtained by Stepwise Regression

7.4 Model Terms, Estimated Coefficients, Coefficient Standard Deviations, Coefficient pValues, and Model Summary Statistics for the $\mathrm{ZrO}_{2}$ Concentration Models, Corresponding to the 43-inch and 120-inch Tanks, of the Form in Equation Obtained by Stepwise Regression

7.5 Model Terms, Estimated Coefficients, Coefficient Standard Deviations, Coefficient pValues, and Model Summary Statistics for the Gibbsite Concentration Models, Corresponding to the 43-inch and 120-inch Tanks, of the Form in Equation Obtained by Stepwise Regression.

7.6 Model Terms, Estimated Coefficients, Coefficient Standard Deviations, Coefficient pValues, and Model Summary Statistics for the Stainless Steel Concentration Models, Corresponding to the 43-inch and 120-inch Tanks, of the Form in Equation Obtained by Stepwise Regression.

7.7 Model Terms, Estimated Coefficients, Coefficient Standard Deviations, Coefficient pValues, and Model Summary Statistics for the Sand Concentration Models, Corresponding to the 43-inch and 120-inch Tanks, of the Form in Equation Obtained by Stepwise Regression

7.8 Model Terms, Estimated Coefficients, Coefficient Standard Deviations, Coefficient pValues, and Model Summary Statistics for the $\mathrm{ZrO}_{2}$ Concentration Models, Corresponding to the 43-inch and 120-inch Tanks, of the Form in Equation Obtained by Stepwise Regression

7.9 Model Terms, $\mathrm{R}^{2}$ Values, and Model Lack-of-Fit Assessment for Stepwise Regression Fits of Natural Logarithm of Component Concentrations in Models 5.5 and 5.8, Separately for Each of the Two Scaled Tanks.

7.10 Target $S V$, Density, and Resulting $K V$ Values Used in the Experimental Design and Prediction Profile Plots

8.1 Summary of Scaling Exponent Ranges for SS and Sand Concentrations Based on the Models in Section 7.1 and Section 7.2 


\subsection{Introduction}

The approach proposed by Washington River Protection Solutions (WRPS) to relate mixing and transfer performance (MTP) in scaled tanks to a full-scale, double shell tank (DST) consists of the following steps.

1. Using the Small Scale Mixing Demonstration (SSMD) platform, perform tests in two scaled tanks (43- and 120-inch diameters) ${ }^{(a)}$ to investigate the effects on MTP of several test parameters, including mixer-jet nozzle velocity.

2. Using the test data, develop mathematical models that relate the MTP for each of the two scaled tanks to the parameters varied in SSMD testing.

3. Hypothesize a scaling equation for MTP that relates mixer-jet nozzle velocity to tank diameter.

4a. If possible, combine the scaling equation from Step 3 with the mathematical models for the two scaled tanks from Step 2 to develop a single model that predicts MTP as a function of a scaling factor, mixer-jet nozzle velocity, and the other parameters varied in SSMD testing. The scaling factor could depend on the values of the test parameters.

4b. If Step 4a is not possible, utilize the models from Step 2 to calculate, for a grid of $K$ combinations of the test parameters from Step 1, the jet velocities in the 120-inch tank that give the same MTP values as a sequence of appropriate jet velocities in the 43-inch tank. This is referred to as equivalent performance (EP). Then, the EP pairs $\left[\left(43, U_{43, k}\right)\right.$ and $\left(120, U_{120, k}\right)$, $k=1,2, \ldots, K]$ can be substituted into the scaling equation of Step 3 to calculate the scaling factor for each EP pair in the sequence, for each grid point of combinations of the other test parameters. Hence, the scaling factor may depend on the mixer-jet nozzle velocity as well as the values of other test parameters.

5. Use the results from Steps 2-4 along with subject-matter theory, knowledge, computer models, and other sources to develop a methodology for quantifying MTP and/or scaling factor(s) appropriate for full scale.

This document addresses Steps 1 through 4, the results of which are limited to quantifying MTP and scaling factor(s) for the 43 -inch tank (1:21 scale) and 120-inch tank (1:8 scale). Step 5 is a separate work scope that will be performed and documented separately.

Section 2 discusses the SSMD scaling performance tests, including the parameters varied in the testing, the experimental region over which the parameters were varied, the original 22-test experimental design performed in the two scaled tanks, and the additional four test combinations performed in the two scaled tanks. Section 3 discusses the collection, preparation, and chemical analyses of samples. Section 3 also presents and graphically assesses the resulting concentration $(\mathrm{lb} / \mathrm{gal})$ data of the four simulant components: gibbsite $\left[\mathrm{Al}(\mathrm{OH})_{3}\right]$, stainless steel $(\mathrm{SS})$, sand, and zirconium dioxide $\left(\mathrm{ZrO}_{2}\right)$. Section 4 discusses the options considered for measures of MTP. Section 5 discusses (i) the model forms considered for relating component concentrations to test parameters, (ii) the statistical methods used to fit

(a) The diameter of the small tank is approximately 43.2 inches, but for convenience it is commonly referred to as 43 inches, a practice that is used in this report. 
the models and evaluate how well they fit the data, and (iii) the graphical method used to assess the sensitivity of component concentrations to variations in the test parameters. Section 6 presents a simple geometric scaling equation with a scaling exponent and discusses the EP approach used to calculate scaling exponents as functions of the test parameters. Section 7 presents and discusses the results of fitting the component concentration models from Section 5 to test data. Section 8 presents and discusses the results from applying the EP method of Section 6 to the models of Section 7 to obtain calculated scaling exponents as functions of test parameters that affect them. Section 9 lists the references cited in the document. Appendix A lists the parameter values and the component concentrations for pre-transfer samples and samples from five batch transfers for each of the 52 tests performed. Appendix B presents plots of component concentrations from pre-transfer samples and samples from five batch transfers for the 26 test combinations performed at the two scales. Appendix C presents plots of standard deviations (SDs) and percent relative standard deviations (\%RSDs) of component concentrations estimated from replicate tests. Appendix D presents prediction profile plots for component concentrations of gibbsite and $\mathrm{ZrO}_{2}$ (the plots for stainless steel and sand are presented in Section 7.4). 


\subsection{Small Scale Mixing Demonstration Scaling Performance Tests}

Two sets of scaling performance tests were conducted using the SSMD test platform. The first set consisted of 22 test combinations performed using each of the two scaled tanks, for a total of 44 tests. The 22 test combinations were based on a statistical experimental design constructed to investigate the effects of four test parameters. The second set of tests consisted of 4 test combinations of the test parameters that were not tested in the original set of 22 test combinations. The 4 additional combinations were selected by WRPS as being more likely to correspond to conditions expected during actual operations. These 4 additional test combinations were performed using each of the two scaled tanks, for a total of 8 tests. Hence, a total of $44+8=52$ tests were performed.

The test parameters and their possible settings are summarized in Section 2.1. The experimental design for the set of 22 test combinations performed at each scale is summarized in Section 2.2. The set of 4 additional test combinations performed at each scale is discussed further in Section 2.3.

\subsection{Experimental Parameters and Possible Settings}

Table 2.1 lists the parameters and their settings that were chosen for experimental investigation. Prior to testing, the mixer-jet nozzle velocity was expected to have the primary effect on MTP, but the base simulant, supernatant properties, and transfer-line capture velocity may also affect MTP. Supernatant viscosity (SV) was used to represent the combined effects of SV and supernatant density (Density) for the purposes of experimental design and modeling the test results. Lee et al. (2012a) stated that the relationship between Density and $S V$ is linear (Density $=1.259+0.0076 S V$ ) for the scaled performance SSMD tests. A technical reviewer of this report (Jim Fort, PNNL) pointed out that a more appropriate statement is that the linear relationship is between $\ln (S V)$ and Density. That relationship was given by Wells et al. (2011) in their Equation (3.3) with coefficients from their Table 3.4, which is applicable for liquid density $<1.41 \mathrm{~g} / \mathrm{mL}$. Wells et al. (2011) was the original reference for Figure 6.2 in Lee et al (2012b). Using the equation and coefficients from Wells et al. (2011) and solving for Density yields the more appropriate equation given by Density $=0.9567+0.1709 \ln (S V)$.

Table 2.1. Test Parameters and Possible Settings for Scaled Performance Testing

\begin{tabular}{lccc}
\hline Parameter & Symbol & Units & Possible Settings \\
\hline Tank Diameter & $D$ & Inches & $43.2,120$ \\
Jet Nozzle Velocity & $U$ & Coded & $-1,-0.5,0,0.5,1$ \\
Base Simulant & $B S$ & Coded & Typical (-1), High (1) \\
Supernatant Viscosity & $S V$ & $\mathrm{cP}$ & $3.3,8.0,14.6$ \\
Capture Velocity & $C V$ & $\mathrm{ft} / \mathrm{sec}$ & $3.8,7.3,11.3^{(\mathrm{a})}$ \\
\hline
\end{tabular}

(a) Ultimately, $C V$ was assumed to have a linear effect on MTP for the purposes of generating the experimental design. Three possible settings of $C V$ would not be needed with that assumption, but were specified because of a constraint that prohibits some combinations of $S V$ and $C V$ (discussed subsequently). 
The settings of $S V$ and $C V$ in Table 2.1 are not equally spaced, as would typically be chosen to provide for optimal coverage of the test space. The values were selected for reasons discussed by Lee (2012). The ranges of mixer-jet nozzle velocity were chosen so that -1 corresponds to the minimum velocity that can attain a $75 \%$ effective clearing radius with high base simulant. Note that this velocity is dependent on tank size. The mixer-jet nozzle velocity of +1 corresponds to the minimum velocity required to achieve a $100 \%$ effective clearing radius in the tank with typical base simulant. The remaining mixer-jet nozzle velocities were chosen to be evenly spaced between these minimum and maximum values. The compositions of the typical and high base simulants are listed in Table 2.2.

Table 2.2. Particulate Characteristics and Compositions of Typical and High Base Simulants

\begin{tabular}{lcccc}
\hline Component & $\begin{array}{c}\text { Solid Density } \\
\text { (g/mL) }\end{array}$ & $\begin{array}{c}\text { Median Particle } \\
\text { Size (micron) }\end{array}$ & $\begin{array}{c}\text { Typical Base } \\
\text { Simulant } \\
\text { (mass fraction) }\end{array}$ & $\begin{array}{c}\text { High Base } \\
\text { Simulant } \\
\text { (mass fraction) }\end{array}$ \\
\hline Small Gibbsite & 2.42 & 1.3 & 0.27 & 0 \\
Large Gibbsite & 2.42 & 10 & 0.44 & 0.03 \\
Small Sand & 2.65 & 57 & 0 & 0.35 \\
Medium Sand & 2.65 & 148 & 0.13 & 0 \\
Large Sand & 2.65 & 382 & 0 & 0.21 \\
Zirconium Oxide & 5.7 & 6 & 0.10 & 0.08 \\
Stainless Steel & 8.0 & 112 & 0.06 & 0.33 \\
\hline \multicolumn{5}{r}{} \\
\hline
\end{tabular}

A condition for the testing was that $C V$ must not be in the laminar flow regime. This condition resulted in excluding a subregion of $(S V, C V)$ combinations that would otherwise have been allowed per Table 2.1. A formula based on the Reynolds number for the least turbulent flow condition (transfer pump rate of $2.17 \mathrm{gpm}$ in the smaller tank) was used to exclude Newtonian test combinations that would have laminar flow:

$$
4948.16 \sqrt{2.17 C V}\left(\frac{1.259+0.0076 S V}{S V}\right)<2200 .
$$

This formula uses the test conditions and properties of the fluid to calculate the Reynolds number (the left hand side), where values less than 2200 correspond to laminar flow. See Section 3.1.4 of Lee (2012) for more details on the construction of Equation (2.1). Note that Lee (2012) used 2300 instead of 2200 in Equation (2.1), but doing so excluded a combination of $(S V, C V)$ that was considered desirable for testing. ${ }^{\text {(a) }}$ Hence, Lee et al. (2012a) used 2200 instead of 2300 in Equation (2.1) to develop the experimental design. The solid line in Figure 2.1 (a revision of Figure 1 in Lee et al. 2012a) shows the subregion of the two-dimensional $(S V, C V)$ space allowed by Equation (2.1). The figure shows that with a higher $S V, C V$ must not be too low or else laminar flow would result.

(a) A supernatant with a density of $1.37 \mathrm{~g} / \mathrm{mL}$, a viscosity of $8 \mathrm{cP}$, and a capture velocity of $3.8 \mathrm{ft} / \mathrm{sec}$ is on the edge of the excluded region. 


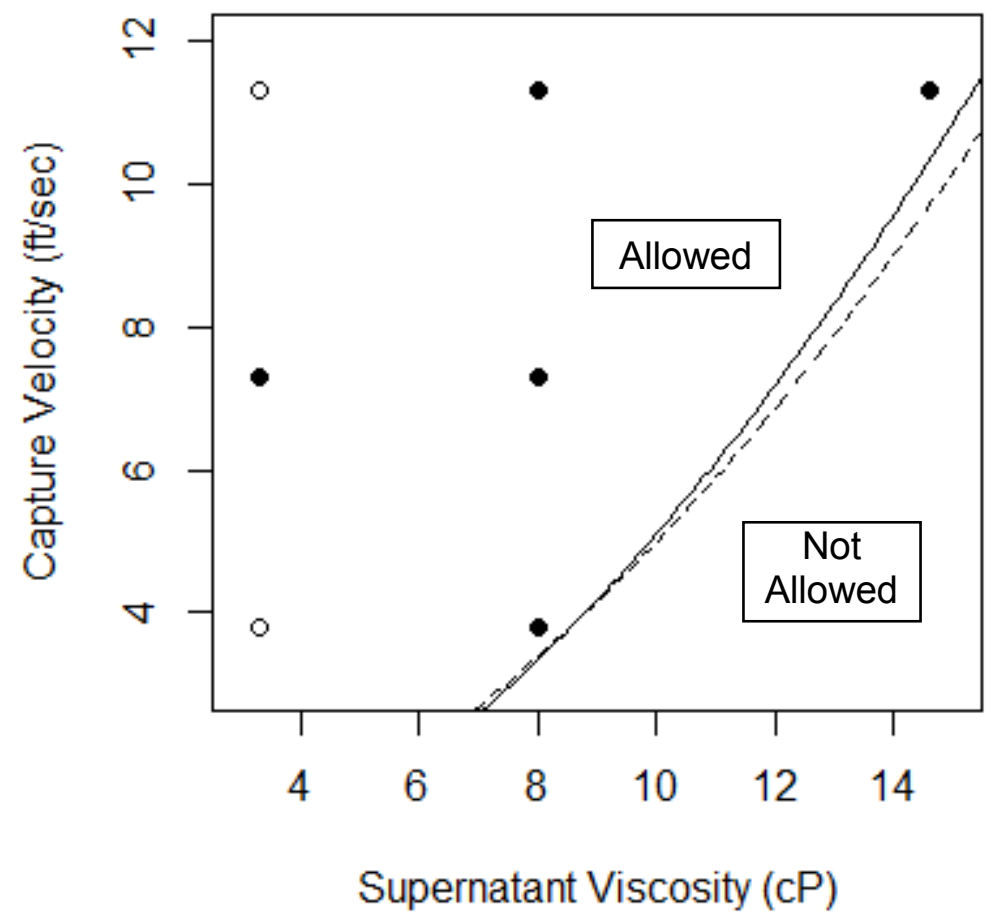

Figure 2.1. Subregion of Permissible Combinations of Supernatant Viscosity and Transfer-Line Capture Velocity. The $(S V, C V)$ combinations that were available to be selected for the experimental design are shown as open circles, while the combinations actually selected for the experimental design are shown as closed circles. (For Information Only)

As discussed previously, the relationship Density $=1.259+0.0076 \mathrm{SV}$ that appears in Equation (2.1) is not as appropriate as the equation given above. Substituting this equation into Equation (2.1) yields

$$
4948.16 \sqrt{2.17 C V}\left(\frac{0.9567+0.1709 \ln (S V)}{S V}\right)<2200 .
$$

This version of the constraint is shown in Figure 2.1 as a dashed line, which is not substantially different from the solid line based on Equation (2.1). Hence, there was little impact to selecting the parameter combinations for testing to using the less appropriate relationship between $S V$ and $C V$.

\subsection{Experimental Design of Tests at Both Scales}

Lee et al. (2012a) describe how the experimental design of 22 test combinations of $U, B S, S V$, and $C V$ was selected and performed using each of the two SSMD scaled tanks. The total of 44 tests was determined as the number of tests that could be performed, given the budget constraints. Of the 22 test combinations performed in each tank, 18 were distinct and 4 were allotted to replicates. Replicates provide for (i) quantifying the uncertainties associated with the test results and (ii) statistically assessing the lack-of-fit for models fitted to the resulting data. 
The 18 distinct test combinations were selected using a type of optimal experimental design (Atkinson and Donev 1992) referred to as Bayesian I-optimal experimental design (analogous to Bayesian D-optimal design discussed by DuMouchel and Jones 1994; JMP 2012) to support fitting the model

$$
\begin{aligned}
P M_{i j k} & =b_{0}^{i j k}+b_{1}^{i j k} U_{i}+b_{2}^{i j k} U_{i}^{2}+b_{3}^{i j k} U_{i}^{3}+b_{4}^{i j k} B S+b_{5}^{i j k} S V+b_{6}^{i j k} S V^{2}+b_{7}^{i j k} C V \\
& +b_{8}^{i j k}\left[B S * U_{i}\right]+b_{9}^{i j k}\left[B S * U_{i}^{2}\right]+b_{10}^{i j k}[B S * S V]+b_{11}^{i j k}\left[B S * S V^{2}\right] \\
& +b_{12}^{i j k}\left[S V * U_{i}\right]+b_{13}^{i j k}\left[S V^{2} * U_{i}\right]+b_{14}^{i j k}\left[S V * U_{i}^{2}\right]+b_{15}^{i j k}\left[S V^{2} * U_{i}^{2}\right]
\end{aligned}
$$

where $\quad P M_{i j k}=$ mixing and transfer performance metric $(P M)$ of the $j^{\text {th }}$ base-simulant component in the $k^{\text {th }}$ batch transfer $(k=1,2,3,4,5)$ from the $i^{\text {th }}$ tank $(i=1,2)$ (the units depend on the definition of the PM)

$U_{i}=$ mixer-jet nozzle velocity in the $i^{\text {th }} \operatorname{tank}(\mathrm{ft} / \mathrm{sec})$

$B S=$ base simulant (typical, high)

$S V=$ supernatant viscosity $(\mathrm{cP})$

$C V=$ capture velocity $(\mathrm{ft} / \mathrm{sec})$

$b_{0}^{i j k}$ to $b_{15}^{i j k}=$ model coefficients for the $j^{\text {th }}$ base-simulant component in the $k^{\text {th }}$ batch transfer from the $i^{\text {th }}$ tank.

The development of this model is discussed by Greer (2012) and Lee et al. (2012a). The Bayesian Ioptimal design approach selected tests to minimize the uncertainty in predictions with the model in Equation (2.3) averaged over the experimental space, while also protecting against higher-order terms possibly being needed in the model.

Table 2.3 lists the 22 test combinations, including the 4 replicate tests, in the final experimental design. This set of 22 test combinations involved exchanging one of the 18 distinct test combinations in the statistical experimental design with another test so that there would be one test combination $(B S=$ High, $S V=14.6, C V=11.3$ ) with four values of $U$ for each of the two scaled tanks (Tests 18-21 in Table 2.3). See Greer (2012) for the details of how the test to be replaced and the test to replace it with were selected.

Inspection of the experimental design in Table 2.3 identifies several interesting observations, some of which are important relative to modeling and analyzing the data resulting from testing.

1. For Tests $1-5$, when $S V=3.3 \mathrm{cP}$ (the smallest value), $C V=7.3 \mathrm{ft} / \mathrm{sec}$ (the middle value). For Tests $18-22$, when $S V=14.6 \mathrm{cP}$ (the largest value), $C V=11.3$ (the largest value). Only when $S V=8 \mathrm{cP}$ is $\mathrm{CV}$ allowed to vary over its full range $3.8-11.3 \mathrm{ft} / \mathrm{sec}$. Hence, the combinations of $(S V, C V)=(3.3,3.8)$ and $(3.3,11.3)$ do not appear in the experimental design in Table 2.3, even 
though they are permissible as shown in Figure 2.1. ${ }^{\text {(a) }}$ Having only five rather than the possible seven combinations of $(S V, C V)$ in the test matrix is less than ideal for being able to separately estimate the effects of $S V$ and $C V$ as well as the interactions involving $S V$ (recall that it was assumed that $C V$ has a linear effect only and does not interact with other test parameters).

3. The experimental design in Table 2.3 is highly "fractionated." That is, the total number of possible combinations of $U, B S, S V$, and $C V$ is $5 \times 2 \times 3 \times 3=90$, but only 18 distinct combinations are tested. This degree of fractionation is possible because the higher-order singleparameter and interactive effects of these four test parameters are assumed to be negligible compared to the effects of the terms in Equation (2.3). Usual statistical practice would be to select at least 10 more distinct test combinations relative to the number of coefficients in Equation (2.3), but resources for testing did not permit doing this, as discussed previously.

4. Although the model in Equation (2.3) specifies only a linear effect for $C V$ and no interactions involving $C V$, three levels of $C V$ are included in the design. This could be due to (i) the flowregime constraint in Equation (2.1) that incorporates $C V$, thus restricting the design space (as shown in Figure 2.1) and/or (ii) the Bayesian I-optimal approach to developing the experimental design.

5. Five settings of mixer-jet nozzle velocity were selected, although only four settings are necessary to fit a cubic polynomial in velocity. This could be the result of some of the interaction effects in the design model, the Bayesian I-optimal design approach, or the restriction on the design region imposed by the flow-regime constraint in Equation (2.1).

Ultimately, Items 1 and 2 are the most critical in terms of consequences when modeling mixing and transfer performance and developing scaling relationships.

Finally, the 22 test combinations were performed in the random run order shown in Table 2.3. Tests using the 43-inch and 120-inch tanks for a given one of the 22 test combinations were run during the same time period and were completed before the next of the 22 test combinations was tested. As discussed by Lee et al. (2012a), this is a restriction on randomization that requires a more complicated statistical analysis methodology if a single model applicable to both tanks is developed (Option 4a in Section 1). However, Option $4 \mathrm{~b}$ was taken, so it was not necessary to use the more complicated statistical methodology to fit the MTP models.

\footnotetext{
(a) After the testing was completed, it was discovered that these two combinations of $S V$ and $C V$ were not represented in the experimental design because of the specifics of how the Bayesian I-optimal design approach was implemented by Greer (2012). Although too late, it was possible to obtain experimental designs containing one or both of the $S V$ and $C V$ combinations.
} 
Table 2.3. Experimental Design for Each of Two Tank Scales in SSMD Testing

\begin{tabular}{|c|c|c|c|c|c|}
\hline $\begin{array}{l}\text { Test } \\
\text { Number }^{(a)}\end{array}$ & $\begin{array}{c}\text { Random } \\
\text { Run } \\
\text { Order } \\
\end{array}$ & $\begin{array}{c}\text { Jet Velocity, } \\
U \\
\text { (coded) }^{(\mathbf{b})}\end{array}$ & $\begin{array}{c}\text { Base } \\
\text { Simulant, } \\
B S \\
\end{array}$ & $\begin{array}{c}\text { Target } \\
\text { Supernatant } \\
\text { Viscosity, } S V \\
\text { (cP) }\end{array}$ & $\begin{array}{c}\text { Target } \\
\text { Capture } \\
\text { Velocity, } C V \\
\text { (ft } / \mathbf{s e c}) \\
\end{array}$ \\
\hline 1 & 8 & -1 & High & 3.3 & 7.3 \\
\hline 2 & 1 & 0 & High & 3.3 & 7.3 \\
\hline 3 & 14 & -0.5 & Typical & 3.3 & 7.3 \\
\hline 4 & 11 & 1 & Typical & 3.3 & 7.3 \\
\hline 5 (Rep. 4) & 20 & 1 & Typical & 3.3 & 7.3 \\
\hline 6 & 21 & -0.5 & High & 8 & 3.8 \\
\hline 7 (Rep. 6) & 3 & -0.5 & High & 8 & 3.8 \\
\hline 8 & 2 & 0.5 & High & 8 & 3.8 \\
\hline 9 & 13 & -1 & High & 8 & 7.3 \\
\hline 10 & 9 & 1 & High & 8 & 7.3 \\
\hline 11 & 12 & 0 & High & 8 & 11.3 \\
\hline 12 (Rep. 11) & 5 & 0 & High & 8 & 11.3 \\
\hline 13 & 22 & 0 & Typical & 8 & 3.8 \\
\hline 14 & 19 & -1 & Typical & 8 & 7.3 \\
\hline 15 & 7 & 1 & Typical & 8 & 7.3 \\
\hline 16 & 16 & 0 & Typical & 8 & 11.3 \\
\hline 17 (Rep. 16) & 4 & 0 & Typical & 8 & 11.3 \\
\hline 18 & 10 & -1 & High & 14.6 & 11.3 \\
\hline 19 & 15 & 0 & High & 14.6 & 11.3 \\
\hline 20 & 17 & 1 & High & 14.6 & 11.3 \\
\hline 21 & 18 & -0.5 & High & 14.6 & 11.3 \\
\hline 22 & 6 & 0.5 & Typical & 14.6 & 11.3 \\
\hline
\end{tabular}

(a) Replicate test numbers are shown in parentheses.

(b) Because the range of mixer-jet nozzle velocity settings was to be different for the two tanks because of geometric scaling, coded values were used to develop the experimental design. A -1 coded value represents the lower limit for a given scale, while $\mathrm{a}+1$ coded value represents the upper limit for that scale. The $-0.5,0$, and 0.5 coded values represent actual values that can be calculated by linear interpolation.

\subsection{Four Additional Test Combinations}

After the 44 tests ( 22 at each of the two scales) were completed, an additional 8 tests ( 4 at each of the two scales) were performed. The additional tests had been planned into the original budget, but the test combinations were not selected until after the original 44 tests were completed. Also, the additional tests were selected early in the data analysis process, so the results of data analyses were not factored into choosing the additional tests.

Because of the highly fractionated nature of the 18 distinct combinations in Table 2.3 (see Item 2 of Section 2.2), one option for selecting the additional 4 test combinations was a statistical optimal design approach to augment the 22-test experimental design with the 4 additional test combinations that would provide the best improvement in support for fitting the model in Equation (2.3). Within this approach, 
options that yielded the additional 4 test combinations containing one or both of the "missing" $(S V, C V)$ combinations discussed in Item 2.1 were considered. However, the additional 4 test combinations selected by this approach included extreme (i.e., vertex) points of the test parameter space that had not been tested previously. Ultimately, WRPS decided that it would be better to select the additional 4 test combinations to include parameter combinations that were more realistic in terms of actual operations.

The four additional test combinations (performed in both scaled tanks) are listed in Table 2.4. Note that all four test combinations are at the $(S V=3.3, C V=3.8)$ combination, which is one of the two $(S V, C V)$ combinations that was not included in the experimental design of Table 2.3. The first three of the four test combinations were chosen to be $U$ variations typical of the anticipated waste feed delivery conditions. These variations included $B S=$ Typical, the typical density-typical viscosity supernatant $(S V=3.3 \mathrm{cP})$, and a $C V=3.8 \mathrm{ft} / \mathrm{s}$. This $C V$ value was selected based on preliminary slurry pump design information that included a 3.9-inch-diameter inlet for the 140-gpm flow. The mixer-jet nozzle velocities were selected from the middle of the range of values (coded values of $-0.5,0$, and 0.5 ). The fourth additional test combination was a variation in the base simulant from one of the three new tests. The test combination was with the high base simulant, the typical density-typical viscosity supernatant, the middle mixer-jet nozzle velocity (coded value of 0 ), and $C V=3.8 \mathrm{ft} / \mathrm{s}$.

Table 2.4. Additional Four Test Combinations for Each of Two Tank Scales in SSMD Testing

\begin{tabular}{|c|c|c|c|c|c|c|}
\hline $\begin{array}{l}\text { Test } \\
\text { Number }\end{array}$ & $\begin{array}{c}\text { 43-inch } \\
\text { Tank } \\
\text { Run Order }\end{array}$ & $\begin{array}{c}\text { 120-inch Tank } \\
\text { Run Order }\end{array}$ & $\begin{array}{c}\text { Jet } \\
\text { Velocity, } U \\
\text { (coded) }^{(\mathbf{a})}\end{array}$ & $\begin{array}{c}\text { Base } \\
\text { Simulant, } B S\end{array}$ & $\begin{array}{c}\text { Target } \\
\text { Supernatant } \\
\text { Viscosity, } S V \\
\text { (cP) }\end{array}$ & $\begin{array}{c}\text { Target } \\
\text { Capture } \\
\text { Velocity, } C V \\
\text { (ft/sec) }\end{array}$ \\
\hline 23 & 23 & 24 & -0.5 & Typical & 3.3 & 3.8 \\
\hline 24 & 24 & 23 & 0 & Typical & 3.3 & 3.8 \\
\hline 25 & 25 & 25 & 0.5 & Typical & 3.3 & 3.8 \\
\hline 26 & 26 & 26 & 0 & High & 3.3 & 3.8 \\
\hline
\end{tabular}

(a) The range of mixer-jet nozzle velocity settings was to be different for the two tanks because of geometric scaling, so coded values were used to specify the test values. The velocity coding used for the original 22 test combinations in each scaled tank was also used for the additional 4 test combinations in each scaled tank.

Table 2.5 contains the target mixer-jet pump velocities (in $\mathrm{ft} / \mathrm{s}$ ) that correspond to the coded jet velocity, $U$, in Tables 2.3 and 2.4. As mentioned in Tables 2.3 and 2.4, the actual mixer-jet nozzle velocity settings were different for the two tank sizes because they were based on geometric scaling.

Table 2.5. Target Mixer-Jet Pump Velocities (ft/s)

\begin{tabular}{ccc}
\hline $\begin{array}{c}\text { Jet Velocity, } \boldsymbol{U} \\
\text { (coded) }\end{array}$ & $\begin{array}{c}\text { 43-inch Tank } \\
\text { Target Jet Velocity (ft/s) }\end{array}$ & $\begin{array}{c}\text { 120-inch Tank Target Jet } \\
\text { Velocity (ft/s) }\end{array}$ \\
\hline-1 & 18.24 & 28.72 \\
-0.5 & 22.14 & 31.12 \\
0 & 26.05 & 33.51 \\
0.5 & 29.96 & 35.90 \\
1 & 33.87 & 38.30 \\
\hline
\end{tabular}




\subsection{Experimental Data}

During the SSMD system performance testing (Lee 2012), the contents of a tank (43-inch or 120-inch) were mixed and transferred in five batches at the conditions prescribed in Table 2.3 and Table 2.4. The collection, preparation, and analysis of samples leading to the experimental data analyzed in this report are discussed in Section 3.1. The resulting data for the 22 test combinations plus 4 additional test combinations for each of the two scaled tanks (52 tests total) are presented and discussed in Section 3.2. Section 3.3 discusses graphical assessments of the data.

\subsection{Collection, Preparation, and Chemical Analysis of Samples}

Sections 3.1.1 through 3.1.3 discuss the collection of initial samples, preparation of those samples including subsampling, and laboratory chemical analyses of the subsamples, respectively. Section 3.1.4 discusses normalizing the analyzed weight percent ( $\mathrm{wt} \%$ ) solids values of the four solids components of interest: gibbsite, SS, sand, and $\mathrm{ZrO}_{2}$. Section 3.1.5 discusses the collection of other data on the test conditions. These discussions cover pre-transfer samples and samples from each of the five batch transfers for tests performed using each of the two scaled tanks.

\subsubsection{Collection of Initial Samples}

The initial pre-transfer and batch-transfer samples were collected for each test in the 43-inch and 120inch tanks as follows.

Pre-Transfer Initial Samples: Two pre-transfer samples were taken (into 5-gal buckets) from the transfer loop operating in a recirculation mode. Each pre-transfer sample corresponded to one or two complete revolutions of the mixer-pump jets in a tank. One sample was sent to the laboratory for analysis; the other was archived.

Batch-Transfer Initial Samples: For the 43-inch tank, the entire batch transfer was collected in a mixer barrel as the initial sample ( $\sim 16$ gal). For the 120-inch tank, a diversion sample was taken every 19 th or 20th revolution of the mixer-pump jets, which corresponds to four or five times (depending on the rotation speed) during a batch transfer. Each such diversion sample was approximately 3 to 4 gal. All four or five diversions from a batch transfer were collected in the same mixer barrel, yielding a composite sample of about $16.1 \mathrm{gal}$.

\subsubsection{Preparation of Samples for Chemical Analyses}

The preparation of initial samples and subsampling for chemical analyses was the same for tests conducted in the 43 -inch and 120 -inch tanks, as discussed in the following.

Pre-Transfer Samples: The initial pre-transfer samples were decanted by letting them sit in a 5-gal bucket until the solids settled and a clear liquid (the supernatant) rose to the top. The clear liquid was removed by a pump. After the supernatant was removed, the wet solids were transferred into $1000-\mathrm{mL}$ bottles for shipment to the analytical laboratory. Typically only one $1000-\mathrm{mL}$ bottle was needed for the pre-transfer wet solids. 
Batch-Transfer Samples: The initial batch-transfer samples were decanted by letting them sit in mixer barrels until the solids settled and a clear liquid (the supernatant) rose to the top. Then the clear supernatant was removed by a pump. If solids were removed with the supernatant, the removed slurry was mixed thoroughly and a sample was collected in a 1000-mL bottle. An additional sample was taken and archived. The sample was sent to the analytical laboratory for chemical analysis so the amount of solids lost during this part of the procedure could be calculated subsequently. The weight of the sample in the barrel before decanting and the weight of the supernatant removed were taken.

The mixer barrel containing the remaining slurry (after removal of clear supernatant) was then rotated on a "cement-mixer" device until it appeared to be well mixed with a "pancake-batter" consistency. If the slurry did not have the right consistency (i.e., too runny), it was left to sit on the mixer device until more supernatant could be decanted. When the correct consistency was achieved, four separate samples were taken by scoop from four quadrants of the mixer barrel, and each scoop was put into a pre-weighed 250$\mathrm{mL}$ container. An additional four $250-\mathrm{mL}$ samples were taken (one from each quadrant) and were archived in case they were needed for later analysis. The final weight of each of the four $250-\mathrm{mL}$ containers (with samples) was recorded. These $250-\mathrm{mL}$ containers with samples were sent to an analytical laboratory for chemical analysis.

\subsubsection{Laboratory Chemical Analyses}

Initially, the analytical laboratory was sent two kinds of samples to analyze (i) samples of wet solids that remained after decanting the supernatant and (ii) samples of supernatants containing solids. Initially, it was assumed that these supernatant samples contained only gibbsite, so they were analyzed for only total $\mathrm{wt} \%$ solids. Based on this assumption, the recovery for one of the samples came back as $180 \%$ of the total amount of added gibbsite, which is impossible. A subset of the retained solids from the supernatant samples was then sent for further chemical analysis to determine the mass fractions of the components in the solids from the supernatants. The results of these tests indicated that gibbsite, sand, and $\mathrm{ZrO}_{2}$ were sometimes present in the supernatant solutions. Hence, the remaining solids retained from the supernatant samples were sent for chemical analyses. In summary, although supernatant samples were originally sent to the analytical laboratory for total wt\% solids analyses only, later retained solids from the supernatant samples were sent to the analytical laboratory to determine the mass fractions of components in the solids from the supernatants.

Wet Solids Samples: At the analytical laboratory, the contents of 1000-mL bottles (pre-transfer sample), or of a $250-\mathrm{mL}$ container (batch transfer sample), were poured into a wax-bottom beaker (which allows taking core samples to the bottom of the settled material). Four or five core samples (depending on the volume of solids captured per core) were taken from a beaker and combined. The combined core samples were mixed, filtered, dried, coned, and quartered to achieve smaller subsamples compared to the original samples submitted to the laboratory. The smaller sample was then washed and dried, and then small enough subsamples for the analytical tests were taken and destructively tested. The analytical methods for the components involved lithium borate fusion (gibbsite, sand, and $\mathrm{ZrO}_{2}$ ), microwave digestion ( $\mathrm{SS}$ and $\mathrm{ZrO}_{2}$ ), and aqua regia digestion (SS), with chemical analysis via inductively coupled plasma-atomic emission spectroscopy.

Supernatant Samples: The laboratory decanted the supernatant samples and then further processed the wet solids as follows. The wet solids were filtered, rinsed, and dried, and then the dry solids were 
weighed. The solids content of the supernatant was expressed as a mass fraction by taking the ratio of the weight of the dried solids to the weight of the entire original supernatant sample (solids and liquid).

Subsequently, using the previously separated solids from the supernatant samples, the laboratory used the same preparation steps and then sampled the dried solids. The solids samples were digested and analyzed by $\mathrm{x}$-ray fluorescence to determine the mass fraction of each component (gibbsite, $\mathrm{SS}$, sand, $\mathrm{ZrO}_{2}$ ) in the sample.

Results: The main results from the analytical laboratory were the total $\mathrm{wt} \%$ solids and analyzed wt $\%$ solids of each of the base-simulant components (gibbsite, SS, sand, and $\mathrm{ZrO}_{2}$ ) in each sample relative to the total solids. This included slurry samples after decanting the supernatant, as well as samples of supernatants that contained solids. Ultimately, for each test there were at least 1 (pre-transfer) +4 (batch subsamples) $\times 5$ (batch transfers) $=21$ sets of component wt $\%$ results. For supernatant samples that contained solids, there were also corresponding sets of results.

Acceptance Criterion: The analytical laboratory had an acceptance criterion that required the sum of the four component $\mathrm{wt} \%$ values to be within 90 to $110 \mathrm{wt} \%$; otherwise, the sample analysis was repeated. Investigation of the data showed that in a few cases, this must not have been done, because the sum of the component $\mathrm{wt} \%$ solids was outside the specified range.

\subsubsection{Normalization of Analyzed Component Weight Percent Values}

Ideally, a certified, representative standard ${ }^{(a)}$ would have been analyzed along with each group of SSMD test samples. This would have provided for assessing analyzed values for bias and correcting for bias if detected. However, it is difficult to produce and maintain certified, representative slurry standards and then sample them for analysis, especially over a range of liquid and solids conditions. Hence, no standards were analyzed along with test samples.

An indirect way for assessing whether analyzed $\mathrm{wt} \%$ values of the components may be biased is to assess whether the distribution of total analyzed $\mathrm{wt} \%$ values is symmetric around $100 \mathrm{wt} \%$, with the mean not statistically significantly different from $100 \mathrm{wt} \%$. If so, that indicates the analytical procedure did not consistently underestimate or overestimate the $\mathrm{wt} \%$ values of the components.

Over the analyses of pre-transfer and transfer-batch samples from the 44 tests (the 22 test combinations in Table 2.3 with each of the two scaled tanks), the mean total wt $\%$ value was not statistically different from $100 \mathrm{wt} \%$. The mean total $\mathrm{wt} \%$ from the eight additional tests (the 4 test combinations in Table 2.4 with each of the two scaled tanks) was $97.5 \mathrm{wt} \%$, which was statistically different from $100 \mathrm{wt} \%$. There was no basis (i.e., representative standards analyzed with test samples) to assess whether there was preferential bias in one or more of the four components (gibbsite, SS, sand, and $\mathrm{ZrO}_{2}$ ). Hence, the analyzed component wt\% solids values for each sample (pre-transfer and each batch transfer) were "normalized" to mass fractions totaling 1.0 using the following simple formula:

(a) Here, "representative" means that the standard has a composition within the region of compositions being analyzed, while "certified" means that a sufficient number of high-quality analyses have been completed to determine the mean composition of the components with relatively small uncertainties. 


$$
x_{j}=\frac{w_{j}}{\sum_{j=1}^{q} w_{j}}
$$

where $w_{j}$ denotes the analyzed wt $\%$ value of the $j^{\text {th }}$ base-simulant component, $q=4$ is the number of basesimulant components (gibbsite, $\mathrm{SS}$, sand, and $\mathrm{ZrO}_{2}$ ), and $x_{j}$ is the normalized mass fraction of the $j$ th basesimulant component such, that $0 \leq x_{j} \leq 1$ and $\sum_{j=1}^{q} x_{j}=1$.

There are more complicated normalization formulas that take into account information about the uncertainties in each of the analyzed component wt\% values (Weier and Piepel 2003), but it was not clear that all of the needed information was available to apply these formulas. Weier and Piepel (2003) note that normalized component $\mathrm{wt} \%$ values have smaller uncertainties than non-normalized ones, provided that analyzed values are not biased or have been bias-corrected. Hence, it is advantageous to normalize the analyzed component $\mathrm{wt} \%$ values.

\subsubsection{Collection of Other Data}

For each test combination and tank size (hereafter called a "test"), the following information was collected: (i) the average calculated values of mixer-jet nozzle velocity, ${ }^{\text {(a) }}$ (ii) the actual supernatant viscosity and actual density before base particulates were added, and (iii) the average calculated values of transfer-line capture velocity ${ }^{(b)}$ over the period of the test.

\subsection{Experimental Data for the 26 Test Combinations}

The experimental data for the 22 test combinations performed at both scales (44 tests) in Table 2.3 and the 4 additional test combinations performed at both scales ( 8 tests) in Table 2.4 are presented in Appendix A. Table A.1 lists, for each test, the planned (target) values as well as actual values (when available) of the test parameters $(U, B S, S V, C V)$, along with other relevant information about the test conditions (e.g., the actual kinematic viscosity, which is used subsequently in modeling the experimental results). Table A. 2 of Appendix A lists for the 52 tests the concentrations (lb/gal slurry) of the four solids components (gibbsite, SS, sand, and $\mathrm{ZrO}_{2}$ ) for the pre-transfer samples and the five transfer batches.

\footnotetext{
${ }^{(a)}$ Mixer-jet nozzle velocity was not measured. Rather, the mixer-jet flow rate $\left(Q_{M J}\right)$ was monitored by a flow meter that reports gallons per minute. The nozzle velocity $\left(U_{N}\right)$ was then calculated by dividing $Q_{M J}$ by the nozzle area $\left(A_{N}\right)$, namely $U_{N}=Q_{M J} / A_{N}$.

(b) Transfer-line capture velocity was not be measured. Rather, similar to the mixer-jet nozzle velocity, the transferpump flow rate $\left(Q_{T P}\right)$ was monitored by a flow meter that reports gallons per minute. The transfer-line capture velocity $(\mathrm{CV})$ was calculated by dividing $Q_{T P}$ by the inlet area for the transfer pump suction $\left(A_{T P}\right)$, namely $C V=$ $Q_{T P} / A_{T P}$.
} 
The formula used to calculate a component concentration is

$$
C_{j}^{k}=\frac{\left(f_{j, D S, W S}^{k}\right)\left(f_{D S, W S}^{k}\right)\left(M_{W S}^{k}\right)+\left(f_{j, D S, D}^{k}\right)\left(f_{D S, D}^{k}\right) M_{D}^{k}}{V_{k}}
$$

where $\quad C_{j}^{k}=$ concentration (lb/gal slurry) of the $j^{\text {th }}$ component in the $k^{\text {th }}$ batch

$j=$ component $j$ (gibbsite, $\mathrm{SS}$, sand, or $\mathrm{ZrO}_{2}$ )

$k=$ batch $k(k=0$ for pre-transfer, $k=1-5$ for batch transfers 1 to 5$)$

$D S=$ dry solids

$W S=$ wet solids (what remains of the sample after decanting the liquid)

$D=$ decant solution (solution that sits at the top of the sample after settling of solids, may contain solids)

$f_{j, D S, W S}^{k}=$ normalized mass fraction (see Section 3.1.4) of the $j^{\text {th }}$ component in the dry solids remaining after drying the wet solids from the $k^{\text {th }}$ batch sample. For transfer batches $1-5$, this quantity is obtained by averaging the normalized mass fractions of a component in the four quadrant samples. For pre-transfer, there is only one sample, so no averaging is involved.

$f_{D S, W S}^{k}=$ mass fraction of the wet solids sample that is dry solids from the $k^{\text {th }}$ batch sample.

For batches 1-5, this quantity is obtained by averaging the mass fractions over the four quadrant samples. For pre-transfer there is only one sample, so no averaging is involved.

$M_{W S}^{k}=$ mass of wet solids in the slurry sample from the $k^{\text {th }}$ batch after decanting (lb)

$f_{j, D S, D}^{k}=$ normalized mass fraction (see Section 3.1.4) of the $j^{\text {th }}$ component in the dry solids remaining after filtering, rinsing, and drying the decant solution from the $k^{\text {th }}$ batch sample

$f_{D S, D}^{k}=$ mass fraction of the decant solution that is dry solids from the $k^{\text {th }}$ batch sample

$M_{D}^{k}=$ mass of wet solids in the decant solution from the $k^{\text {th }}$ batch after further decanting the liquids from the sample (lb)

$V_{k}=$ calculated volume of the $k^{\text {th }}$ batch sample before decanting (gal slurry). The flow rate (as calculated by taking the ratio of the mass flow and specific gravity measured by the Coriolis meter) was integrated over a specified time interval to calculate the totalized flow volume for the $k^{\text {th }}$ batch sample before decanting.

In calculating $f_{j, D S, W S}^{k}$ and $f_{D S, W S}^{k}$ for the batch transfer samples, it was assumed that the masses for the four quadrant samples were approximately equal and that any differences due to unequal sample masses were negligible. Ultimately, the thing to notice about Equation (3.2) is that the component concentrations 
account for the masses of components in the settled samples as well as the masses of components contained in supernatant (referred to as decant solution above).

Table 3.1 lists the minimums and maximums of the planned and actual values of $U, S V$, and $C V$, as well as actual values of kinematic viscosity (which is used instead of $S V$ as discussed subsequently in the report). Table 3.2 lists the minimums and maximums of the averaged component concentrations for each of the two tank scales. The minimums and maximums in Table 3.1 and Table 3.2 are over all 26 test combinations ( 22 original plus 4 additional) for each of the two scaled tanks.

Table 3.1. Minimum and Maximum Planned and Actual Values of Mixer-Jet Nozzle Velocity (U), Supernatant Viscosity (SV), Capture Velocity (CV), and Actual Values of Kinematic Viscosity (KV)

\begin{tabular}{c|cl|cc|cc}
\hline \multicolumn{2}{c|}{} & & & \multicolumn{2}{c|}{ Planned } & \multicolumn{2}{c}{ Actual } \\
\hline $\begin{array}{c}\text { Tank Diameter } \\
\text { (inches) }\end{array}$ & Parameter & Units & Minimum & Maximum & Minimum & Maximum \\
\hline \multirow{4}{*}{43} & $U$ & $\mathrm{ft} / \mathrm{sec}$ & 18.24 & 33.87 & 18.14 & 36.04 \\
& $S V$ & $\mathrm{cP}$ & 3.3 & 14.6 & 2.2 & 15.4 \\
& $C V$ & $\mathrm{ft} / \mathrm{sec}$ & 3.8 & 11.3 & 3.78 & 11.26 \\
& $K V$ & $\mathrm{cSt}$ & 2.57 & 10.66 & 1.75 & 11.41 \\
\hline \multirow{4}{*}{120} & $U$ & $\mathrm{ft} / \mathrm{sec}$ & 28.72 & 38.30 & 28.59 & 41.17 \\
& $S V$ & $\mathrm{cP}$ & 3.3 & 14.6 & 2.3 & 17.1 \\
& $C V$ & $\mathrm{ft} / \mathrm{sec}$ & 3.8 & 11.3 & 3.70 & 11.20 \\
& $K V$ & $\mathrm{cSt}$ & 2.57 & 10.66 & 1.78 & 12.62 \\
\hline
\end{tabular}

Table 3.2. Minimum and Maximum Component Concentration (lb/gal) Values Over the Pre-Transfer and Five Batch Transfer Values for Each Test and Over the 26 Test Combinations for Each Scaled Tank Separated by Base Simulant $(B S)$ type

\begin{tabular}{c|c|cc|cc}
\hline \multirow{2}{*}{$\begin{array}{c}\text { Tank Diameter } \\
\text { (inches) }\end{array}$} & Component & \multicolumn{2}{|c|}{$\begin{array}{c}\text { Component Concentration } \\
\text { (lb/gal) for } \boldsymbol{B S} \text { = Typical }\end{array}$} & \multicolumn{2}{c}{$\begin{array}{c}\text { Component Concentration } \\
\text { (lb/gal) for } \boldsymbol{B S} \text { = High }\end{array}$} \\
\cline { 3 - 6 } & Gibbsite & 0.7365 & 1.3334 & 0.0273 & 0.0574 \\
43 & Stainless Steel & 0.00053 & 0.0643 & 0.0040 & 0.4294 \\
& Sand & 0.0947 & 0.2422 & 0.3598 & 0.9929 \\
& $\mathrm{ZrO}_{2}$ & 0.1005 & 0.1703 & 0.0717 & 0.1324 \\
\hline \multirow{4}{*}{120} & Gibbsite & 0.6947 & 1.3570 & 0.0221 & 0.0596 \\
& Stainless Steel & 0.0103 & 0.0923 & 0.0477 & 0.5397 \\
& Sand & 0.1365 & 0.2825 & 0.4204 & 1.1586 \\
& $\mathrm{ZrO}_{2}$ & 0.0999 & 0.1811 & 0.0535 & 0.1493 \\
\hline
\end{tabular}




\subsection{Graphical Assessment of Component Concentrations Data}

Section 3.3.1 presents the results of graphical displays of component concentration data plotted versus "batches" (pre-transfer and the five transfer batches) for each of the 26 test combinations performed at each tank scale. Section 3.3.2 presents the results of plotting component concentrations versus mixer-jet nozzle velocity $(U)$ for combinations of $B S, S V$, and $C V$ that were tested at two or more velocities.

\subsubsection{Component Concentrations Plotted Versus Pre-Transfer and Transfer Batches}

Figure B.1 through Figure B.4 in Appendix B display plots of component concentrations (gibbsite, $\mathrm{SS}$, sand, and $\mathrm{ZrO}_{2}$, respectively) on the $y$-axis versus pre-transfer and five batch transfers on the $x$-axis. The data for all 26 test combinations in Table 2.3 and Table 2.4 are plotted, with separate plots for test combinations in the 43-inch and 120-inch tanks. The pre-transfer and five batch transfer values for a given test in a given tank are connected by the same color line segments to help visualize how the component concentrations change from pre-transfer through the five batch transfers. Each of the 26 test combinations has a different line color. The tests that are replicate pairs also have plotting symbols (of the same colors as the line segments), allowing for visual comparison of replicate tests. The plots for the 43-inch tank (on the left) and the 120-inch tank (on the right) are on the same page for a given component. This arrangement allows for easy comparison of the concentration traces (versus pre-transfer and five batch transfers) for the 26 test combinations for the two tanks. This arrangement also allows the two plots in a figure to share one legend.

Figure B.5 through Figure B.8 are similar to Figure B.1 through Figure B.4, except that the natural logarithms of the component concentrations are plotted on the $y$-axis. The natural logarithm transformation allows seeing the differences between traces for the Typical and High base simulants (which tend to be bunched together, especially for components that made up small proportions of either the Typical or High base simulant).

Based on the data shown in Figure B.1 through Figure B.4 and Figure B.5 through Figure B.8, the following observations are made regarding concentrations of gibbsite, SS, sand, and $\mathrm{ZrO}_{2}$ in pre-transfer and batch-transfer samples. In the following, the term batch is used to refer to pre-transfer as well as transfer batches. The term profile is used to refer to the relationship between the concentrations of the $j^{\text {th }}$ component $\left(C_{j}\right)$ as a function of batch.

1. Gibbsite concentrations were very low for $B S=$ High, while SS and sand concentrations were very low for $B S=$ Typical because these components were present as small fractions in those base simulants (see Table 2.2).

2. Figure B.1 and Figure B.5 show that there is limited variation in the $C_{\text {gibbsite }}$-batch profiles for each of the two base simulants. There are no obvious trends in $C_{\text {gibbsite }}$ or $\ln \left(C_{\text {gibbsite }}\right)$ as a function of batches for either of the two base simulants.

3. Figure B.2 shows that the variation in $C_{S S}$-batch profiles is much larger for tests with $B S=$ High than for tests with $B S=$ Typical. SS concentrations tend to be relatively flat with possibly a small decrease over batches for tests with $B S=$ Typical, but the decreasing trend with batch becomes 
more pronounced for the tests with $B S=$ High. Figure B.6 shows that the natural logarithm transformation tends to linearize the $\ln \left(C_{S S}\right)$-batch profiles.

4. Figure B.3 shows that the variation in $C_{\text {sand }}$-batch profiles is much larger for tests with $B S=$ High than for tests with $B S=$ Typical. Sand concentrations tend to be relatively flat for tests with $B S=$ Typical, but there are decreasing trends with batch for the tests with $B S=$ High. Figure B.7 shows that the natural logarithm transformation tends to linearize the $\ln \left(C_{\text {sand }}\right)$-batch profiles for tests with $B S=$ High, but some profiles still have curvilinear $\ln \left(C_{\text {sand }}\right)$-batch relationships.

5. Figure B.4 shows the $C_{\mathrm{ZrO} 2}$-batch profiles for tests with $B S=$ Typical are closer to the profiles for tests with $B S=$ High than for other components. This is consistent with the closer mass fractions for $\mathrm{ZrO}_{2}$ in the two base simulants (Table 2.2). Most of the profiles tend to be relatively flat, although a few profiles appear to have decreasing trends in $\mathrm{C}_{\mathrm{ZrO} 2}$ with increasing batch.

\subsubsection{Component Concentrations Plotted Versus Mixer-Jet Nozzle Velocity}

Seven combinations of $B S, S V$, and $C V$ were tested at two or more velocities, as listed in Table 3.3. Five of the seven combinations were tested at two velocities, Combination 1 was tested at three velocities, and Combination 7 was tested at four velocities. The plots for the seven combinations are shown in seven panels of Figure 3.1 through Figure 3.4, while the data for the 43-inch and 120-inch tanks are shown within each plot (panel). The values of $S V$ and $C V$ shown in the plots are the target values that were planned (see Table 2.3 and Table 2.4).

Table 3.3. Combinations of Base Simulant, Supernatant Viscosity, and Capture Velocity Tested at Two or More Mixer-Jet Nozzle Velocities

\begin{tabular}{ccccc}
\hline Combination & $\begin{array}{c}\text { Base } \\
\text { Simulant }\end{array}$ & $\begin{array}{c}\text { Target } \\
\text { Supernatant } \\
\text { Viscosity (cP) }\end{array}$ & $\begin{array}{c}\text { Target Capture } \\
\text { Velocity (ft/sec) }\end{array}$ & $\begin{array}{c}\text { Coded Velocities } \\
\text { Tested }^{(\mathbf{a})}\end{array}$ \\
\hline 1 & Typical & 3.3 & 3.8 & $-0.5,0,0.5$ \\
2 & Typical & 3.3 & 7.3 & $-0.5,1$ \\
3 & Typical & 8.0 & 7.3 & $-1,1$ \\
4 & High & 3.3 & 7.3 & $-1,0$ \\
5 & High & 8.0 & 3.8 & $-0.5,0.5$ \\
6 & High & 8.0 & 7.3 & $-1,1$ \\
7 & High & 14.6 & 11.3 & $-1,-0.5,0,1$ \\
\hline
\end{tabular}

(a) The coded velocities are $-1,-0.5,0,0.5$, and 1 , as discussed in Section 2.1.

In Figure 3.1 through Figure 3.4, the component concentration values for pre-transfer and batchtransfer samples are all plotted with log-scale axes. The lines in the figures represent separate power-law model fits to the data in each panel from the 43-inch and 120-inch tanks. The following notes explain how to interpret these plots.

(i) Slopes of Lines: Slopes of the lines in the plots provide certain information, but it should be kept in mind that only two of the seven panels in the plot have more than two velocity values. With only two velocity values having varying spreads, the lines corresponding to the power-law model 


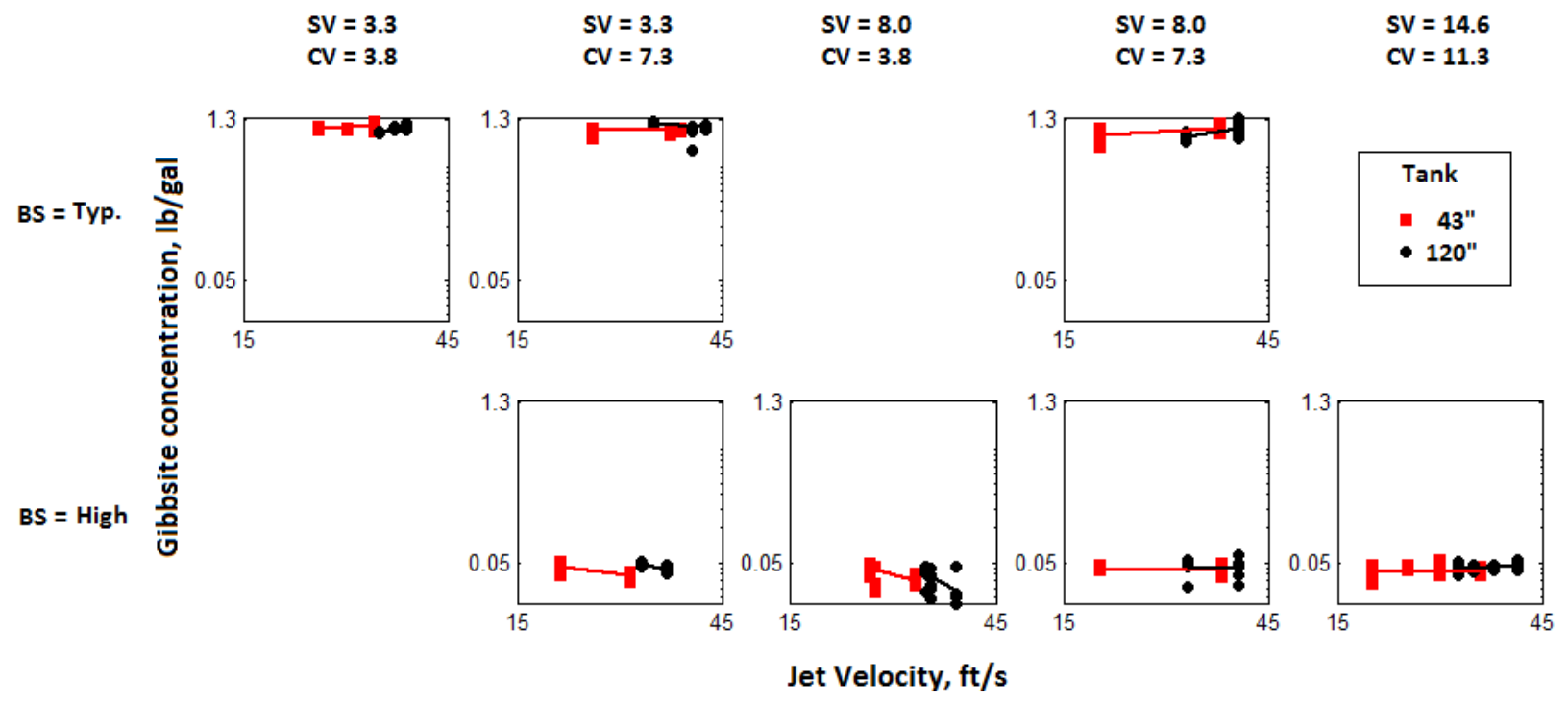

Figure 3.1. Plots of Gibbsite Concentrations versus Mixer-Jet Nozzle Velocity for 43-inch and 120-inch Tanks for Seven Test Combinations with at Least Two Velocities (For Information Only)

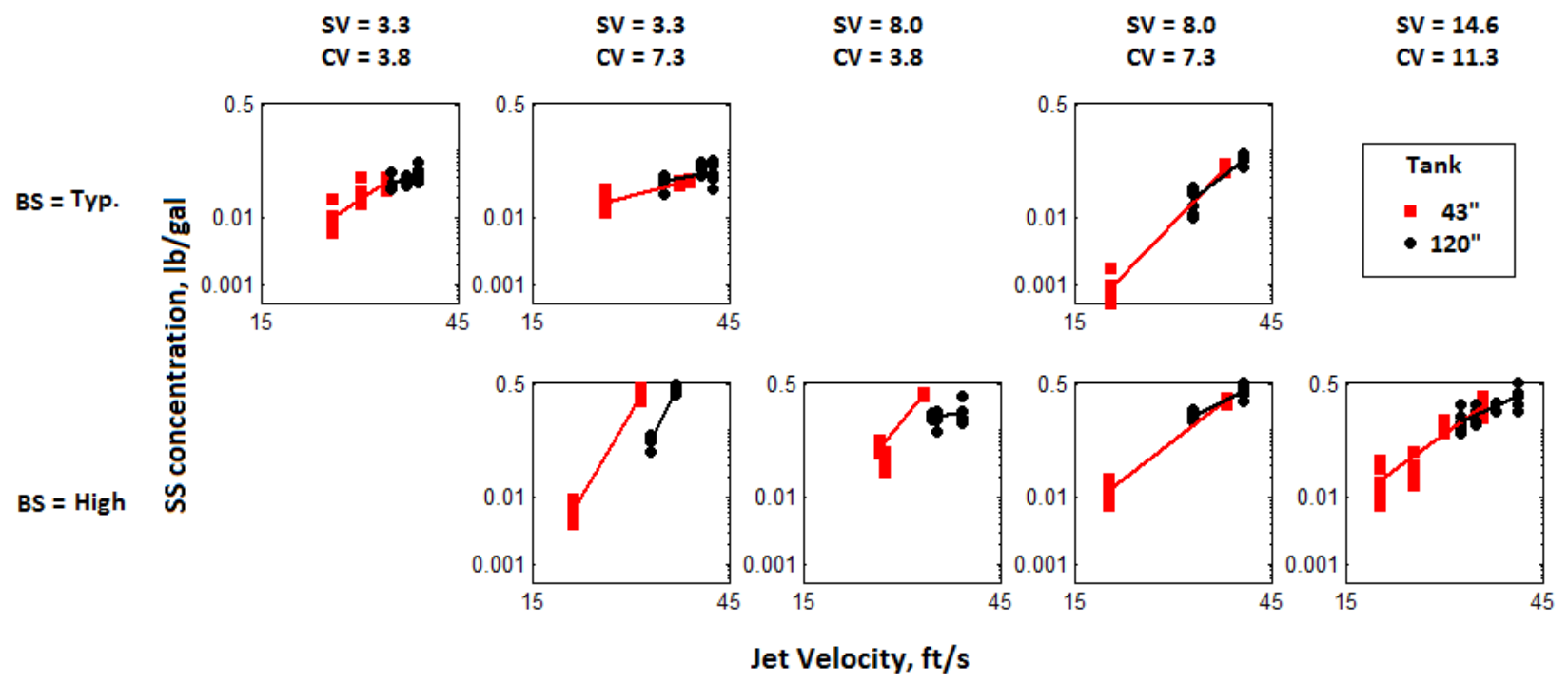

Figure 3.2. Plots of Stainless Steel Concentrations versus Mixer-Jet Nozzle Velocity for 43 -inch and 120-inch Tanks for Seven Test Combinations with at Least Two Velocities (For Information Only) 


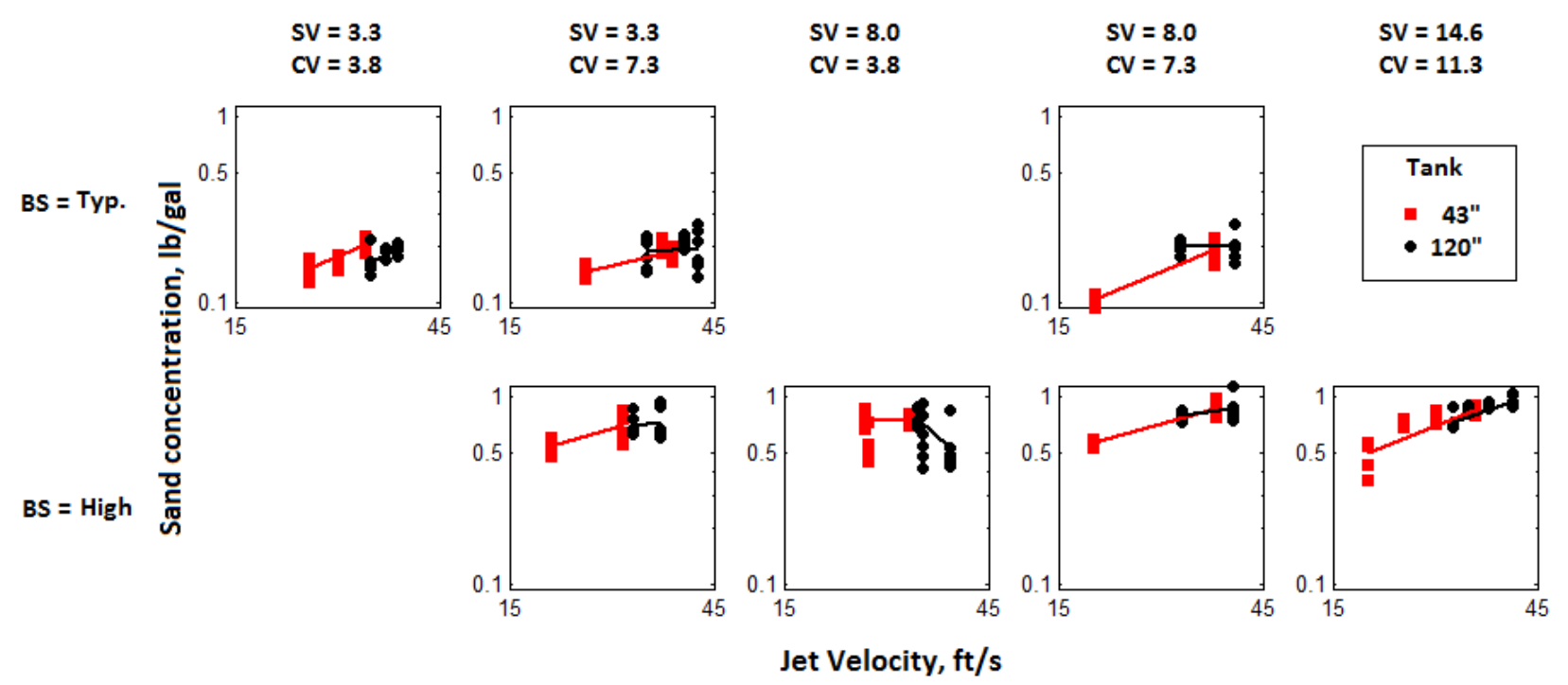

Figure 3.3. Plots of Sand Concentrations versus Mixer-Jet Nozzle Velocity for 43 -inch and 120 -inch Tanks for Seven Test Combinations with at Least Two Velocities (For Information Only)

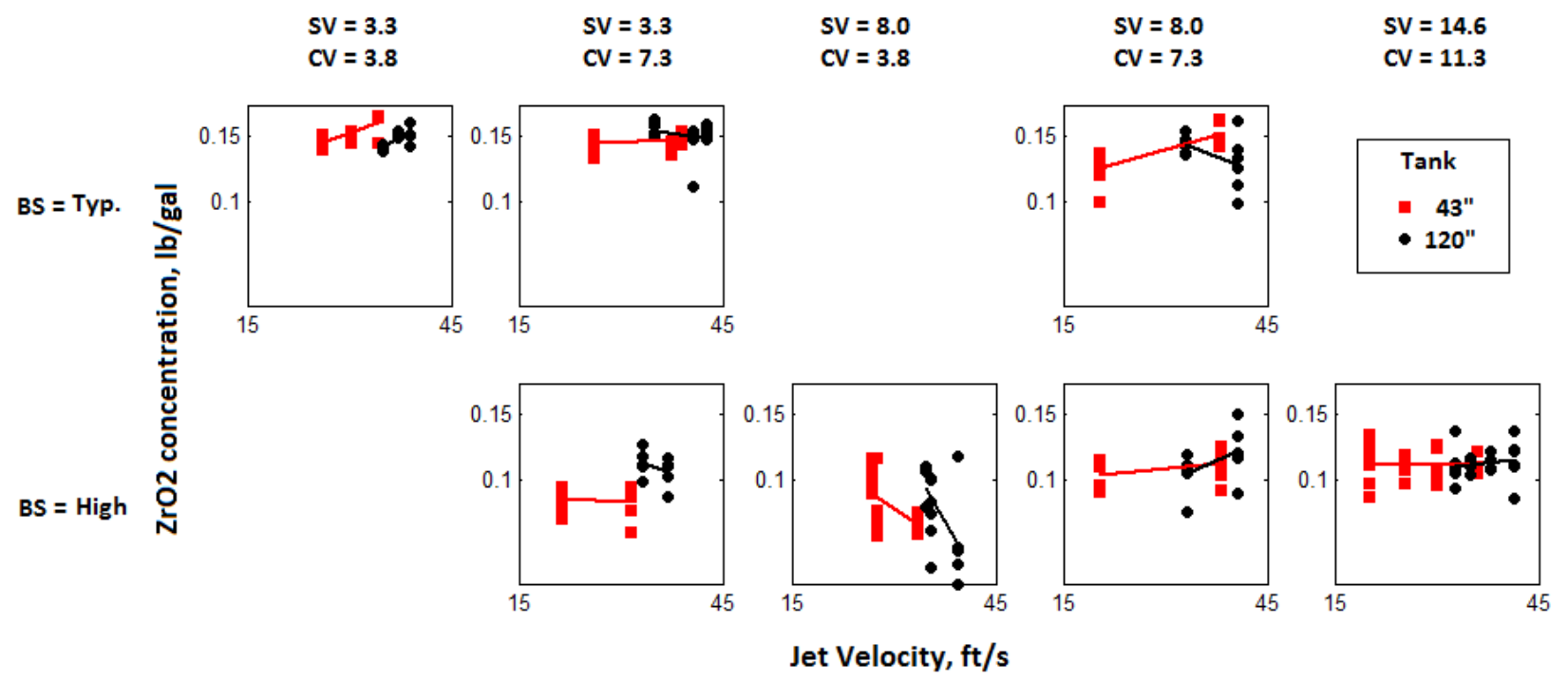

Figure 3.4. Plots of $\mathrm{ZrO}_{2}$ Concentrations versus Mixer-Jet Nozzle Velocity for 43-inch and 120-inch Tanks for Seven Test Combinations with at Least Two Velocities (For Information Only) 
fits can be quite uncertain. Also, there is uncertainty because of using all pre-transfer and batchtransfer data to fit the power-law models. Considering these uncertainties, lines with different slopes are interpreted as follows:

a. Roughly horizontal lines indicate there is negligible dependence of the component concentration on jet velocity.

b. Lines with positive slopes indicate that increasing velocity increases the component concentration.

c. Lines with negative slopes indicate that increasing velocity decreases the component concentration. This could happen in the following scenario. As jet velocity increases from zero, the solids at an elevation near the tank bottom (where transfers are made) can increase as more solids are suspended. Upon further increase in velocity, the solids at the bottom decrease as the height to which those solids are suspended increases. Lines with negative slopes occur only for gibbsite and $\mathrm{ZrO}_{2}$, which are generally expected to mix more easily than SS and sand, and hence are more likely to follow the preceding scenario. Still, note that some lines that appear to have negative slopes occur for data with a lot of uncertainty. Hence, some of the lines with negative slopes may be artifacts of limited data and uncertainty in the power-law fits.

(ii) Relative Positions of Lines for 43-inch and 120-inch Tanks: In a given panel of the plot, the relative positions of the lines for the 43-inch and 120-inch tanks provide information about the scaling exponent for the set of conditions represented by that panel.

a. Lines that are approximately parallel indicate that the strength of the dependence of the component concentration on velocity is approximately the same for the 43-inch and 120-inch tanks. Lines that are not approximately parallel indicate that the strength of the dependence of the component concentration on velocity is not the same for the 43-inch and 120-inch tanks.

b. Lines for the 43-inch and 120-inch tanks that have similar slopes and intercepts indicate that the scaling exponent is approximately 0 .

c. A positive scaling exponent is indicated when the lines for the two tanks are approximately parallel with positive slopes and the line for the 120 -inch tank has a smaller $y$-intercept than the line for the 43-inch tank. In such cases, a higher velocity in the 120-inch tank than in the 43 -inch tank is required to achieve the same component concentration.

d. A negative scaling exponent is indicated when the lines for the two tanks are approximately parallel with positive slopes and the line for the 120-inch tank has a larger $y$-intercept than the line for the 43-inch tank. In such cases, a lower velocity in the 120-inch tank than in the 43-inch tank is required to achieve the same component concentration.

e. Lines with positive slopes that are not approximately parallel indicate that the scaling exponent could be positive or negative, depending on the velocity at which the lines intersect. Above the velocity where the lines intersect, the scaling exponent is positive because a larger velocity is required in the 120 -inch tank than in the 43 -inch tank to achieve a given component concentration. Below the velocity where the lines intersect, the scaling exponent 
is negative because a smaller velocity is required in the 120 -inch tank than in the 43 -inch tank to achieve a given component concentration.

For gibbsite, Figure 3.1 shows the lines for the 43-inch and 120-inch tanks have similar slopes, roughly equal to zero [see Item (i)a] with one exception. For the case $(B S=\mathrm{H}, S V=8.0, C V=7.3)$, the lines appear to have positive slopes with the line for the 120 -inch tank having a slightly smaller intercept [see Item (ii)c]. In general, these plots suggest there is no effect of velocity on gibbsite concentrations, so that no velocity scaling is possible.

For SS, Figure 3.2 shows that the lines generally have negligible to positive slopes, with the exception of the 120-inch tank for $B S=$ High, $S V=8.0$, and $C V=3.8$, which has a negative slope (although with some uncertainty because of the scatter in the data points). Because of the similarity of the slopes and intercepts, these plots indicate that the scaling exponent is probably no more than 0.10 to 0.15 .

For sand, Figure 3.3 shows that the lines generally have similar slopes that are positive, although some of the plots indicate a possibility of different slopes. In particular, the case of $B S=$ High, $S V=8.0$, and $C V=3.8$ has what appears to be different slopes, although the two data points were the highest of the two coded velocities tested. The case of $B S=$ High, $S V=3.3$, and $C V=7.3$ is also of special note because it has lines with similar slopes and the largest difference in $y$-intercepts, which corresponds to a scaling exponent of 0.20 to 0.25 .

For $\mathrm{ZrO}_{2}$, Figure 3.4 shows a range of slopes from negative to negligible to positive. The variation in the different panels may be because $\mathrm{ZrO}_{2}$ is expected to mix relatively easily and what is being observed is experimental and measurement uncertainty.

\subsection{Estimates of Uncertainties in Component Concentrations Based on Replicate Tests}

As discussed in Section 2.2, a total of eight replicate tests were performed, four each for the 43-inch tank and the 120-inch tank. The eight pairs of replicate tests provide for quantifying the combined uncertainty from all sources of variation included in the replicate tests (e.g., experimental, sampling, and analytical).

Table 3.4 through Table 3.7 list the standard deviations (SD) and percent relative standard deviations (\%RSD) of component (gibbsite, SS, sand, and $\mathrm{ZrO}_{2}$, respectively) concentrations for each replicate pair as well as summaries of the SDs and \%RSDs (min, pooled, max). Here "pooled" refers to a combined estimate of separate estimates of a SD or \%RSD. Pooled estimates are calculated by taking the square root of the weighted average of the individual SD or \%RSD values squared. The equation for this is Pooled $(S D)=\sqrt{\frac{\sum\left(n_{i}-1\right)\left(S D_{i}\right)^{2}}{\sum\left(n_{i}-1\right)}}$ with the summations taken over the number of groups that are being

pooled. In this case there were replicate pairs, so $n_{i}=2$ for all groups $i$. The equation is the same for $\%$ RSD with SD replaced with \%RSD everywhere in the equation. 
Table 3.4. Gibbsite $\left[\mathrm{Al}(\mathrm{OH})_{3}\right]$ Concentrations (lb/gal), Standard Deviations, and Percent Relative Standard Deviations for Pre-Transfer and Batch-Transfer Samples from Replicate Tests (For Information Only)

\begin{tabular}{|c|c|c|c|c|c|c|c|c|c|}
\hline $\begin{array}{l}\text { Replicate } \\
\text { Pair Test } \\
\text { Numbers }\end{array}$ & $\begin{array}{c}\text { Pre- } \\
\text { Transfer }\end{array}$ & Batch 1 & Batch 2 & Batch 3 & Batch 4 & Batch 5 & Min & $\begin{array}{l}\text { Pooled } \\
\text { (a) }\end{array}$ & Max \\
\hline \multicolumn{10}{|c|}{ 43-inch Tank } \\
\hline 4 & 0.9467 & 1.0442 & 1.0394 & 1.0613 & 1.0448 & 1.0487 & - & - & - \\
\hline 5 & 1.0646 & 1.0887 & 1.0697 & 1.0412 & 1.0082 & 1.0583 & - & - & - \\
\hline SD & 0.0834 & 0.0314 & 0.0214 & 0.0142 & 0.0258 & 0.0068 & 0.0068 & 0.0394 & 0.0834 \\
\hline$\%$ RSD & 8.29 & 2.94 & 2.03 & 1.35 & 2.52 & 0.64 & 1.35 & 3.88 & 8.29 \\
\hline 6 & 0.0490 & 0.0498 & 0.0375 & 0.0444 & 0.0436 & 0.0416 & - & - & - \\
\hline 7 & 0.0458 & 0.0289 & 0.0323 & 0.0273 & 0.0321 & 0.0315 & - & - & - \\
\hline SD & 0.0022 & 0.0148 & 0.0037 & 0.0121 & 0.0082 & 0.0072 & 0.0022 & 0.0091 & 0.0148 \\
\hline$\%$ RSD & 4.74 & 37.62 & 10.61 & 33.63 & 21.58 & 19.57 & 4.74 & 24.26 & 37.62 \\
\hline 11 & 0.0474 & 0.0462 & 0.0467 & 0.0426 & 0.0389 & 0.0416 & - & - & - \\
\hline 12 & 0.0432 & 0.0426 & 0.0469 & 0.0472 & 0.0437 & 0.0456 & - & - & - \\
\hline & 0.0030 & 0.0026 & 0.00016 & 0.0033 & 0.0034 & 0.0028 & 0.00016 & 0.0028 & 0.0034 \\
\hline$\%$ RSD & 6.54 & 5.82 & 0.33 & 7.26 & 8.21 & 6.46 & 0.33 & 6.31 & 8.21 \\
\hline 16 & 1.0889 & 1.1326 & 1.1413 & 1.1713 & 1.1374 & 1.1603 & - & - & - \\
\hline 17 & 1.0154 & 1.0114 & 1.0425 & 1.0272 & 1.0656 & 1.1101 & - & - & - \\
\hline & 0.0520 & 0.0857 & 0.0699 & 0.1019 & 0.0508 & 0.0356 & 0.0356 & 0.0697 & 0.1019 \\
\hline$\%$ RSD & 4.94 & 8.00 & 6.40 & 9.27 & 4.61 & 3.13 & 4.61 & 6.41 & 9.27 \\
\hline \multicolumn{10}{|c|}{ 120-inch Tank } \\
\hline 4 & 1.0592 & 1.1776 & 1.1253 & 1.0871 & 1.1571 & 1.0833 & - & - & - \\
\hline 5 & 0.6947 & 1.1141 & 1.0444 & 1.0238 & 1.1098 & 1.0705 & - & - & - \\
\hline SD & 0.2577 & 0.0449 & 0.0572 & 0.0448 & 0.0334 & 0.0090 & 0.0090 & 0.1117 & 0.2577 \\
\hline$\%$ RSD & 29.39 & 3.92 & 5.28 & 4.24 & 2.95 & 0.84 & 2.95 & 12.48 & 29.39 \\
\hline 6 & 0.0425 & 0.0408 & 0.0272 & 0.0400 & 0.0426 & 0.0460 & - & - & - \\
\hline 7 & 0.0447 & 0.0395 & 0.0362 & 0.0315 & 0.0293 & 0.0237 & - & - & - \\
\hline SD & 0.0016 & 0.00089 & 0.0063 & 0.0060 & 0.0094 & 0.0158 & 0.00089 & 0.0083 & 0.0158 \\
\hline$\%$ RSD & 3.60 & 2.22 & 20.04 & 16.86 & 26.24 & 45.27 & 2.22 & 23.95 & 45.27 \\
\hline 11 & 0.0475 & 0.0457 & 0.0480 & 0.0513 & 0.0484 & 0.0537 & - & - & - \\
\hline 12 & 0.0514 & 0.0477 & 0.0477 & 0.0488 & 0.0486 & 0.0500 & - & - & - \\
\hline SD & 0.0027 & 0.0014 & 0.00024 & 0.0018 & 0.00012 & 0.0026 & 0.00012 & 0.0018 & 0.0027 \\
\hline$\%$ RSD & 5.45 & 2.94 & 0.50 & 3.57 & 0.25 & 4.96 & 0.25 & 3.56 & 5.45 \\
\hline 16 & 1.0246 & 1.0697 & 1.0742 & 1.0399 & 1.0093 & 1.0649 & - & - & - \\
\hline 17 & 1.0761 & 1.0464 & 1.0523 & 1.0607 & 1.0538 & 1.0749 & - & - & - \\
\hline SD & 0.0364 & 0.0165 & 0.0155 & 0.0147 & 0.0315 & 0.0070 & 0.0070 & 0.0227 & 0.0364 \\
\hline$\%$ RSD & 3.47 & 1.56 & 1.46 & 1.40 & 3.05 & 0.66 & 1.40 & 2.17 & 3.47 \\
\hline \multicolumn{10}{|c|}{ Summary Over Replicate Pairs for Both Tanks } \\
\hline Min SD & 0.0016 & 0.00089 & 0.00016 & 0.0018 & 0.00012 & 0.0026 & 0.00012 & - & - \\
\hline Pooled SD & 0.0984 & 0.0368 & 0.0334 & 0.0403 & 0.0263 & 0.0148 & - & 0.0495 & - \\
\hline Max SD & 0.2577 & $\mathbf{0 . 0 8 5 7}$ & 0.0699 & 0.1019 & 0.0508 & 0.0356 & - & - & 0.2577 \\
\hline Min \%RSD & 3.47 & 1.56 & 0.33 & 1.35 & 0.25 & 0.64 & 0.25 & - & - \\
\hline Pooled & 11.60 & 13.93 & 8.59 & 14.09 & 12.59 & 17.71 & - & 13.37 & - \\
\hline Max \%RSD & 29.39 & 37.62 & 20.04 & 33.63 & 26.24 & 45.27 & - & - & 45.27 \\
\hline
\end{tabular}


Table 3.5. Stainless Steel Concentrations (lb/gal), Standard Deviations, and Percent Relative Standard Deviations for Pre-Transfer and Batch-Transfer Samples from Replicate Tests (For Information Only)

\begin{tabular}{|c|c|c|c|c|c|c|c|c|c|}
\hline $\begin{array}{l}\text { Replicate } \\
\text { Pair Test } \\
\text { Numbers }\end{array}$ & $\begin{array}{c}\text { Pre- } \\
\text { Transfer }\end{array}$ & Batch 1 & Batch 2 & Batch 3 & Batch 4 & Batch 5 & Min & $\begin{array}{l}\text { Pooled } \\
\text { (a) }\end{array}$ & Max \\
\hline \multicolumn{10}{|c|}{ 43-inch Tank } \\
\hline 4 & 0.0299 & 0.0322 & 0.0359 & 0.0331 & 0.0360 & 0.0326 & - & - & - \\
\hline 5 & 0.0376 & 0.0376 & 0.0337 & 0.0362 & 0.0333 & 0.0339 & - & - & - \\
\hline SD & 0.0054 & 0.0038 & 0.0015 & 0.0022 & 0.0019 & 0.00093 & 0.00093 & 0.0030 & 0.0054 \\
\hline$\%$ RSD & 15.99 & 10.89 & 4.42 & 6.26 & 5.53 & 2.81 & 4.42 & 8.86 & 15.99 \\
\hline 6 & 0.0733 & 0.0550 & 0.0513 & 0.0442 & 0.0463 & 0.0566 & - & - & - \\
\hline 7 & 0.0470 & 0.0468 & 0.0385 & 0.0283 & 0.0241 & 0.0348 & - & - & - \\
\hline SD & 0.0186 & 0.0058 & 0.0091 & 0.0113 & 0.0157 & 0.0154 & 0.0058 & 0.0134 & 0.0186 \\
\hline$\%$ RSD & 30.85 & 11.34 & 20.24 & 31.10 & 44.56 & 33.79 & 11.34 & 30.51 & 44.56 \\
\hline 11 & 0.1041 & 0.1255 & 0.1116 & 0.1116 & 0.0959 & 0.0822 & - & - & - \\
\hline 12 & 0.1054 & 0.1275 & 0.1219 & 0.1278 & 0.1474 & 0.1499 & - & - & - \\
\hline & 0.00093 & 0.0015 & 0.0073 & 0.0115 & 0.0364 & 0.0479 & 0.00093 & 0.0252 & 0.0479 \\
\hline$\%$ RSD & 0.89 & 1.15 & 6.27 & 9.58 & 29.95 & 41.27 & 0.89 & 21.34 & 41.27 \\
\hline 16 & 0.0552 & 0.0399 & 0.0338 & 0.0333 & 0.0256 & 0.0241 & - & - & - \\
\hline 17 & 0.0320 & 0.0088 & 0.0102 & 0.0122 & 0.0159 & 0.0203 & - & - & - \\
\hline & 0.0164 & 0.0220 & 0.0167 & 0.0149 & 0.0069 & 0.0027 & 0.0027 & 0.0148 & 0.0220 \\
\hline$\%$ RSD & 37.54 & 90.34 & 76.09 & 65.50 & 32.97 & 12.15 & 32.97 & 59.00 & 90.34 \\
\hline \multicolumn{10}{|c|}{ 120-inch Tank } \\
\hline 4 & 0.0708 & 0.0612 & 0.0414 & 0.0372 & 0.0276 & 0.0465 & - & - & - \\
\hline 5 & 0.0697 & 0.0571 & 0.0655 & 0.0550 & 0.0568 & 0.0429 & - & - & - \\
\hline & 0.00076 & 0.0029 & 0.0171 & 0.0126 & 0.0207 & 0.0026 & 0.00076 & 0.0122 & 0.0207 \\
\hline$\%$ RSD & 1.08 & 4.88 & 31.91 & 27.25 & 48.95 & 5.76 & 1.08 & 26.51 & 48.95 \\
\hline 6 & 0.1880 & 0.1886 & 0.1481 & 0.1639 & 0.1623 & 0.1417 & - & - & - \\
\hline 7 & 0.1913 & 0.1752 & 0.1540 & 0.1428 & 0.1519 & 0.0943 & - & - & - \\
\hline SD & 0.0023 & 0.0095 & 0.0041 & 0.0149 & 0.0073 & 0.0335 & 0.0023 & 0.0159 & 0.0335 \\
\hline$\%$ RSD & 1.23 & 5.23 & 2.73 & 9.71 & 4.67 & 28.40 & 1.23 & 12.64 & 28.40 \\
\hline 11 & 0.3305 & 0.3113 & 0.2457 & 0.2016 & 0.1619 & 0.0927 & - & - & - \\
\hline 12 & 0.2329 & 0.2689 & 0.2707 & 0.2369 & 0.2663 & 0.1902 & - & - & - \\
\hline SD & 0.0690 & 0.0300 & 0.0177 & 0.0249 & 0.0738 & 0.0689 & 0.0177 & 0.0529 & 0.0738 \\
\hline$\%$ RSD & 24.50 & 10.34 & 6.85 & 11.36 & 34.49 & 48.74 & 6.85 & 27.23 & 48.74 \\
\hline 16 & 0.0448 & 0.0516 & 0.0498 & 0.0404 & 0.0387 & 0.0353 & - & - & - \\
\hline 17 & 0.0578 & 0.0510 & 0.0474 & 0.0473 & 0.0349 & 0.0325 & - & - & - \\
\hline SD & 0.0092 & 0.00040 & 0.0017 & 0.0049 & 0.0027 & 0.0019 & 0.00040 & 0.0045 & 0.0092 \\
\hline$\%$ RSD & $\mathbf{1 7 . 8 8}$ & 0.79 & 3.51 & 11.13 & 7.44 & 5.64 & 0.79 & 9.52 & 17.88 \\
\hline \multicolumn{10}{|c|}{ Summary Over Replicate Pairs for Both Tanks } \\
\hline Min SD & 0.00076 & 0.00040 & 0.0015 & 0.0022 & 0.0019 & 0.00093 & 0.00040 & - & - \\
\hline Pooled SD & 0.0262 & 0.0138 & 0.0114 & 0.0137 & 0.0308 & 0.0325 & - & 0.0231 & - \\
\hline Max SD & 0.0690 & 0.0300 & 0.0177 & 0.0249 & 0.0738 & 0.0689 & - & - & 0.0738 \\
\hline Min \%RSD & 0.89 & 0.79 & 2.73 & 6.26 & 4.67 & 2.81 & 0.79 & - & - \\
\hline Pooled & 21.04 & 32.73 & 30.30 & 28.46 & 30.95 & 27.94 & - & 28.81 & - \\
\hline Max \%RSD & 37.54 & 90.34 & 76.09 & 65.50 & 48.95 & 48.74 & - & - & 90.34 \\
\hline
\end{tabular}

(a) "Pooled" refers to a combining separate uncertainty estimates into one estimate. 
Table 3.6. Sand Concentrations (lb/gal), Standard Deviations, and Percent Relative Standard Deviations for Pre-Transfer and Batch-Transfer Samples from Replicate Tests (For Information Only)

\begin{tabular}{|c|c|c|c|c|c|c|c|c|c|}
\hline $\begin{array}{l}\text { Replicate } \\
\text { Pair Test } \\
\text { Numbers }\end{array}$ & $\begin{array}{c}\text { Pre- } \\
\text { Transfer }\end{array}$ & Batch 1 & Batch 2 & Batch 3 & Batch 4 & Batch 5 & Min & $\begin{array}{l}\text { Pooled } \\
\text { (a) }\end{array}$ & Max \\
\hline \multicolumn{10}{|c|}{ 43-inch Tank } \\
\hline 4 & 0.2238 & 0.1888 & 0.2015 & 0.2026 & 0.1846 & 0.1843 & - & - & - \\
\hline 5 & 0.1675 & 0.1976 & 0.1995 & 0.1975 & 0.1913 & 0.1820 & - & - & - \\
\hline SD & 0.0398 & 0.0063 & 0.0015 & 0.0036 & 0.0048 & 0.0016 & 0.0015 & 0.0167 & 0.0398 \\
\hline$\%$ RSD & 20.37 & 3.24 & 0.73 & 1.80 & 2.53 & 0.89 & $\mathbf{0 . 7 3}$ & 8.53 & 20.37 \\
\hline 6 & 0.8772 & 0.7926 & 0.7039 & 0.6981 & 0.6818 & 0.7448 & - & - & - \\
\hline 7 & 0.7340 & 0.5158 & 0.5636 & 0.4525 & 0.4862 & 0.4975 & - & - & - \\
\hline SD & 0.1013 & 0.1957 & 0.0992 & 0.1737 & 0.1383 & 0.1748 & 0.0992 & 0.1518 & 0.1957 \\
\hline$\%$ RSD & 12.57 & 29.92 & 15.65 & 30.19 & 23.68 & 28.15 & 12.57 & 24.37 & 30.19 \\
\hline 11 & 0.8208 & 0.6969 & 0.6940 & 0.7215 & 0.7086 & 0.7112 & - & - & - \\
\hline 12 & 0.9016 & 0.8271 & 0.8135 & 0.7786 & 0.7990 & 0.7992 & - & - & - \\
\hline & 0.0571 & 0.0920 & 0.0845 & 0.0404 & 0.0639 & 0.0622 & 0.0404 & 0.0689 & 0.0920 \\
\hline$\%$ RSD & 6.63 & 12.08 & 11.22 & 5.39 & 8.48 & 8.24 & 5.39 & 8.99 & 12.08 \\
\hline 16 & 0.1954 & 0.2013 & 0.2215 & 0.2418 & 0.2012 & 0.2335 & - & - & - \\
\hline 17 & 0.1745 & 0.1660 & 0.1753 & 0.1851 & 0.1755 & 0.1645 & - & - & - \\
\hline SD & 0.0148 & 0.0250 & 0.0327 & 0.0401 & 0.0181 & 0.0487 & 0.0148 & 0.0322 & 0.0487 \\
\hline$\%$ RSD & 8.00 & 13.59 & 16.48 & 18.79 & 9.62 & 24.49 & 8.00 & 16.15 & 24.49 \\
\hline \multicolumn{10}{|c|}{ 120-inch Tank } \\
\hline 4 & 0.2159 & 0.2422 & 0.1596 & 0.1705 & 0.1365 & 0.2638 & - & - & - \\
\hline 5 & 0.2093 & 0.2171 & 0.2352 & 0.2125 & 0.1994 & 0.1909 & - & - & - \\
\hline & 0.0046 & 0.0177 & 0.0534 & 0.0297 & 0.0445 & 0.0516 & 0.0046 & 0.0381 & 0.0534 \\
\hline$\%$ RSD & 2.18 & 7.73 & 27.06 & 15.51 & 26.47 & 22.68 & 2.18 & 19.37 & 27.06 \\
\hline 6 & 0.8865 & 0.8780 & 0.6899 & 0.7348 & 0.7073 & 0.7461 & - & - & - \\
\hline 7 & 0.9237 & 0.8026 & 0.6346 & 0.5443 & 0.4816 & 0.4204 & - & - & - \\
\hline SD & 0.0263 & 0.0533 & 0.0391 & 0.1347 & 0.1596 & 0.2304 & 0.0263 & 0.1302 & 0.2304 \\
\hline$\%$ RSD & 2.91 & 6.34 & 5.91 & 21.06 & 26.85 & 39.50 & 2.91 & 21.63 & 39.50 \\
\hline 11 & 0.9830 & 0.8935 & 0.8256 & 0.8099 & 0.7876 & 0.8104 & - & - & - \\
\hline 12 & 1.0010 & 0.9843 & 0.8407 & 0.8134 & 0.7755 & 0.8536 & - & - & - \\
\hline SD & 0.0127 & 0.0642 & 0.0106 & 0.0025 & 0.0086 & 0.0306 & 0.0025 & 0.0300 & 0.0642 \\
\hline$\%$ RSD & 1.28 & 6.84 & 1.27 & $\mathbf{0 . 3 0}$ & 1.09 & 3.67 & 0.30 & 3.29 & 6.84 \\
\hline 16 & 0.1669 & 0.2214 & 0.2122 & 0.2045 & 0.1750 & 0.1843 & - & - & - \\
\hline 17 & 0.2093 & 0.1948 & 0.2157 & 0.2144 & 0.2538 & 0.2039 & - & - & - \\
\hline SD & 0.0300 & 0.0188 & 0.0025 & 0.0070 & 0.0557 & 0.0138 & 0.0025 & $\mathbf{0 . 0 2 7 7}$ & 0.0557 \\
\hline$\%$ RSD & 15.95 & 9.05 & 1.17 & 3.35 & 26.00 & 7.12 & 1.17 & 13.39 & 26.00 \\
\hline \multicolumn{10}{|c|}{ Summary Over Replicate Pairs for Both Tanks } \\
\hline Min SD & 0.0046 & 0.0063 & 0.0015 & 0.0025 & 0.0048 & 0.0016 & 0.0015 & - & - \\
\hline Pooled SD & 0.0462 & 0.0830 & 0.0531 & 0.0810 & 0.0823 & 0.1082 & - & 0.0784 & - \\
\hline Max SD & 0.1013 & 0.1957 & 0.0992 & 0.1737 & 0.1596 & 0.2304 & - & - & 0.2304 \\
\hline Min \%RSD & 1.28 & 3.24 & 0.73 & 0.30 & 1.09 & 0.89 & 0.30 & - & - \\
\hline Pooled & 10.90 & 13.53 & 13.29 & 15.78 & 18.81 & 21.21 & - & 15.97 & - \\
\hline Max \%RSD & 20.37 & 29.92 & 27.06 & 30.19 & 26.85 & 39.50 & - & - & 39.50 \\
\hline
\end{tabular}


Table 3.7. $\mathrm{ZrO}_{2}$ Concentrations (lb/gal), Standard Deviations, and Percent Relative Standard Deviations for Pre-Transfer and Batch-Transfer Samples from Replicate Tests (For Information Only)

\begin{tabular}{|c|c|c|c|c|c|c|c|c|c|}
\hline $\begin{array}{l}\text { Replicate } \\
\text { Pair Test } \\
\text { Numbers }\end{array}$ & $\begin{array}{c}\text { Pre- } \\
\text { Transfer }\end{array}$ & Batch 1 & Batch 2 & Batch 3 & Batch 4 & Batch 5 & Min & $\begin{array}{l}\text { Pooled } \\
\text { (a) }\end{array}$ & Max \\
\hline \multicolumn{10}{|c|}{ 43-inch Tank } \\
\hline 4 & 0.1333 & 0.1435 & 0.1434 & 0.1454 & 0.1438 & 0.1432 & - & - & - \\
\hline 5 & 0.1529 & 0.1550 & 0.1535 & 0.1518 & 0.1428 & 0.1532 & - & - & - \\
\hline SD & 0.0139 & 0.0082 & 0.0071 & 0.0046 & 0.00070 & 0.0071 & 0.00070 & 0.0080 & 0.0139 \\
\hline$\%$ RSD & 9.69 & 5.48 & 4.77 & 3.08 & 0.49 & 4.77 & 0.49 & 5.47 & 9.69 \\
\hline 6 & 0.1153 & 0.1081 & 0.0931 & 0.1004 & 0.0966 & 0.0992 & - & - & - \\
\hline 7 & 0.1151 & 0.0759 & 0.0839 & 0.0717 & 0.0774 & 0.0777 & - & - & - \\
\hline SD & 0.00017 & 0.0228 & 0.0064 & 0.0203 & 0.0136 & 0.0152 & 0.00017 & 0.0152 & 0.0228 \\
\hline$\%$ RSD & 0.14 & 24.76 & 7.28 & 23.59 & 15.66 & 17.19 & 0.14 & 17.14 & 24.76 \\
\hline 11 & 0.1228 & 0.1043 & 0.1075 & 0.1031 & 0.0985 & 0.1073 & - & - & - \\
\hline 12 & 0.0996 & 0.1043 & 0.1131 & 0.1079 & 0.1020 & 0.1083 & - & - & - \\
\hline & 0.0164 & 0.00002 & 0.0040 & 0.0034 & 0.0024 & 0.00070 & 0.00002 & 0.0071 & 0.0164 \\
\hline$\%$ RSD & 14.74 & 0.023 & 3.58 & 3.22 & 2.42 & 0.65 & 0.023 & 6.42 & 14.74 \\
\hline 16 & 0.1521 & 0.1488 & 0.1536 & 0.1634 & 0.1525 & 0.1593 & - & - & - \\
\hline 17 & 0.1444 & 0.1350 & 0.1383 & 0.1382 & 0.1451 & 0.1548 & - & - & - \\
\hline SD & 0.0054 & 0.0098 & 0.0108 & 0.0178 & 0.0052 & 0.0032 & 0.0032 & 0.0100 & 0.0178 \\
\hline$\%$ RSD & 3.67 & 6.88 & 7.42 & 11.84 & 3.51 & 2.02 & 3.51 & 6.74 & 11.84 \\
\hline \multicolumn{10}{|c|}{ 120-inch Tank } \\
\hline 4 & 0.1474 & 0.1605 & 0.1541 & 0.1496 & 0.1575 & 0.1488 & - & - & - \\
\hline 5 & 0.1101 & 0.1540 & 0.1485 & 0.1472 & 0.1536 & 0.1494 & - & - & - \\
\hline & 0.0264 & 0.0046 & 0.0039 & 0.0017 & 0.0028 & 0.00044 & 0.00044 & 0.0111 & 0.0264 \\
\hline$\%$ RSD & 20.48 & 2.94 & 2.59 & 1.12 & 1.77 & $\mathbf{0 . 3 0}$ & 1.12 & 8.55 & 20.48 \\
\hline 6 & 0.1055 & 0.1091 & 0.0851 & 0.1061 & 0.1092 & 0.1080 & - & - & - \\
\hline 7 & 0.1007 & 0.1018 & 0.0878 & 0.0816 & 0.0736 & 0.0592 & - & - & - \\
\hline SD & 0.0034 & 0.0051 & 0.0018 & 0.0173 & 0.0252 & 0.0345 & 0.0018 & 0.0190 & 0.0345 \\
\hline$\%$ RSD & 3.28 & 4.84 & 2.13 & 18.45 & 27.53 & 41.29 & 2.13 & 21.76 & 41.29 \\
\hline 11 & 0.1120 & 0.1054 & 0.1127 & 0.1294 & 0.1176 & 0.1245 & - & - & - \\
\hline 12 & 0.1206 & 0.1105 & 0.1211 & 0.1185 & 0.1193 & 0.1169 & - & - & - \\
\hline SD & 0.0061 & 0.0036 & 0.0060 & 0.0077 & 0.0012 & 0.0054 & 0.0012 & 0.0054 & 0.0077 \\
\hline$\%$ RSD & 5.28 & 3.35 & 5.13 & 6.20 & 1.02 & 4.44 & 1.02 & 4.56 & 6.20 \\
\hline 16 & 0.1420 & 0.1662 & 0.1568 & 0.1498 & 0.1468 & 0.1507 & - & - & - \\
\hline 17 & 0.1549 & 0.1525 & 0.1529 & 0.1516 & 0.1652 & 0.1522 & - & - & - \\
\hline SD & 0.0091 & 0.0097 & 0.0028 & 0.0013 & 0.0130 & 0.0011 & 0.0011 & 0.0077 & 0.0130 \\
\hline$\%$ RSD & 6.14 & 6.07 & 1.79 & 0.84 & 8.34 & 0.70 & 0.84 & 4.97 & 8.34 \\
\hline \multicolumn{10}{|c|}{ Summary Over Replicate Pairs for Both Tanks } \\
\hline Min SD & 0.00017 & 0.00002 & 0.0018 & 0.0013 & $\mathbf{0 . 0 0 0 7 0}$ & 0.00044 & 0.00002 & - & - \\
\hline Pooled SD & 0.0128 & 0.0102 & 0.0060 & 0.0119 & 0.0113 & 0.0138 & - & 0.0113 & - \\
\hline Max SD & 0.0264 & 0.0228 & 0.0108 & 0.0203 & 0.0252 & 0.0345 & - & - & 0.0345 \\
\hline Min \%RSD & 0.14 & 0.023 & 1.79 & 0.84 & 0.49 & 0.30 & 0.023 & - & - \\
\hline Pooled & 10.13 & 9.82 & 4.80 & 11.71 & 11.70 & 16.00 & - & 11.20 & - \\
\hline Max \%RSD & 20.48 & 24.76 & 7.42 & 23.59 & 27.53 & 41.29 & - & - & 41.29 \\
\hline
\end{tabular}


Appendix C displays two kinds of graphical evaluations of the SDs and \%RSDs of component concentrations based on replicate pairs. Figure C.1 through Figure C.4 display, for concentrations of the four components (gibbsite, SS, sand, and $\mathrm{ZrO} 2$, respectively), the SDs and \%RSDs from replicate pairs versus batches (pre-transfer and five batch transfers). Different colors of lines are used for the replicate pairs. The SDs and \%RSDs for the 43-inch and 120-inch tanks share the same color line for a replicate pair, with the line being solid for the 43-inch tank and dashed for the 120-inch tank. Figure C.5 through Figure C.8 display the SDs versus the means of component concentrations from the replicate pairs, with different plotting symbols for 43-inch and 120-inch tanks. It should be kept in mind when viewing Figure C.1 through Figure C.8 in Appendix C that the SD and \%RSD values plotted in the figures are based only on replicate pairs. To precisely estimate SDs and \%RSD, much larger numbers of replicates than two are required. Hence, SDs and \%RSDs based on replicate pairs can be highly variable just by random chance. Therefore, a large SD or \%RSD may or may not be indicative of an outlier for one of two component concentration values in a replicate pair. Also, a \%RSD value can be large if the SD for a replicate pair is large relative to a small mean value.

Figure C.1 through Figure C.4 show no trends or dependence of SD or \%RSD values versus (i) pretransfer and five batch transfers or (ii) the size of the tank. Figure C.1(a) shows that all but one of the SD estimates for gibbsite are $\leq 0.10 \mathrm{lb} /$ gal approximately, with one higher value $\sim 0.26 \mathrm{lb} /$ gal. Figure C.2(a) shows that the SD estimates for SS are all $\leq 0.08$ and mostly $\leq 0.05 \mathrm{lb} /$ gal. Figure C.3(a) shows that the $\mathrm{SD}$ estimates for sand are all $\leq 0.25$ and mostly $\leq 0.15 \mathrm{lb} /$ gal. Figure C.4(a) shows that the SD estimates for $\mathrm{ZrO}_{2}$ are all $\leq 0.035$ and mostly $\leq 0.025$. In Figure C.1(b) through Figure C.4(b), the $\% \mathrm{RSD}$ values for some replicate pairs were quite large (i.e., 40-90). In several cases this appears to be a result of the low mass fraction of the component in the base simulant. Examples include (i) gibbsite for replicate Tests 6 and 7, and (ii) stainless steel for replicate Tests 16 and 17. In other cases, the large \%RSD values may be a result of an outlier for one of the two values. Examples include (i) gibbsite in pre-transfer samples from the 120-inch tank with replicate Tests 4 and 5, and (ii) $\mathrm{ZrO}_{2}$ in Batch 5 from the 120-inch tank with replicate Tests 6 and 7. However, as discussed previously, SD and \%RSD values estimated from two values may vary substantially. Finally, Figure C.1 through Figure C.4 do not show any dependence of SDs or \%RSDs on batches (pre-transfer and five batch transfers).

Figure C.5 through Figure C.8 plot SDs and \%RSDs versus the mean values from the replicate pairs, which provides for assessing whether testing variation is approximately constant on an absolute basis (i.e., SD) or relative basis (i.e., \%RSD). Figure C.5(a), Figure C.6(a), and Figure C.7(a) all suggest that the SD is larger for larger component concentrations, while Figure C.5(b), Figure C.6(b), and Figure C.7(b) suggest that \%RSDs do not have a consistent relationship with the mean values. Figure C.8(a) and Figure C.8(b) do not show any clear dependence of SD or \%RSD on mean concentrations of $\mathrm{ZrO}_{2}$. 


\subsection{Mixing and Transfer Performance}

Particle movement in, and transfer out, of stirred tanks is controlled by multiple physical phenomena related to particle-fluid interactions. Some examples of these physical phenomena include particle settling, settled particle mobilization (i.e., scouring), fluid jet decay and propagation, particle entrainment in the mixer jet and transfer pump suction, and turbulence effects on particle movement. For the SSMD scaled/system performance testing, as well as actual operations when WRPS transfers waste to WTP, the interest is in how well the compositions of pre-transfer samples from a tank represent the compositions of batch-transfer samples.

Section 4.1 discusses three possible measures for assessing mixing and transfer performance (MTP). Section 4.2 presents the results of investigating two of these measures using the test data described in Section 3.

\subsection{Possible Performance Measures}

For the SSMD system performance tests, there are several options for constructing performance measures using the concentrations (lb/gal) of base-simulant components (gibbsite, SS, sand, and $\mathrm{ZrO}_{2}$ ) in pre-transfer and batch-transfer samples. These include the

(a) difference of component concentrations (i.e., [batch transfer] - [pre-transfer])

(b) relative difference of component concentrations (i.e., [(batch transfer) - (pretransfer) $] /($ pre-transfer $)$ )

(c) ratio of component concentrations (i.e., batch transfer / pre-transfer) for each component.

Typically the choice between a difference, relative difference, and a ratio of pre-transfer versus posttransfer component values depends on whether the component concentrations have an additive error structure or a multiplicative error structure. In an additive error structure, the magnitude of a random error in a component value does not depend on the magnitude of the component value. In a multiplicative error structure, the magnitude of a random error in a component value does depend on the magnitude of the component value.

When the preceding options for a performance metric were investigated using test data, it was discovered that component concentrations in transfer batches were very similar to pre-transfer concentrations. Consequently, in the preceding options for performance measures, the effects of the test parameters tended to "cancel out." Hence, none of the three preceding options provided a desirable basis for modeling MTP as a function of the test parameters.

An alternative approach for assessing MTP is to use concentrations (lb/gal) of base-simulant components as performance measures and then model these performance measures as functions of the test parameters and the "batch" ( 0 and $1-5$, where 0 represents a pre-transfer sample). Then, if desired, the difference, relative difference, or ratio (as appropriate based on the error structure in the data) could be 
calculated based on the fitted models. As discussed in Section 4.2, the approach of modeling component concentrations was ultimately selected, where the forms of models considered are discussed in Section 5.

\subsection{Graphical Assessment of (Batch Transfer - Pre-Transfer) Performance Measures}

Figure 4.1 through Figure 4.4 display plots of concentration differences (Batch Transfer - PreTransfer) for each of the four $B S$ components, with the plot for the 43-inch tank on the left and the plot for the 120-inch tank on the right in each figure. Depending on the component, the traces (of concentration differences for the five batch transfers) are distributed within relatively narrow bands around zero.

- Figure 4.1 shows that most of the 26 test combinations have gibbsite concentration differences between -0.1 and $0.1 \mathrm{lb} /$ gal for both the 43 -inch and 120-inch tanks.

- Figure 4.2 shows that most of the 26 test combinations have SS concentration differences between (i) -0.05 and $0.05 \mathrm{lb} / \mathrm{gal}$ for the 43 -inch tank and (ii) -0.1 and $0.1 \mathrm{lb} /$ gal for the 120 -inch tank. Although 5 of the 26 test combinations for the 120-inch tank have the majority of their differences below $-0.1 \mathrm{lb} / \mathrm{gal}$, that is an insufficient basis on which to model SS concentration differences as a function of the test parameters.

- Figure 4.3 shows that most of the 26 test combinations have sand concentration differences between (i) -0.1 and $0.1 \mathrm{lb} /$ gal for the 43 -inch tank and (ii) -0.2 and $0.1 \mathrm{lb} /$ gal for the 120 -inch tank. Although 4 of the 26 test combinations for the 120-inch tank have the majority of their differences below $-0.2 \mathrm{lb} / \mathrm{gal}$, that is an insufficient basis on which to model sand concentration differences as a function of the test parameters.

- Figure 4.4 shows that most of the 26 test combinations have $\mathrm{ZrO}_{2}$ concentration differences between (i) -0.02 and $0.02 \mathrm{lb} /$ gal for both the 43-inch and 120-inch tanks. Although 6 of the 26 test combinations for the 120 -inch tank have the majority of their differences below $-0.02 \mathrm{lb} / \mathrm{gal}$ or above $0.02 \mathrm{lb} / \mathrm{gal}$, that is an insufficient basis on which to model $\mathrm{ZrO}_{2}$ concentration differences as a function of the test parameters.

Although the figures based on ratios (Option (c) in Section 4.1) are not included in this report, the conclusions were similar-namely, that the majority of the 26 test combinations had component concentration ratios fairly tightly grouped around 1.0. Preliminary modeling work using both differences and ratios confirmed what Figure 4.1 through Figure 4.4 suggest - namely, that narrow ranges of differences around 0 and ratios around 1 meant that the effects of the test parameters on component concentrations were "canceling out." For these reasons, it was decided to not model any of performance measures in Options (a), (b), or (c) in Section 4.1. Rather, it was decided to model component concentrations. 
43" Tank

$\stackrel{+}{i}$
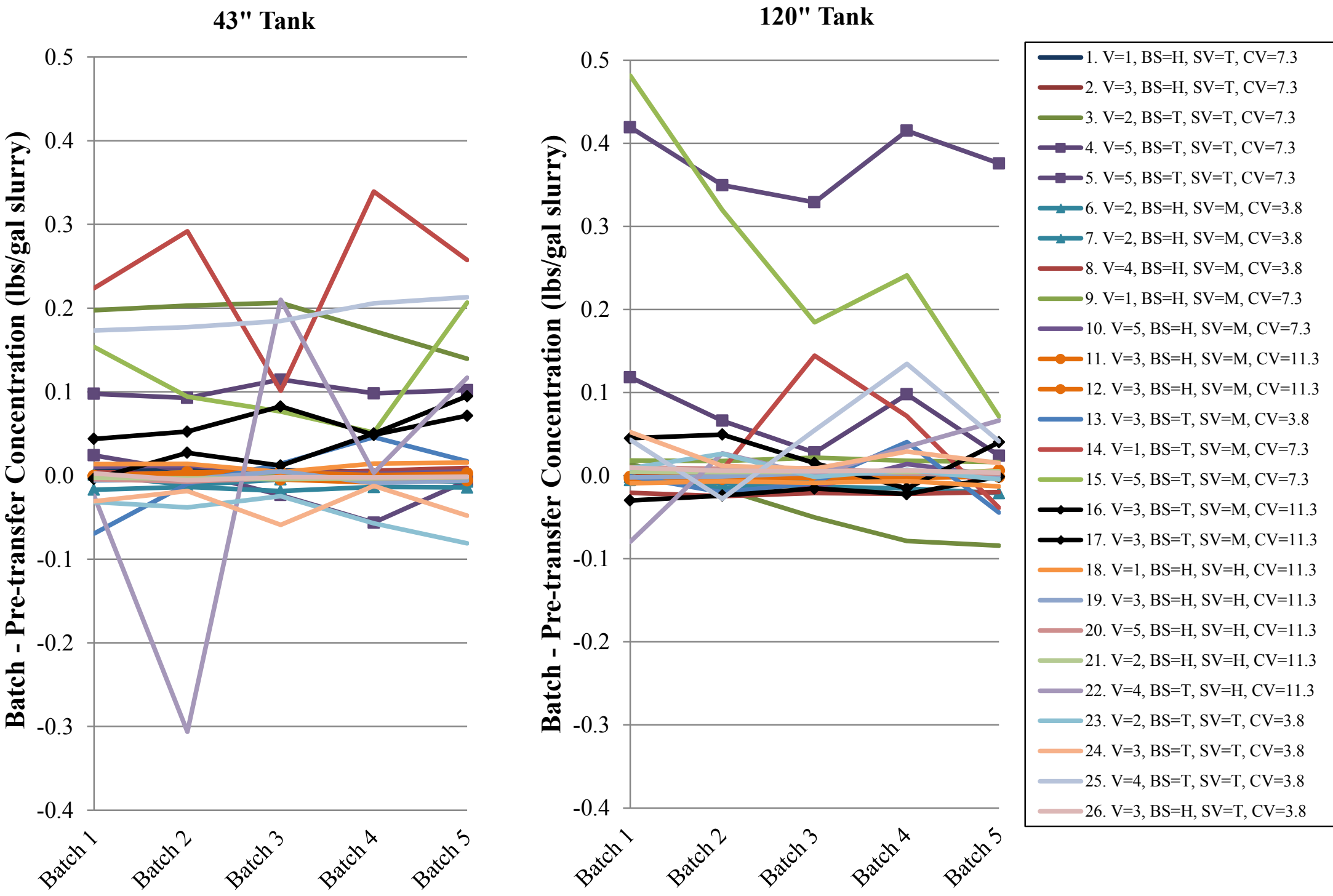

Figure 4.1. Gibbsite Concentration Differences (Batch Transfer - Pre-Transfer) for 26 Test Combinations Plotted versus Batch Transfer Number for 43-inch and 120-inch Tanks (For Information Only) 
43" Tank

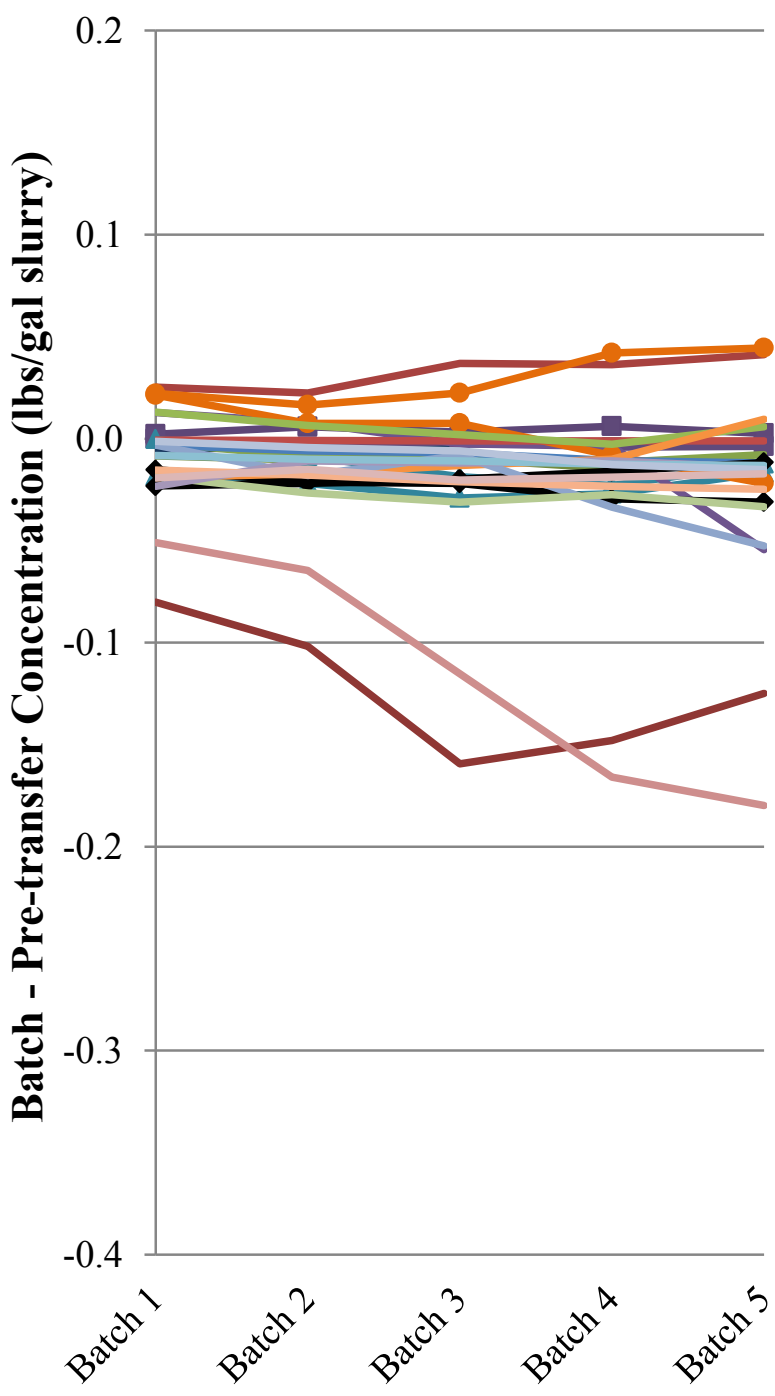

\section{0" Tank}

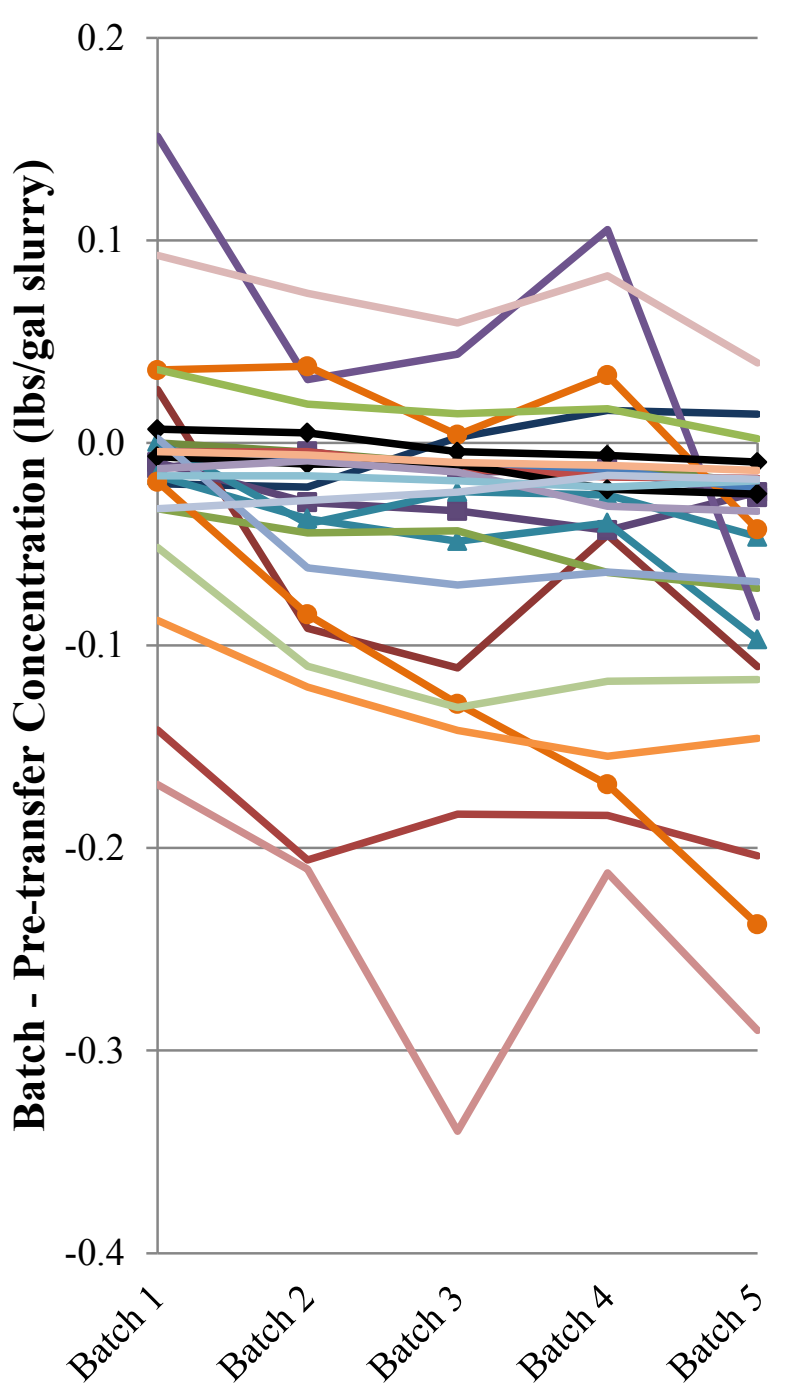

1. $\mathrm{V}=1, \mathrm{BS}=\mathrm{H}, \mathrm{SV}=\mathrm{T}, \mathrm{CV}=7.3$

2. $\mathrm{V}=3, \mathrm{BS}=\mathrm{H}, \mathrm{SV}=\mathrm{T}, \mathrm{CV}=7.3$

3. $\mathrm{V}=2, \mathrm{BS}=\mathrm{T}, \mathrm{SV}=\mathrm{T}, \mathrm{CV}=7.3$

-4. $\mathrm{V}=5, \mathrm{BS}=\mathrm{T}, \mathrm{SV}=\mathrm{T}, \mathrm{CV}=7.3$

-5. $\mathrm{V}=5, \mathrm{BS}=\mathrm{T}, \mathrm{SV}=\mathrm{T}, \mathrm{CV}=7.3$

-6. $\mathrm{V}=2, \mathrm{BS}=\mathrm{H}, \mathrm{SV}=\mathrm{M}, \mathrm{CV}=3.8$

-7. $\mathrm{V}=2, \mathrm{BS}=\mathrm{H}, \mathrm{SV}=\mathrm{M}, \mathrm{CV}=3.8$

8. $\mathrm{V}=4, \mathrm{BS}=\mathrm{H}, \mathrm{SV}=\mathrm{M}, \mathrm{CV}=3.8$

9. $\mathrm{V}=1, \mathrm{BS}=\mathrm{H}, \mathrm{SV}=\mathrm{M}, \mathrm{CV}=7.3$

10. $\mathrm{V}=5, \mathrm{BS}=\mathrm{H}, \mathrm{SV}=\mathrm{M}, \mathrm{CV}=7.3$

$\longrightarrow 11 . \mathrm{V}=3, \mathrm{BS}=\mathrm{H}, \mathrm{SV}=\mathrm{M}, \mathrm{CV}=11.3$

$-12 . \mathrm{V}=3, \mathrm{BS}=\mathrm{H}, \mathrm{SV}=\mathrm{M}, \mathrm{CV}=11.3$

13. $\mathrm{V}=3, \mathrm{BS}=\mathrm{T}, \mathrm{SV}=\mathrm{M}, \mathrm{CV}=3.8$

14. $\mathrm{V}=1, \mathrm{BS}=\mathrm{T}, \mathrm{SV}=\mathrm{M}, \mathrm{CV}=7.3$

15. $\mathrm{V}=5, \mathrm{BS}=\mathrm{T}, \mathrm{SV}=\mathrm{M}, \mathrm{CV}=7.3$

16. $\mathrm{V}=3, \mathrm{BS}=\mathrm{T}, \mathrm{SV}=\mathrm{M}, \mathrm{CV}=11.3$

-17. $\mathrm{V}=3, \mathrm{BS}=\mathrm{T}, \mathrm{SV}=\mathrm{M}, \mathrm{CV}=11.3$

18. $\mathrm{V}=1, \mathrm{BS}=\mathrm{H}, \mathrm{SV}=\mathrm{H}, \mathrm{CV}=11.3$

19. $\mathrm{V}=3, \mathrm{BS}=\mathrm{H}, \mathrm{SV}=\mathrm{H}, \mathrm{CV}=11.3$

20. $\mathrm{V}=5, \mathrm{BS}=\mathrm{H}, \mathrm{SV}=\mathrm{H}, \mathrm{CV}=11.3$

21. $\mathrm{V}=2, \mathrm{BS}=\mathrm{H}, \mathrm{SV}=\mathrm{H}, \mathrm{CV}=11.3$

22. $\mathrm{V}=4, \mathrm{BS}=\mathrm{T}, \mathrm{SV}=\mathrm{H}, \mathrm{CV}=11.3$

23. $\mathrm{V}=2, \mathrm{BS}=\mathrm{T}, \mathrm{SV}=\mathrm{T}, \mathrm{CV}=3.8$

24. $\mathrm{V}=3, \mathrm{BS}=\mathrm{T}, \mathrm{SV}=\mathrm{T}, \mathrm{CV}=3.8$

25. $\mathrm{V}=4, \mathrm{BS}=\mathrm{T}, \mathrm{SV}=\mathrm{T}, \mathrm{CV}=3.8$

26. $\mathrm{V}=3, \mathrm{BS}=\mathrm{H}, \mathrm{SV}=\mathrm{T}, \mathrm{CV}=3.8$

Figure 4.2. Stainless Steel Concentration Differences (Batch Transfer - Pre-Transfer) for 26 Test Combinations Plotted versus Batch Transfer Number for 43-inch and 120-inch Tanks (For Information Only) 


\section{3" Tank}

in

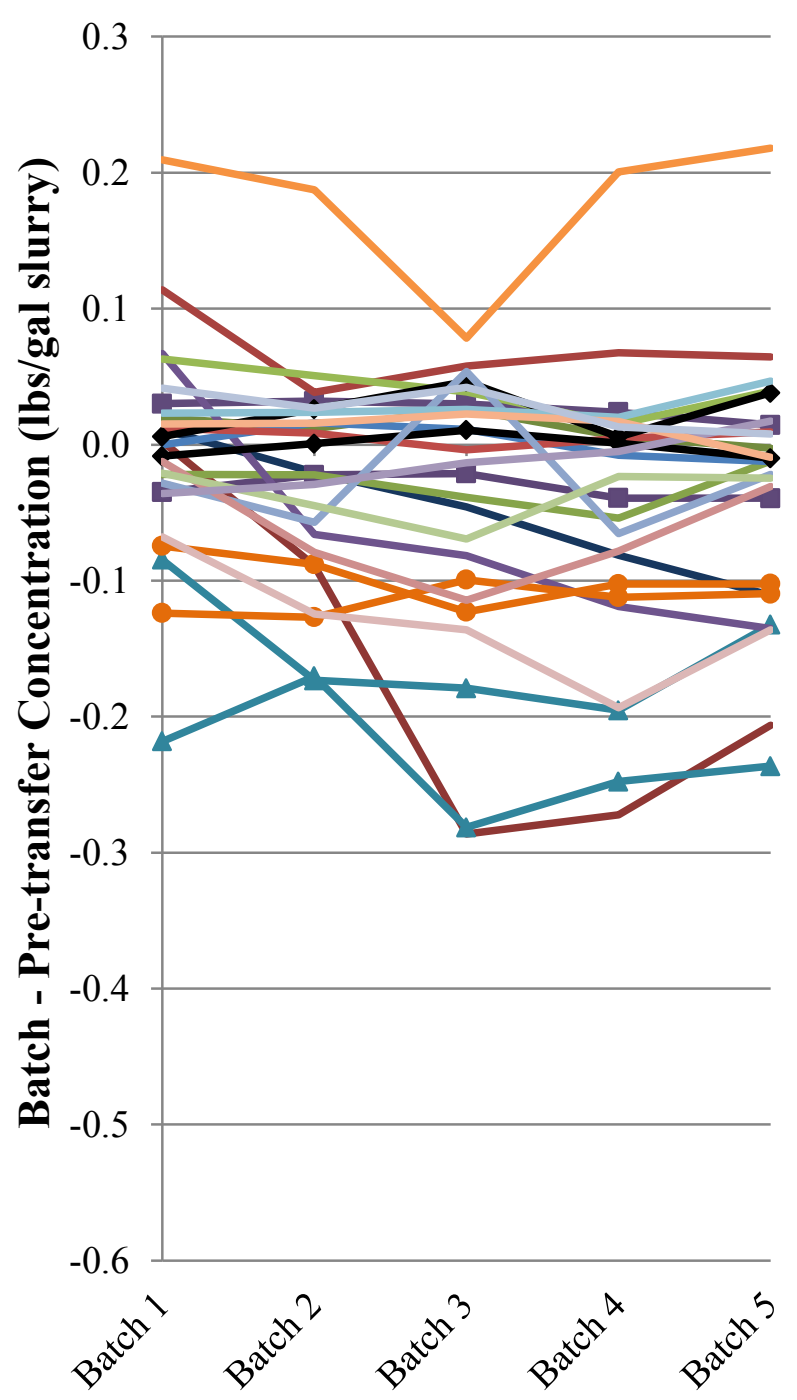

\section{0" Tank}

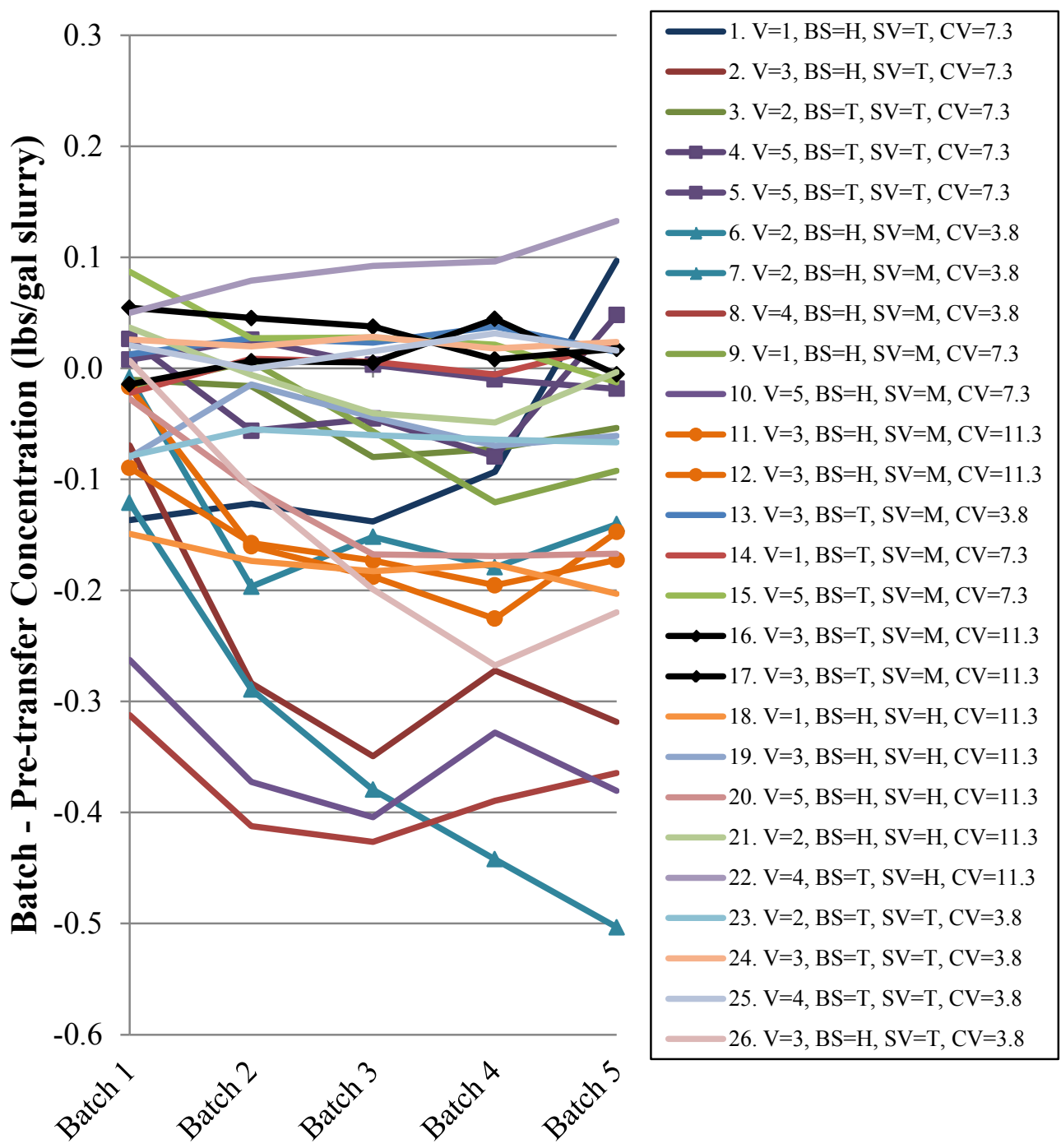

Figure 4.3. Sand Concentration Differences (Batch Transfer - Pre-Transfer) for 26 Test Combinations Plotted versus Batch Transfer Number for 43-inch and 120-inch Tanks (For Information Only) 


\section{3" Tank}

$\dot{a}$

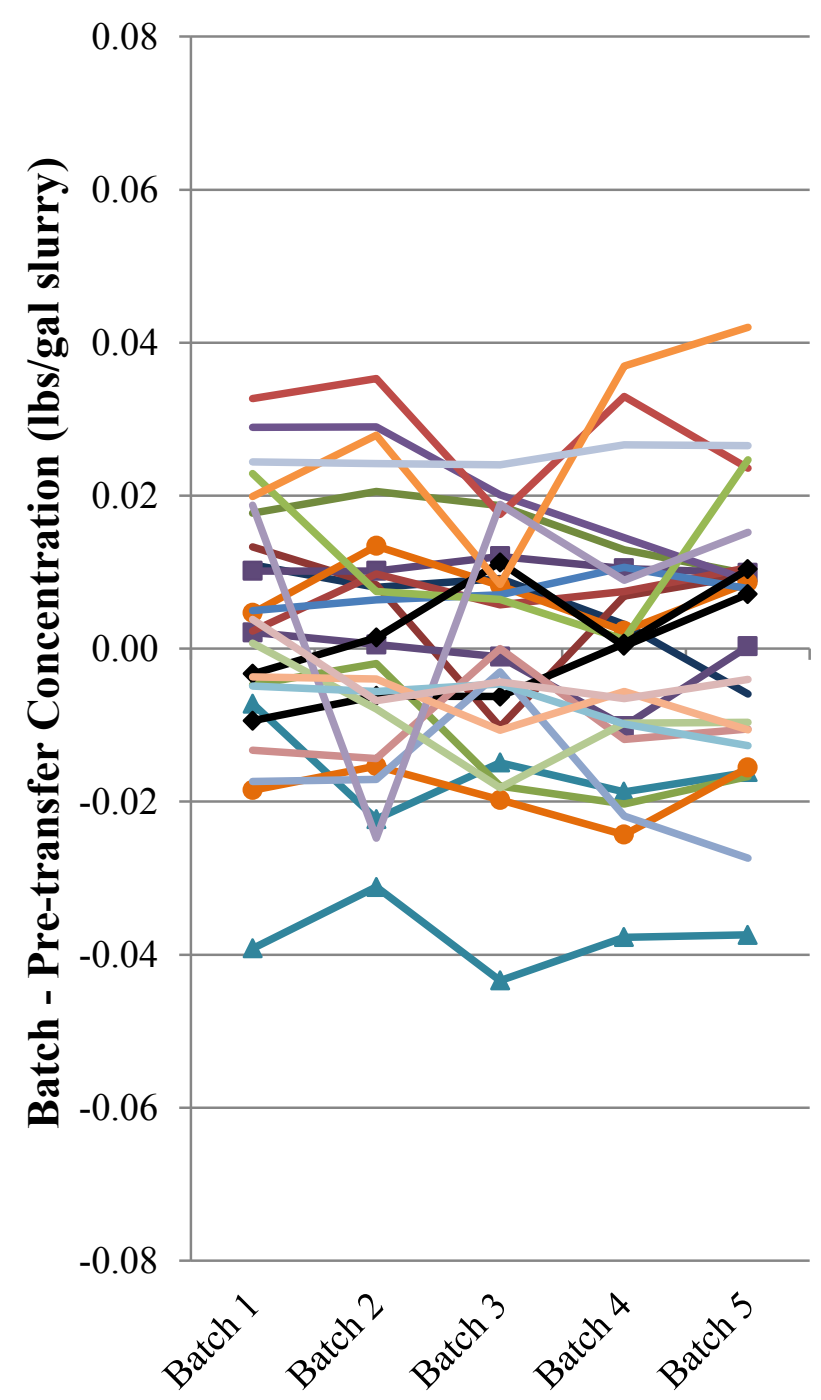

120" Tank

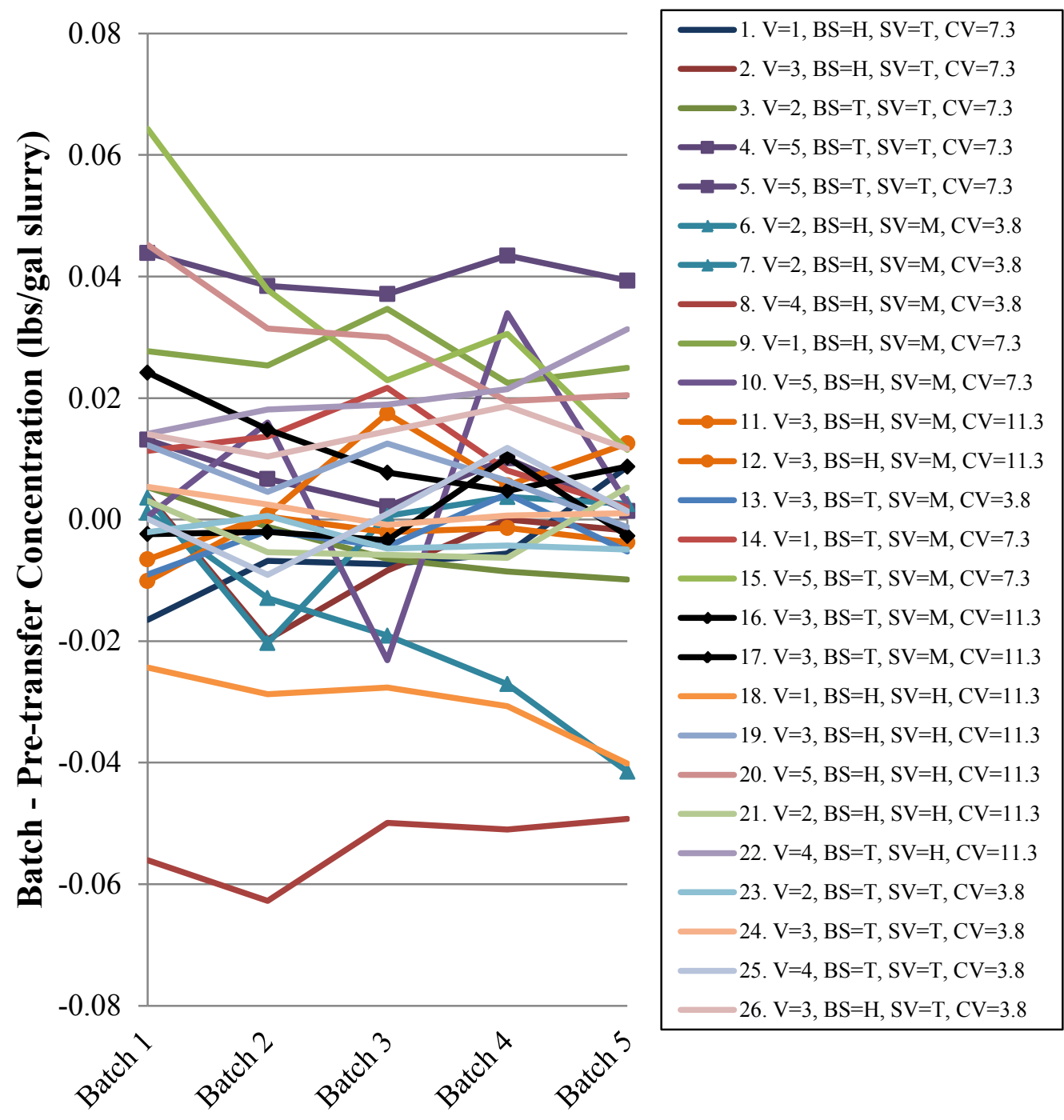

Figure 4.4. $\mathrm{ZrO}_{2}$ Concentration Differences (Batch Transfer - Pre-Transfer) for 26 Test Combinations Plotted versus Batch Transfer Number for 43-inch and 120-inch Tanks (For Information Only) 


\subsection{Models for Component Concentrations as Functions of Test Parameters}

One of the steps for developing a scaling relationship based on the SSMD performance tests is to develop models that predict performance for each of the two scaled tanks as functions of the test parameters varied in the testing (see Step 2 of Section 1). As noted in Section 4.2, preliminary data assessments and modeling led to the decision to develop models that predict the concentrations of basesimulant components.

For specific mixing phenomena (e.g., critical suspension velocity or effective cleaning radius), theoretical or physics-based models have been developed to relate a given phenomenon to specific parameters of interest (e.g., mixer-jet nozzle velocity). These models generally have the advantage that they implicitly incorporate geometric scaling, and thus allow for better extrapolative predictions related to scale. However, the difficulty encountered in batch transfer testing is that the characteristic of primary interest (i.e., the composition of a batch-transfer sample relative to the composition of a pre-transfer sample) is a complicated result of interactions between the mixing phenomena, particle settling phenomena, and material transfer phenomena. Although mixing, settling, and transfer phenomena may be reasonably well understood individually, less is known about the interactions of these phenomena, which result in changes to the mixed volume and composition of the tank. Even less is known about relating compositions of pre-transfer samples and batch-transfer samples. For these reasons, an empirical approach to developing models was chosen for this work. Polynomial models (i.e., Taylor-series expansions) can adequately approximate many functions, have terms that represent individual and interactive effects of test parameters, and have been successfully used for many decades in various fields of application when theoretical or physics-based models are not available.

Sections 5.1 and 5.2 discuss two empirical model forms that relate the natural logarithms of component (gibbsite, SS, sand, and $\mathrm{ZrO}_{2}$ ) concentrations to the SSMD performance test parameters: mixer-jet nozzle velocity $(U)$, base simulant $(B S)$, supernatant viscosity $(S V),{ }^{(a)}$ transfer-line capture velocity $(C V)$, and batch number $(B N)$. The $B N$ parameter takes a value of 0 for pre-transfer samples and values of 1 to 5 for the batch-transfer samples. Both model forms discussed apply to a single tank, either the 43 -inch tank or the 120-inch tank. Section 5.3 discusses an empirical model form that is an extension of the model in Section 5.1, which includes a scaling equation and hence would be fit to combined data from both scaled tanks. The modeling approach in Section 5.3 is the main one envisioned by Greer (2012) and Lee et al. (2012a), but the assumptions associated with that model form do not hold (see Section 7). Hence, the models in Sections 5.1 and 5.2, along with the EP approach discussed in Section 6, are the primary basis for developing scaling relationships in this report.

The natural logarithm of concentrations of the $j^{\text {th }}$ component $\left[\ln \left(C_{j}\right)\right]$ are modeled for three reasons. First, ordinary least squares (OLS) regression methods assume that all response variable values have a common experimental and measurement variance. Because the $C_{j}$ values for some components " $j$ " range from very small values to values near (or more than) an order of magnitude larger, it is more likely that $\ln \left(C_{j}\right)$ values rather than $C_{j}$ values satisfy the OLS assumption. Second, it was noticed in Section 3.3.1 that $\ln \left(C_{j}\right)$ and $B N$ are generally linearly related for all four components. Third, one of the model forms

(a) As discussed subsequently, it was decided to use kinematic viscosity (KV) in models rather than SV. 
considered in this section is based on a linearization and extension of a power-law relationship between $C_{j}$ and $U$, which corresponds to a linear relationship between $\ln \left(C_{j}\right)$ and $\ln (U)$.

The empirical approach to modeling does have limitations with respect to extrapolation and prediction, but there were no theoretical or physics-based model alternatives at the time of this work. As discussed in Section 1, scope for the work in this report was to develop scaling relationships that are applicable between the two scales tested. Subsequent work, to be performed and documented separately by different researchers, will consider the options for extending the work of this report to full-scale DSTs.

\subsection{Models for Component Concentrations as Functions of the Test Parameters with a Cubic Polynomial Dependence on Jet Velocity}

This section presents and discusses polynomial model forms for the natural logarithms of component concentration in pre-transfer or batch-transfer samples as a function of the test parameters varied in the SSMD performance testing $(U, \overline{B S}, \overline{K V}, \overline{C V}$, and $B N)$. These model forms are based on the assumption that a cubic polynomial is adequate to approximate the dependence of component concentrations on $U$.

Section 5.1.1 presents a polynomial model form for the natural log of component concentrations for each component, tank, and $B N$. Section 5.1.2 presents an extension of this polynomial model that includes terms involving $B N$.

\subsubsection{Polynomial Model Form for Component Concentrations for Each Tank and Pre-Transfer or Batch Transfer}

One of the polynomial model forms used to relate component concentrations to SSMD test parameters is similar to the one listed in Equation (2.3), which was specified by Greer (2012) for the purpose of developing the experimental design in Table 2.3. There are three changes to the model form of Equation (2.3) used to obtain the model form discussed in this subsection:

1. The model predicts the natural logarithm of concentration of the $i^{\text {th }}$ component $\left(C_{i}\right)$ rather than a performance measure that compares component concentrations from batch-transfer samples and pre-transfer samples.

2. The kinematic viscosity $(K V)$, which is the fluid viscosity divided by the fluid density, is used in place of the dynamic viscosity of the supernatant $(S V)$.

3. Coded values of all test parameters except $U$ are used to address high collinearity among model terms.

The reasons for these changes are discussed in the following paragraphs.

When the experiment discussed in Section 2.2 was designed, it was envisioned that the supernatants would be developed so that the supernatant density would have a linear relationship with $S V$. In that case, it was decided to use $S V$ to represent the joint effects of supernatant viscosity and density. However, ultimately it was not possible to obtain supernatants with densities that had the desired linear relationship with viscosities. Also, as noted in Section 2.1, the linear relationship is actually between $\ln (S V)$ and supernatant density. Figure 5.1 shows the actual supernatant densities plotted versus the natural 
logarithm of actual supernatant dynamic viscosities for the tests in Table 2.3 and Table 2.4. The figure does not show a linear relationship.

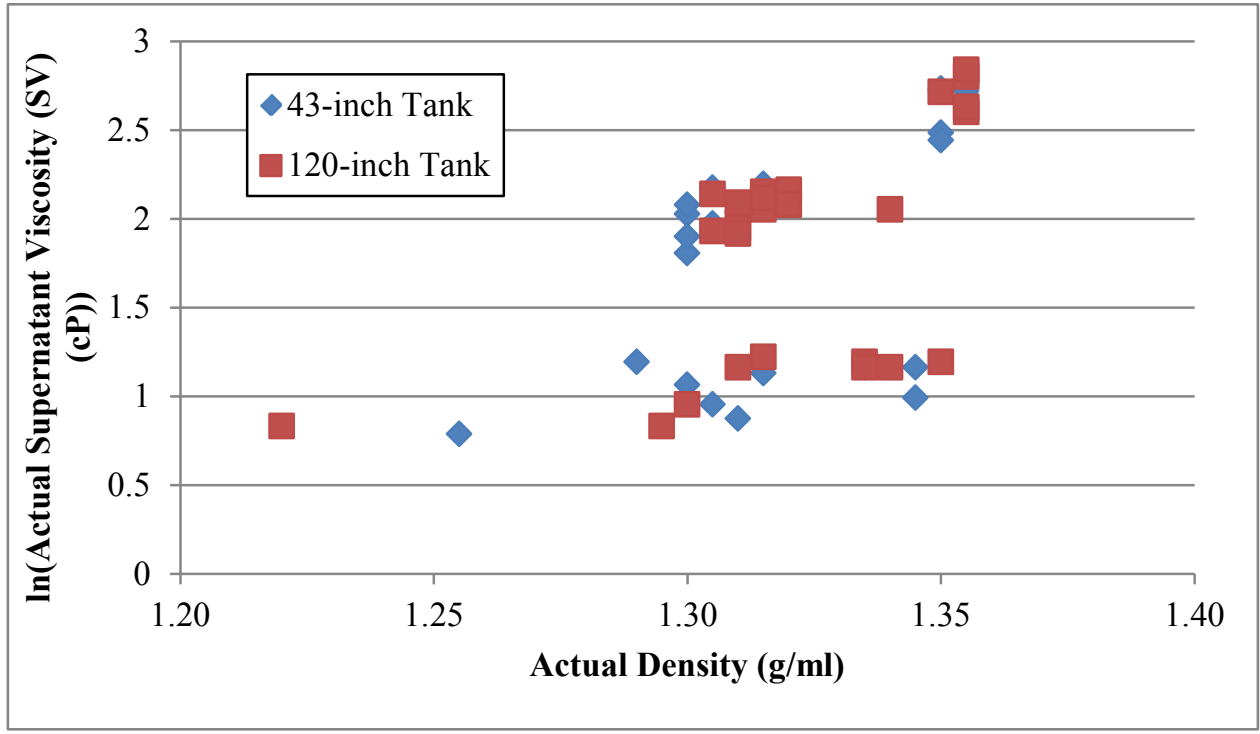

Figure 5.1. Plot of the Natural Logarithm of Actual Dynamic Viscosity of Supernatants $[\ln (S V)]$ versus Actual Bottom Density for the 22 Test Combinations in Table 2.3 and the 4 Additional Test Combinations in Table 2.4 (For Information Only)

It was decided to use $K V$ instead of $S V$ in the model because $K V$ is commonly used for modeling the behavior of fluids. However, in this case it makes no practical difference because $K V$ is highly linearly related to $S V$, as shown in Figure 5.2.

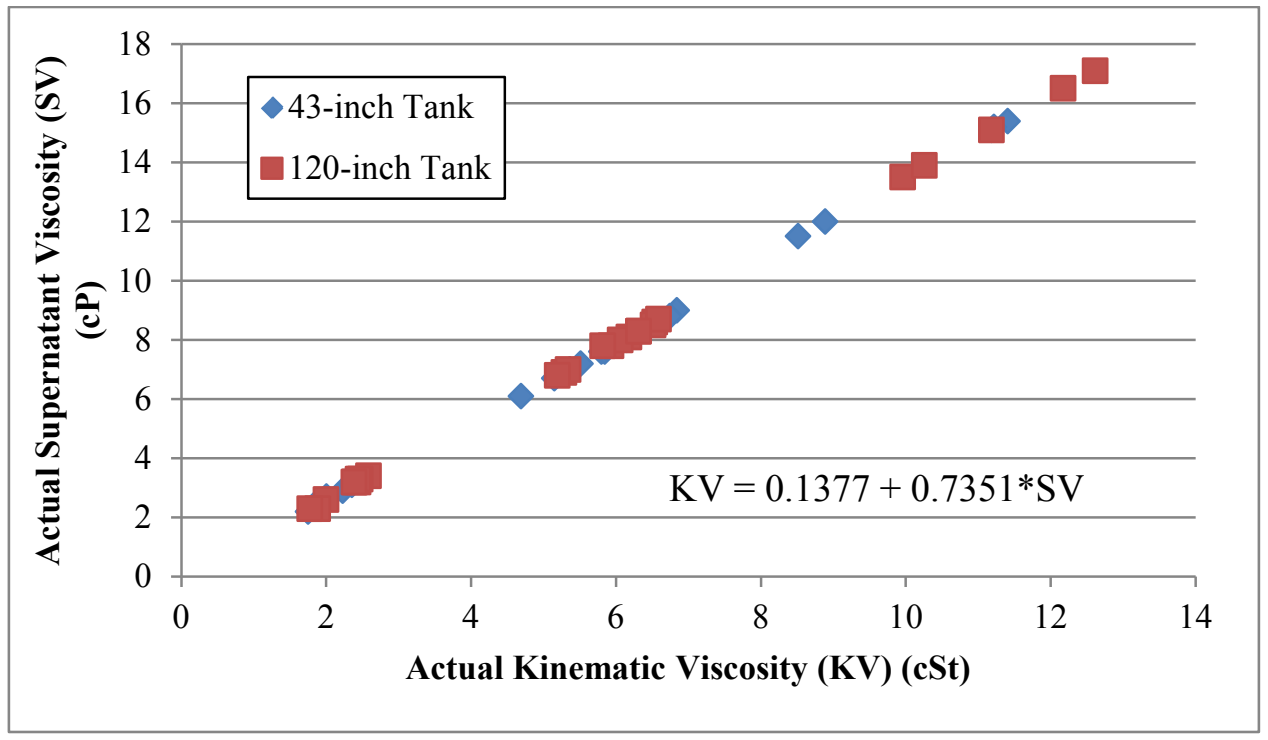

Figure 5.2. Plot of Kinematic Viscosity $(K V)$ versus Dynamic Viscosity $(S V)$ for the Supernatants of the 22 Test Combinations in Table 2.3 and the 4 Additional Test Combinations in Table 2.4 (For Information Only) 
With the three changes to Equation (2.3) described above, the new model becomes

$$
\begin{aligned}
\ln \left(C_{i j k}\right) & =b_{0}^{i j k}+b_{1}^{i j k} U_{i}+b_{2}^{i j k} U_{i}^{2}+b_{3}^{i j k} U_{i}^{3}+b_{4}^{i j k} \overline{B S}+b_{5}^{i j k} \overline{K V}+b_{6}^{i j k} \overline{K V}^{2}+b_{7}^{i j k} \overline{C V} \\
& +b_{8}^{i j k}\left[\overline{B S} * U_{i}\right]+b_{9}^{i j k}\left[\overline{B S} * U_{i}^{2}\right]+b_{10}^{i j k}[\overline{B S} * \overline{K V}]+b_{11}^{i j k}\left[\overline{B S} * \overline{K V}^{2}\right] \\
& +b_{12}^{i j k}\left[\overline{K V} * U_{i}\right]+b_{13}^{i j k}\left[\overline{K V}^{2} * U_{i}\right]+b_{14}^{i j k}\left[\overline{K V} * U_{i}^{2}\right]+b_{15}^{i j k}\left[\overline{K V}^{2} * U_{i}^{2}\right]+\varepsilon_{i j k}
\end{aligned}
$$

where $\quad C_{i j k}=$ concentration $(\mathrm{lb} / \mathrm{gal})$ of the $j^{\text {th }}$ base-simulant component in the $k^{\text {th }}$ batch transfer $(k=0,1,2,3,4,5)$ from the $i^{\text {th }} \operatorname{tank}(i=1,2)$

$U_{i}=$ mixer-jet nozzle velocity $(\mathrm{ft} / \mathrm{sec})$ in the $i^{\text {th }}$ tank, where " $i$ " $=43$ or 120

$\overline{B S}=$ base simulant, coded so that Typical $=-1$ and High $=+1$.

$\overline{K V}=$ kinematic viscosity, coded according to Equation (5.2) so that the smallest and largest $K V$ values (over both size tanks) are coded to -1 and +1 , respectively

$\overline{C V}=$ capture velocity, coded according to Equation (5.3) so that the smallest and largest $C V$ values (over both size tanks) are coded to -1 and +1 , respectively

$\varepsilon_{i j k}=$ random error in the concentration of the $j^{\text {th }}$ base-simulant component in the $k^{\text {th }}$ batch transfer $(k=0,1,2,3,4,5)$ from the $i^{\text {th }} \operatorname{tank}(i=1,2)$. These random errors are assumed to be independently and identically distributed according to the normal (Gaussian) distribution with mean 0 and variance $\sigma^{2}$.

$b_{0}^{i j k}$ to $b_{15}^{i j k}=$ model coefficients for the $j^{\text {th }}$ base-simulant component in the $k^{\text {th }}$ batch transfer from the $i^{\text {th }}$ tank.

Several aspects of this model require explanation:

1. Note that $k=0$ corresponds to pre-transfer samples, whereas $k=1,2, \ldots, 5$ correspond to the five batch-transfer samples.

2. The bars over $B S, K V$, and $C V$ denote coded values. The formulas for the coding of $K V$ and $C V$ are given subsequently. The test parameter $U$ is not coded for consistency with the model form discussed in Section 5.2, which uses $\ln (U)$ terms with $U$ uncoded. For a model linear in the coefficients as given in Equation (5.1), a model fitted with coded values of parameters gives exactly the same model fit that would be obtained without coding the parameters. This is not to say that the model coefficients will be exactly the same, but rather that the variation in the response variable accounted for by the model (i.e., $\mathrm{R}^{2}$ ) will be the same.

3. The assumption that the $\varepsilon_{i j k}$ are independently and identically distributed according to the normal (Gaussian) distribution with mean 0 and variance $\sigma^{2}$ is required to use OLS regression to fit the model to data and to quantify the uncertainties of model predictions and the uncertainties of estimated model coefficients. This assumption is judged more appropriate for 
$\ln \left(C_{i j k}\right)$ than for $C_{i j k}$, which is one of the three reasons for modeling $\ln \left(C_{i j k}\right)$ discussed in Section 5.0 .

The qualitative values of $B S$ (typical, high) were coded as numeric values $(-1,+1)$ to enable using OLS regression methods. The test parameters $K V$ and $C V$ were also coded so that the smallest and largest values (over both size tanks) are coded to -1 and +1 , respectively. The general formula for the coded values of these parameters $(\bar{P})$ is given by

$$
\bar{P}=\frac{P-\left(\frac{\max (P)+\min (P)}{2}\right)}{\left(\frac{\max (P)-\min (P)}{2}\right)}
$$

where $P$ denotes a parameter, and "min" and "max" denote the minimum and maximum values of the parameter. The minimum and maximum values were determined over all batches (pre-transfer and five transfer batches) and over both tank sizes. The specific formulas for the coded values of $K V$ and $C V$ are given by

$$
\overline{K V}=\frac{K V-7.186}{5.433}
$$

and

$$
\overline{C V}=\frac{C V-7.479}{3.778}
$$

The values presented in Equations (5.3) and (5.4) are approximate because the values were rounded to three decimal places.

This type of coding helps reduce the collinearity among terms in the model compared to using uncoded values of $K V$ and $C V$ in Equation (5.1). Collinearity means that there is a near linear relationship among a set of model terms. The consequences of strong collinearity among terms in a regression model are that (i) not all of those terms can be included in the model at the same time, and (ii) when using stepwise regression (see Section 5.4) to select significant model terms, the wrong model terms can be selected.

Equation (5.1) includes (i) an intercept, (ii) terms for the linear, quadratic, and cubic effects of $U$, (iii) the effect of $B S$, (iv) linear and quadratic effects for $K V$, and (v) a linear effect of $C V$. In addition, the model contains terms for two-parameter interaction effects that were considered by Greer (2012) and Lee et al. (2012a) to be of interest or potentially important. These include the interactions $\overline{B S} * U, \overline{B S} * U^{2}$, $\overline{B S} * \overline{K V}, \overline{B S} * \overline{K V}^{2}, \overline{K V} * U, \overline{K V}^{2} * U, \overline{K V} * U^{2}$, and $\overline{K V}^{2} * U^{2}$. Also, because the effect of $C V$ was expected to be small based on previous testing (Adamson et al. 2009; Lee 2012), $C V$ was not expected to have a large interaction effect with any of the other parameters. Hence, no interaction effects with $C V$ are included in the model in Equation (5.1). 
The model in Equation (5.1) has the same form for each tank, each base-simulant component, and each $B N(k=0,1,2,3,4,5)$, but the model coefficients ( $b_{0}^{i j k}$ to $b_{15}^{i j k}$ ) may change for the two tanks, the base-simulant components $(j)$, and the $B N$. The coefficients $b_{0}^{i j k}$ to $b_{15}^{i j k}$ could also be estimated by fitting the model to the experimental data from the $i^{\text {th }}$ tank for each base-simulant component and each $B N$.

\subsubsection{Models for Component Concentrations as Functions of the Test Parameters with a Cubic Polynomial Dependence on Jet Velocity}

During preliminary data modeling work, stepwise regression (see Section 5.4) was used to select significant terms from among all terms in Equation (5.1), which was done separately using the data for each component, tank, and $B N$. The terms in the models for different $B N$ s were generally similar, but not exactly the same. The differences were attributed to the variation in the data across the $B N \mathrm{~s}$, as seen in Figure B.1 to Figure B.8 in Appendix B.

Previous testing (Jensen 2011) suggests that there may be a trend in some component concentrations from pre-transfer and through the batch transfers, which would be useful to model along with the effects of other test parameters. Because the relationships of natural logarithms of component concentrations to $B N$ (see Figure B.5 to Figure B.8) were generally linear (as noted in Section 3.3), the model of Equation (5.1) was extended to include terms associated with $B N$ effects. The relationships in Figure B.5 to Figure B. 8 indicate that there may be differences in intercepts and slopes of the linear relationships of $\ln \left(C_{i j k}\right)$ with $B N$ depending on the test, so the model in Equation (5.1) is extended to allow the other test parameters to affect both the intercept and slope of the linear relationships. The extension of the model in Equation (5.1) is given by

$$
\begin{aligned}
\ln \left(C_{i j}\right) & =b_{0}^{i j}+b_{1}^{i j} U_{i}+b_{2}^{i j} U_{i}^{2}+b_{3}^{i j} U_{i}^{3}+b_{4}^{i j} \overline{B S}+b_{5}^{i j} \overline{K V}+b_{6}^{i j} \overline{K V}^{2}+b_{7}^{i j} \overline{C V} \\
& +b_{8}^{i j}\left[\overline{B S} * U_{i}\right]+b_{9}^{i j}\left[\overline{B S} * U_{i}^{2}\right]+b_{10}^{i j}[\overline{B S} * \overline{K V}]+b_{11}^{i j}\left[\overline{B S} * \overline{K V}^{2}\right] \\
& +b_{12}^{i j}\left[\overline{K V} * U_{i}\right]+b_{13}^{i j}\left[\overline{K V}^{2} * U_{i}\right]+b_{14}^{i j}\left[\overline{K V} * U_{i}^{2}\right]+b_{15}^{i j}\left[\overline{K V}^{2} * U_{i}^{2}\right] \\
& +b_{16}^{i j} B N+b_{17}^{i j} B N^{2}+b_{18}^{i j}\left[B N * U_{i}\right]+b_{19}^{i j}[B N * \overline{B S}]+b_{20}^{i j}[B N * \overline{K V}] \\
& +b_{21}^{i j}[B N * \overline{C V}]+b_{22}^{i j}\left[B N * \overline{B S} * U_{i}\right]+b_{23}^{i j}[B N * \overline{B S} * \overline{K V}] \\
& +b_{24}^{i j}\left[B N * \overline{K V} * U_{i}\right]+\varepsilon_{i j}
\end{aligned}
$$

where $\quad C_{i j}=$ concentration $(\mathrm{lb} / \mathrm{gal})$ of the $j^{\text {th }}$ base-simulant component in the $i^{\text {th }} \operatorname{tank}(i=1,2)$

$U_{i}=$ mixer-jet nozzle velocity in the $i^{\text {th }} \operatorname{tank}(\mathrm{ft} / \mathrm{sec})$

$\overline{B S}=$ base simulant, coded so that Typical $=-1$ and High $=+1$.

$\overline{K V}=$ kinematic viscosity, coded according to Equation (5.3) so that the smallest and largest $K V$ values (over both size tanks) are coded to -1 and +1 , respectively 


$$
\begin{aligned}
\overline{C V}= & \text { capture velocity, coded according to Equation (5.4) so that the smallest and largest } \\
& C V \text { values (over both size tanks) are coded to }-1 \text { and }+1, \text { respectively } \\
B N= & \text { batch number }(k=0,1, \ldots, 5) \text { where } k=0 \text { denotes pre-transfer, and } k=1,2, \ldots, 5 \\
& \text { denote transfer batches } 1,2, \ldots, 5 . \\
\varepsilon_{i j}= & \text { random error in the concentration of the } j^{\text {th }} \text { base-simulant component in the } i^{\text {th }} \text { tank } \\
& (i=1,2) . \text { These random errors are assumed to be independently and identically } \\
& \text { distributed according to the normal (Gaussian) distribution with mean } 0 \text { and } \\
& \text { variance } \sigma^{2} . \\
b_{0}^{i j} \text { to } b_{24}^{i j}= & \text { model coefficients for the } j^{\text {th }} \text { base-simulant component in the } i^{\text {th }} \text { tank. }
\end{aligned}
$$

Some of the notation in Equation (5.5) was defined previously, but for completeness, all notation is defined above. The following observations are made regarding the model in Equation (5.5):

1. The portion of the model with coefficients $b_{0}^{i j}$ to $b_{15}^{i j}$ (i.e., terms that do not involve $B N$ ) predicts $\ln \left(C_{i j}\right)$ for pre-transfer samples, because that portion of the model remains when $B N=0$.

2. The model contains nine terms involving $B N$ (with coefficients $b_{16}^{i j}$ to $b_{24}^{i j}$ ). These terms involve (i) a linear effect of $B N$, (ii) a quadratic effect of $B N$ (i.e., $B N^{2}$ ), (iii) the two-parameter interactions $B N * U_{i}, B N * \overline{B S}, B N * \overline{K V}$, and $B N * \overline{C V}$, and (iv) the three-parameter interactions $B N * \overline{B S} * U_{i}, B N * \overline{B S} * \overline{K V}$, and $B N * \overline{K V} * U_{i}$.

3. The coefficients $b_{0}^{i j}$ to $b_{24}^{i j}$ depend only on the tank $(i)$ and the base-simulant component $(j)$ and not on the batch-transfer number $(k)$, as is the case in Equation (5.1). This is because the model in Equation (5.5) assumes that all of the effects of pre-transfer and the five batch transfers are represented by the terms with coefficients $b_{16}^{i j}$ to $b_{24}^{i j}$.

4. In the statistics literature when using empirical models to adequately approximate the true, unknown relationship between the response variable and predictor parameters, three-parameter interactions such as the terms $B N * \overline{B S} * U_{i}, B N * \overline{B S} * \overline{K V}$, and $B N * \overline{K V} * U_{i}$ in Equation (5.5) are typically assumed to be negligible and are not included in the model. However, it is not clear whether only two-parameter interactions involving $B N$ will be sufficient to account for differences in $\ln \left(C_{i j}\right)$ vs. $B N$ relationships over the different test combinations. So, it was decided to consider such model terms, and rely on stepwise regression (see Section 5.4) to determine whether such terms are needed in the model. 


\subsection{Models for Component Concentrations as Functions of the Test Parameters with a Power Law Dependence on Jet Velocity}

The model form discussed in Section 5.1 is based on the assumption that the relationship between $\ln \left(C_{i j}\right)$ and $U$ can be adequately approximated by a cubic polynomial in $U$. The model form in this section is based on the assumption that the relationship between $C_{i j}$ and $U$ follows a power-law model

$$
C_{i j}=d_{0} U^{d_{1}}
$$

or equivalently that the relationship between $\ln \left(C_{i j}\right)$ and $\ln (U)$ is linear

$$
\ln \left(C_{i j}\right)=\ln \left(d_{0}\right)+d_{1} \ln (U)
$$

The model form in this section was extended to allow for up to a quadratic relationship between $\ln \left(C_{i j}\right)$ and $\ln (U)$, although that goes beyond a linearized form of the traditional power-law model (which only depends on $\ln (U))$.

The following model was developed by replacing the terms in Equation (5.5) involving $U_{i}$ and $U_{i}^{2}$ with terms involving $\ln \left(U_{i}\right)$ and $\left[\ln \left(U_{i}\right)\right]^{2}$, respectively, and dropping the term involving $U_{i}^{3}$ :

$$
\begin{aligned}
\ln \left(C_{i j}\right) & =c_{0}^{i j}+c_{1}^{i j} \ln \left(U_{i}\right)+c_{2}^{i j}\left[\ln \left(U_{i}\right)\right]^{2}+c_{3}^{i j} \overline{B S}+c_{4}^{i j} \overline{K V}+c_{5}^{i j} \overline{K V}^{2}+c_{6}^{i j} \overline{C V} \\
& +c_{7}^{i j}\left[\overline{B S} * \ln \left(U_{i}\right)\right]+c_{8}^{i j}\left[\overline{B S} *\left[\ln \left(U_{i}\right)\right]^{2}\right]+c_{9}^{i j}[\overline{B S} * \overline{K V}]+c_{10}^{i j}\left[\overline{B S} * \overline{K V}^{2}\right] \\
& +c_{11}^{i j}\left[\overline{K V} * \ln \left(U_{i}\right)\right]+c_{12}^{i j}\left[\overline{K V}^{2} * \ln \left(U_{i}\right)\right]+c_{13}^{i j}\left[\overline{K V} *\left[\ln \left(U_{i}\right)\right]^{2}\right] \\
& +c_{14}^{i j}\left[\overline{K V}{ }^{2} *\left[\ln \left(U_{i}\right)\right]^{2}\right]+c_{15}^{i j} B N+c_{16}^{i j} B N^{2}+c_{17}^{i j}\left[B N * \ln \left(U_{i}\right)\right] \\
& +c_{18}^{i j}[B N * \overline{B S}]+c_{19}^{i j}[B N * \overline{K V}]+c_{20}^{i j}[B N * \overline{C V}]+c_{21}^{i j}\left[B N * \overline{B S} * \ln \left(U_{i}\right)\right] \\
& +c_{22}^{i j}[B N * \overline{B S} * \overline{K V}]+c_{23}^{i j}\left[B N * \overline{K V} * \ln \left(U_{i}\right)\right]+\varepsilon_{i j}
\end{aligned}
$$

where all notation is the same as defined after Equation (5.5), except that the model coefficients are denoted by $c_{0}^{i j}$ to $c_{23}^{i j}$. Hence, this model has one less term compared to the model in Equation (5.5). Also, as was the case for the model in Equation (5.5), this model includes 3 three-parameter interaction terms involving $B N$, even though typically three-parameter interactions would not be included (see the discussion in Item 4 at the end of Section 5.1.2). Because stepwise regression (see Section 5.4) was used to select only those model terms that are statistically significant, if the three-parameter interaction terms were not significant, they were not selected.

If any of the terms involving $\left[\ln \left(U_{i}\right)\right]^{2}$ are statistically significant when stepwise regression is applied to the data, it would indicate that there is curvature in the relationship between $\ln \left(C_{i j}\right)$ and $\ln \left(U_{i}\right)$. In turn, 
this would mean that the assumption of a power-law relationship between $C_{i j}$ and $U_{i}$ is not consistent with the test data.

\subsection{Models for Component Concentrations as Functions of the Test Parameters and Tank Diameter}

The models in Equation (5.5) of Section 5.1 and in Equation (5.8) of Section 5.2 are for component concentrations in an individual scaled tank. Greer (2012) and Lee et al. (2012a) envisioned combining a model applicable for each tank with a simple, geometric-scaling equation provided in Lee (2012):

$$
U_{2}=U_{i}\left(\frac{D_{2}}{D_{i}}\right)^{a}
$$

where $D_{i}$ represents the diameter of the $i^{\text {th }} \operatorname{tank}(i=1,2), U_{i}$ represents the mixer-jet nozzle velocity of the $i^{\text {th }}$ tank, and " $a$ " represents the scaling exponent. This simple scaling equation provides for calculating the velocity $U_{2}$ in a tank of diameter $D_{2}$ that yields EP as the velocity $U_{1}$ in a tank of diameter $D_{1}$, given the appropriate scaling exponent $(a)$. In this report $U_{1}$ and $D_{1}$ are denoted $U_{43}$ and $D_{43}$, respectively, while $U_{2}$ and $D_{2}$ are denoted $U_{120}$ and $D_{120}$, respectively.

Equation (5.9) could be substituted into either Equation (5.5) or Equation (5.8) to yield a model that could be fit (using stepwise regression, see Section 5.4) to the combined data from both scaled tanks as well as all of the data from pre-transfer samples as well as the five batch-transfer samples for each of the four base-simulant components. As an illustration, substituting Equation (5.9) into Equation (5.5) yields 


$$
\begin{aligned}
& \ln \left(C_{i j}\right)=b_{0}^{j}+b_{1}^{j}\left(U_{i}\left(\frac{D_{120}}{D_{i}}\right)^{a_{j}}\right)+b_{2}^{j}\left(U_{i}\left(\frac{D_{120}}{D_{i}}\right)^{a_{j}}\right)^{2}+b_{3}^{j}\left(U_{i}\left(\frac{D_{120}}{D_{i}}\right)^{a_{j}}\right)^{3} \\
& +b_{4}^{j} \overline{B S}+b_{5}^{j} \overline{K V}+b_{6}^{j} \overline{K V}^{2}+b_{7}^{j} \overline{C V}+b_{8}^{j}\left[\overline{B S} *\left(U_{i}\left(\frac{D_{120}}{D_{i}}\right)^{a_{j}}\right)\right] \\
& +b_{9}^{j}\left[\overline{B S} *\left(U_{i}\left(\frac{D_{120}}{D_{i}}\right)^{a_{j}}\right)^{2}\right]+b_{10}^{j}[\overline{B S} * \overline{K V}]+b_{11}^{j}\left[\overline{B S} * \overline{K V}^{2}\right] \\
& +b_{12}^{j}\left[\overline{K V} *\left(U_{i}\left(\frac{D_{120}}{D_{i}}\right)^{a_{j}}\right)\right]+b_{13}^{j}\left[\overline{K V}^{2} *\left(U_{i}\left(\frac{D_{120}}{D_{i}}\right)^{a_{j}}\right)\right] \\
& +b_{14}^{j}\left[\overline{K V} *\left(U_{i}\left(\frac{D_{120}}{D_{i}}\right)^{a_{j}}\right)^{2}\right]+b_{15}^{j}\left[\overline{K V}^{2} *\left(U_{i}\left(\frac{D_{120}}{D_{i}}\right)^{a_{j}}\right)^{2}\right] \\
& +b_{16}^{j} B N+b_{17}^{j} B N^{2}+b_{18}^{j}\left[B N *\left(U_{i}\left(\frac{D_{120}}{D_{i}}\right)^{a_{j}}\right)\right]+b_{19}^{j}[B N * \overline{B S}] \\
& +b_{20}^{j}[B N * \overline{K V}]+b_{21}^{j}[B N * \overline{C V}]+b_{22}^{j}\left[B N * \overline{B S} *\left(U_{i}\left(\frac{D_{120}}{D_{i}}\right)^{a_{j}}\right)\right] \\
& +b_{23}^{j}[B N * \overline{B S} * \overline{K V}]+b_{24}^{j}\left[B N * \overline{K V} *\left(U_{i}\left(\frac{D_{120}}{D_{i}}\right)^{a_{j}}\right)\right]+\varepsilon_{i j}
\end{aligned}
$$

where $\quad C_{i j}=$ concentration (lb/gal) of the $j^{\text {th }}$ base-simulant component in a tank with diameter $D_{i}$

$U_{i}=$ mixer-jet nozzle velocity $(\mathrm{ft} / \mathrm{sec})$ in a tank with diameter $D_{i}$

$D_{i}=$ diameter of the $i^{\text {th }} \operatorname{tank}($ in.), where $i=43$ or 120

$\overline{B S}=$ base simulant, coded so that Typical $=-1$ and High $=+1$

$\overline{K V}=$ kinematic viscosity, coded according to Equation (5.3) so that the smallest and largest $K V$ values (over both size tanks) are coded to -1 and +1 , respectively

$\overline{C V}=$ capture velocity, coded according to Equation (5.4) so that the smallest and largest $C V$ values (over both size tanks) are coded to -1 and +1 , respectively

$B N=$ batch number $(k=0,1, \ldots, 5)$ where $k=0$ denotes pre-transfer, and $k=1,2, \ldots, 5$ denote transfer batches $1,2, \ldots, 5$ 


$$
\begin{aligned}
\varepsilon_{i j}= & \text { random error in the concentration of the } j^{\text {th }} \text { base-simulant component a tank with } \\
& \begin{array}{l}
\text { diameter } D_{i} . \\
\text { identically distributed according to the normal (Gaussian) distribution with mean } \\
\\
0 \text { and variance } \sigma^{2}
\end{array} \\
b_{0}^{j} \text { to } b_{24}^{j}= & \text { model coefficients for the } j^{\text {th }} \text { base-simulant component } \\
a_{j}= & \text { scale exponent (coefficient) for the } j^{\text {th }} \text { base-simulant component. }
\end{aligned}
$$

Note that the model in Equation (5.10) has the same form for each base-simulant component, but the model coefficients ( $a_{j}$ and $b_{0}^{j}$ to $b_{24}^{j}$ ) will be different for each base-simulant component $(j)$. There are two main assumptions made in the model of Equation (5.10).

1. The first assumption is that the coefficients $a_{j}$ and $b_{0}^{j}$ to $b_{24}^{j}$ are the same for the two scaled tanks (which is why these coefficients do not have " $i$ " included in the subscript and superscripts). This assumption can be assessed by separately fitting the model in Equation 5.4 to the data for each tank. The results of such data modeling are reported in Section 7, and show that the scaling exponents $a_{i j}$ and coefficients $b_{0}^{i j}$ to $b_{24}^{i j}$ are not always the same for the two scaled tanks.

2. The second assumption is that the coefficient $a_{j}$ for the $j^{\text {th }}$ component is the same for all combinations of test parameters. However, plots of subsets of the data in Section 3.3.2 indicate that this may not be the case. This indication is confirmed in Section 8, where the $a_{j}$ values are calculated as functions of other test parameters.

There are insufficient data in the test matrices (Table 2.3 and Table 2.4) to enable expanding the form of the model in Equation (5.10) to allow for the coefficients to be written as functions of the tank size and other test parameters. Hence, Equation (5.10) is not used in this report as the basis for developing scaling relationships.

Although moot for the above reason, fitting a model like Equation (5.10) to the data is very complicated. As discussed at the end of Section 2.2, the 22 test combinations in Table 2.3 were performed in random order, but the tests in the 43 -inch and 120 -inch tank were performed in the same time period. This is a restriction on randomization on the order of performing the 44 tests, and causes the data to have a split-plot structure. Such a data structure involves two sources of testing and measurement uncertainty, as well as covariance between the results of the 43-inch tank and the 120-inch tank. Yet another complication is that the scaling equation in the model of Equation (5.10) causes the model to be nonlinear in the scale exponent, such that iterative, nonlinear least squares fitting methods are required rather than one-step OLS fitting methods. Combined with the split-plot data structure, the fitting of the model in Equation (5.10) would be very complicated. For more information about these issues, see Section 7.5 of Lee et al. (2012a). 


\subsection{Model Fitting, Model Evaluation, and Sensitivity Assessment}

Section 5.4.1 discusses the stepwise regression approach used to fit the models in Equation (5.5) and Equation (5.8) to the test data for each of the scaled tanks. Section 5.4.2 discusses numeric and graphical methods for evaluating how well a model fits the data. Section 5.4.3 presents the graphical method used to assess the sensitivity of the component concentrations to model parameters.

\subsubsection{Stepwise Regression}

Because the models in Equations (5.5) and (5.8) likely contain more terms than are needed, stepwise regression using OLS was used to develop model forms consisting of subsets of terms in Equations (5.5) and (5.8). Stepwise regression first selects the model term (among those possible in Equation (5.5) or Equation (5.8) that most reduces the sum of squared errors (SSE) ${ }^{(a)}$ compared to a constant (i.e., intercept only) model. Then, in subsequent iterations, the remaining model term that most reduces the SSE is selected. During each iteration of the stepwise regression process in which the model contains two or more terms, all of the terms in the model are checked for statistical significance. Any terms not statistically significant are removed from the model before starting the next iteration to add another model term. Because there are correlations among model terms, it is possible for terms to initially enter the model as statistically significant, but then later leave the model when no longer statistically significant because of other terms that have since entered the model. The iterative process of stepwise regression stops when there are no longer any terms that meet the statistical significance criterion set for adding new terms to the model, and when no terms in the model fail the statistical significance criterion for a term to remain in the model. The statistical significance criterion for model terms to enter or leave the model was varied from 0.10 to 0.15 (which are probabilities of incorrectly including a term in a model). However, ultimately only terms with statistical significance $<0.05$ are typically retained in a model.

Stepwise regression methods can sometimes produce models that are not hierarchical, which means there are higher-order terms without the corresponding lower-order terms. Examples of a nonhierarchical model are when it contains: (i) $\overline{K V}^{2}$ without containing $\overline{K V}$, (ii) $\overline{B S} * \overline{K V}$ without containing $\overline{B S}$ or $\overline{K V}$, or (iii) $B N * \overline{B S} * \overline{K V}$ without containing at least one of the three possible twoparameter interactions. Non-hierarchical models can cause certain problems, especially when the model is developed using coded parameters. Hence, terms necessary to obtain a hierarchical model are manually added to the model. Such terms may not be statistically significant, but this is not a problem given that hierarchical models avoid other kinds of problems.

The results of a stepwise regression presented in this report are the terms in the model, the coefficients of the model terms, the standard deviations of the coefficients, and the p-value of the coefficient. The p-value is the probability (and hence a number between 0 and 1.0) of incorrectly deciding that the model coefficient is statistically different from zero. Typically, p-values less than 0.05 are desirable, although sometimes coefficients with p-values as large as 0.10 may be retained in a model.

(a) Here error is prediction error, namely the predicted value of the response variable minus the measured value of the response variable. In Equations (5.5) and (5.8), the response variable is $\ln (C j)$. 


\subsubsection{Evaluating How Well a Model Fits the Data}

This section presents several numerical summaries and one graphical method to assess how well a model fits the corresponding data. The graphical summary is referred to as a predicted versus measured $(\mathrm{PvM})$ plot, because it plots the model predicted values $\left[\ln \left(\hat{C}_{j}\right)\right]$ (on the $y$-axis) versus the data values $\left[\ln \left(C_{j}\right)\right]$ (on the $x$-axis) for the data points used to fit a model. In addition to the data points used to fit a model, a PvM plot includes the line $y=x$, which corresponds to the model perfectly predicting the data values. Of interest in PvM plots is (i) whether there is a tendency for the model to overpredict or underpredict for certain ranges of concentration values and (ii) the extent of the random scatter of the data points around the $y=x$ line. The former provides information about concentrations for which the model may yield biased predictions, while the latter provides information about the random uncertainty in model predictions.

The following numerical summaries are defined specifically for the models in Equation (5.5) and Equation (5.8), which predict $\ln \left(C_{j}\right)$.

The $\mathrm{R}^{2}$ statistic is calculated by

$$
\mathrm{R}^{2}=1-\frac{\sum_{h=1}^{n}\left[\left(\ln \left(\hat{C}_{j h}\right)-\ln \left(C_{j h}\right)\right)\right]^{2}}{\sum_{h=1}^{n}\left[\ln \left(C_{j h}\right)-\overline{\ln \left(C_{j .}\right)}\right]^{2}}
$$

where $\ln \left(\hat{C}_{j h}\right)=$ model prediction of the natural logarithm of concentration $(\mathrm{lb} / \mathrm{gal})$ of the $j^{\text {th }}$ basesimulant component for the $h^{\text {th }}$ data point used to fit a model $\ln \left(C_{j h}\right)=$ natural logarithm of the measured concentration (lb/gal) of the $j^{\text {th }}$ base-simulant component for the $h^{\text {th }}$ data point used to fit a model

$\overline{\ln \left(C_{j .}\right)}=$ mean of the natural logarithms of measured concentrations (lb/gal) of the $j^{\text {th }}$ basesimulant component over the $h=1,2, \ldots, n$ data points used to fit a model $n=$ number of data points used to fit a model.

$\mathrm{R}^{2}$ is interpreted as the fraction of variation in the $\ln \left(C_{j h}\right)$ values of a data set that is accounted for by the fitted model.

The adjusted $R^{2}$ statistic $\left(R_{A}^{2}\right)$ is calculated by

$$
\mathrm{R}_{\mathrm{A}}^{2}=1-\frac{\sum_{h=1}^{n}\left[\left(\ln \left(\hat{C}_{j h}\right)-\ln \left(C_{j h}\right)\right)\right]^{2} /(n-p)}{\sum_{h=1}^{n}\left[\ln \left(C_{j h}\right)-\overline{\ln \left(C_{j .}\right)}\right]^{2} /(n-1)}
$$

where $p$ is the number of coefficients estimated in fitting the model, and all other notation is as defined following Equation (5.11). $\mathrm{R}_{\mathrm{A}}^{2}$ can be thought of as a version of $\mathrm{R}^{2}$ that includes a penalty for the 
number of model coefficients fitted to the data ( $p$ ). If too many unnecessary terms (and coefficients) are included in a model, then $\mathrm{R}_{\mathrm{A}}^{2}$ will be noticeably smaller than $\mathrm{R}^{2}$.

The root mean squared error (RMSE) is calculated by

$$
\operatorname{RMSE}=\left(\frac{\sum_{h=1}^{n}\left[\ln \left(\hat{C}_{j h}\right)-\ln \left(C_{j h}\right)\right]^{2}}{n-p}\right)^{0.5}
$$

where all notation is as previously defined. When the fitted model does not have a statistically significant lack of fit (LOF), the RMSE estimates the experimental and measurement standard deviation associated with performing the tests and measuring the response variable (i.e., $\left.\ln \left(C_{j h}\right)\right)$.

Finally, because the data sets used to fit the models contain replicates ${ }^{(a)}$, the model LOF can be statistically assessed by considering the p-value corresponding to the $F$-statistic:

$$
F_{L O F}=\frac{S S_{L O F} / d f_{L O F}}{S S_{P E} / d f_{P E}}=\frac{\sum_{h=1}^{n}\left[\ln \left(\hat{C}_{j h}\right)-\ln \left(C_{j h}\right)\right]^{2} /\left(n-p-d f_{P E}\right)}{\left.\sum_{u=1}^{U} \sum_{v=1}^{V}\left[\ln \left(C_{j u v}\right)-\overline{\ln \left(C_{j u .}\right.}\right)\right]^{2} / N_{S}\left(N_{R}-1\right)}
$$

where $\quad S S_{L O F}=$ sum of squares due to model LOF

$$
\begin{aligned}
d f_{L O F} & =\text { degrees of freedom associated with model LOF } \\
S S_{P E} & =\text { pure error sum of squares } \\
d f_{P E} & =\text { pure error degrees of freedom } \\
N_{S} & =\text { number of replicate sets of data for a given scale tank }\left(N_{S}=4\right) \\
N_{R} & =\text { number of replicates in a replicate set }\left(N_{R}=2\right)
\end{aligned}
$$

and all other notation is as previously defined.

The p-value for the model LOF assessment is the probability (between 0 and 1.0) of being incorrect if the model LOF is declared to be statistically significant. Values less than 0.05 indicate a small probability of being wrong if a model is found to have a statistically significant LOF. The larger p-values get, the more confidence that there is no model LOF. For more information about the statistical methodology for assessing model LOF, see Myers and Montgomery (1995, Section 2.7.4).

(a) As discussed in Section 2.2, the experimental design in Table 2.3 contained four replicate pairs for tests done at each of the two test scales. However, because actual values of the test parameters were used in fitting the models rather than the target values, the replicate pairs are not exactly replicates. This could lead to slightly over-estimating the Pure Error Sum of Squares in the denominator of Equation (5.14), which may slightly decrease the likelihood of declaring a model LOF statistically significant. However, it is expected that such effects are minimal, and the model LOF results are relied upon as the best indication whether the fitted models have a statistically significant LOF. 


\subsubsection{Assessing the Sensitivity of Component Concentrations to Variations in the Test Parameters}

It is of interest to assess how sensitive the component concentration $C_{j}\left(j=\right.$ gibbsite, $\mathrm{SS}$, sand, $\left.\mathrm{ZrO}_{2}\right)$ is to variations in the test parameters. The sensitivity of $C_{j}$ to a specific test parameter is not easily determined from a fitted model because there may be multiple terms involving a specific test parameter in a model. Also, the fact that some test parameters are coded and others are not makes it difficult to assess sensitivity by considering only model coefficients.

A graphical method referred to as a prediction profile plot (PPP) was used in this report to display the sensitivity of a component concentration $C_{j}$ to the parameters $(U, B S, K V$, and $C V$ ) used in the models of Equation (5.5) and Equation (5.8) for the two scaled tanks. The PPPs used in this report display model predictions of $C_{j}$ as functions of varying all four model parameters. The three model parameters $U, K V$, and $C V$ were varied in each plot and the $B S$ was held constant (different plots for the different $B S$ types). The models in Equation (5.5) and Equation (5.8) predict $\ln \left(C_{j}\right)$, but those predictions can be converted to $C_{j}$ via

$$
C_{j}=e^{\ln \left(C_{j}\right)}
$$

Prediction profiles are obtained by applying a fitted model to predict $C_{j}$ values for a range of values of one model parameter (say Parameter A) while holding constant all other model parameters. Profiles are thus obtained for a few (2-5) values of a second model parameter (say Parameter B) while holding constant all parameters other than Parameters A and B. Then, the profiles showing $C_{j}$ as a function of Parameter A are plotted for each of the 2-5 values of Parameter B. ${ }^{(a)}$ Predicted values of $C_{j}$ are shown on the $y$-axis, values of Parameter A are shown on the $x$-axis, and the 3-5 values of Parameter B are represented by the multiple prediction profiles (typically plotted using different line types or colors, with a legend to identify the values or settings of Parameter B.

In a PPP, the more sensitive $C_{j}$ is to Parameter $\mathrm{A}$ (the one displayed on the $x$-axis), the steeper the prediction profiles. Nearly horizontal profiles indicate there is little effect of Parameter $\mathrm{A}$ on $C_{j}$. The more sensitive $C_{j}$ is to Parameter B (values of which are represented by different line types or colors in the plots, the more distance there is between the lines. PPPs are also useful in assessing the degree to which Parameters $\mathrm{A}$ and $\mathrm{B}$ have an interactive effect on $C_{j}$. Interaction between Parameters $\mathrm{A}$ and $\mathrm{B}$ will show up as non-parallelism of the profiles in the PPP.

Some features of a PPP can be anticipated based on the terms in the fitted model used to produce the PPP. For example, if a parameter does not appear in any term of a model, then clearly that parameter is predicted to have no effect on $C_{j}$, and the prediction profiles will reflect that. If it is Parameter A that does not appear in the model, then the prediction profiles will be horizontal lines (assuming Parameter $\mathrm{B}$ does appear in the model). If Parameter A appears in the model but not Parameter B, then the prediction profiles will lie on top of one another (because Parameter B is predicted to have no effect on $C_{j}$ ). If a model does not contain any terms involving Parameter A and Parameter B together, then those two

\footnotetext{
(a) This description of the method for calculating prediction profiles presumes that the model parameters can be varied independently of one another. If this is not the case, such as when two parameters have some degree of linear or curvilinear correlation, then more complicated methods that account for the correlation are required to obtain prediction profiles.
} 
parameters have no interactive effect on $C_{j}$ and the prediction profiles for those two parameters will be parallel. 


\subsection{Equivalent Performance Approach to Calculating Scaling Exponents}

This section presents the EP approach used in this report to calculate scaling exponents for various combinations of test parameter values. Section 6.1 presents a simple scaling equation with a scaling exponent, while Section 6.2 discusses the EP approach to calculating scale exponents.

\subsection{Scaling Equation and Exponent}

Assume that a MTP metric, in this report the concentration of the $j^{\text {th }}$ base-simulant component, can be expressed via the power-law model

$$
C_{i j}=a_{j 0} U_{i}^{a_{j 1}} D_{i}^{a_{j 2}}
$$

where $\quad C_{i j}=$ concentration of the $j^{\text {th }}$ component of the base simulant in the $i^{\text {th }}$ tank (lb/gal)

$$
\begin{aligned}
U_{i} & =\text { mixer-jet nozzle velocity }(\mathrm{ft} / \mathrm{sec}) \text { for the } i^{\text {th }} \operatorname{tank} \text {, with " } i \text { " }=43 \text { or } 120 \\
D_{i} & =\text { diameter of the } i^{\text {th }} \operatorname{tank}(\text { inches), with “ } i \text { " }=43 \text { or } 120 \\
a_{j 0}, a_{j 1}, a_{j 2} & =\text { coefficients of the power-law model }
\end{aligned}
$$

Then substituting $U_{43}$ and $D_{43}$ into Equation (6.1) yields an equation for $C_{43, j}$, while substituting $U_{120}$ and $D_{120}$ into Equation (6.1) yields an equation for $C_{120, j}$. For EP (i.e., equal component concentrations) at the two scales, the equations for $C_{43, j}$ and $C_{120, j}$ must be equal. The combined equation can be re-expressed to yield a simple, geometric-scaling equation for tank mixing and transfer:

$$
U_{120}=U_{43}\left(\frac{D_{120}}{D_{43}}\right)^{a}
$$

where $D_{43}$ and $D_{120}$ denote the diameter of the 43-inch and 120-inch tanks, respectively; $U_{43}$ denotes a specific mixer-jet nozzle velocity for the 43-inch tank; and $U_{120}$ denotes the mixer-jet nozzle velocity for the 120-inch tank that gives EP, given the scaling exponent $(a)$.

If the scaling exponent in Equation (6.2) is not known, then it is conceptually possible to estimate it by experimentally determining the velocity in each of the two tanks that produces an equivalent MTP. For example, using the 43-inch and 120-inch tanks in the SSMD, the velocity in one tank could be experimentally identified that produces MTP equivalent to the MTP at a specific velocity in the other tank. The scaling exponent could then be calculated based on the tank diameters and the identified velocities. Ideally, many different velocities would be compared at the two scales to provide a better estimate of the scale exponent. However, such an approach would require a large amount of testing, more than would be practical.

Alternatively, models of MTP as a function of mixer-jet nozzle velocity (with all other parameters held constant) for the two scaled tanks could be used to calculate pairs of velocities that provide 
equivalent MTP in the two tanks. Using MTP models for each of the two scaled tanks allows for obtaining a much greater number of velocity pairs that yield equivalent MTP. Each velocity pair could be used with Equation (6.2) to estimate the scaling exponent $(a)$. This would provide for assessing whether " $a$ " is constant or varies over a range of velocity pairs. Section 6.2 describes using models for each scaled tank (of the forms presented in Section 5.2 and 5.3) to apply the EP approach.

\subsection{Equivalent Performance Approach to Calculating Scaling Exponents}

This section describes using reduced forms of the models for $\ln \left(C_{j}\right)$ in Equation (5.5) or Equation (5.8) to implement the EP approach for calculating values of the scaling exponent $(a)$. Those models express $\ln \left(C_{j}\right)$ as functions of $U, \overline{B S}, \overline{K V}$, and $\overline{C V}$ with separate models for each of the two scaled tanks. A pair of separate models for the two scaled tanks, either of the form in Equation (5.5) or the form in Equation (5.8), can be used to perform the following calculations.

1. Specify a grid of points consisting of allowable combinations of $B S, K V, C V$, and $B N$ that meet (i) the minimums and maximums of $K V$ and $C V$ actual test values listed in Table 3.1 and (ii) the constraint of allowable combinations of $(S V, C V)$ in Equation (2.1). The constraint on the combinations of $S V$ and $C V$ in Equation (2.1) is converted to the following constraint on combinations of $K V$ and $C V$ using the definition of $K V=S V /$ Density $^{\left({ }^{(a)} \text { : }\right.}$

$$
4948.16 \sqrt{2.17 C V}\left(\frac{1}{K V}\right)<2200 \text {. }
$$

The number of levels of $K V$ and $C V$ should be enough to assess curvature and interaction effects on scale exponents (as discussed subsequently), but not so large as to provide a burdensome number of calculations and evaluations. Note that the grid is not a rectangular "box" in three dimensions because of the constraint on $(\mathrm{KV}, \mathrm{CV})$ combinations in Equation (6.3).

2. For each grid point, code $B S=$ Typical as -1 and $B S=$ High as +1 . Also, convert the values of $K V$ and $C V$ to coded values of $\overline{K V}$ and $\overline{C V}$ using Equations (5.3) and (5.4), respectively.

3. For each grid combination of $\overline{B S}, \overline{K V}, \overline{C V}$, and $B N$, perform Steps 4 through 6 for each basesimulant component $\left(j=\right.$ gibbsite, $\mathrm{SS}$, sand, and $\mathrm{ZrO}_{2}$ ).

4. Substitute each of a series of $U_{43}$ values [denoted $U_{43, j, 1}, U_{43, j, 2}, \ldots, U_{43, j, n}$ ] in the range tested (see Table 3.1) into the version of either Equation (5.5) or Equation (5.8) fitted to the data for the 43-inch tank (for a given " $j$ ") to calculate the corresponding series of $C_{43, j}$ values [denoted $\left.C_{43, j, 1}, C_{43, j, 2}, \ldots, C_{43, j, n}\right]$ for the specific combination of $\overline{B S}, \overline{K V}, \overline{C V}$, and $B N$ values.

5. Take the version of Equation (5.5) or Equation (5.8) fitted to the data for the 120-inch tank for the $j^{\text {th }}$ component and set the left side $\left(C_{120, j}\right)$ equal, in turn, to each of the values in the $C_{43, j, 1}, C_{43, j, 2}$,

\footnotetext{
(a) Note that this substitution bypasses the incorrect linear relationship between supernatant viscosity and density
} discussed in Section 2.1. 
$\ldots, C_{43, j, n}$ series obtained in Step 4. Then, for each specific combination of $\overline{B S}, \overline{K V}, \overline{C V}$, and $B N$ for the $j^{\text {th }}$ component, solve each resulting equation for the value of $U_{120, j}$ that gives EP (namely $C_{120, j}=C_{43, j}$ ). The resulting series of values is denoted $U_{120, j, 2}, \ldots, U_{120, j, n}$. This series of $U_{120, j}$ values should be compared to the range of values investigated during testing with the 120-inch tank (see Table 3.1) because any values falling outside that range will be extrapolative model predictions. The result is a series of $\left(U_{43, j}, U_{120, j}\right)$ pairs for the specific $j^{\text {th }}$ component and a given combination of uncoded $B S, K V, C V$, and $B N$ values that yield EP in the two tank sizes. This series of equivalent-performance velocity pairs is denoted $\left(U_{43, j, 1}, U_{120, j, 1}\right),\left(U_{43, j, 2}, U_{120, j, 2}\right), \ldots$ , $\left(U_{43, j, n}, U_{120, j, n}\right)$ for the $j^{\text {th }}$ component and a given combination of $B S, K V, C V$, and $B N$ values.

6. For each of the velocity pairs for a given $j^{\text {th }}$ component and a given combination of $B S, K V, C V$, and $B N$ in Step 5, use Equation (6.1) and solve for the corresponding scaling exponent $(a)$ value. The general solution of Equation (6.1) for the exponent, using the notation here, is

$$
a_{j, h}=\frac{\ln \left(U_{120, j, h)} / U_{43, j, h}\right)}{\ln \left(D_{120} / D_{43}\right)}
$$

where "In" denotes the natural logarithm and $h=1,2, \ldots, n$. The resulting solutions are the scaling exponents for the $j^{\text {th }}$ component corresponding to specific combinations of $B S, K V, C V$, and $B N$ for the series of velocity pairs in Step 5 that yield equivalent performance, as calculated by separate fitted models for each of the two scaled tanks [Equation (5.5) or Equation (5.8)].

7. At this step, for every grid combination of $B S, S V, C V$, and $B N$ in Step 1, there is a series of estimated scaling exponents $\left(a_{j, 1}, a_{j, 2}, \ldots, a_{j, n}\right)$ corresponding to the series of equivalentperformance velocity pairs $\left[\left(U_{43, j, 1}, U_{120, j, 1}\right),\left(U_{43, j, 2}, U_{120, j, 2}\right), \ldots,\left(U_{43, j, n}, U_{120, j, n}\right)\right]$ for the $j^{\text {th }}$ component.

In Step 5, any pair $\left(U_{43, j, h}, U_{120, j, h}\right)$ that includes an $U_{120, j, h}$ value outside of its test data range is questionable because it involves a form of extrapolation outside the data set. Such pairs often resulted in very odd (larger negative or positive " $a$ " values). Hence, it was decided to eliminate such pairs $\left(U_{43, j, h}, U_{120, j, h}\right)$ and corresponding " $a$ " values from the data analyses (in Section 8).

The $a_{j, h}(h=1,2, \ldots, n)$ values for the $j^{\text {th }}$ component and the series of EP velocity pairs (for each grid combination of $B S, S V, C V$, and $B N$ ) can then be treated as "response variables" and evaluated as to how sensitive they are to $U_{43}$ (or equivalently $U_{120}$ ), $B S, K V, C V$, and $B N$. These evaluations are performed in Section 8.1 (for the models in Section 7.1) and Section 8.2 (for the models in Section 7.2).

Ideally the uncertainties associated with calculated values of $a_{j, m}(m=1,2, \ldots, n)$ for each grid combination of $B S, S V, C V$, and $B N$ would be determined and reported with the corresponding $a_{j, m}$ value. However, the preceding calculation steps are complicated and involve separate models for concentrations of the $j^{\text {th }}$ component in the 43-inch and 120-inch tanks. Also, the split-plot structure of the data discussed at the end of Section 5.3 may have to be accounted for in calculating uncertainties of $a_{j, m}$ values. Hence, it was not possible to evaluate the uncertainties of the $a_{j, m}$ as part of the work documented in this report. 


\subsection{Results of Modeling Base-Simulant Component Concentrations as Functions of Several Parameters}

Section 7.1 presents the results from fitting reductions of the model form in Equation (5.5), while Section 7.2 presents the results from fitting reductions of the model form in Equation (5.8). Recall that those model forms are functions of $U, \overline{B S}, \overline{K V}, \overline{C V}$, and $B N$, where the "bar" notation indicates coded values of the parameters are used, as discussed in Section 5.1.1. Because the models are a function of $B N$, the data from pre-transfer samples and all five transfer-batch samples are combined and jointly used to fit the reductions of the models in Equations (5.5) and (5.8). Models were fit separately for the data sets corresponding to the 43 -inch and 120 -inch tanks, for each of the four component concentrations (gibbsite, $\mathrm{SS}$, sand, and $\mathrm{ZrO}_{2}$ ). The reduced forms of the models in Equations (5.5) and (5.8) were obtained using stepwise regression, as described in Section 5.4.1. The statistics and plots that summarize how well models fit the data, as discussed in Section 5.4.2, are presented for each model.

\subsection{Results of Fitting Models for Component Concentrations as Functions of the Test Parameters with Up to a Cubic Polynomial Dependence on Jet Velocity}

Sections 7.1.1 to 7.1.4 present the results of applying stepwise regression to obtain reductions of the model in Equation (5.5) for concentrations of gibbsite, SS, sand, and $\mathrm{ZrO}_{2}$, respectively. For each component, separate models were fit to data from the 43 -inch tank and the 120 -inch tank.

\subsubsection{Models for Gibbsite Concentrations as Functions of the Test Parameters with Up to a Cubic Polynomial Dependence on Jet Velocity}

Table 7.1 presents the model terms, estimated coefficients, coefficient standard deviations, and coefficient p-values for the models of the form in Equation (5.5) obtained by stepwise regression when separately fitting gibbsite concentration data for the 43-inch and 120-inch tanks. Also included in Table 7.1 are the values of RMSE, $\mathrm{R}^{2}$, and $\mathrm{R}_{\mathrm{A}}^{2}$ for each model, as well as the LOF p-value (see Section 5.4.2).

Table 7.1 shows that the $\ln \left(C_{\text {gibbsite }}\right)$ model for the 43 -inch tank has only $B S$ as a predictor variable. Still, the model accounts for a high fraction of the variation in the data $\left(\mathrm{R}^{2}=0.9940\right)$ and does not have a statistically significant LOF (p-value $=0.7102)$. The $\ln \left(C_{\text {gibbsite }}\right)$ model for the 120 -inch tank has linear effects of $\overline{B S}, \overline{K V}$, and $\overline{C V}$, as well as a quadratic effect of $\overline{K V}$. This model has $\mathrm{R}^{2}=0.9940$ and does not have a statistically significant LOF (p-value $=0.3430$ ). Finally, note that neither model in Table 7.1 depends on $U$. Hence, it is not possible to use the EP approach described in Section 6.2 to calculate scaling exponents. 
Table 7.1. Model Terms, Estimated Coefficients, Coefficient Standard Deviations, Coefficient p-Values, and Model Summary Statistics for the Gibbsite Concentration Models, Corresponding to the 43-inch and 120-inch Tanks, of the Form in Equation (5.5) Obtained by Stepwise Regression

\begin{tabular}{|c|c|c|c|c|c|}
\hline $\begin{array}{c}\ln \left(\mathbf{C}_{\text {gibbsite }}\right) \\
\text { Model Term }^{(a)}\end{array}$ & $\begin{array}{l}\text { Coefficient } \\
\text { Estimate }\end{array}$ & SD(Coefficient) & p-value ${ }^{(b)}$ & Significance $^{(c)}$ & $\begin{array}{c}\text { Model Fit Summary } \\
\text { Statistics }\end{array}$ \\
\hline \multicolumn{6}{|c|}{ 43-inch Tank } \\
\hline Intercept & -1.5469 & 0.0100 & $<0.0001$ & $* * * *$ & $\mathrm{RMSE}=0.1248$ \\
\hline \multirow[t]{3}{*}{$\overline{B S}$} & -1.6075 & 0.0100 & $<0.0001$ & $* * * *$ & $\mathrm{R}^{2}=0.9940$ \\
\hline & & & & & $\mathrm{R}_{\mathrm{A}}^{2}=0.9940$ \\
\hline & & & & & LOF $p$-value $=0.7102$ \\
\hline \multicolumn{6}{|c|}{ 120-inch Tank } \\
\hline Intercept & -1.5973 & 0.0187 & $<0.0001$ & $* * * *$ & $\mathrm{RMSE}=0.1491$ \\
\hline$\overline{B S}$ & -1.5920 & 0.0128 & $<0.0001$ & $* * * *$ & $\mathrm{R}^{2}=0.9916$ \\
\hline$\overline{K V}$ & -0.0752 & 0.0283 & 0.0087 & $* *$ & $\mathrm{R}_{\mathrm{A}}^{2}=0.9914$ \\
\hline$\overline{C V}$ & 0.1056 & 0.0197 & $<0.0001$ & $*$ & LOF $p$-value $=0.3430$ \\
\hline$\overline{K V}^{2}$ & 0.0931 & 0.0328 & 0.0051 & $* *$ & \\
\hline
\end{tabular}

(a) The model terms were selected by stepwise regression or were added to make the model hierarchical (see Section 5.4.1).

(b) The probability of incorrectly concluding that the coefficient estimate is different from zero. Low values (e.g., $<0.05$ and especially $<0.01)$ indicate coefficient estimates that are statistically different from zero.

(c) $*=$ p-value $<0.05, * *=$ p-value $<0.01, * * *=p$-value $<0.001$, and $* * * *$ p-value $<0.0001$. Also, note that the main (individual) effect of a given parameter may not be statistically significant if that parameter is involved in one or more statistically significant interactions.

Figure 7.1 displays the PvM plots (see Section 5.4.2) corresponding to the gibbsite concentration models in Table 7.1 for the 43-inch and 120-inch tanks. Both panels in Figure 7.1 show two clusters of points, which is a result of the very different gibbsite concentrations in the two base simulants (see Table 2.2). Even though the model for the 120 -inch tank contains statistically significant terms involving $\overline{K V}$ and $\overline{C V}$, the bottom panel of Figure 7.1 shows that these parameters have very small effects compared to the effect of $\overline{B S}$. 

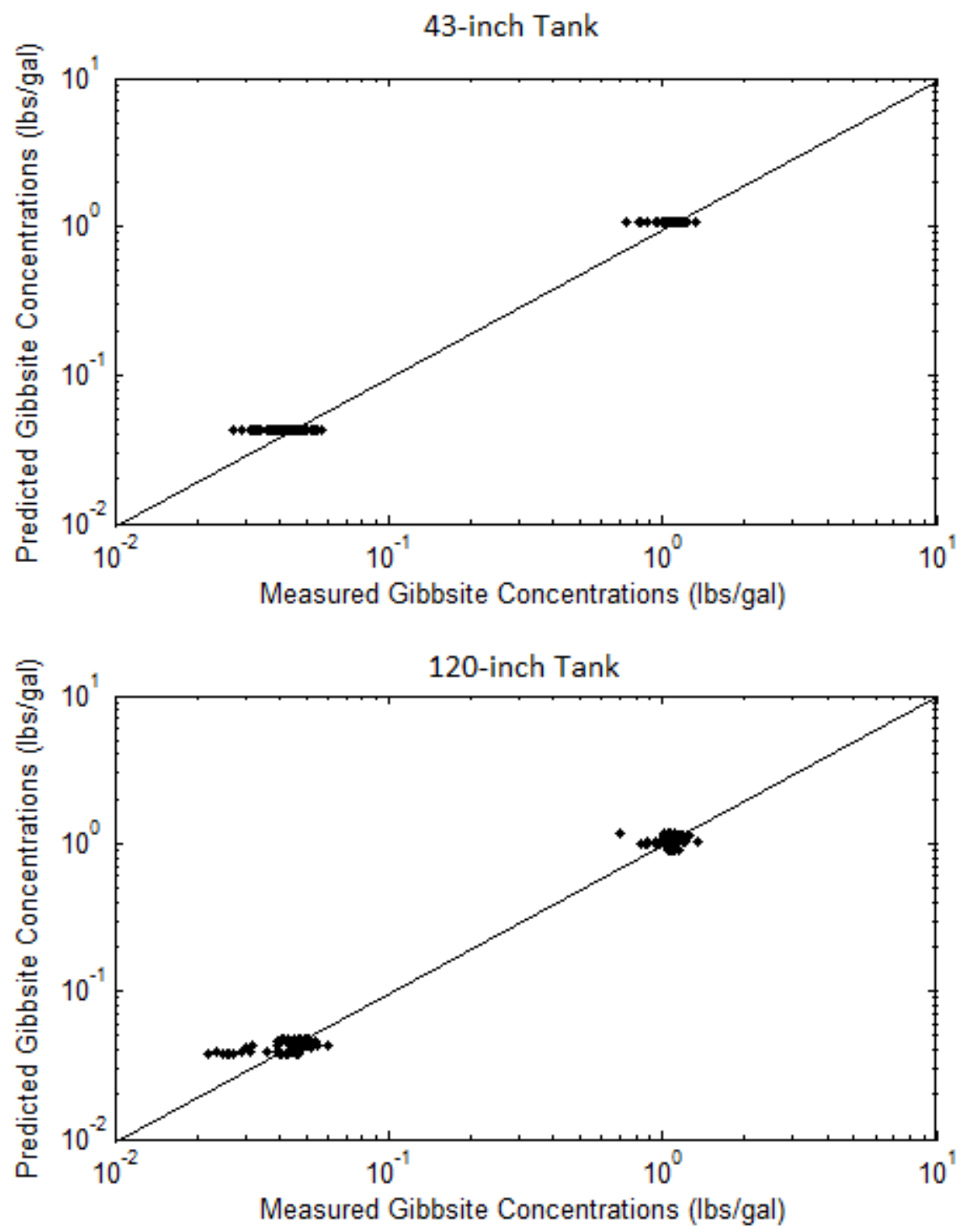

Figure 7.1. Predicted versus Measured Plots for the Gibbsite Concentration Models from Table 7.1 for the 43-inch Tank and 120-inch Tank (For Information Only)

\subsubsection{Models for Stainless Steel Concentrations as Functions of the Test Parameters with Up to a Cubic Polynomial Dependence on Jet Velocity}

Table 7.2 presents the model terms, estimated coefficients, coefficient standard deviations, and coefficient p-values for the models of the form in Equation (5.5) obtained by stepwise regression when separately fitting SS concentration data for the 43-inch and 120-inch tanks. Also included in Table 7.2 are the values of RMSE, $\mathrm{R}^{2}$, and $\mathrm{R}_{\mathrm{A}}^{2}$ for each model, as well as the LOF $\mathrm{p}$-value (see Section 5.4.2). The model for the 43-inch tank was fitted after eliminating two influential outliers, corresponding to Batch 1 for Test Combination 22 and Batch 5 of Test Combination 18 (see Figure B.6). The model for the 120- 
inch tank was obtained after eliminating the pre-transfer observation for Test Combination 26 as an outlier (see Figure B.2 and Figure B.6).

Table 7.2 shows that the $\ln \left(C_{S S}\right)$ models for the 43 -inch and 120 -inch tanks involve a larger number of significant terms in the models, with both models depending on $U$. Both models account for high fractions of the observed variability of $\ln \left(C_{S S}\right)$ values (the $\mathrm{R}^{2}$ values are above 0.93 ) and do not have statistically significant LOFs (p-values $=0.6847$ and 0.1848 ).

Figure 7.2 displays the PvM plots (see Section 5.4.2) corresponding to the SS concentration models in Table 7.2 for the 43-inch and 120-inch tanks. The PvM plot for the 43-inch model shows that the model may slightly overpredict SS concentrations below $\sim 0.02 \mathrm{lb} /$ gal but otherwise predicts accurately (within the testing uncertainty). The PvM plot for the 120-inch tank has a tendency to overpredict SS concentrations (i) below $0.02 \mathrm{lb} / \mathrm{gal}$ (although there were only three data points with a concentration that low) and (ii) in the range $0.09-0.25 \mathrm{lb} / \mathrm{gal}$.

Table 7.2. Model Terms, Estimated Coefficients, Coefficient Standard Deviations, Coefficient p-Values, and Model Summary Statistics for the Stainless Steel Concentration Models, Corresponding to the 43-inch and 120-inch Tanks, of the Form in Equation (5.5) Obtained by Stepwise Regression

\begin{tabular}{|c|c|c|c|c|c|}
\hline $\begin{array}{c}\ln \left(C_{S S}\right) \\
\text { Model Term }^{(a)}\end{array}$ & $\begin{array}{l}\text { Coefficient } \\
\text { Estimate }\end{array}$ & SD(Coefficient) & p-value ${ }^{(b)}$ & Significance ${ }^{(\mathrm{c})}$ & $\begin{array}{c}\text { Model Fit Summary } \\
\text { Statistics }\end{array}$ \\
\hline \multicolumn{6}{|c|}{ 43-inch Tank } \\
\hline Intercept & -14.4292 & 1.0270 & $<0.0001$ & $* * * *$ & RMSE $=0.3809$ \\
\hline$U$ & 0.6522 & 0.0789 & $<0.0001$ & $* * * *$ & $\mathrm{R}^{2}=0.9318$ \\
\hline$\overline{B S}$ & 1.0762 & 0.0412 & $<0.0001$ & $* * * *$ & $\mathrm{R}_{\mathrm{A}}^{2}=0.9265$ \\
\hline$\overline{K V}$ & -0.5828 & 0.0906 & $<0.0001$ & $* * * *$ & LOF $p$-value $=0.6847$ \\
\hline$\overline{C V}$ & 0.1625 & 0.0543 & 0.0033 & $* *$ & \\
\hline$B N$ & -0.2750 & 0.0953 & 0.0045 & $* *$ & \\
\hline$U^{2}$ & -0.0085 & 0.0015 & $<0.0001$ & $* * * *$ & \\
\hline$\overline{K V}^{2}$ & -12.8886 & 2.1615 & $<0.0001$ & $* * * *$ & \\
\hline$\overline{B S} * \overline{K V}$ & 0.2993 & 0.0652 & $<0.0001$ & $* * * *$ & \\
\hline$U * \overline{K V}^{2}$ & 0.9986 & 0.1610 & $<0.0001$ & $* * * *$ & \\
\hline$U^{2} * \overline{K V}^{2}$ & -0.0189 & 0.0029 & $<0.0001$ & $* * * *$ & \\
\hline$U * B N$ & 0.0070 & 0.0035 & 0.0486 & $*$ & \\
\hline $\begin{array}{l}\text { (a) The model term } \\
\text { Section 5.4.1) } \\
\text { (b) The probability } \\
<0.05 \text { and espe } \\
\text { (c) } *=\text { p-value }<0 \text {. } \\
\text { the main (indivi } \\
\text { involved in one }\end{array}$ & $\begin{array}{l}\text { re selected by } \\
\text { correctly con } \\
y<0.01) \text { indic } \\
*=\text { p-value }< \\
\text { effect of a gi } \\
\text { ore statistical }\end{array}$ & $\begin{array}{l}\text { pwise regression } \\
\text { ding that the coeff } \\
\text { coefficient estims } \\
01, * * *=p \text {-value } \\
\text { parameter may } n \\
\text { ignificant interact }\end{array}$ & $\begin{array}{l}\text { were added } \\
\text { ient estimat } \\
\text { es that are st } \\
0.001 \text {, and * } \\
t \text { be statistice } \\
\text { ns. }\end{array}$ & $\begin{array}{l}\text { to make the mo } \\
\text { is different fron } \\
\text { tatistically differ } \\
* * * *=\text { p-value }< \\
\text { ally significant if }\end{array}$ & $\begin{array}{l}\text { el hierarchical (see } \\
\text { zero. Low values (e.g., } \\
\text { nt from zero. } \\
0.0001 \text {. Also, note that } \\
\text { that parameter is }\end{array}$ \\
\hline
\end{tabular}


Table 7.2. Model Terms, Estimated Coefficients, Coefficient Standard Deviations, Coefficient p-Values, and Model Summary Statistics for the Stainless Steel Concentration Models, Corresponding to the 43-inch and 120-inch Tanks, of the Form in Equation (5.5) Obtained by Stepwise Regression (cont.)

\begin{tabular}{|c|c|c|c|c|c|}
\hline $\ln _{\text {Model Term }^{(\mathbf{a})}}$ & $\begin{array}{l}\text { Coefficient } \\
\text { Estimate }\end{array}$ & SD(Coefficient) & p-value ${ }^{(b)}$ & Significance $^{(c)}$ & $\begin{array}{c}\text { Model Fit Summary } \\
\text { Statistics }\end{array}$ \\
\hline \multicolumn{6}{|c|}{ 120-inch Tank } \\
\hline Intercept & -105.5940 & 25.0603 & $<0.0001$ & $* * * *$ & RMSE $=0.2483$ \\
\hline$U$ & 9.2914 & 2.2365 & 0.0001 & $* * *$ & $\mathrm{R}^{2}=0.9323$ \\
\hline$\overline{B S}$ & 10.0365 & 2.5796 & 0.0002 & $* * *$ & $\mathrm{R}_{\mathrm{A}}^{2}=0.9239$ \\
\hline$\overline{K V}$ & 3.5110 & 0.6734 & $<0.0001$ & $* * * *$ & LOF $p$-value $=0.1848$ \\
\hline$\overline{\overline{C V}}$ & 0.1695 & 0.0349 & $<0.0001$ & $* * * *$ & \\
\hline$B N$ & -0.1093 & 0.0133 & $<0.0001$ & $* * * *$ & \\
\hline$U^{2}$ & -0.2794 & 0.0663 & $<0.0001$ & $* * * *$ & \\
\hline$U^{3}$ & 0.0028 & 0.0007 & $<0.0001$ & $* * * *$ & \\
\hline$\overline{K V}^{2}$ & -51.5734 & 7.0283 & $<0.0001$ & $* * * *$ & \\
\hline$U * \overline{B S}$ & -0.5575 & 0.1513 & 0.0003 & $* * *$ & \\
\hline$U^{2} * \overline{B S}$ & 0.0083 & 0.0022 & 0.0002 & $* * *$ & \\
\hline$U * \overline{K V}$ & -0.1049 & 0.0201 & $<0.0001$ & $* * * *$ & \\
\hline$U * \overline{K V}^{2}$ & 3.0925 & 0.4220 & $<0.0001$ & $* * * *$ & \\
\hline$U^{2} * \overline{K V}^{2}$ & -0.0460 & 0.0063 & $<0.0001$ & $* * * *$ & \\
\hline$\overline{B S} * B N$ & 0.3346 & 0.1376 & 0.0163 & $*$ & \\
\hline$\overline{K V} * B N$ & -0.4774 & 0.1997 & 0.0182 & $*$ & \\
\hline$U * \overline{B S} * B N$ & -0.0097 & 0.0041 & 0.0186 & $*$ & \\
\hline$U * \overline{K V} * B N$ & 0.0129 & 0.0059 & 0.0308 & $*$ & \\
\hline
\end{tabular}

(a) The model terms were selected by stepwise regression or were added to make the model hierarchical (see Section 5.4.1).

(b) The probability of incorrectly concluding that the coefficient estimate is different from zero. Low values (e.g., $<0.05$ and especially $<0.01$ ) indicate coefficient estimates that are statistically different from zero.

(c) $*=$ p-value $<0.05, * *=$ p-value $<0.01, * * *=p$-value $<0.001$, and $* * * * p$-value $<0.0001$. Also, note that the main (individual) effect of a given parameter may not be statistically significant if that parameter is involved in one or more statistically significant interactions. 

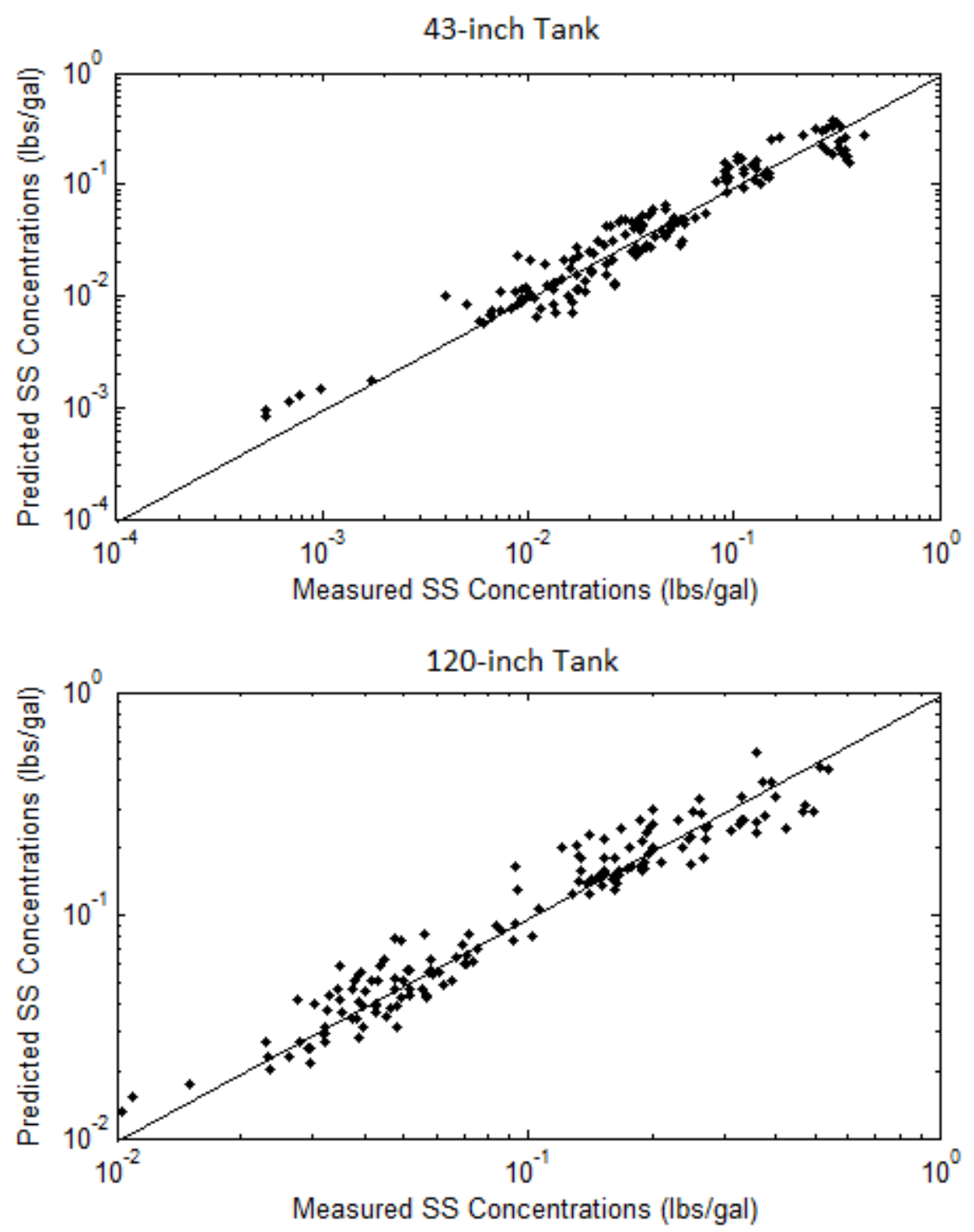

Figure 7.2. Predicted versus Measured Plots for the Stainless Steel Concentration Models from Table 7.2 for the 43-inch Tank and 120-inch Tank (For Information Only)

\subsubsection{Models for Sand Concentrations as Functions of the Test Parameters with Up to a Cubic Polynomial Dependence on Jet Velocity}

Table 7.3 presents the model terms, estimated coefficients, coefficient standard deviations, and coefficient p-values for the models of the form in Equation (5.5) obtained by stepwise regression when separately fitting sand concentration data for the 43 -inch and 120-inch tanks. Also included in Table 7.3 are the values of RMSE, $\mathrm{R}^{2}$, and $\mathrm{R}_{\mathrm{A}}^{2}$ for each model, as well as the LOF p-value (see Section 5.4.2). The model for the 43-inch tank was fitted after eliminating one observation (corresponding to the pre-transfer sample for Test Combination 18) due to its outlying and influential nature (see Figure B.3 and Figure B.7). No observations were eliminated in fitting the model to the data for the 120 -inch tank. 
Table 7.3 shows that the $\ln \left(C_{\text {sand }}\right)$ models for the 43 -inch and 120 -inch tanks perform well (both have $\mathrm{R}^{2}$ values above 0.95 ) and do not have statistically significant LOFs ( $\mathrm{p}$-values $=0.9999$ and 0.6778 ).

Figure 7.3 displays the PvM plots (see Section 5.4.2) corresponding to the sand concentration models in Table 7.3 for the 43 -inch and 120 -inch tanks. Figure 7.3 shows the measured sand concentration data separated in two clusters corresponding to the two different base stimulants used. The model for the 43-inch tank predicts sand concentration accurately for the entire range of experimentally observed values, with the exception of appearing to overpredict measured values in the range $0.45-0.65 \mathrm{lb} / \mathrm{gal}$. The model for the 120-inch tank shows a tendency to underpredict in some ranges of measured sand concentrations $(0.22-0.28$ and $>0.89 \mathrm{lb} /$ gal, approximately) and overpredict in other ranges $(<0.17$ and $0.40-0.65 \mathrm{lb} /$ gal, approximately).

Table 7.3. Model Terms, Estimated Coefficients, Coefficient Standard Deviations, Coefficient p-Values, and Model Summary Statistics for the Sand Concentration Models, Corresponding to the 43-inch and 120-inch Tanks, of the Form in Equation (5.5) Obtained by Stepwise Regression

\begin{tabular}{|c|c|c|c|c|c|}
\hline $\begin{array}{c}\ln \left(C_{\text {Sand }}\right) \\
\text { Model Term }^{(a)}\end{array}$ & $\begin{array}{l}\text { Coefficient } \\
\text { Estimate }\end{array}$ & SD(Coefficient) & p-value ${ }^{(b)}$ & Significance $^{(c)}$ & $\begin{array}{c}\text { Model Fit Summary } \\
\text { Statistics }\end{array}$ \\
\hline \multicolumn{6}{|c|}{ 43-inch Tank } \\
\hline Intercept & -3.6960 & 0.2234 & $<0.0001$ & $* * * *$ & $\mathrm{RMSE}=0.1087$ \\
\hline$U$ & 0.1735 & 0.0168 & $<0.0001$ & $* * * *$ & $\mathrm{R}^{2}=0.9784$ \\
\hline$\overline{B S}$ & 2.0968 & 0.2231 & $<0.0001$ & $* * * *$ & $\mathrm{R}_{\mathrm{A}}^{2}=0.9771$ \\
\hline$\overline{K V}$ & -0.0043 & 0.0301 & 0.8861 & & LOF $p$-value $=0.9999$ \\
\hline$B N$ & -0.0032 & 0.0062 & 0.6130 & & \\
\hline$U^{2}$ & -0.0026 & 0.0003 & $<0.0001$ & $* * * *$ & \\
\hline$U * \overline{B S}$ & -0.0972 & 0.0168 & $<0.0001$ & $* * * *$ & \\
\hline$U^{2} * \overline{B S}$ & 0.0017 & 0.0003 & $<0.0001$ & $* * * *$ & \\
\hline$\overline{B S} * B N$ & -0.0199 & 0.0054 & 0.0003 & $* * *$ & \\
\hline$\overline{K V} * B N$ & 0.0233 & 0.0099 & 0.0201 & $*$ & \\
\hline \multicolumn{6}{|c|}{ 120-inch Tank } \\
\hline Intercept & -1.1622 & 0.1296 & $<0.0001$ & $* * * *$ & $\mathrm{RMSE}=0.1550$ \\
\hline$U$ & 0.0077 & 0.0038 & 0.0423 & $*$ & $\mathrm{R}^{2}=0.9551$ \\
\hline$\overline{B S}$ & 0.7650 & 0.0236 & $<0.0001$ & $* * * *$ & $\mathrm{R}_{\mathrm{A}}^{2}=0.9530$ \\
\hline$\overline{K V}$ & -0.0857 & 0.0434 & 0.0500 & $*$ & LOF $p$-value $=0.6778$ \\
\hline$\overline{C V}$ & 0.0908 & 0.0205 & $<0.0001$ & $* * * *$ & \\
\hline$B N$ & -0.0148 & 0.0082 & 0.0728 & & \\
\hline$\overline{B S} * B N$ & -0.0320 & 0.0077 & 0.0001 & $* * *$ & \\
\hline$\overline{K V} * B N$ & 0.0353 & 0.0130 & 0.0074 & $* *$ & \\
\hline \multicolumn{6}{|c|}{$\begin{array}{l}\text { (a) The model terms were selected by stepwise regression or were added to make the model hierarchical (see } \\
\text { Section } 5.4 .1 \text { ). } \\
\text { (b) The probability of incorrectly concluding that the coefficient estimate is different from zero. Low values (e.g., } \\
<0.05 \text { and especially }<0.01 \text { ) indicate coefficient estimates that are statistically different from zero. } \\
\text { (c) } *=\text { p-value }<0.05, * *=p \text {-value }<0.01, * * *=p \text {-value }<0.001 \text {, and } * * * *=p \text {-value }<0.0001 \text {. Also, note that } \\
\text { the main (individual) effect of a given parameter may not be statistically significant if that parameter is } \\
\text { involved in one or more statistically significant interactions. }\end{array}$} \\
\hline
\end{tabular}



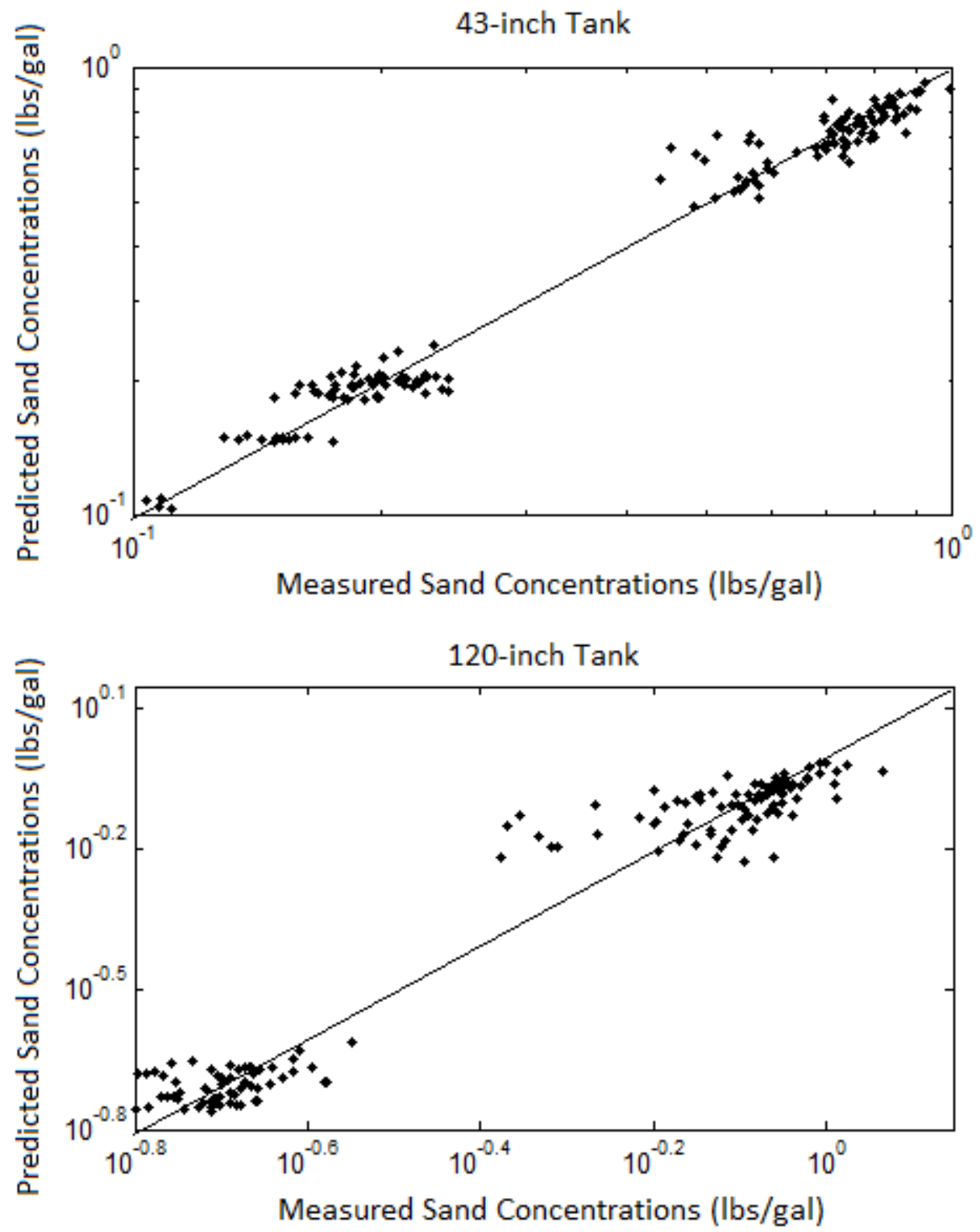

Figure 7.3. Predicted versus Measured Plots for the Sand Concentration Models from Table 7.3 for the 43-inch Tank and 120-inch Tank (For Information Only)

\subsubsection{Models for $\mathrm{ZrO}_{2}$ Concentrations as Functions of the Test Parameters with Up to a Cubic Polynomial Dependence on Jet Velocity}

Table 7.4 presents the model terms, estimated coefficients, coefficient standard deviations, and coefficient p-values for the models of the form in Equation (5.5) obtained by stepwise regression when separately fitting $\mathrm{ZrO}_{2}$ concentration data for the 43-inch and 120-inch tanks. Also included in Table 7.4 are the values of RMSE, $\mathrm{R}^{2}$, and $\mathrm{R}_{\mathrm{A}}^{2}$ for each model, as well as the LOF p-value (see Section 5.4.2). Table 7.4 indicates that the $\ln \left(C_{\mathrm{ZrO} 2}\right)$ models for the 43 -inch and 120 -inch tanks have $\mathrm{R}^{2}$ values of 0.8151 and 0.6906 , respectively. Hence, there are substantive fractions of the data variability that were not accounted for by the models, which seems surprising considering that both models contain a relatively large number of terms. However, the models do not have statistically significant LOF ( $p$-values $=0.5424$ 
and 0.3400 ). Thus, the variation in the data sets for the 43 -inch and 120-inch tanks is not accounted for by the models in Table 7.4. The variation in the data sets is a result of experimental testing and measurement uncertainty and hence cannot be accounted for by any model.

Table 7.4. Model Terms, Estimated Coefficients, Coefficient Standard Deviations, Coefficient p-Values, and Model Summary Statistics for the $\mathrm{ZrO}_{2}$ Concentration Models, Corresponding to the 43-inch and 120-inch Tanks, of the Form in Equation (5.5) Obtained by Stepwise Regression

\begin{tabular}{|c|c|c|c|c|c|}
\hline $\begin{array}{c}\ln \left(\mathrm{C}_{\mathrm{ZrO} 2}\right) \\
\text { Model Term }^{(\mathrm{a})}\end{array}$ & $\begin{array}{l}\text { Coefficient } \\
\text { Estimate }\end{array}$ & SD(Coefficient) & p-value ${ }^{(b)}$ & Significance $^{(c)}$ & $\begin{array}{c}\text { Model Fit Summary } \\
\text { Statistics }\end{array}$ \\
\hline \multicolumn{6}{|c|}{ 43-inch Tank } \\
\hline Intercept & -5.9318 & 1.6155 & 0.0003 & $* * *$ & $\mathrm{RMSE}=0.1014$ \\
\hline$U$ & 0.4660 & 0.1950 & 0.0182 & $*$ & $\mathrm{R}^{2}=0.8151$ \\
\hline$\overline{B S}$ & 0.7608 & 0.2315 & 0.0013 & $* *$ & $\mathrm{R}_{\mathrm{A}}^{2}=0.7953$ \\
\hline$\overline{K V}$ & 1.7779 & 0.5524 & 0.0016 & $* *$ & LOF $p$-value $=0.5424$ \\
\hline$\overline{C V}$ & 0.0442 & 0.0148 & 0.0033 & $* *$ & \\
\hline$B N$ & -0.0013 & 0.0048 & 0.7911 & & \\
\hline$U^{2}$ & -0.0188 & 0.0076 & 0.0149 & $*$ & \\
\hline$U^{3}$ & 0.0002 & 0.0001 & 0.0112 & $*$ & \\
\hline$\overline{K V}^{2}$ & -0.4486 & 0.2210 & 0.0442 & $*$ & \\
\hline$U * \overline{B S}$ & -0.0645 & 0.0179 & 0.0004 & $* * *$ & \\
\hline$U^{2} * \overline{B S}$ & 0.0011 & 0.0003 & 0.0013 & $* *$ & \\
\hline$\overline{B S} * \overline{K V}$ & 0.0676 & 0.0202 & 0.0011 & $* *$ & \\
\hline$U * \overline{K V}$ & -0.1537 & 0.0453 & 0.0009 & $* * *$ & \\
\hline$U * \overline{K V}^{2}$ & 0.0197 & 0.0085 & 0.0214 & $*$ & \\
\hline$U^{2} * \overline{K V}$ & 0.0033 & 0.0009 & 0.0006 & $* * *$ & \\
\hline$\overline{B S} * B N$ & -0.0097 & 0.0048 & 0.0045 & $*$ & \\
\hline $\begin{array}{l}\text { (a) The model term } \\
\text { Section 5.4.1). } \\
\text { (b) The probability } \\
<0.05 \text { and espe } \\
\text { (c) } *=\text { p-value }<0 . \\
\text { the main (indivi } \\
\text { involved in one }\end{array}$ & $\begin{array}{l}\text { re selected by } \\
\text { ncorrectly con } \\
y<0.01) \text { indi } \\
* *=p \text {-value } \\
\text { l) effect of a } g \\
\text { nore statistica }\end{array}$ & $\begin{array}{l}\text { stepwise regression } \\
\text { luding that the coef } \\
\text { ate coefficient estin } \\
0.01, * * *=p \text {-value } \\
\text { ren parameter may } \\
\text { y significant interac }\end{array}$ & $\begin{array}{l}\text { or were adde } \\
\text { icient estime } \\
\text { ates that are } \\
<0.001 \text {, and } \\
\text { lot be statisti } \\
\text { ions. }\end{array}$ & $\begin{array}{l}\mathrm{d} \text { to make the mo } \\
\text { te is different fro } \\
\text { tatistically differ } \\
* * * *=\mathrm{p} \text {-value }< \\
\text { ally significant } \mathrm{i}\end{array}$ & $\begin{array}{l}\text { del hierarchical (see } \\
\text { m zero. Low values (e.g., } \\
\text { ent from zero. } \\
0.0001 \text {. Also, note that } \\
\text { f that parameter is }\end{array}$ \\
\hline
\end{tabular}


Table 7.4. Model Terms, Estimated Coefficients, Coefficient Standard Deviations, Coefficient p-Values, and Model Summary Statistics for the $\mathrm{ZrO}_{2}$ Concentration Models, Corresponding to the 43-inch and 120-inch Tanks, of the Form in Equation (5.5) Obtained by Stepwise Regression (cont.)

\begin{tabular}{|c|c|c|c|c|c|}
\hline $\begin{array}{c}\ln \left(\mathrm{C}_{\mathrm{ZrO2}}\right) \\
\text { Model Term }^{(\mathrm{a})}\end{array}$ & $\begin{array}{l}\text { Coefficient } \\
\text { Estimate }\end{array}$ & SD(Coefficient) & p-value ${ }^{(\mathbf{b})}$ & Significance ${ }^{(c)}$ & $\begin{array}{c}\text { Model Fit Summary } \\
\text { Statistics }\end{array}$ \\
\hline \multicolumn{6}{|c|}{ 120-inch Tank } \\
\hline Intercept & -31.1061 & 12.6513 & 0.0151 & $*$ & $\mathrm{RMSE}=0.1324$ \\
\hline$U$ & 2.5918 & 1.1148 & 0.0215 & $*$ & $\mathrm{R}^{2}=0.6906$ \\
\hline$\overline{B S}$ & 3.9881 & 1.3387 & 0.0034 & $* *$ & $\mathrm{R}_{\mathrm{A}}^{2}=0.6670$ \\
\hline$\overline{K V}$ & -0.0288 & 0.0284 & 0.3119 & & LOF $p$-value $=0.3400$ \\
\hline$\overline{C V}$ & 0.0954 & 0.0179 & $<0.0001$ & $* * * *$ & \\
\hline$U^{2}$ & -0.0764 & 0.0326 & 0.0203 & $*$ & \\
\hline$U^{3}$ & 0.0007 & 0.0003 & 0.0199 & $*$ & \\
\hline$\overline{K V}^{2}$ & -0.8180 & 0.3520 & 0.0215 & $*$ & \\
\hline$U * \overline{B S}$ & -0.2541 & 0.0790 & 0.0016 & $* *$ & \\
\hline$U^{2} * \overline{B S}$ & 0.0038 & 0.0012 & 0.0012 & $* *$ & \\
\hline$\overline{B S} * \overline{K V}$ & -0.0476 & 0.0226 & 0.0372 & $*$ & \\
\hline$U * \overline{K V}^{2}$ & 0.0279 & 0.0105 & 0.0089 & $* *$ & \\
\hline \multicolumn{6}{|c|}{$\begin{array}{l}\text { (a) The model terms were selected by stepwise regression or were added to make the model hierarchical (see } \\
\text { Section 5.4.1). } \\
\text { (b) The probability of incorrectly concluding that the coefficient estimate is different from zero. Low values (e.g., } \\
<0.05 \text { and especially }<0.01 \text { ) indicate coefficient estimates that are statistically different from zero. } \\
\text { (c) } *=\text { p-value }<0.05, * *=\text { p-value }<0.01, * * *=\text { p-value }<0.001 \text {, and } * * * *=\text { p-value }<0.0001 \text {. Also, note that } \\
\text { the main (individual) effect of a given parameter may not be statistically significant if that parameter is } \\
\text { involved in one or more statistically significant interactions. }\end{array}$} \\
\hline
\end{tabular}

Figure 7.4 displays the PvM plots (see Section 5.4.2) corresponding to the $\mathrm{ZrO}_{2}$ concentration models in Table 7.4 for the 43 -inch and 120 -inch tanks.

- The top panel of Figure 7.4 (corresponding to the model for the 43-inch tank) shows two clusters of points corresponding to the two base simulants having distributions much narrower in the vertical direction (predicted $\mathrm{ZrO}_{2}$ concentration values) than in the horizontal direction (measured $\mathrm{ZrO}_{2}$ concentration values). These distributions of points in each cluster thus cause a tendency to overpredict $\mathrm{ZrO}_{2}$ concentrations on the left side of each cluster and underpredict $\mathrm{ZrO}_{2}$ concentrations on the right side of each cluster.

- The bottom panel of Figure 7.4 (corresponding to the model for the 120-inch tank) again shows two clusters of points. The cluster with the higher measured $\mathrm{ZrO}_{2}$ concentrations has similar vertical and horizontal distributions. On the other hand, the cluster with the lower measured $\mathrm{ZrO}_{2}$ concentrations spans about three-quarters of the range of measured $\mathrm{ZrO}_{2}$ concentrations. This causes a tendency to overpredict $\mathrm{ZrO}_{2}$ concentrations on the left side of this cluster and underpredict on the right side of the cluster. 

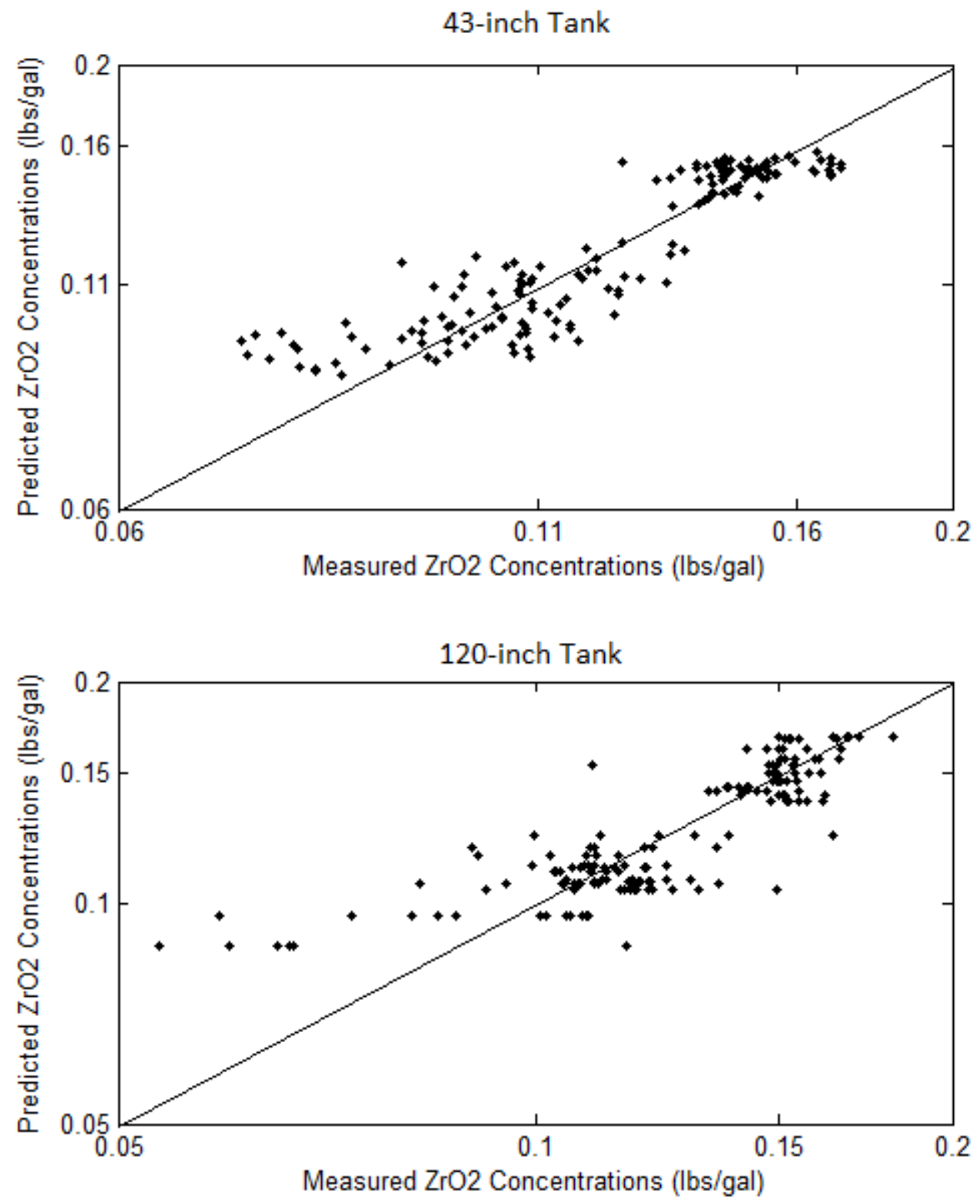

Figure 7.4. Predicted versus Measured Plots for the $\mathrm{ZrO}_{2}$ Concentration Models from Table 7.4 for the 43-inch Tank and 120-inch Tank (For Information Only) 


\subsection{Results of Fitting Models for Component Concentrations as Functions of the Test Parameters with a Linearization and Quadratic Extension of a Power-Law Dependence on Jet Velocity}

Sections 7.2.1 through 7.2.4 present the results of applying stepwise regression to obtain reductions of the model in Equation (5.8) for concentrations of gibbsite, SS, sand, and $\mathrm{ZrO}_{2}$, respectively. For each component, separate models were fit to data from the 43-inch tank and the 120-inch tank.

\subsubsection{Models for Gibbsite Concentrations as Functions of the Test Parameters with a Linearization and Quadratic Extension of a Power-Law Dependence on Jet Velocity}

Table 7.5 presents the model terms, estimated coefficients, coefficient standard deviations, and coefficient p-values for the models of the form in Equation (5.8) obtained by stepwise regression when separately fitting gibbsite concentration data for the 43-inch and 120-inch tanks. Also included in Table

7.5 are the values of RMSE, $\mathrm{R}^{2}$, and $\mathrm{R}_{\mathrm{A}}^{2}$ for each model, as well as the LOF p-value (see Section 5.4.2). Before these results are discussed, note that the model for the 120-inch tank in Table 7.5 is exactly the same as the model for the 120 -inch tank in Table 7.1. There were no terms involving $U$ in the model for the 120-inch tank in Table 7.1, and there are no terms involving $\ln (U)$ in the model for the 120-inch tank in Table 7.5. Hence, the same model for the 120-inch tank resulted from stepwise regression even though there was the potential for the models to be different in Tables 7.1 and 7.5.

Table 7.5 shows that the $\ln \left(C_{\text {gibbsite }}\right)$ models for the 43-inch and 120-inch tanks account for the vast majority of the variation in the data (both $\mathrm{R}^{2}$ values are greater than 0.99 ) and neither model has a statistically significant LOF (p-values $=0.8018$ and 0.3430$)$. Finally, note that the $\ln \left(C_{\text {gibssite }}\right)$ model for the 43-inch tank depends on $\ln (U)$, but the model for the 120 -inch tank does not depend on $\ln (U)$. Hence, it is not possible to use the EP approach described in Section 6.2 to calculate scaling exponents for gibbsite concentrations using the models in Table 7.5.

Figure 7.5 displays the PvM plots (see Section 5.4.2) corresponding to the gibbsite concentration models in Table 7.5 for the 43-inch and 120-inch tanks. Both panels in Figure 7.5 show two clusters of points, because of the very different gibbsite concentrations in the two base simulants (see Table 2.2). The $\ln \left(C_{\text {gibbsite }}\right)$ model for the 43-inch tank contains terms involving $U$ and $\overline{K V}$ in addition to $\overline{B S}$, but the effect of $\overline{B S}$ dominates the effects of $U$ and $\overline{K V}$. The $\ln \left(C_{\text {gibbsite }}\right)$ model for the 120-inch tank contains terms involving $\overline{K V}$ and $\overline{C V}$ in additional to $\overline{B S}$, but the effect of $\overline{B S}$ dominates the effects of $\overline{K V}$ and $\overline{C V}$. 
Table 7.5. Model Terms, Estimated Coefficients, Coefficient Standard Deviations, Coefficient p-Values, and Model Summary Statistics for the Gibbsite Concentration Models, Corresponding to the 43-inch and 120-inch Tanks, of the Form in Equation (5.8) Obtained by Stepwise Regression

\begin{tabular}{|c|c|c|c|c|c|}
\hline $\begin{array}{c}\ln \left(C_{\text {gibbsite }}\right) \\
\text { Model Term }^{(a)}\end{array}$ & $\begin{array}{l}\text { Coefficient } \\
\text { Estimate }\end{array}$ & SD(Coefficient) & p-value ${ }^{(b)}$ & Significance $^{(c)}$ & $\begin{array}{c}\text { Model Fit Summary } \\
\text { Statistics }\end{array}$ \\
\hline \multicolumn{6}{|c|}{ 43-inch Tank } \\
\hline Intercept & -1.8850 & 0.2028 & $<0.0001$ & $* * * *$ & $\mathrm{RMSE}=0.1204$ \\
\hline $\ln (U)$ & 0.0969 & 0.0625 & 0.1232 & & $\mathrm{R}^{2}=0.9946$ \\
\hline$\overline{B S}$ & -1.1521 & 0.1766 & $<0.0001$ & $* * * *$ & $\mathrm{R}_{\mathrm{A}}^{2}=0.9944$ \\
\hline$\overline{K V}$ & -0.6675 & 0.3394 & 0.0511 & & LOF $p$-value $=0.8018$ \\
\hline $\ln (U) * \overline{B S}$ & -0.1392 & 0.0541 & 0.0111 & $*$ & \\
\hline $\ln (U) * \overline{K V}$ & 0.2155 & 0.1043 & 0.0406 & $*$ & \\
\hline$\overline{K V}^{2}$ & 0.0758 & 0.0319 & 0.0186 & $*$ & \\
\hline \multicolumn{6}{|c|}{ 120-inch Tank } \\
\hline Intercept & -1.5973 & 0.0187 & $<0.0001$ & $* * * *$ & $\mathrm{RMSE}=0.1491$ \\
\hline$\overline{B S}$ & -1.5920 & 0.0128 & $<0.0001$ & $* * * *$ & $\mathrm{R}^{2}=0.9916$ \\
\hline$\overline{K V}$ & -0.0752 & 0.0283 & 0.0087 & $* *$ & $\mathrm{R}_{\mathrm{A}}^{2}=0.9914$ \\
\hline$\overline{C V}$ & 0.1056 & 0.0197 & $<0.0001$ & $*$ & LOF $p$-value $=0.3430$ \\
\hline$\overline{K V}^{2}$ & 0.0931 & 0.0328 & 0.0051 & $* *$ & \\
\hline
\end{tabular}

(a) The model terms were selected by stepwise regression or were added to make the model hierarchical (see Section 5.4.1).

(b) The probability of incorrectly concluding that the coefficient estimate is different from zero. Low values (e.g., $<0.05$ and especially $<0.01$ ) indicate coefficient estimates that are statistically different from zero.

(c) $*=$ p-value $<0.05, * *=$ p-value $<0.01,{ }^{* * *}=\mathrm{p}$-value $<0.001$, and $* * * *=\mathrm{p}$-value $<0.0001$. Also, note that the main (individual) effect of a given parameter may not be statistically significant if that parameter is involved in one or more statistically significant interactions. 

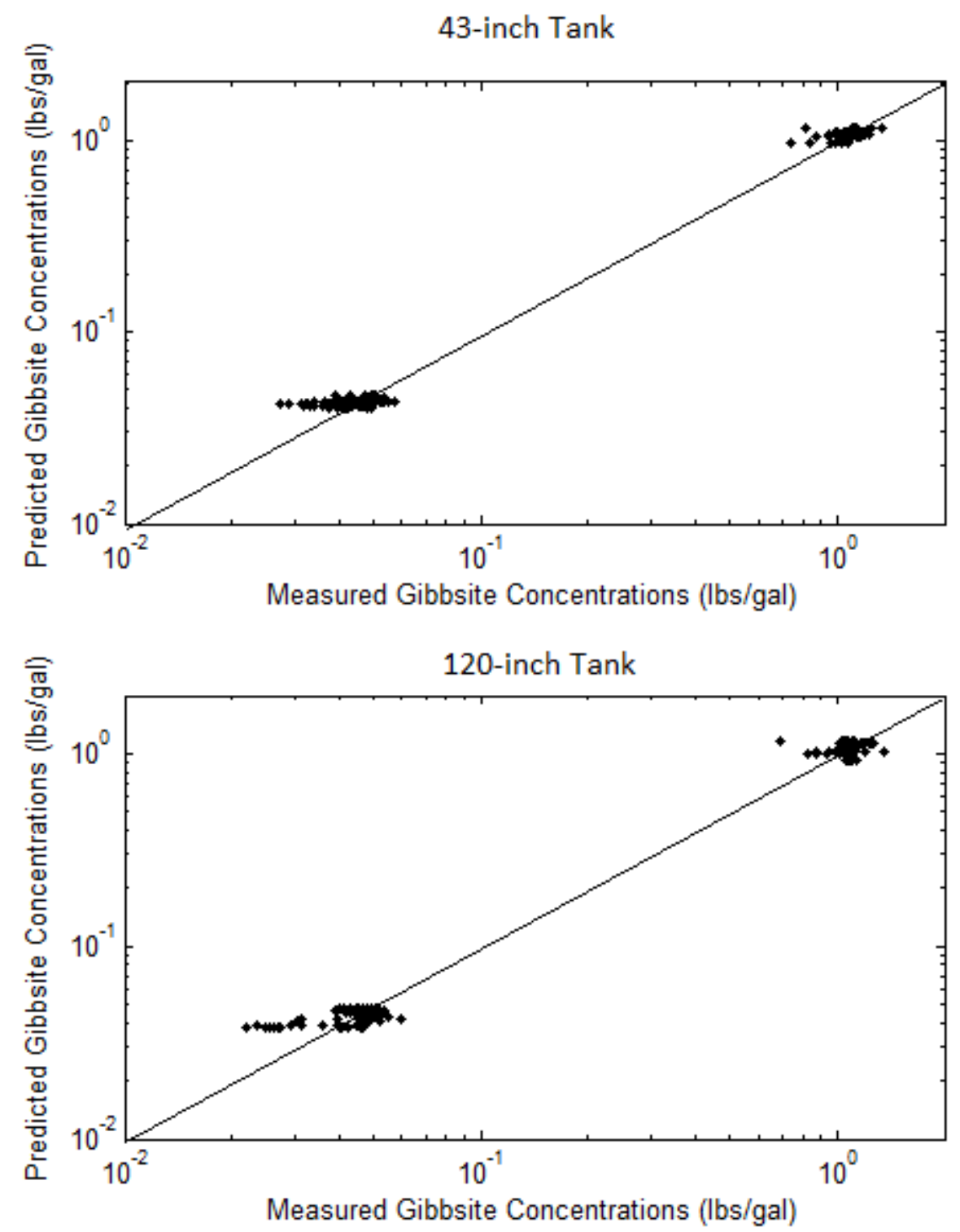

Figure 7.5. Predicted versus Measured Plots for the Gibbsite Concentration Models from Table 7.5 for the 43-inch Tanks and 120-inch Tank (For Information Only)

\subsubsection{Models for Stainless Steel Concentrations as Functions of the Test Parameters with a Linearization and Quadratic Extension of a Power-Law Dependence on Jet Velocity}

Table 7.6 presents the model terms, estimated coefficients, coefficient standard deviations, and coefficient p-values for the models of the form in Equation (5.8) obtained by stepwise regression when separately fitting SS concentration data for the 43-inch and 120-inch tanks. Also included in Table 7.6 are the values of RMSE, $\mathrm{R}^{2}$, and $\mathrm{R}_{\mathrm{A}}^{2}$ for each model, as well as the LOF p-value (see Section 5.4.2). The model for the 43-inch tank was fitted after eliminating two observations corresponding to Batch 1 for Test Combination 22 and Batch 5 for Test Combination 18 because of their outlying and influential nature (see Figure B.6). 
Table 7.6 shows that the $\ln \left(C_{S S}\right)$ models for the 43 -inch and 120 -inch tanks have a relatively large number of terms, with both models depending on $U$. The model for the 43-inch tank accounts for a relatively high fraction of the variation in the $\ln \left(C_{S S}\right)$ values $\left(\mathrm{R}^{2}=0.9350\right)$ and does not have a statistically significant LOF ( $\mathrm{p}$-values $=0.7482)$. The model for the 120 -inch tank accounts for a slightly smaller fraction of the variation in the $\ln \left(C_{S S}\right)$ values $\left(\mathrm{R}^{2}=0.9074\right)$ and has a statistically significant LOF (p-value $=0.0476)$. This indicates that the model for the 120-inch tank does not account for some variation in $\ln \left(C_{S S}\right)$ beyond what results from replicate tests. However, the $\mathrm{R}^{2}$ value is large enough to use the model for the purposes of this report. The models for SS for the 43-inch and 120-inch tanks derived from Equation (5.5) did not have significant LOF, which should be taken into consideration when choosing models for predicting SS concentration.

Figure 7.6 displays the PvM plots (see Section 5.4.2) corresponding to the SS concentration models in Table 7.6 for the 43-inch and 120-inch tanks. The PvM plot for the 43-inch tank shows that the model may slightly overpredict SS concentrations below $\sim 0.001 \mathrm{lb} / \mathrm{gal}^{(\mathrm{a})}$, but otherwise the model predicts accurately (within the testing uncertainty). The PvM plot for the 120-inch tank shows that the model predicts accurately over the middle of the SS concentration range but has a tendency to (i) overpredict SS concentrations below $0.02 \mathrm{lb} / \mathrm{gal}$ (although there were only three data points with concentrations that low) and (ii) underpredict above $0.40 \mathrm{lb} / \mathrm{gal}$.

\footnotetext{
(a) There were six concentration values below 0.001. Five of these were Batches 1-5 for Test Combination 14 with the 43-inch tank. The sixth was for Batch 1 for Test Combination 22 with the 43 -inch tank. Given that all the batches for Test Combination 14 have very low concentrations, they do not appear to be outliers or unusual. For Test Combination 22, the other batches are also small (around 0.01). Note that this test (Test Combination 22 with the 43-inch tank) had laminar (rather than turbulent) flow conditions and had the worst Reynolds number of all of the tests performed.
} 
Table 7.6. Model Terms, Estimated Coefficients, Coefficient Standard Deviations, Coefficient p-Values, and Model Summary Statistics for the Stainless Steel Concentration Models, Corresponding to the 43-inch and 120-inch Tanks, of the Form in Equation (5.8) Obtained by Stepwise Regression

\begin{tabular}{|c|c|c|c|c|c|}
\hline $\begin{array}{c}\ln \left(\mathrm{C}_{\mathrm{SS}}\right) \\
\text { Model Term }^{(\mathrm{a})}\end{array}$ & $\begin{array}{l}\text { Coefficient } \\
\text { Estimate }\end{array}$ & SD(Coefficient) & p-value ${ }^{(b)}$ & Significance $^{(\mathrm{c})}$ & $\begin{array}{c}\text { Model Fit Summary } \\
\text { Statistics }\end{array}$ \\
\hline \multicolumn{6}{|c|}{ 43-inch Tank } \\
\hline Intercept & -44.6595 & 10.0436 & $<0.0001$ & $* * * *$ & RMSE $=0.3717$ \\
\hline $\ln (U)$ & 20.4040 & 6.2737 & 0.0014 & $* *$ & $\mathrm{R}^{2}=0.9350$ \\
\hline$\overline{B S}$ & 1.0666 & 0.0401 & $<0.0001$ & $* * * *$ & $\mathrm{R}_{\mathrm{A}}^{2}=0.9300$ \\
\hline$\overline{K V}$ & -0.6029 & 0.0883 & $<0.0001$ & $* * * *$ & LOF $p$-value $=0.7482$ \\
\hline$\overline{C V}$ & 0.1898 & 0.053 & 0.0005 & $* * *$ & \\
\hline$B N$ & -0.6818 & 0.2914 & 0.0207 & $*$ & \\
\hline$[\ln (U)]^{2}$ & -2.3625 & 0.9774 & 0.0169 & * & \\
\hline$\overline{K V}^{2}$ & -162.7441 & 20.7842 & $<0.0001$ & $* * * *$ & \\
\hline$\overline{B S} * \overline{K V}$ & 0.2384 & 0.0632 & 0.0002 & $* * *$ & \\
\hline $\ln (U) * \overline{K V}^{2}$ & 100.5721 & 12.7883 & $<0.0001$ & $* * * *$ & \\
\hline$[\ln (U)]^{2} * \overline{K V}^{2}$ & -15.4981 & 1.9625 & $<0.0001$ & $* * * *$ & \\
\hline $\ln (U) * B N$ & 0.1818 & 0.0892 & 0.0434 & $*$ & \\
\hline \multicolumn{6}{|c|}{ 120-inch Tank } \\
\hline Intercept & -91.8515 & 31.2626 & 0.0038 & $* *$ & $\mathrm{RMSE}=0.2813$ \\
\hline $\ln (U)$ & 47.6086 & 17.8242 & 0.0084 & $* *$ & $\mathrm{R}^{2}=0.9074$ \\
\hline$\overline{B S}$ & 0.8170 & 0.0249 & $<0.0001$ & $* * * *$ & $\mathrm{R}_{\mathrm{A}}^{2}=0.9023$ \\
\hline$\overline{K V}$ & 193.1144 & 43.1314 & $<0.0001$ & $* * * *$ & LOF $p$-value $=0.0476$ \\
\hline$\overline{C V}$ & 0.1056 & 0.0375 & 0.0056 & $* *$ & \\
\hline$B N$ & -0.0949 & 0.0133 & $<0.0001$ & $* * * *$ & \\
\hline $\ln (U) * \overline{K V}$ & -109.4058 & 24.595 & $<0.0001$ & $* * * *$ & \\
\hline$[\ln (U)]^{2}$ & -6.2841 & 2.5391 & 0.0145 & * & \\
\hline$[\ln (U)]^{2} * \overline{K V}$ & 15.4799 & 3.5038 & $<0.0001$ & $* * * *$ & \\
\hline
\end{tabular}

(a) The model terms were selected by stepwise regression or were added to make the model hierarchical (see Section 5.4.1).

(b) The probability of incorrectly concluding that the coefficient estimate is different from zero. Low values (e.g., $<0.05$ and especially $<0.01)$ indicate coefficient estimates that are statistically different from zero.

(c) $*=$ p-value $<0.05, * *=$ p-value $<0.01, * * *=$ p-value $<0.001$, and $* * * *=p$-value $<0.0001$. Also, note that the main (individual) effect of a given parameter may not be statistically significant if that parameter is involved in one or more statistically significant interactions. 

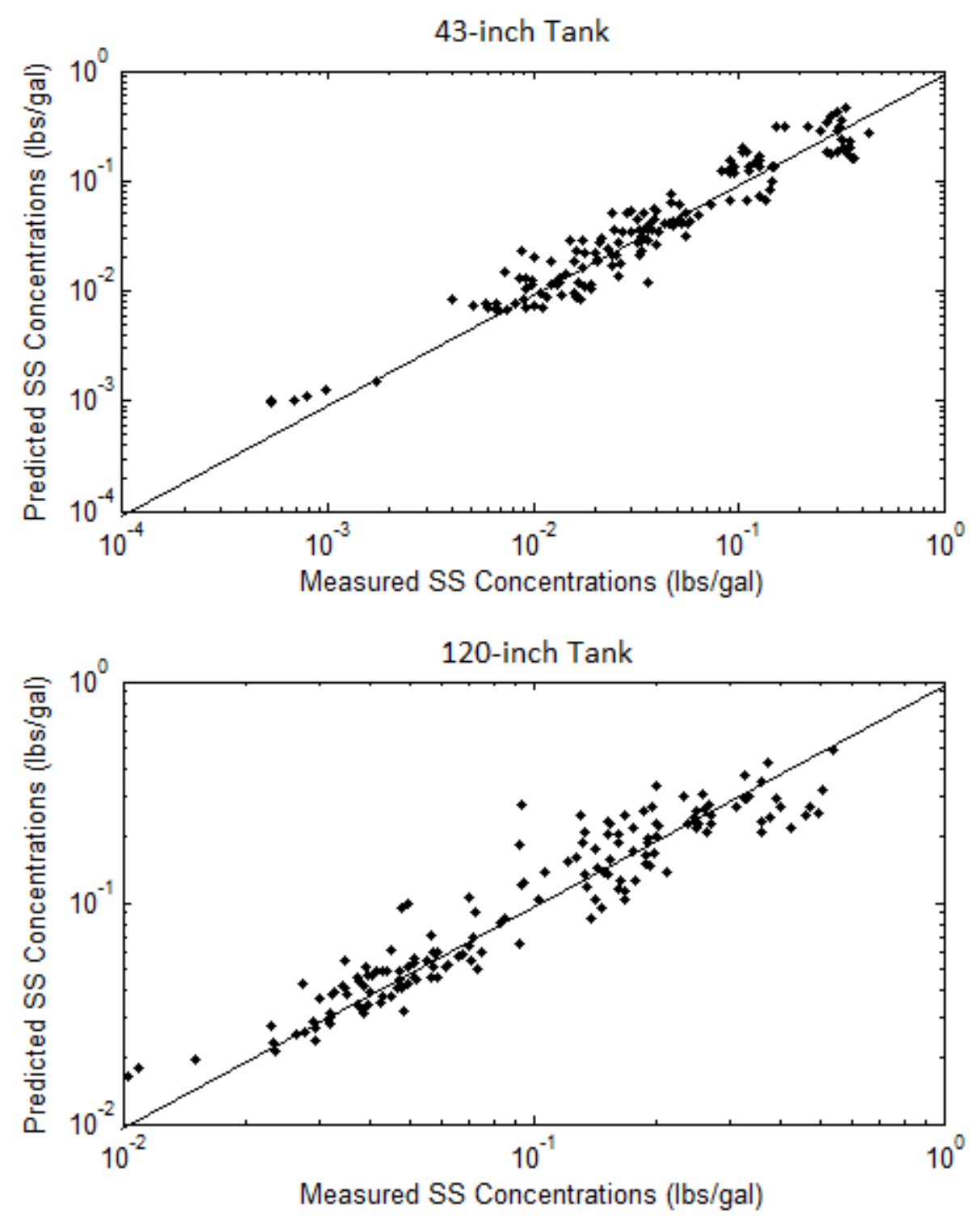

Figure 7.6. Predicted versus Measured Plots for the Stainless Steel Concentration Models from Table 7.6 for the 43-inch Tank and 120-inch Tank (For Information Only)

\subsubsection{Models for Sand Concentrations as Functions of the Test Parameters with a Linearization and Quadratic Extension of a Power-Law Dependence on Jet Velocity}

Table 7.7 presents the model terms, estimated coefficients, coefficient standard deviations, and coefficient p-values for the models of the form in Equation (5.8) obtained by stepwise regression when separately fitting sand concentration data for the 43-inch and 120-inch tanks. Also included in Table 7.7 are the values of RMSE, $\mathrm{R}^{2}$, and $\mathrm{R}_{\mathrm{A}}^{2}$ for each model, as well as the LOF p-value (see Section 5.4.2). The model for the 43-inch tank was fitted after eliminating one observation (corresponding to the pre-transfer sample for Test Combination 18) due to its outlying and influential nature (see Figure B.3 and Figure B.7). No observations were eliminated in fitting the model to the data for the 120 -inch tank. 
Table 7.7 shows that the $\ln \left(C_{\text {sand }}\right)$ models for the 43-inch and 120-inch tanks account for most of the variability in the data $\left(\mathrm{R}^{2}=0.9785\right.$ and 0.9552 , respectively). Also, the two models do not have statistically significant LOF ( $\mathrm{p}$-value $=0.9999$ and 0.6791$)$.

Figure 7.7 displays the PvM plots (see Section 5.4.2) corresponding to the sand concentration models in Table 7.7 for the 43 -inch and 120 -inch tanks. Figure 7.7 shows the measured sand concentration data for both tanks are separated in two main clusters corresponding to the two different base stimulants used. For the 43-inch tank data, the main clusters are divided into sub-clusters. For the 120 -inch tank, the main clusters have distributions that are much wider horizontally (measured sand concentrations) than vertically (predicted sand concentrations. This suggests there could be variation within the clusters that is not accounted for by the data. However, the model for the 120 -inch tank does not have a significant LOF, so it is not clear that there is other structure that could be accounted for by different model terms.

Figure 7.7 also shows that the model for the 43 -inch tank predicts sand concentration relatively accurately for the entire range of experimentally observed values, with the exception of possibly overpredicted measured values in the range $0.4-0.6 \mathrm{lb} / \mathrm{gal}$. The model for the 120 -inch tank shows tendencies to overpredict on the left sides of the two main clusters and underpredict on the right sides of the clusters. 
Table 7.7. Model Terms, Estimated Coefficients, Coefficient Standard Deviations, Coefficient p-Values, and Model Summary Statistics for the Sand Concentration Models, Corresponding to the 43-inch and 120-inch Tanks, of the Form in Equation (5.8) Obtained by Stepwise Regression

\begin{tabular}{|c|c|c|c|c|c|}
\hline $\begin{array}{c}\ln \left(C_{\text {Sand }}\right) \\
\text { Model Term }^{(a)}\end{array}$ & $\begin{array}{l}\text { Coefficient } \\
\text { Estimate }\end{array}$ & SD(Coefficient) & p-value ${ }^{(b)}$ & Significance $^{(c)}$ & $\begin{array}{c}\text { Model Fit Summary } \\
\text { Statistics }\end{array}$ \\
\hline \multicolumn{6}{|c|}{ 43-inch Tank } \\
\hline Intercept & -18.3956 & 2.1832 & $<0.0001$ & $* * * *$ & $\mathrm{RMSE}=0.1086$ \\
\hline $\ln (U)$ & 9.9025 & 1.3515 & $<0.0001$ & $* * * *$ & $\mathrm{R}^{2}=0.9785$ \\
\hline$\overline{B S}$ & 13.0435 & 2.1818 & $<0.0001$ & $* * * *$ & $\mathrm{R}_{\mathrm{A}}^{2}=0.9771$ \\
\hline$\overline{K V}$ & 0.0036 & 0.0301 & 0.9051 & & LOF $p$-value $=0.9999$ \\
\hline$B N$ & -0.0032 & 0.0062 & 0.6125 & & \\
\hline $\ln (U) * \overline{B S}$ & -7.4471 & 1.3507 & $<0.0001$ & $* * * *$ & \\
\hline$\overline{B S} * B N$ & -0.0199 & 0.0054 & 0.0003 & $* * *$ & \\
\hline$\overline{K V} * B N$ & 0.0233 & 0.0099 & 0.0201 & $*$ & \\
\hline$[\ln (U)]^{2}$ & -1.3973 & 0.2087 & $<0.0001$ & $* * * *$ & \\
\hline$[\ln (U)]^{2} * \overline{B S}$ & 1.1249 & 0.2086 & $<0.0001$ & $* * * *$ & \\
\hline \multicolumn{6}{|c|}{ 120-inch Tank } \\
\hline Intercept & -1.8419 & 0.4518 & 0.0001 & $* * *$ & $\mathrm{RMSE}=0.1549$ \\
\hline $\ln (U)$ & 0.2676 & 0.1284 & 0.0389 & $*$ & $\mathrm{R}^{2}=0.9552$ \\
\hline$\overline{B S}$ & 0.7651 & 0.0235 & $<0.0001$ & $* * * *$ & $\mathrm{R}_{\mathrm{A}}^{2}=0.9531$ \\
\hline$\overline{K V}$ & -0.0856 & 0.0433 & 0.0500 & $*$ & LOF $p$-value $=0.6791$ \\
\hline$\overline{C V}$ & 0.0909 & 0.0205 & $<0.0001$ & $* * * *$ & \\
\hline$B N$ & -0.0148 & 0.0082 & 0.0727 & & \\
\hline$\overline{B S} * B N$ & -0.0320 & 0.0077 & 0.0001 & $* * *$ & \\
\hline$\overline{K V} * B N$ & 0.0353 & 0.0130 & 0.0074 & $* *$ & \\
\hline
\end{tabular}

(a) The model terms were selected by stepwise regression or were added to make the model hierarchical (see Section 5.4.1).

(b) The probability of incorrectly concluding that the coefficient estimate is different from zero. Low values (e.g., $<0.05$ and especially $<0.01)$ indicate coefficient estimates that are statistically different from zero.

(c) $*=$ p-value $<0.05, * *=$ p-value $<0.01, * * *=p$-value $<0.001$, and $* * * *=p$-value $<0.0001$. Also, note that the main (individual) effect of a given parameter may not be statistically significant if that parameter is involved in one or more statistically significant interactions. 

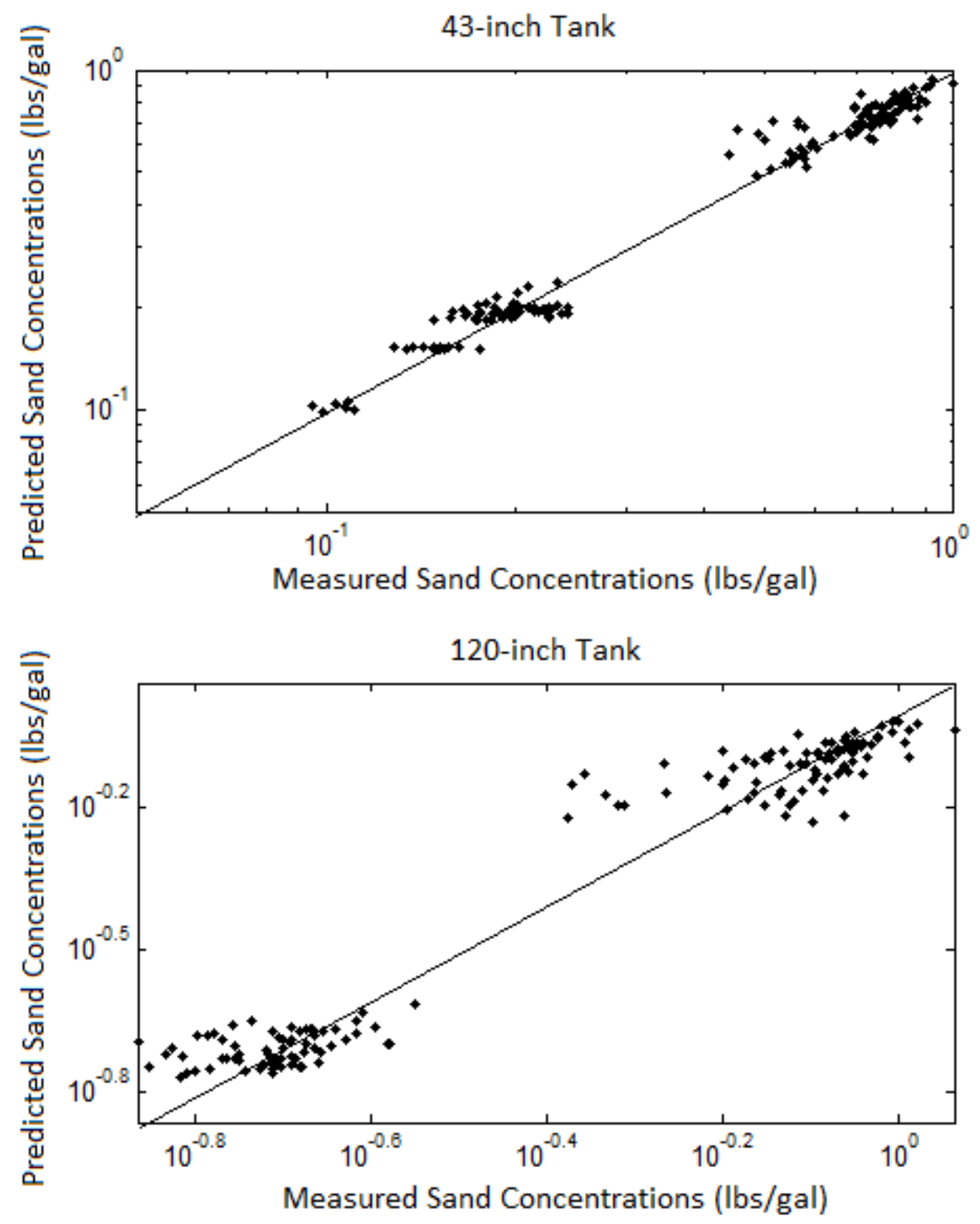

Figure 7.7. Predicted versus Measured Plots for the Sand Concentration Models from Table 7.7 for the 43-inch Tank and 120-inch Tank (For Information Only)

\subsubsection{Models for $\mathrm{ZrO}_{2}$ Concentrations as Functions of the Test Parameters with a Linearization and Quadratic Extension of a Power-Law Dependence on Jet Velocity}

Table 7.8 presents the model terms, estimated coefficients, coefficient standard deviations, and coefficient p-values for the models of the form in Equation (5.8) obtained by stepwise regression when separately fitting $\mathrm{ZrO}_{2}$ concentration data for the 43-inch and 120-inch tanks. Also included in Table 7.8 are the values of RMSE, $\mathrm{R}^{2}$, and $\mathrm{R}_{\mathrm{A}}^{2}$ for each model, as well as the LOF p-value (see Section 5.4.2). Table 7.8 indicates that the $\ln \left(C_{\mathrm{ZrO} 2}\right)$ models for the 43 -inch and 120-inch tanks have $\mathrm{R}^{2}$ values of 0.7652 and 0.6469 , respectively. Hence, there are substantive fractions of the data variability that are not accounted for by the models. However, the models do not have statistically significant LOF ( $p$-values $=$ 
0.3049 and 0.2406 ). Thus, the variation in the data sets for the 43 -inch and 120 -inch tanks not accounted for by the models in Table 7.8 are a result of experimental testing and measurement uncertainty and hence cannot be accounted for by any model. Finally, note that the $\ln \left(C_{\mathrm{ZrO} 2}\right)$ model for the 43 -inch tank depends on $U$, but the model for the 120-inch tank does not depend on $U$. Hence, it is not possible to use the EP approach described in Section 6.2 to calculate scaling exponents for gibbsite concentrations using the models in Table 7.8 .

Table 7.8. Model Terms, Estimated Coefficients, Coefficient Standard Deviations, Coefficient p-Values, and Model Summary Statistics for the $\mathrm{ZrO}_{2}$ Concentration Models, Corresponding to the 43-inch and 120-inch Tanks, of the Form in Equation (5.8) Obtained by Stepwise Regression

\begin{tabular}{|c|c|c|c|c|c|}
\hline $\begin{array}{c}\ln \left(\mathrm{C}_{\mathrm{ZrO} 2}\right) \\
\text { Model Term }^{(\mathrm{a})}\end{array}$ & $\begin{array}{c}\text { Coefficient } \\
\text { Estimate }\end{array}$ & SD(Coefficient) & p-value ${ }^{(b)}$ & Significance $^{(c)}$ & $\begin{array}{c}\text { Model Fit Summary } \\
\text { Statistics }\end{array}$ \\
\hline \multicolumn{6}{|c|}{ 43-inch Tank } \\
\hline Intercept & -2.4729 & 0.1492 & $<0.0001$ & $* * * *$ & $\mathrm{RMSE}=0.1104$ \\
\hline $\ln (U)$ & 0.1117 & 0.0460 & 0.0163 & $*$ & $\mathrm{R}^{2}=0.7652$ \\
\hline$\overline{B S}$ & -0.1691 & 0.0115 & $<0.0001$ & $* * * *$ & $\mathrm{R}_{\mathrm{A}}^{2}=0.7574$ \\
\hline$\overline{K V}$ & 0.0279 & 0.0217 & 0.1991 & & LOF p-value $=0.3049$ \\
\hline$\overline{C V}$ & 0.0328 & 0.0149 & 0.0297 & $*$ & \\
\hline$\overline{\overline{B S}} * \overline{K V}$ & 0.0615 & 0.0174 & 0.0006 & $* * *$ & \\
\hline \multicolumn{6}{|c|}{ 120-inch Tank } \\
\hline Intercept & -2.1208 & 0.0173 & $<0.0001$ & $* * * *$ & $\mathrm{RMSE}=0.1381$ \\
\hline$\overline{\overline{B S}}$ & -0.1702 & 0.0118 & $<0.0001$ & $* * * *$ & $\mathrm{R}^{2}=0.6469$ \\
\hline$\overline{K V}$ & -0.0443 & 0.0262 & 0.0934 & & $\mathrm{R}_{\mathrm{A}}^{2}=0.6376$ \\
\hline$\overline{C V}$ & 0.0931 & 0.0182 & $<0.0001$ & $* * * *$ & LOF $p$-value $=0.2406$ \\
\hline$\overline{K V}^{2}$ & 0.0755 & 0.0304 & 0.0140 & * & \\
\hline
\end{tabular}

(a) The model terms were selected by stepwise regression or were added to make the model hierarchical (see Section 5.4.1).

(b) The probability of incorrectly concluding that the coefficient estimate is different from zero. Low values (e.g., $<0.05$ and especially $<0.01)$ indicate coefficient estimates that are statistically different from zero.

(c) $*=$ p-value $<0.05, * *=$ p-value $<0.01,{ }^{* * *}=\mathrm{p}$-value $<0.001$, and $* * * *=\mathrm{p}$-value $<0.0001$. Also, note that the main (individual) effect of a given parameter may not be statistically significant if that parameter is involved in one or more statistically significant interactions.

Figure 7.8 displays the PvM plots (see Section 5.4.2) corresponding to the $\mathrm{ZrO}_{2}$ concentration models in Table 7.8 for the 43-inch and 120-inch tanks. The PvM plots for both models show two clusters of points (corresponding to the two base simulants) that have distributions that are much narrower in the vertical direction (predicted $\mathrm{ZrO}_{2}$ concentration values) than in the horizontal direction (measured $\mathrm{ZrO}_{2}$ concentration values). These distributions of points in each cluster thus cause a tendency to overpredict $\mathrm{ZrO}_{2}$ concentrations on the left side of each cluster and underpredict $\mathrm{ZrO}_{2}$ concentrations on the right side of each cluster. The tendency to overpredict on the left side of the clusters is higher for the 120-inch tank model, especially for the cluster with lower concentrations $(B S=$ High $)$. 

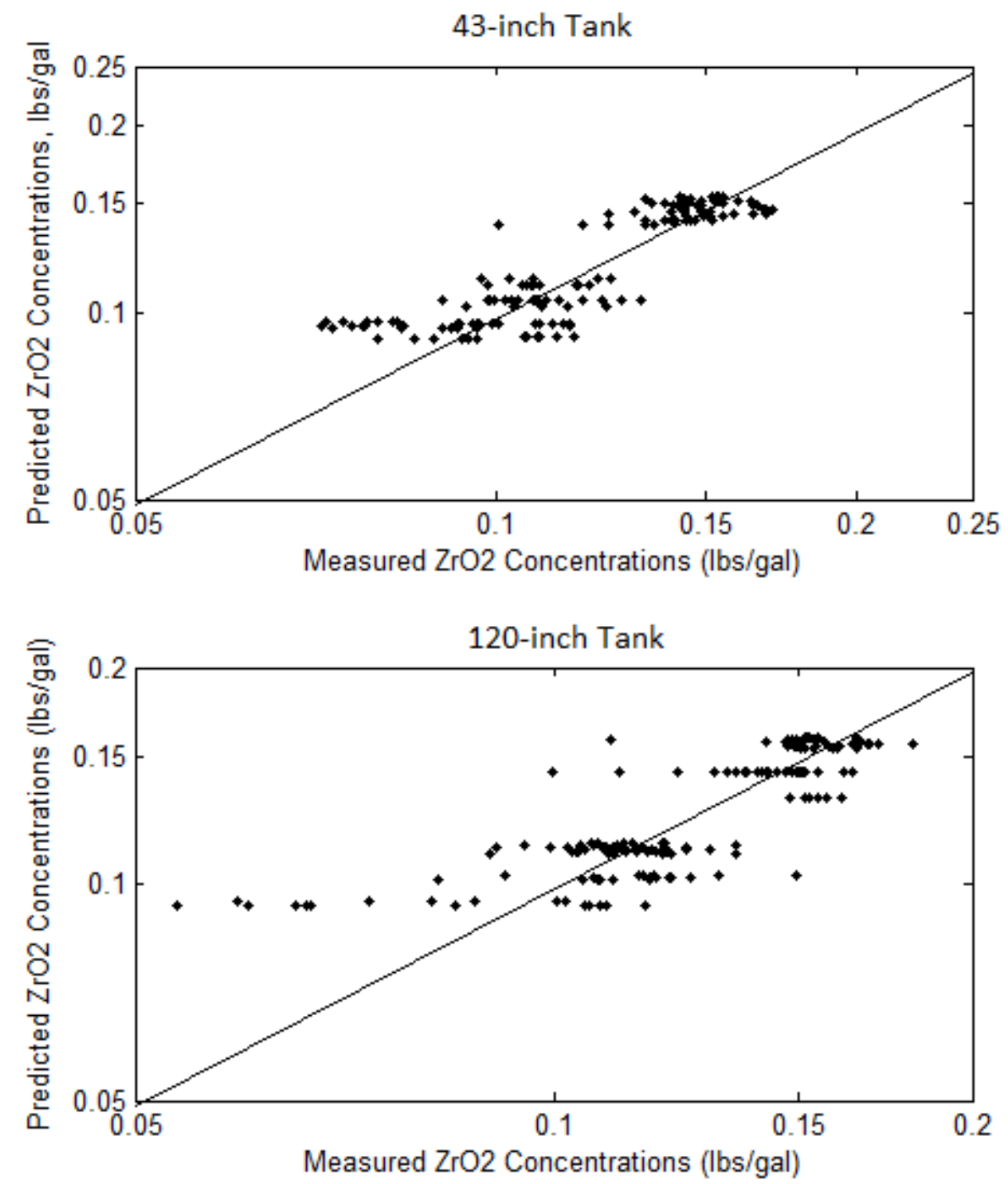

Figure 7.8. Predicted versus Measured Plots for the $\mathrm{ZrO}_{2}$ Concentration Models from Table 7.8 for the 43-inch Tank and 120-inch Tank (For Information Only)

\subsection{Comparisons of Fitted Models from the Two Approaches}

Table 7.9 summarizes the model terms, $\mathrm{R}^{2}$ values, and model LOF results for each of the component concentration models in Sections 7.1 and 7.2 obtained using stepwise regression with the full model forms in Equations (5.5) and (5.8), respectively. Table 7.9 shows that the corresponding component concentration models based on Equations (5.5) and (5.8) have similar $\mathrm{R}^{2}$ values, with a few exceptions. Those exceptions involve lower $\mathrm{R}^{2}$ values for the models based on Equation (5.8), which include the $\ln \left(C_{S S}\right)$ model for the 120-inch tank (which has a statistically significant LOF) and the $\ln \left(C_{\mathrm{ZrO} 2}\right)$ models for both the 43-inch and 120-inch tanks. 
Table 7.9. Model Terms, $R^{2}$ Values, and Model Lack-of-Fit Assessment for Stepwise Regression Fits of Natural Logarithm of Component Concentrations in Models (5.5) and (5.8), Separately for Each of the Two Scaled Tanks

\begin{tabular}{|c|c|c|c|c|c|}
\hline $\begin{array}{l}\text { Tank } \\
\text { Dia. } \\
\text { (in.) }\end{array}$ & $\ln ($ Component $)$ & $\begin{array}{c}\text { Model } \\
\text { Equation }\end{array}$ & Model Terms $^{(\mathbf{a})}$ & $\mathbf{R}^{2(b)}$ & $\begin{array}{l}\text { LOF Significant } \\
\text { with } 95 \% \\
\text { Confidence }\end{array}$ \\
\hline \multirow{8}{*}{43} & \multirow{2}{*}{$\ln$ (gibbsite) } & $(5.5)$ & 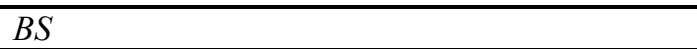 & 0.9940 & No \\
\hline & & $(5.8)$ & $\ln (U), B S, K V, \ln (U)^{*} B S, \ln (U)^{*} K V, K V^{2}$ & 0.9946 & No \\
\hline & \multirow{2}{*}{$\ln$ (stainless steel) } & $(5.5)$ & $\begin{array}{l}U, B S, K V, C V, B N, U^{2}, K V^{2}, B S^{*} K V, U^{*} K V^{2}, \\
U^{2} * K V^{2}, U^{*} B N\end{array}$ & 0.9318 & No \\
\hline & & $(5.8)$ & $\begin{array}{l}\ln (U), B S, K V, C V, B N,[\ln (U)]^{2}, K V^{2}, B S^{*} K V, \\
\ln (U)^{*} K V^{2},[\ln (U)]^{2 *} K V^{2}, \ln (U)^{*} B N\end{array}$ & 0.9350 & No \\
\hline & \multirow{2}{*}{$\ln ($ sand $)$} & $(5.5)$ & $\begin{array}{l}U, B S, K V, B N, U^{2}, U^{*} B S, U^{2} * B S, B S^{*} B N \\
K V^{*} B N\end{array}$ & 0.9784 & No \\
\hline & & $(5.8)$ & $\begin{array}{l}\ln (U), B S, K V, B N, \ln (U)^{*} B S, B S^{*} B N, K V^{*} B N, \\
{[\ln (U)]^{2},[\ln (U)]^{2} B S}\end{array}$ & 0.9785 & No \\
\hline & \multirow{2}{*}{$\ln \left(\mathrm{ZrO}_{2}\right)$} & $(5.5)$ & $\begin{array}{l}U, B S, K V, C V, B N, U^{2}, U^{3}, K V^{2}, U^{*} B S, U^{2 *} B S, \\
B S^{*} K V, U^{*} K V, U^{*} K V^{2}, U^{2 *} K V, B S^{*} B N\end{array}$ & 0.8151 & No \\
\hline & & $(5.8)$ & $\ln (U), B S, K V, C V, B S^{*} K V$ & 0.7652 & No \\
\hline \multirow{8}{*}{120} & \multirow{2}{*}{$\ln$ (gibbsite) } & $(5.5)$ & $B S, K V, C V, K V^{2}$ & 0.9916 & No \\
\hline & & $(5.8)$ & $B S, K V, C V, K V^{2}$ & 0.9916 & No \\
\hline & \multirow[t]{2}{*}{$\ln$ (stainless steel) } & $(5.5)$ & $\begin{array}{l}U, B S, K V, C V, B N, U^{2}, U^{3}, K V^{2}, U^{*} B S, U^{2 *} B S, \\
U^{*} K V, U^{*} K V^{2}, U^{2 *} K V^{2}, B S^{*} B N, K^{*} B N, \\
U^{*} B S^{*} B N\end{array}$ & 0.9323 & No \\
\hline & & $(5.8)$ & $\begin{array}{l}\ln (U), B S, K V, C V, B N, \ln (U)^{*} K V,[\ln (U)]^{2} \\
{[\ln (U)]^{2} * K V}\end{array}$ & 0.9074 & Yes \\
\hline & \multirow{2}{*}{$\ln ($ sand $)$} & $(5.5)$ & $U, B S, K V, C V, B N, B S^{*} B N, K V^{*} B N$ & 0.9551 & No \\
\hline & & $(5.8)$ & $\ln (U), B S, K V, C V, B N, B S^{*} B N, K V^{*} B N$ & 0.9552 & No \\
\hline & \multirow[t]{2}{*}{$\ln \left(\mathrm{ZrO}_{2}\right)$} & $(5.5)$ & $\begin{array}{l}U, B S, K V, C V, U^{2}, U^{3}, K V^{2}, U^{*} B S, U^{2} * B S, \\
B S^{*} K V, U^{*} K V^{2}\end{array}$ & 0.6906 & No \\
\hline & & $(5.8)$ & $B S, K V, C V, K V^{2}$ & 0.6469 & No \\
\hline \multicolumn{6}{|c|}{$\begin{array}{l}\text { (a) } U=\text { mixer-jet nozzle velocity, } B S=\text { base simulant, } K V=\text { kinematic viscosity, } C V=\text { capture velocity, and } \\
B N=\text { batch number ( } 0 \text { for pre-transfer, } 1-5 \text { for transfer batches). Finally, all models contained an intercept } \\
\text { term, which is not shown for simplicity. Coded values of some parameters were used in modeling (see } \\
\text { Section } 7 \text { ). } \\
\text { (b) } \mathrm{R}^{2} \text { is the fraction of variation in } \ln \text { (concentration) data accounted for by a model. }\end{array}$} \\
\hline
\end{tabular}

Per the preceding discussion based only on $\mathrm{R}^{2}$ values, the component concentration models in Section 7.1 (based on Equation (5.5)) perform as well or better than the models in Section 7.2 (based on Equation (5.8)) and do not have statistically significant LOFs. The models in Section 7.1 and 7.2 were additionally assessed by comparing their PvM plots: Figure 7.1 versus Figure 7.5, Figure 7.2 versus Figure 7.6, Figure 7.3 versus Figure 7.7, and Figure 7.4 versus Figure 7.8. The only noticeable differences in the PvM plots were for the models from Section 7.1 that had higher $\mathrm{R}^{2}$ values than the corresponding models in Section 7.2; those cases also had better-looking PvM plots.

\subsection{Sensitivity of Model-Predicted Component Concentrations to Parameters in the Models}

The PPPs (as discussed in Section 5.4.3) are presented in this section for the SS and sand concentration models of Sections 7.1 and 7.2. The models for SS and sand are of more interest, which is 
why this section focuses on PPPs only for those models. The PPPs corresponding to the Section 7.1 and 7.2 models for gibbsite and $\mathrm{ZrO}_{2}$ concentrations are presented in Appendix D.

The PPPs in this section and Appendix D have model-predicted values of component concentrations on the $y$-axis, which was accomplished by exponentiating the $\ln \left(C_{j}\right)$ model predictions for the $j^{\text {th }}$ component. The $x$-axis is the range of actual mixer-jet nozzle velocities tested in the 43 -inch tank. Then, profiles with different color lines for different $K V$ values and different line styles (dashed, dotted, and solid) for different $\mathrm{CV}$ values are plotted for up to seven combinations of $K V$ and $C V$. If the model associated with a PPP is not a function of $C V$, then profiles are shown for three values of $K V$. Finally, if a model is not a function of velocity, the profiles are shown as horizontal lines.

The PPPs provide additional information that is useful in choosing between the two alternative models (Section 7.1 or 7.2) for a given component concentration. The PPPs also provide a graphical basis for assessing how sensitive the model predictions of component concentrations are to changes in the parameters included in the models. Finally, note that although PPPs are interpolating within the ranges of the test values of $B S, U, K V$, and $C V$, it is possible that predictions of component concentrations may fall outside the concentration ranges observed in testing. This can occur because the 22 distinct test combinations provided a very fractionated exploration of the four-dimensional parameter space of ( $B S, U$, $K V$, and $C V$ ), as discussed in Section 2.2. Model predictions for subregions of this space not experimentally explored could be extrapolations in the sense of the predicted concentrations being smaller or larger than values observed during testing. This could be acceptable or an indication of model predictions "going too far" and being questionable for some subregions of the parameter space.

The sensitivity of a component concentration to the parameters was assessed by comparing the average difference in concentration values seen over the range of values explored for each parameter keeping all other parameter values constant. For $B S$ the comparison was between the two plots (for $B S=$ $\mathrm{T}$ and $B S=\mathrm{H}$ ), whereas for the other parameters comparisons were made within a plot. The magnitudes of these average differences were used to rank the sensitivities of a component concentration to the parameters, with the largest average differences being associated with the parameter that had the largest sensitivity. These comparisons were done over the range of parameter values given in the PPP figures, which correspond to the target $S V$ and $C V$ experimental design conditions. $K V$ was calculated as $S V /(1.259+0.0076 S V)$ because the equation Density $=1.259+0.0076 S V$ was the basis used by Lee et al. (2012a) to determine the target densities used for testing. The target densities and resulting target $K V$ values are given in Table 7.10.

Table 7.10. Target $S V$, Density, and Resulting $K V$ Values Used in the Experimental Design and Prediction Profile Plots

\begin{tabular}{ccc}
\hline$S V(\mathrm{cP})$ & Density $=1.259+0.0076 S V(\mathrm{~g} / \mathrm{ml})$ & $K V(\mathrm{cSt})$ \\
\hline 3.3 & 1.284 & 2.57 \\
8.0 & 1.320 & 6.06 \\
14.6 & 1.370 & 10.66 \\
\hline
\end{tabular}




\subsubsection{Prediction Profile Plots for the Stainless Steel and Sand Concentration Models in Section 7.1}

Figure 7.9 and Figure 7.10 show the PPPs for the Table 7.2 models of SS concentrations in the 43inch and 120-inch tanks with $B S=$ Typical and High, respectively. These figures show that the sensitivity of SS concentration $\left(C_{S S}\right)$ to the parameters, from largest to smallest sensitivity, is $B S, U, K V$, and $C V$ for both the 43 -inch and 120-inch tanks. The following additional observations are made from Figures 7.9 and 7.10 .

- $C_{S S}$ is most sensitive to the $B S$ because of the different mass fractions of SS in the two base simulants.

- $C_{S S}$ is next most sensitive to $U$ after $B S$. Despite the differences in magnitudes of $C_{S S}$ between the two base simulants, the shapes of the $C_{S S}$ vs. $U$ profiles for the two simulants are roughly similar (with some differences) for a given size tank. The main difference is that for larger mixer-jet pump velocities $(U>38 \mathrm{ft} / \mathrm{sec})$ and $K V=6.1 \mathrm{cSt}$, the predicted $C_{S S}$ is larger than any concentration observed during testing.

- For the 43-inch tank with $B S=$ Typical, $C_{S S}$ tends to increase with $U$, although for $K V=2.6 \mathrm{cSt}$, $C_{S S}$ is predicted to level off at $\sim 31 \mathrm{ft} / \mathrm{sec}$ and decrease some from there. The tendency of $C_{S S}$ to increase with $U$ decreases with increasing $K V$.

- $\quad$ For the 43-inch tank with $B S=$ High, $C_{S S}$ tends to increase with $U$, although for $K V=2.6$ and 10.7 $\mathrm{cSt}, C_{S S}$ is predicted to level off and decrease some. That the leveling off in the $C_{S S}$ vs. $U$ profiles does not occur for $K V=6.1 \mathrm{cSt}$ is likely a result of the interaction terms involving $K V^{2}$ in the model of Table 7.2.

- $\quad$ For the 120-inch tank with $B S=$ Typical, the $C_{S S}$ vs. $U$ profiles are similar for $K V=2.6$ and 10.7 cSt, with an initial increase in $C_{S S}$ as $U$ increases, but then leveling off and decreasing some as $U$ increases further. These same profiles for the 120 -inch tank with $B S=$ High show a more consistent but larger increase in $C_{S S}$ with increasing $U$.

- For the 43-inch tank with both base simulants, $C_{S S}$ increases as $C V$ increases. For the 120 -inch tank, the increase in $C_{S S}$ with increasing $C V$ is smaller than for the 43-inch tank. For the 120-inch tank, the increase in $C_{S S}$ with increasing $C V$ is larger for $B S=$ High than for $B S=$ Typical. 

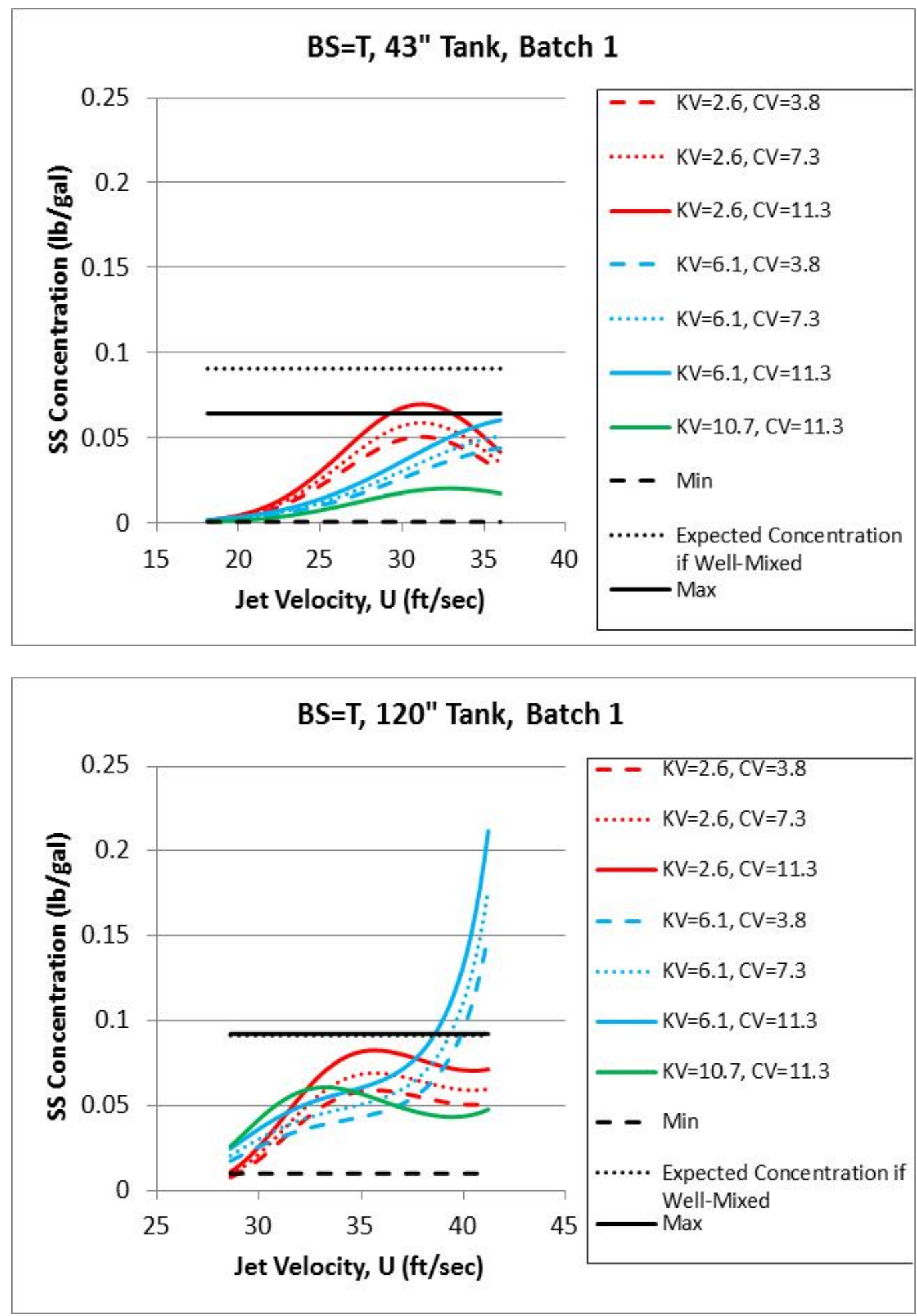

Figure 7.9. Prediction Profile Plot of Stainless Steel Concentrations versus Velocity Using the Models from Table 7.2 for Batch 1 from the 43-Inch and 120-Inch Tanks with Typical Base Simulant and Various Combinations of Kinematic Viscosity and Capture Velocity (For Information Only) 

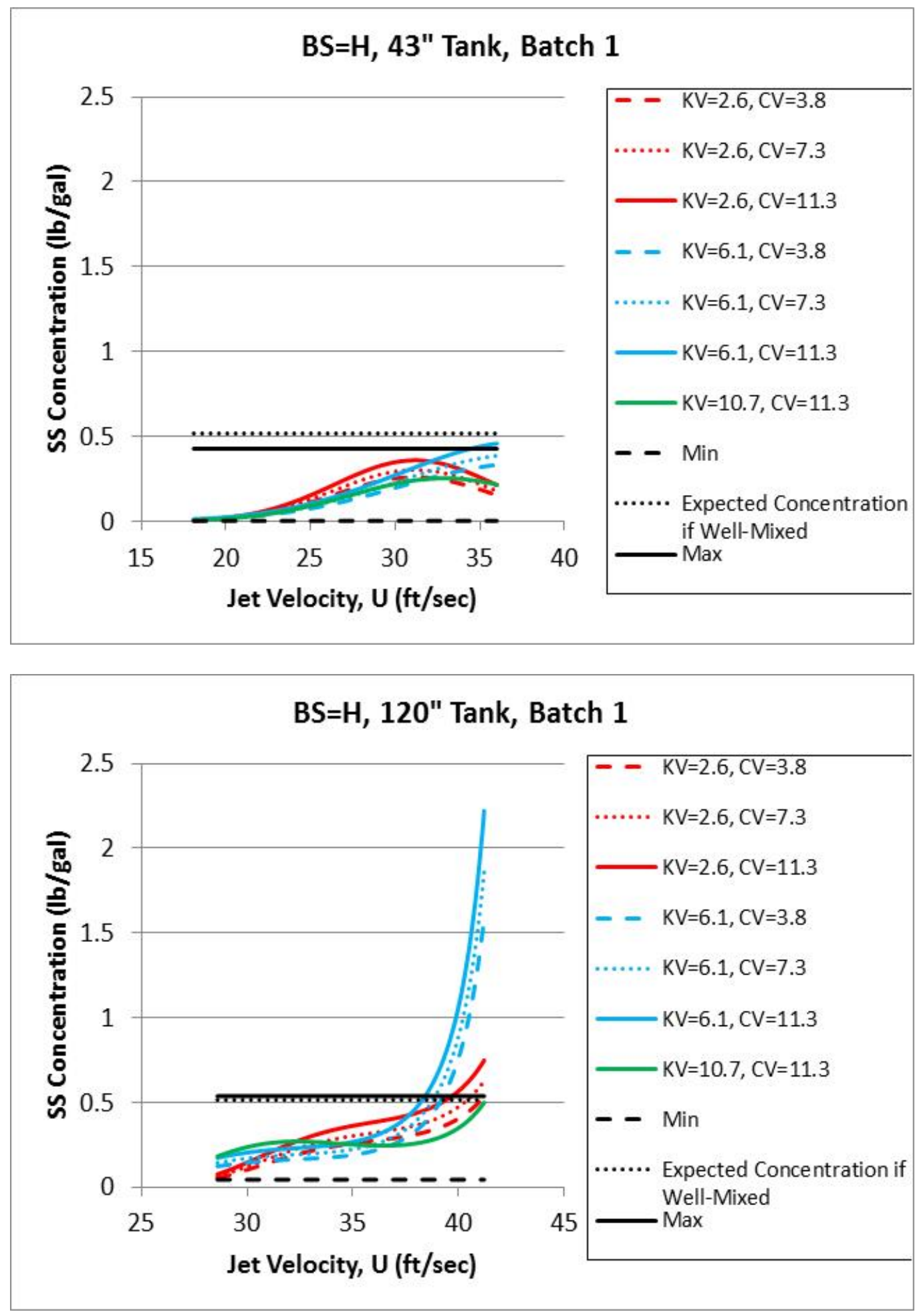

Figure 7.10. Prediction Profile Plot of Stainless Steel Concentrations versus Velocity Using the Models from Table 7.2 for Batch 1 from the 43-Inch and 120-Inch Tanks with High Base Simulant and Various Combinations of Kinematic Viscosity and Capture Velocity (For Information Only) 
Figure 7.11 and Figure 7.12 are analogous to Figures 7.9 and 7.10, except they are PPPs based on the Table 7.3 models for concentrations of sand. These figures show that the sensitivity of sand concentration $\left(C_{\text {sand }}\right)$ to the parameters, from largest to smallest sensitivity, depends on the tank size. For the 43-inch tank, the sensitivity order is $B S, U$, and $K V$ ( $C V$ is not included in the $C_{\text {sand }}$ model for the 43 -inch tank). For the 120-inch tank, the sensitivity order is $B S, C V, U$, and $K V$. The following additional observations are made from Figures 7.11 and 7.12.

- $C_{\text {sand }}$ is most sensitive to the $B S$ because of the different mass fractions of sand in the two base simulants.

- For the 43-inch tank, $C_{\text {sand }}$ is next most sensitive to $U$. $C_{\text {sand }}$ is predicted to increase with increasing $U$ for both $B S=$ Typical and $B S=$ High, but for $B S=$ Typical the profiles level off around $32 \mathrm{ft} / \mathrm{sec}$ and decrease a little with increases in $U$ beyond that value.

- For the 43-inch tank with both base simulants, $C_{\text {sand }}$ is predicted to have little sensitivity to $K V$. $C_{\text {sand }}$ may increase a minor amount with increasing $K V$.

- For the 120-inch tank, $C_{\text {sand }}$ is predicted to increase linearly with $U$, although the rate of increase (i.e., the slope) is much higher for $B S=$ High than $B S=$ Typical (as might be expected because there is more sand in the High base simulant).

- For the 120-inch tank, $C_{\text {sand }}$ is second most sensitive to $C V$ (more sensitive than to $U$ ), with $C_{\text {sand }}$ increasing as $C V$ increases. The increase in $C_{\text {sand }}$ with $\mathrm{CV}$ is much stronger (higher slope) for $B S$ $=$ High than for $B S=$ Typical (as might be expected because of the higher amount of sand in the High base simulant).

- For the 120-inch tank for both $B S=$ Typical and $B S=$ High, $C_{\text {sand }}$ is predicted to decrease as $K V$ increases. The decreases are larger for $B S=$ High compared to $B S=$ Typical, presumably because of the higher amount of sand in the High base simulant. 

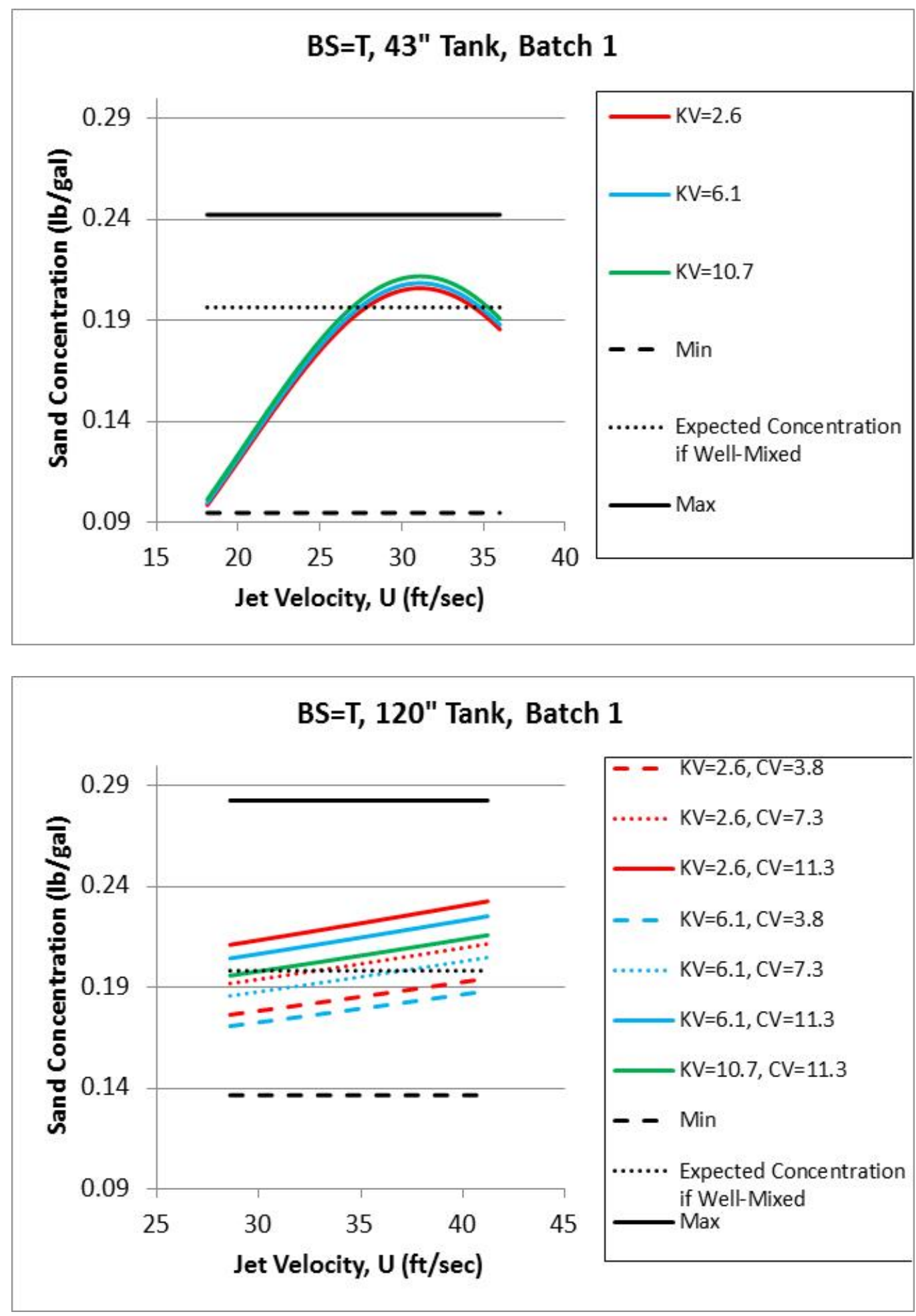

Figure 7.11. Prediction Profile Plot of Sand Concentrations versus Velocity Using the Models from Table 7.3 for Batch 1 from the 43-Inch and 120-Inch Tanks with Typical Base Simulant and Various Combinations of Kinematic Viscosity and Capture Velocity (For Information Only) 

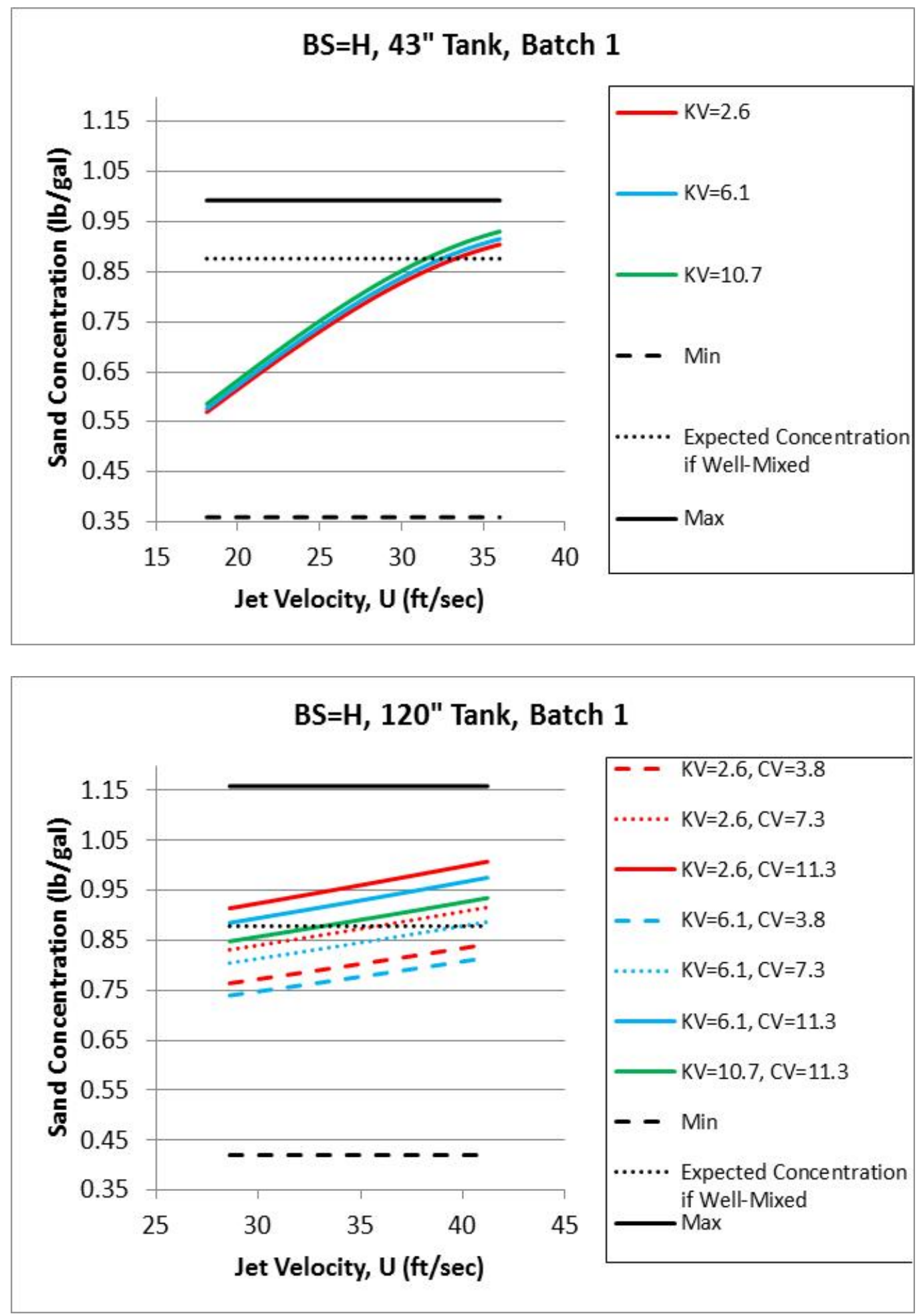

Figure 7.12. Prediction Profile Plot of Sand Concentrations versus Velocity Using the Models from Table 7.3 for Batch 1 from the 43-Inch and 120-Inch Tanks with High Base Simulant and Various Combinations of Kinematic Viscosity and Capture Velocity (For Information Only) 


\subsubsection{Prediction Profile Plots for the Stainless Steel and Sand Concentration Models in Section 7.2}

Figure 7.13 and Figure 7.14 show the PPPs for the Table 7.6 models of SS concentrations in the 43inch and 120-inch tanks with $B S=$ Typical and High, respectively. These figures show that the sensitivity of SS concentration $\left(C_{S S}\right)$ to the parameters, from largest to smallest sensitivity, is $U, B S, K V$, and $C V$ for the 43-inch tank and $B S, U, K V$, and $C V$ for the 120-inch tank. The following additional observations are made from Figures 7.13 and 7.14.

- $C_{S S}$ is most sensitive to the $B S$ because of the different mass fractions of SS in the two base simulants.

- $C_{S S}$ is next most sensitive to $U$. Despite the differences in magnitudes of $C_{S S}$ between the two base simulants, the shapes of the $C_{S S}$ vs. $U$ profiles for the two simulants are (i) very similar for the 120-inch tank and (ii) similar for the 43-inch tank, with the profile differences less pronounced for $B S=\mathrm{H}$ than $B S=$ Typical.

- For the 43-inch tank with $B S=$ Typical, $C_{S S}$ tends to increase with $U$, although for $K V=2.6 \mathrm{cSt}$, $C_{S S}$ is predicted to level off at $\sim 31 \mathrm{ft} / \mathrm{sec}$ and decrease some from there. The tendency of $C_{S S}$ to increase with $U$ decreases with increasing $K V$.

- $\quad$ For the 43-inch tank with $B S=$ High, $C_{S S}$ tends to increase with $U$, although for $K V=2.6 \mathrm{cSt}, C_{S S}$ is predicted to level off and decrease a little. That the leveling off in the $C_{S S}$ vs. $U$ profiles does not occur for $K V=6.1$ and $10.7 \mathrm{cSt}$ is likely a result of the interaction terms involving $K V$ in the model of Table 7.6.

- For the 43-inch tank with $B S=$ High, $C_{S S}$ is predicted to exceed the maximum observed SS concentration for $K V=6.1 \mathrm{cSt}$ and $C V=11.3 \mathrm{ft} / \mathrm{sec}$ for $U_{43}>34 \mathrm{ft} / \mathrm{sec}$. For $B S=$ Typical a similar, but less extreme trend is seen for $U_{43}>35 \mathrm{ft} / \mathrm{sec}$.

- For the 120-inch tank with $B S=$ Typical and High, the $C_{S S}$ vs. $U$ profiles for all $K V$ values are similar up to $\sim U_{120}=37 \mathrm{ft} / \mathrm{sec}$. At that point the profiles for $K V=26$ and $6.1 \mathrm{cSt}$ level off or start to level off the profile for $K V=10.7$ continues increasing. For $B S=$ Typical, the profile for $K V=$ 10.7 increases past the maximum observed SS concentration for $U_{120}>40.6 \mathrm{ft} / \mathrm{sec}$. 

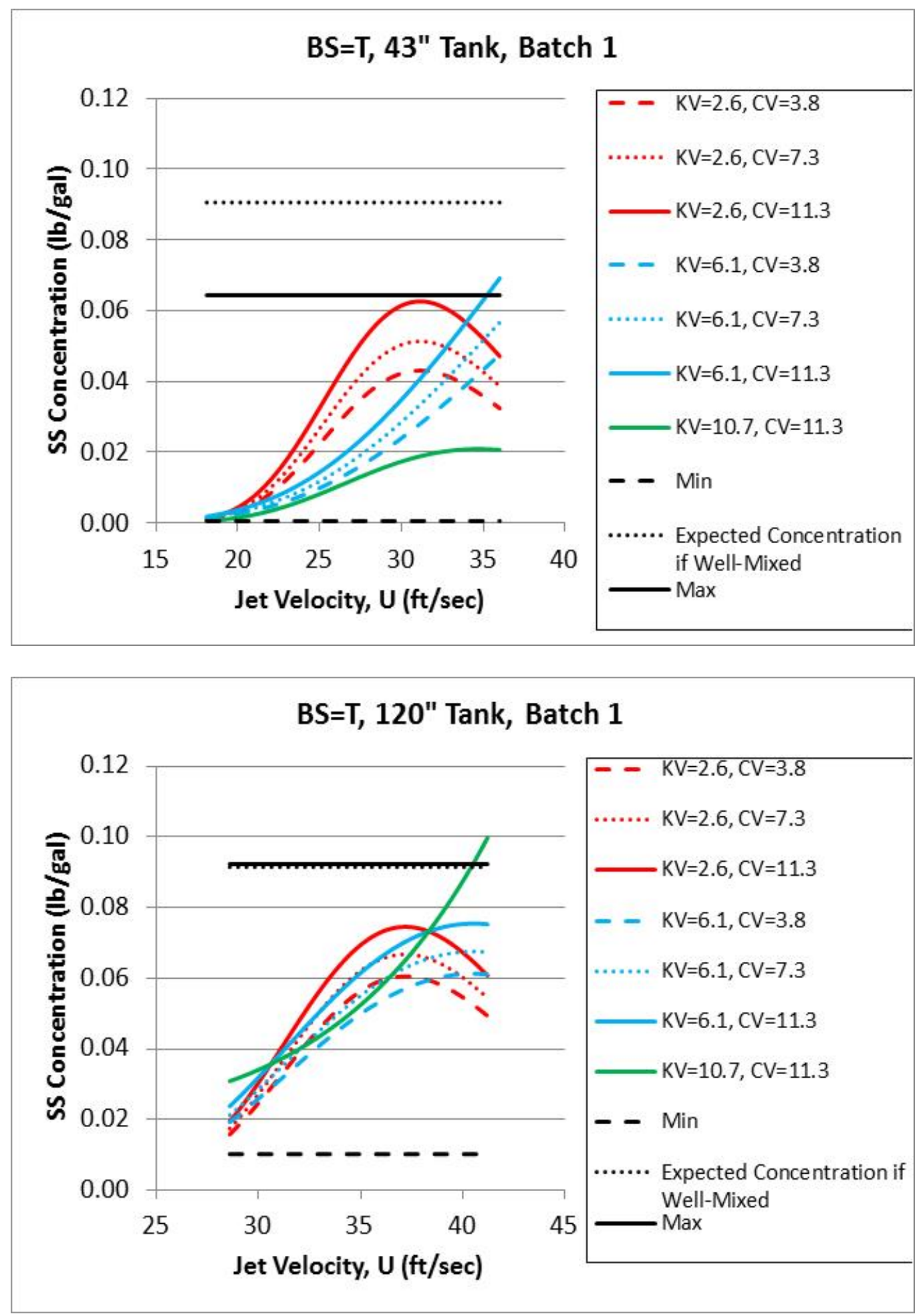

Figure 7.13. Prediction Profile Plot of Stainless Steel Concentrations versus Velocity Using the Model from Table 7.2 for Batch 1 from the 43-Inch and 120-Inch Tanks with Typical Base Simulant and Various Combinations of Kinematic Viscosity and Capture Velocity (For Information Only) 

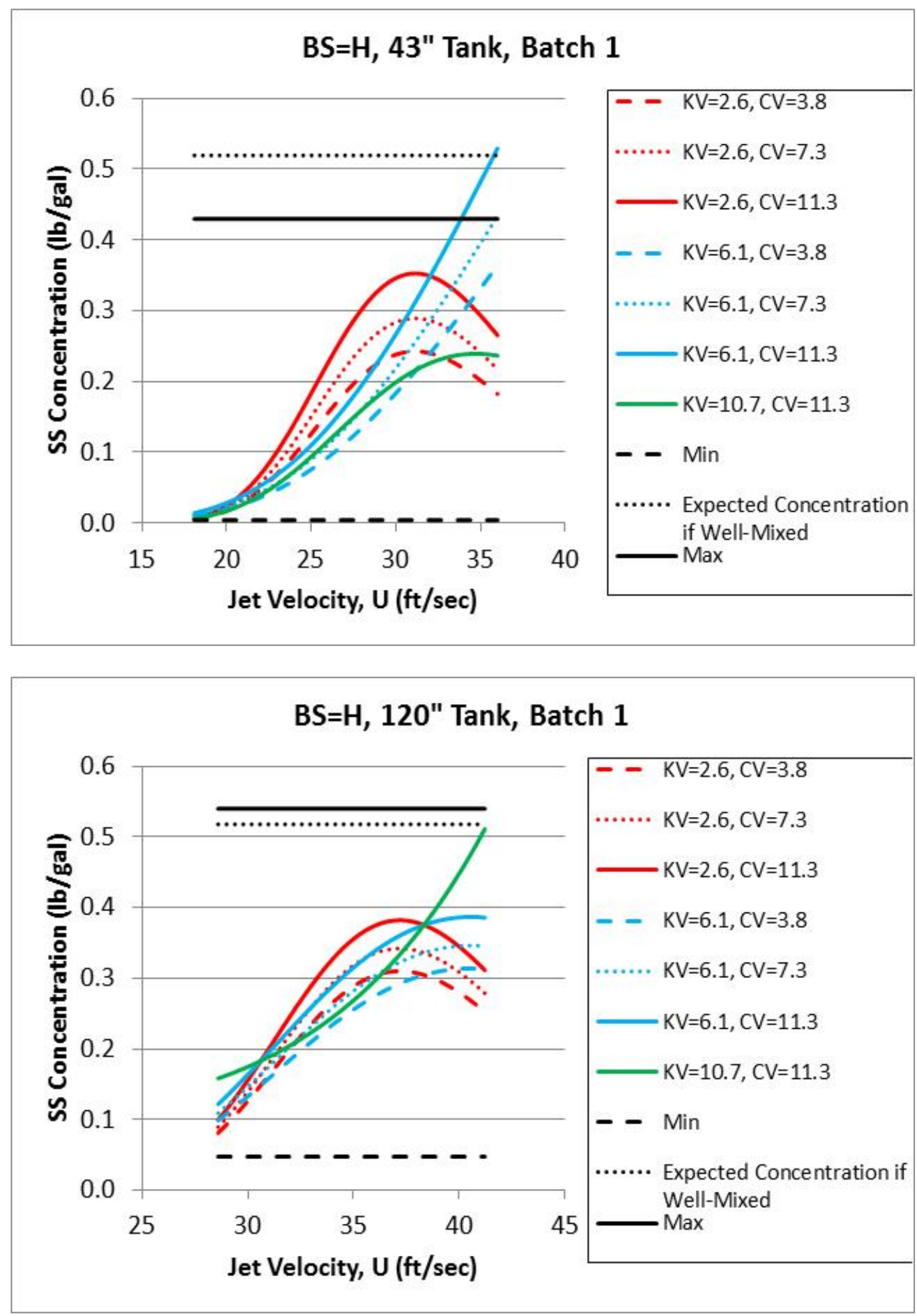

Figure 7.14. Prediction Profile Plot of Stainless Steel Concentrations versus Velocity Using the Model from Table 7.2 for Batch 1 from the 43-Inch and 120-Inch Tanks with High Base Simulant and Various Combinations of Kinematic Viscosity and Capture Velocity (For Information Only) 
- For the 43-inch and 120-inch tanks with both base simulants, $C_{S S}$ increases as $C V$ increases. The amount of increase in $C_{S S}$ with increasing $C V$ is similar for both tanks with $B S=$ Typical. With $B S=$ High, the amount of increase in $C_{S S}$ with increasing $C V$ is a little less for the 43-inch tank than for the 120-inch tank.

Figure 7.15 and Figure 7.16 are analogous to Figures 7.13 and 7.14, except they are PPPs based on the Table 7.7 models for concentrations of sand. These figures show that the sensitivity of sand concentration $\left(C_{\text {sand }}\right)$ to the parameters, from largest to smallest sensitivity, depends on the tank size. For the 43-inch tank, the sensitivity order is $B S, U$, and $K V\left(C V\right.$ is not included in the $C_{\text {sand }}$ model for the 43inch tank). For the 120-inch tank, the sensitivity order is $B S, C V, U$, and $K V$. The following additional observations are made from Figures 7.15 and 7.16.

- $C_{\text {sand }}$ is most sensitive to the $B S$ because of the different mass fractions of sand in the two base simulants.

- For the 43-inch tank, $C_{\text {sand }}$ is next most sensitive to $U$. $C_{\text {sand }}$ is predicted to increase with increasing $U$ for both $B S=$ Typical and $B S=$ High. However, for $B S=$ Typical the profiles level off around $32 \mathrm{ft} / \mathrm{sec}$ and decrease a little with increases in $U$ beyond that value.

- For the 43-inch tank with both base simulants, $C_{\text {sand }}$ is predicted to have little sensitivity to $K V$. $C_{\text {sand }}$ may increase a minor amount with increasing $K V$.

- For the 120-inch tank, $C_{\text {sand }}$ is predicted to increase linearly with $U$, although the rate of increase (i.e., the slope) is much higher for $B S=$ High than $B S=$ Typical (as might be expected because there is more sand in the High base simulant).

- For the 120-inch tank, $C_{\text {sand }}$ is second most sensitive to $C V$ (more sensitive than to $U$ ), with $C_{\text {sand }}$ increasing as $C V$ increases. The increase in $C_{\text {sand }}$ with $C V$ is much stronger (higher slope) for $B S$ $=$ High than for $B S=$ Typical (as might be expected because of the higher amount of sand in the High base simulant).

- For the 120-inch tank for both $B S=$ Typical and $B S=$ High, $C_{\text {sand }}$ is predicted to decrease as $K V$ increases. The decreases are larger for $B S=$ High compared to $B S=$ Typical, presumably because of the higher amount of sand in the High base simulant. 

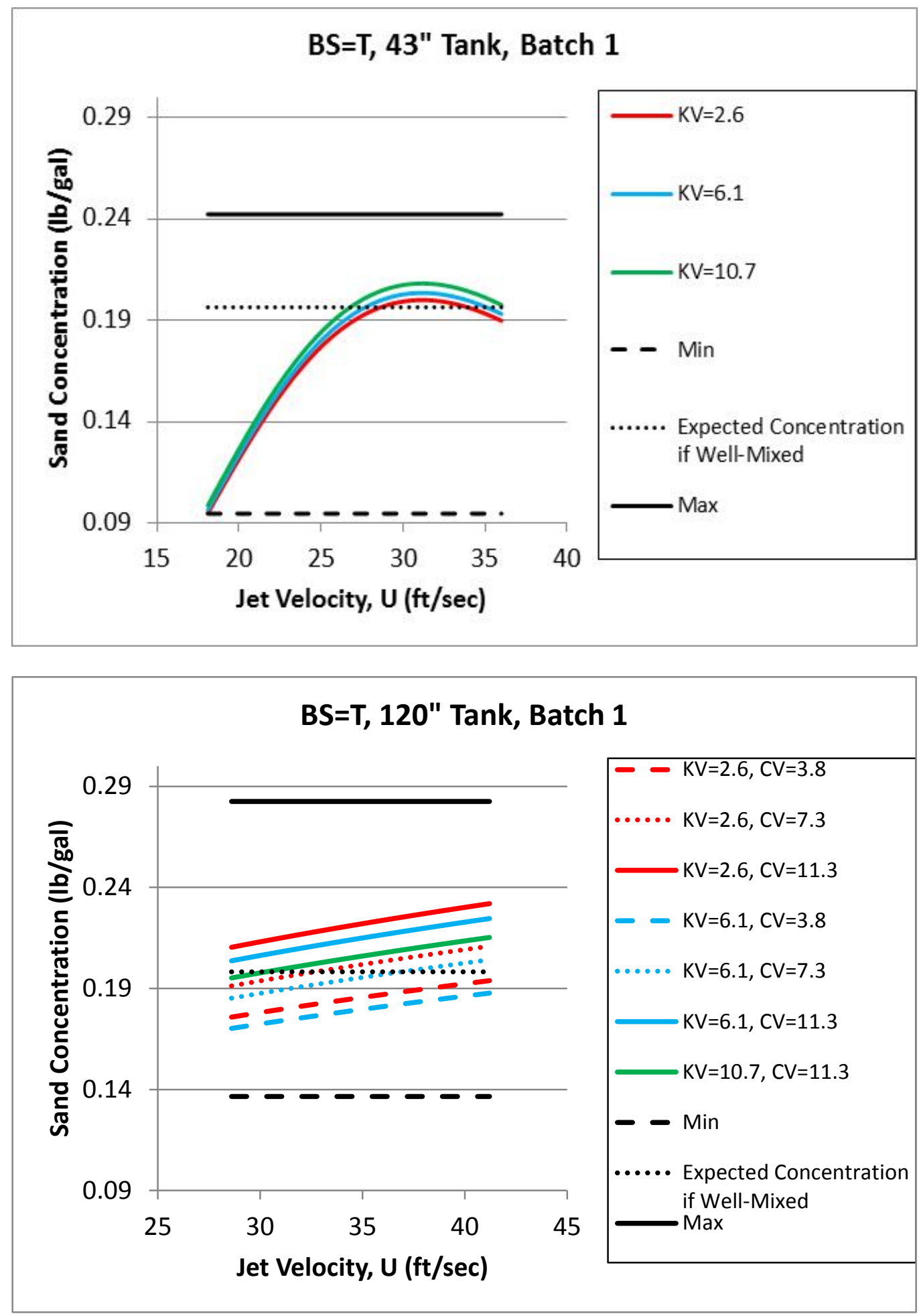

Figure 7.15. Prediction Profile Plot of Sand Concentrations versus Velocity Using the Model from Table 7.2 for Batch 1 from the 43-Inch and 120-Inch Tanks with Typical Base Simulant and Various Combinations of Kinematic Viscosity and Capture Velocity (For Information Only) 

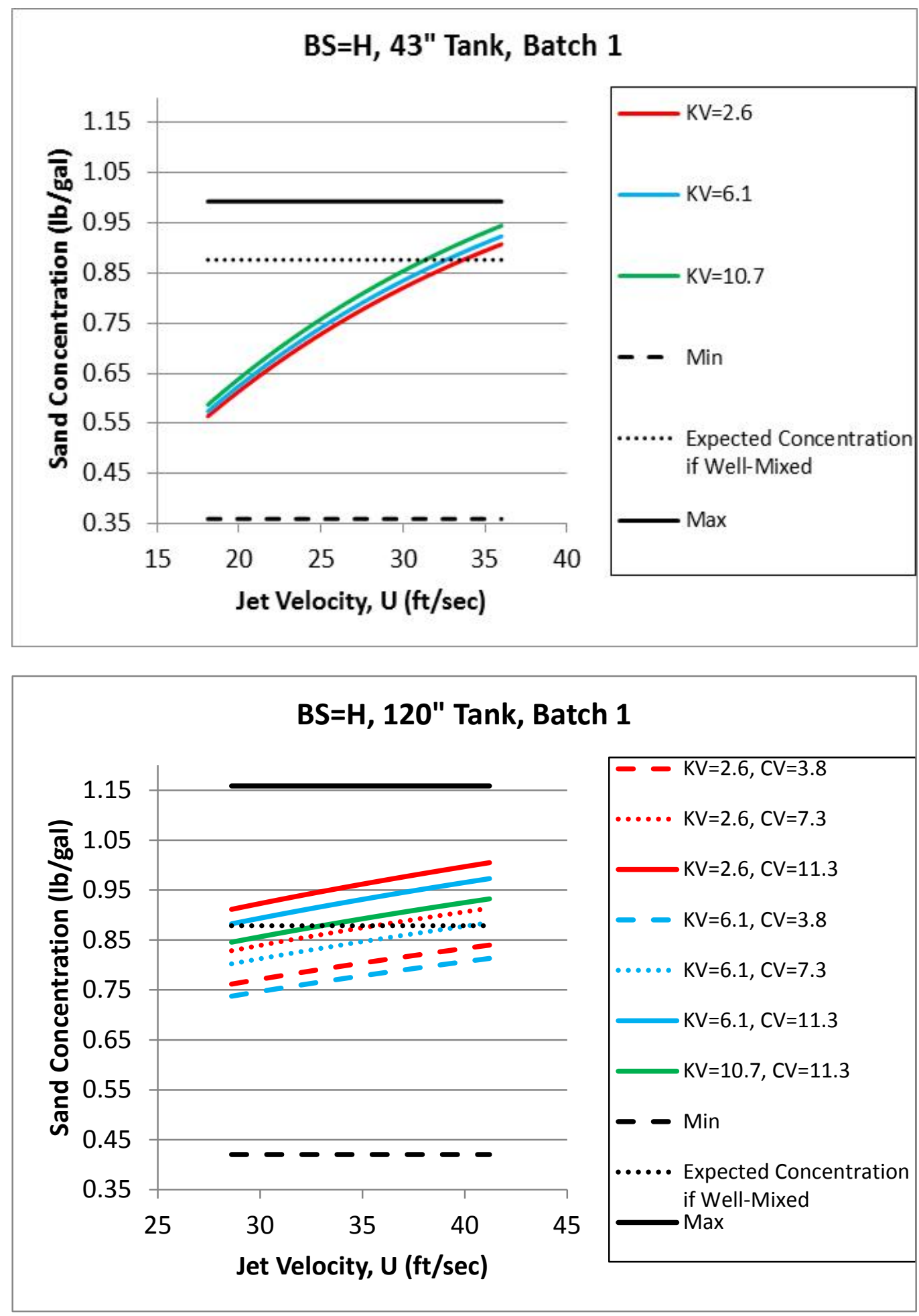

Figure 7.16. Prediction Profile Plot of Sand Concentrations versus Velocity Using the Model from Table 7.2 for Batch 1 from the 43-Inch and 120-Inch Tanks with High Base Simulant and Various Combinations of Kinematic Viscosity and Capture Velocity (For Information Only) 


\subsubsection{Comparison of Prediction Profile Plots for Stainless Steel and Sand Produced Using the Models from Section 7.1 and Section 7.2}

PPPs for concentrations of SS and sand using the model forms of Section 7.1 and 7.2) are compared to assess the differences in the model forms considered. The figures of interest to compare are

- $\quad$ Figure 7.9 and Figure 7.13 (SS with $B S=$ Typical)

- Figure 7.10 and Figure 7.14 (SS with $B S=$ High)

- $\quad$ Figure 7.11 and Figure 7.15 (Sand with $B S=$ Typical)

- Figure 7.12 and Figure 7.16 (Sand with $B S=$ High).

To aid in making these comparisons, the above pairs of figures are displayed in Figure 7.17 through Figure 7.20, respectively.

The pairs of PPPs for sand concentrations in Figure 7.19 and Figure 7.20 are very similar, such that there appears to be no practical difference between using models from Section 7.1 versus models from Section 7.2 to predict sand concentrations. However, there are noticeable differences in the pairs of PPPs for SS concentrations in Figure 7.17 and Figure 7.18, with some of the differences being substantial. The differences in Figure 7.17 and Figure 7.18 are discussed in what follows.

In Figure 7.17, the predicted $C_{S S}$ values in the PPPs for the 43 -inch tanks are very similar. The same is true of the PPPs for the 43-inch tank in Figure 7.18. However, there are noticeable differences between the PPPs in Figures 7.17 and 7.18 for 120-inch tanks on the left (models in Table 7.2) and right (models in Table 7.6).

1. The biggest difference in $C_{S S}$ profiles is for PPPs of 120-inch tanks in Figure 7.17 and Figure 7.18 , where profiles for $K V=6.1 \mathrm{cSt}$ on the left (Table 7.2 models) increase rapidly for $U_{120}>37$ $\mathrm{ft} / \mathrm{sec}$. The predicted $C_{S S}$ values at the largest $U_{120}$ are more than twice the largest values observed during testing for the two base simulants. The PPPs produced with the Table 7.6 models do not show this same "overprediction" problem. Even though the PPPs are based on interpolating the models inside the parameter space that was tested, there was only one test performed with $K V$ close to $6.1 \mathrm{cSt}$ and $U_{120}>37 \mathrm{ft} / \mathrm{sec}$. It was test number 15 in the 120 -inch tank and had a $K V$ of $5.82 \mathrm{cSt}$ and an average mixer-jet pump velocity of $38.55 \mathrm{ft} / \mathrm{sec}$ over the 5 batches.

2. In Figure 7.18, the $K V=2.6 \mathrm{cSt}$ profiles for the 120 -inch tank on the left (Table 7.2 model) also begin to rise rapidly at the largest $U_{120}$ value, a behavior that does not occur in the PPP for the 120 -inch tank on the right (Table 7.6 model).

3. In Figure 7.17, for the 120-inch tank, the profile for $K V=10.7 \mathrm{cSt}$ and $C V=11.3 \mathrm{ft} / \mathrm{sec}$ is roughly horizontal for the PPP on the left (Table 7.2 model), whereas the profile steadily increases with $U_{120}$ for the PPP on the right (Table 7.6 model).

Based on the above observations, the models for SS concentrations in Table 7.6 are preferred to those in Table 7.2. Because there is little difference between the PPPs based on the models in Table 7.3 and Table 7.7 for sand concentrations, it would be reasonable to use the same type of model as chosen for SS concentrations, namely the Table 7.6 models. 

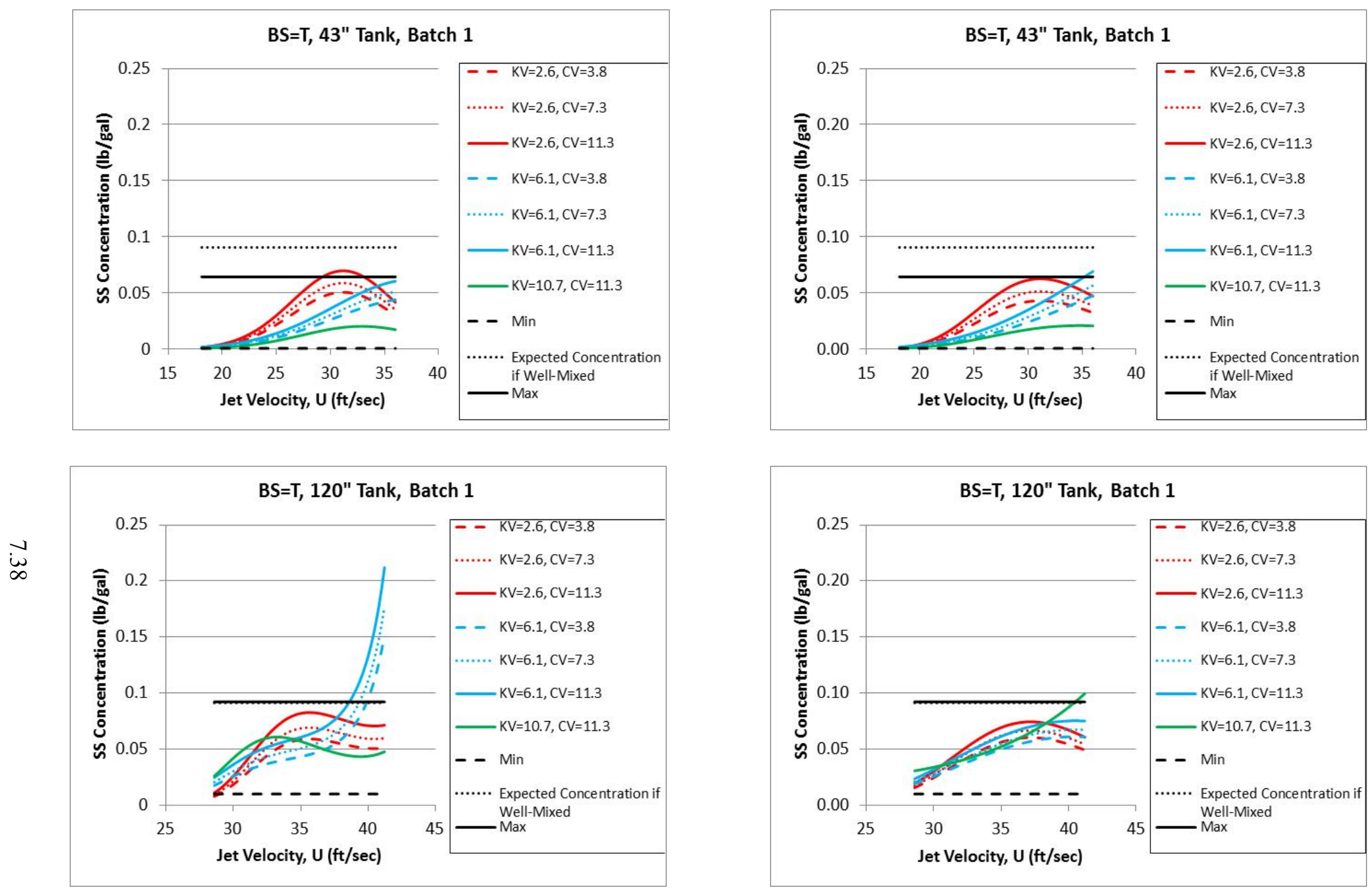

Figure 7.17. Comparison of Stainless Steel Prediction Profile Plots for Typical Base Simulant Produced Using the Models in Tables 7.2 and 7.6. The plots on the left are from Figure 7.9 (Table 7.2) and the plots on the right are from Figure 7.13 (Table 7.6). (For Information Only) 

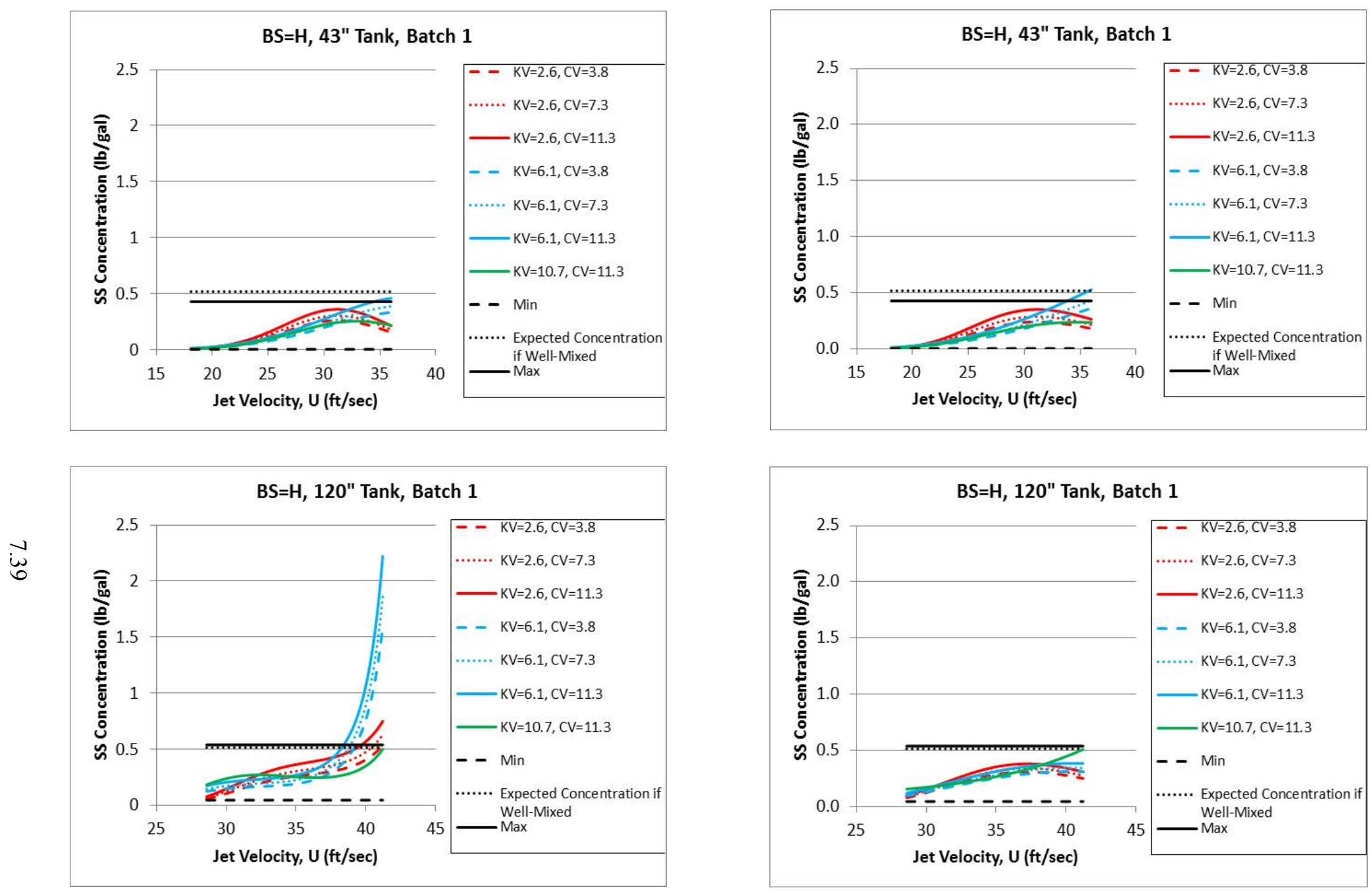

Figure 7.18. Comparison of Stainless Steel Prediction Profile Plots for Typical Base Simulant Produced Using the Models in Tables 7.2 and 7.6. The plots on the left are from Figure 7.10 (Table 7.2) and the plots on the right are from Figure 7.14 (Table 7.6). (For Information Only) 

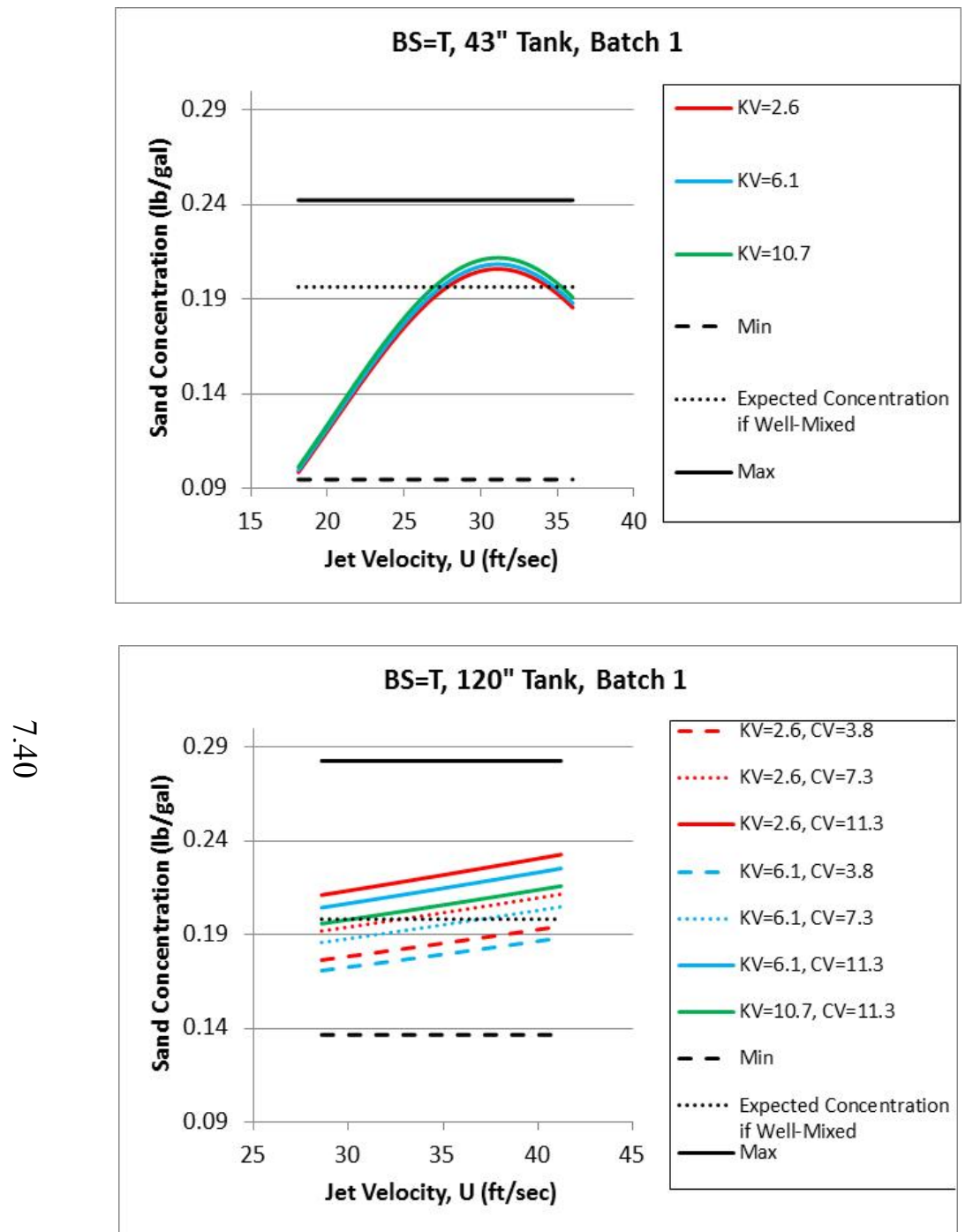
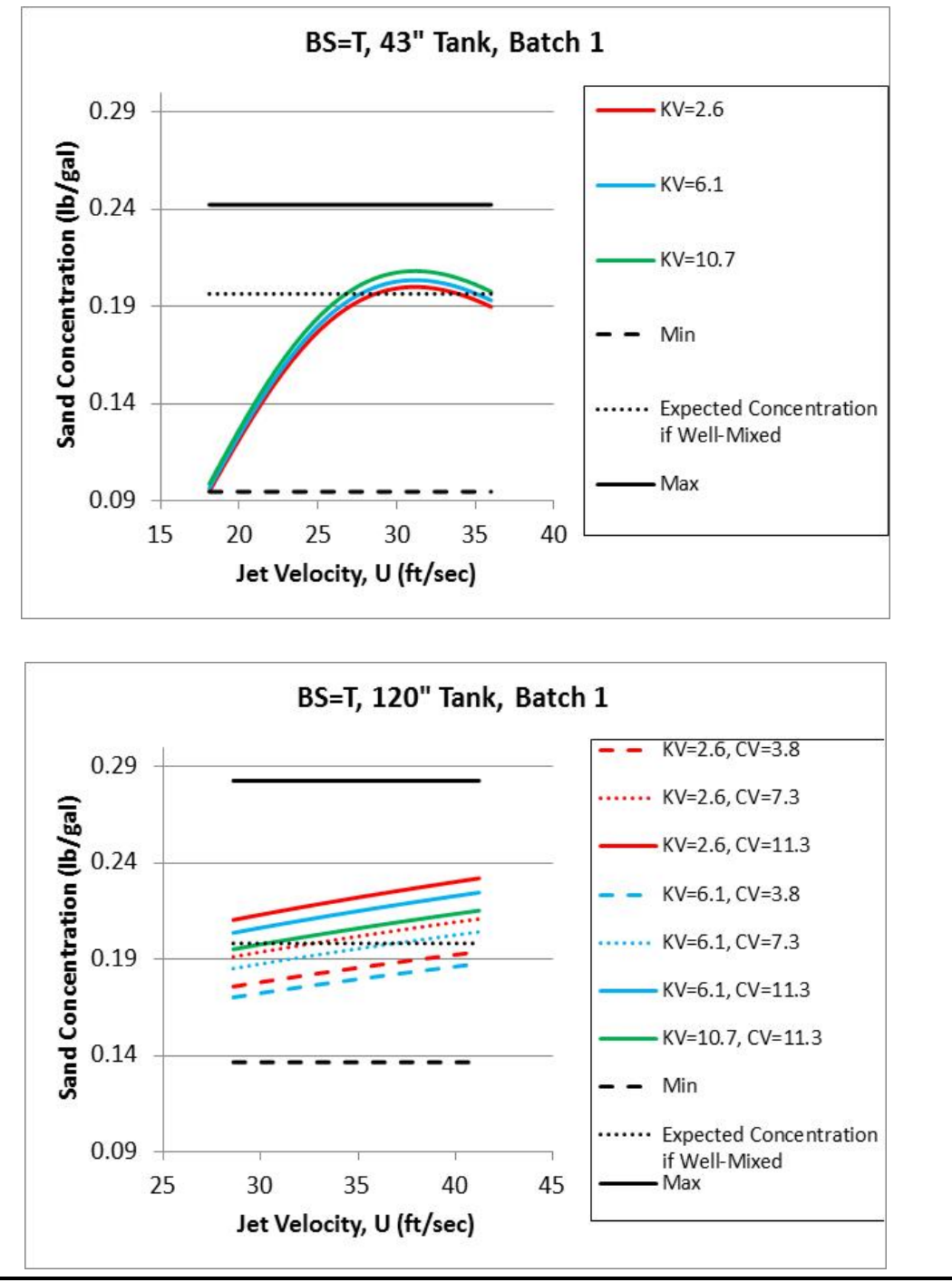

Figure 7.19. Comparison of Sand Prediction Profile Plots for Typical Base Simulant Produced Using the Models in Tables 7.3 and 7.7. The plots on the left are from Figure 7.11 (Table 7.3) and the plots on the right are from Figure 7.15 (Table 7.7). (For Information Only) 

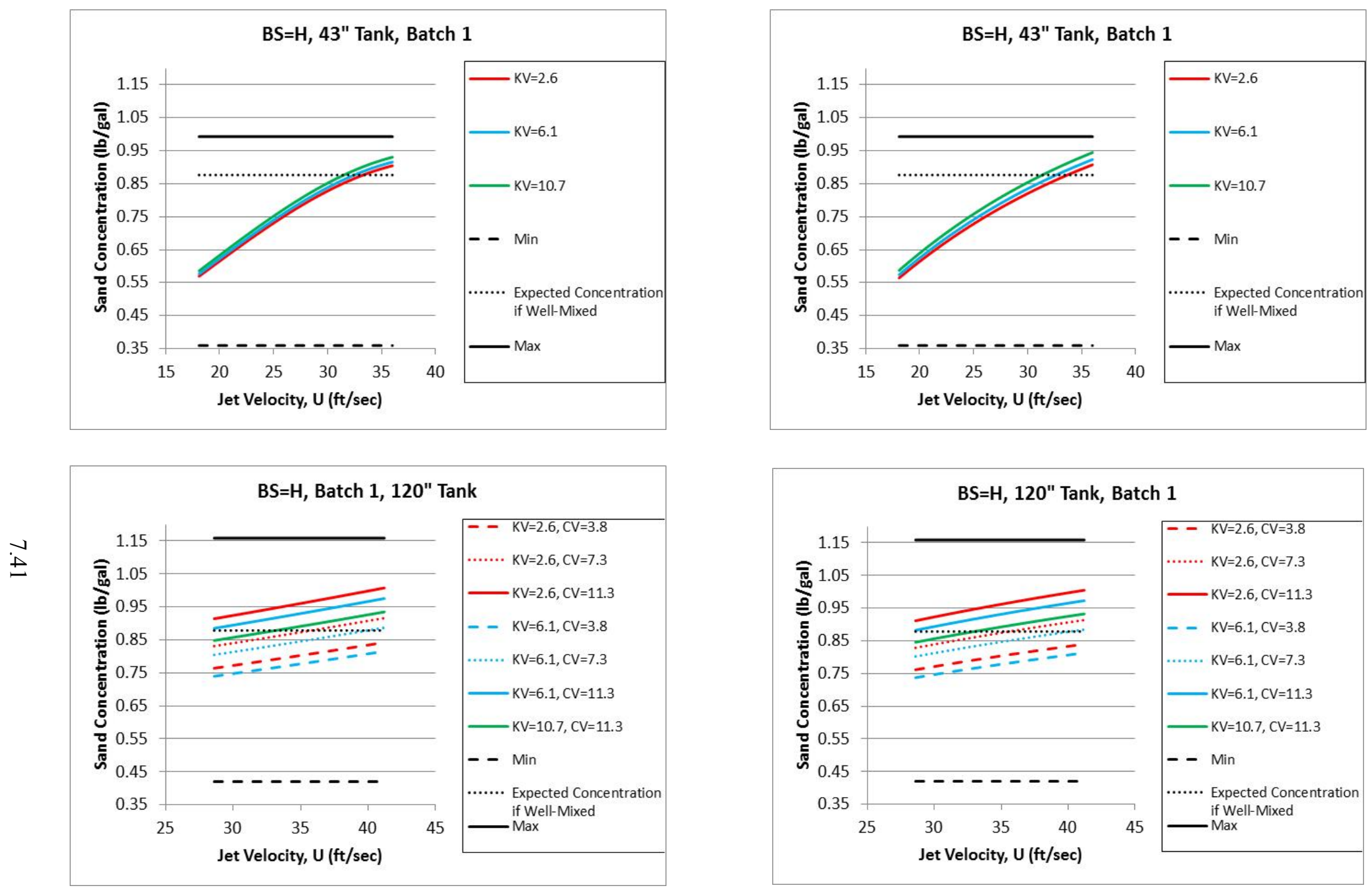

Figure 7.20. Comparison of Sand Prediction Profile Plots for Typical Base Simulant Produced Using the Models in Tables 7.3 and 7.7. The plots on the left are from Figure 7.12 (Table 7.3) and the plots on the right are from Figure 7.16 (Table 7.7). (For Information Only) 


\subsection{Scaling Exponents as Functions of Test Parameters Calculated Using the Equivalent Performance Approach}

This section utilizes the models for concentrations of gibbsite, $\mathrm{SS}$, sand, and $\mathrm{ZrO}_{2}$ in the 43-inch tank and 120-inch tank (as presented in Sections 7.1 and 7.2) to calculate values of the scaling exponent (a) according to the EP approach discussed in Section 6.2. According to Step 1 of the EP procedure discussed in Section 6.2, ranges of actual values of $U, K V$, and $C V$ from testing were used to construct the grid of points for which scaling exponents were calculated. In forming the grid of points, the constraint on $(\mathrm{KV}, \mathrm{CV})$ combinations specified by Equation (6.3) was used. The concentration models presented in Sections 7.1 and 7.2 are, in general, complex functions of parameters varied during testing. Therefore, it is possible that more than one value of " $a$ " can be found when matching concentrations between the 43inch and the 120-inch tanks for a given component and grid point per the EP approach in Section 6.2. To deal with this possibility, the value of " $a$ " used in Section 8.1 and 8.2, if more than one was found, is the one where the velocities for the 43-inch and the 120-inch tanks are closest, while maintaining calculated component concentration in the 120 -inch tanks within $20 \%$ of the target value in the 43 -inch tank.

The dependence of the " $a$ " values on the parameters $U, B S, K V, C V$, and $B N$ is discussed. Sections 8.1 and 8.2 present the scaling exponent results using the modeling results from Sections 7.1 and 7.2, respectively. However, note that the EP approach discussed in Section 6.2 cannot be applied if the model of a given type (Section 7.1 or Section 7.2) for either one (or both) of the 43-inch tank or 120-inch tank does not depend on $U$.

The subsections within each of Section 8.1 and 8.2 graphically present the scaling exponents for component concentrations as functions of parameters that were varied during testing. The first plot in each subsection displays the values of " $a$ " from all grid points (see Section 6.2), with different color plotting symbols used to display the " $a$ " values for the two $B S$ s. Two other plots in each subsection display subsets of the " $a$ " values in the first plot. Specifically, the second plot ( $B S=$ Typical) and third plot $(B S=$ High $)$ in each subsection show the values of " $a$ " as functions of selected combinations of $K V$ and $C V$. Different plotting symbols and colors are used to show the sensitivity of " $a$ " values to changes in $K V$ and $C V$. The lowest $K V$ values plotted are associated with the color red, the middle $K V$ values are associated with the color blue, and the highest $K V$ value plotted is associated with the color green. The lowest $C V$ values use diamond plotting symbols, the middle $C V$ values use triangle plotting symbols, and the largest $C V$ values use square plotting symbols. All three figures in each subsection exclude " $a$ " values that have a jet velocity in the 43 -inch tank or 120-inch tank that is outside the range of either of those velocities in the experimental data. For consistency, the same combinations of $K V$ and $C V$ values are used in the plots presented in this section as are used in the PPPs presented in Section 7.4 and Appendix D. These combinations are listed in Table 7.10 and correspond to the target $(K V, C V)$ combinations.

Note that some of the plots in Sections 8.1 and 8.2 include negative " $a$ " values. A negative scaling exponent indicates a lower velocity in the 120 -inch tank than in the 43 -inch tank is required to achieve the same component concentration. A positive scaling exponent indicates a higher velocity in the 120 -inch tank than in the 43-inch tank is required to achieve the same component concentration. 


\subsection{Scaling Exponent Results Using Models for Component Concentrations as Functions of the Test Parameters with Up to a Cubic Polynomial Dependence on Jet Velocity}

This section presents scaling exponent $(a)$ results for SS, sand, and $\mathrm{ZrO}_{2}$ concentrations. The results are presented as plots of " $a$ " versus mixer-jet pump velocity of the 43 -inch tank $\left(U_{43}\right)$ using the Section 7.1 models developed with polynomial dependence on $U$. It was not possible to apply the EP approach and calculate scaling exponents for gibbsite concentrations because the models for both the 43-inch tank and the 120-inch tank do not depend on $U$.

The following subsections graphically present the scaling exponents for concentrations of SS (Section 8.1.1), sand (Section 8.1.2), and $\mathrm{ZrO}_{2}$ (Section 8.1.3) as functions of parameters that were varied during testing. The scaling exponents in these subsections were calculated using the models in Section 7.1.

\subsubsection{Scaling Exponent Results for Stainless Steel Concentrations Using Models with Up to a Cubic Polynomial Dependence on Jet Velocity}

Figure 8.1 shows " $a$ " values associated with SS concentrations for all grid points, with the plotting symbols identifying the $B S$. These " $a$ " values were calculated using the SS concentration models in Table 7.2. Figure 8.1 shows that as $U_{43}$ increases, the " $a$ " values decrease for both base simulant types. Along the top edge of the plotted points, the " $a$ " values always correspond to $B S=$ Typical. For both $B S \mathrm{~s}$, the " $a$ " values for SS concentration are in the range $[-0.195,0.771]$, but the majority fall within the range $[-0.1,0.2]$. For $B S=$ Typical the range of " $a$ " values is $[-0.195,0.771]$ while for $B S=$ High the range is $[-0.136,0.688]$. The range of " $a$ " values for a given $U_{43}$ differs by $0.3-0.5$. This is because of the impact of the other parameters in the model $(K V, C V$, and $B N)$. To examine the impact of other parameters on the " $a$ " values for SS concentrations, Figure 8.2 and Figure 8.3 plot the " $a$ " values for $B S=$ Typical (Figure 8.2) and $B S=$ High (Figure 8.3) with different $U_{43}, K V$, and $C V$ settings. 


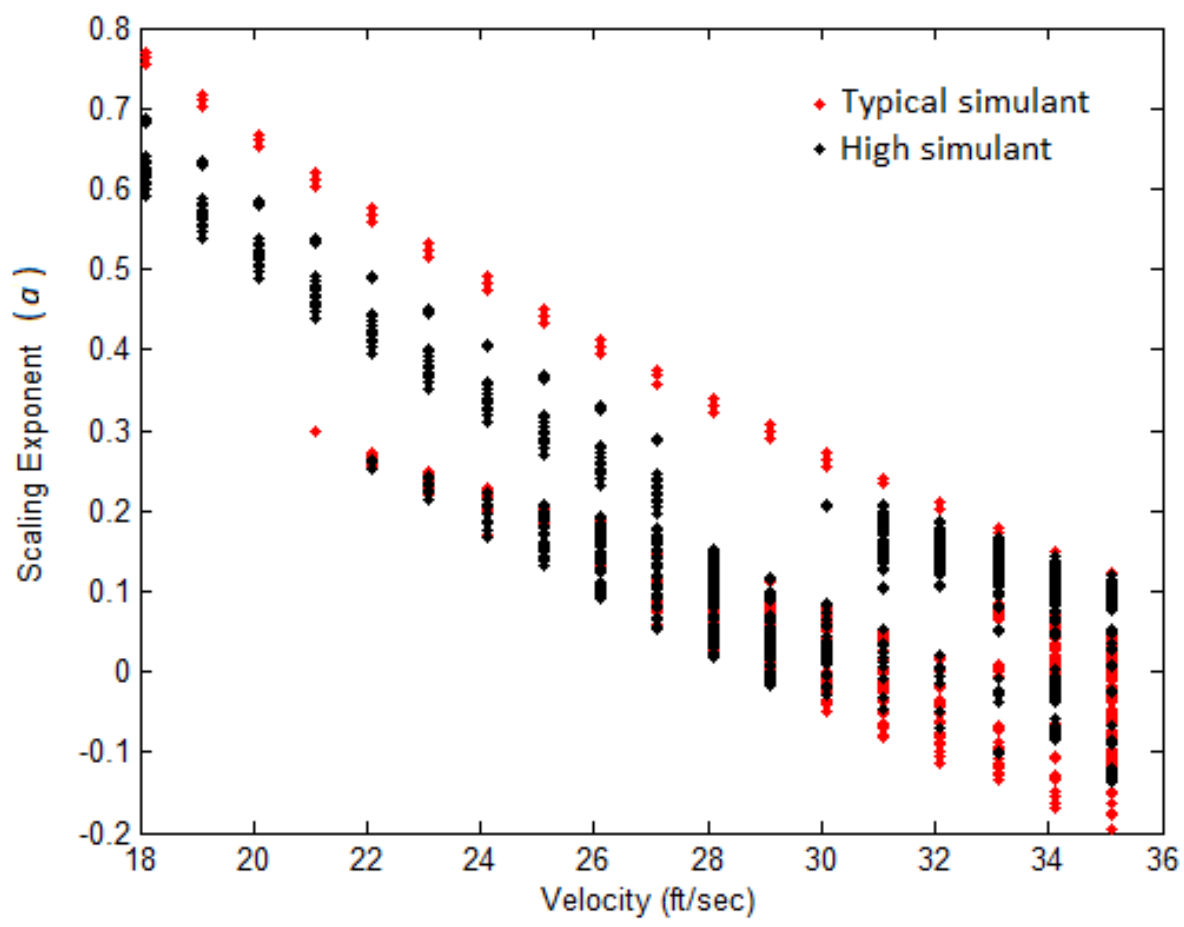

Figure 8.1. Scaling Exponent ( $a$ ) Values for Stainless Steel ( $y$-axis) versus Mixer-Jet Nozzle Velocity in the 43-inch Tank ( $x$-axis) Using the Models in Table 7.2. Observations are identified by type of base simulant.

The following observations are made from Figure 8.2 regarding the sensitivity of " $a$ " values for SS concentrations to changes in the parameters $U_{43}, K V$, and $C V$ when $B S=$ Typical

- The " $a$ " values tend to decrease as $U_{43}$ increases. This tendency of " $a$ " values to decrease with increasing $U_{43}$ is weakest when $K V=6.1$.

- Values of " $a$ " in the approximate range $0.3-0.8$ occur only for $K V=10.7$ and $C V=11.3$. Further, values of " $a$ " larger than $\sim 0.1$ occur only for $K V=2.6$ and 10.7 .

- For $K V=10.7 \mathrm{cSt}$ and $C V=11.3 \mathrm{ft} / \mathrm{sec}$ (green squares) with $U_{43}$ in the range $28-36 \mathrm{ft} / \mathrm{sec}$, there are points with both the smallest and largest " $a$ " values seen for a given $U_{43}$, which have different $B N$ values. Batches 0 (pre-transfer) and 1 achieve the larger " $a$ " values for a given $U_{43}$ in the range $28-36 \mathrm{ft} / \mathrm{sec}$ while batches 3,4 , and 5 result in smaller " $a$ " values in this same range. For batch 2 , the value of " $a$ " depends on the given $U_{43}$ with smaller values being achieved in the $30.5-32.5 \mathrm{ft} / \mathrm{sec}$ range. Thus, the scaling exponent decreases with increasing $B N$ at this set of conditions. 


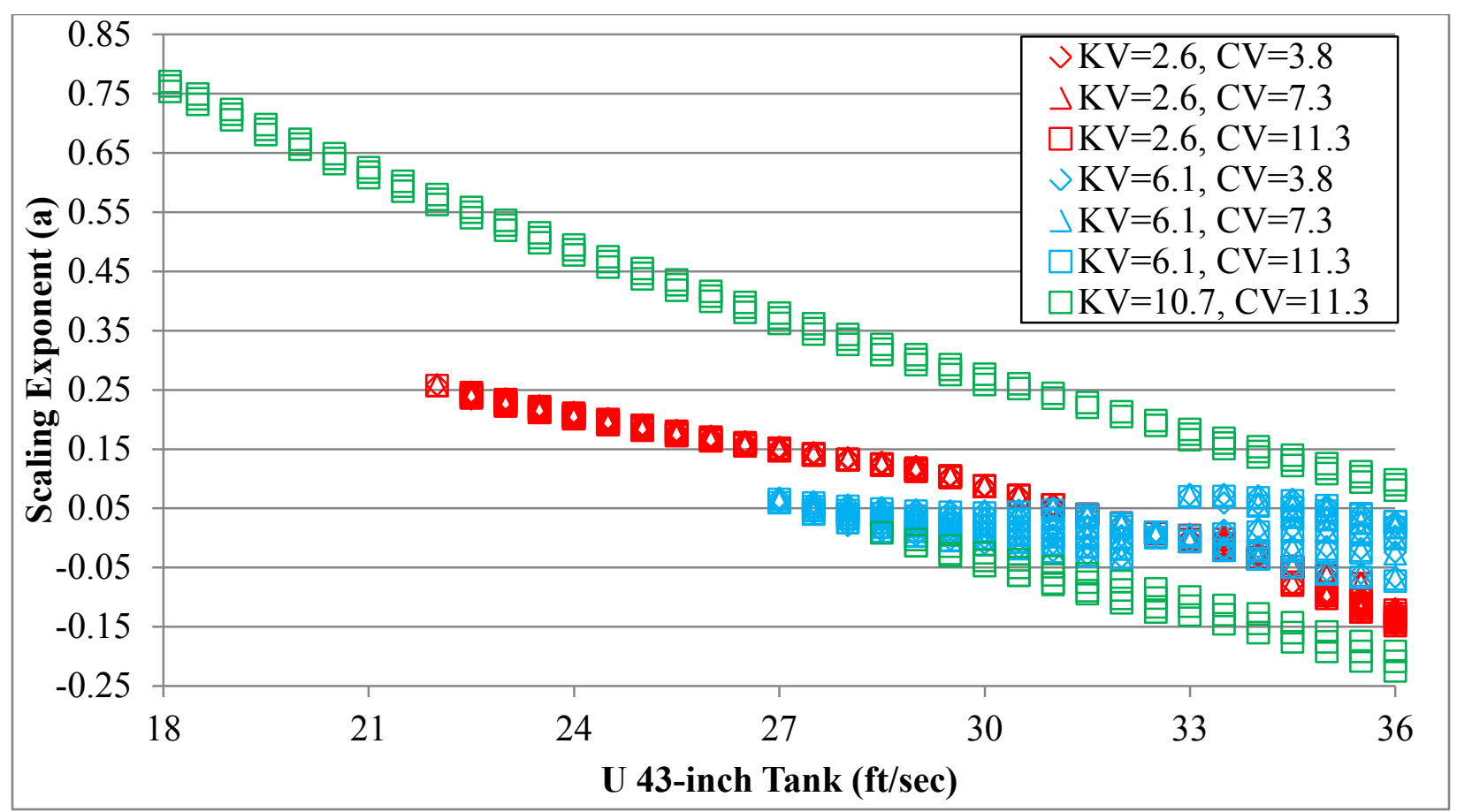

Figure 8.2. Scaling Exponent ( $a$ ) Values for Stainless Steel ( $y$-axis) versus Mixer-Jet Nozzle Velocity in the 43-inch Tank ( $x$-axis) with $B S=$ Typical and Various Combinations of $K V$ and $C V$ Using the Models in Table 7.2 (For Information Only)

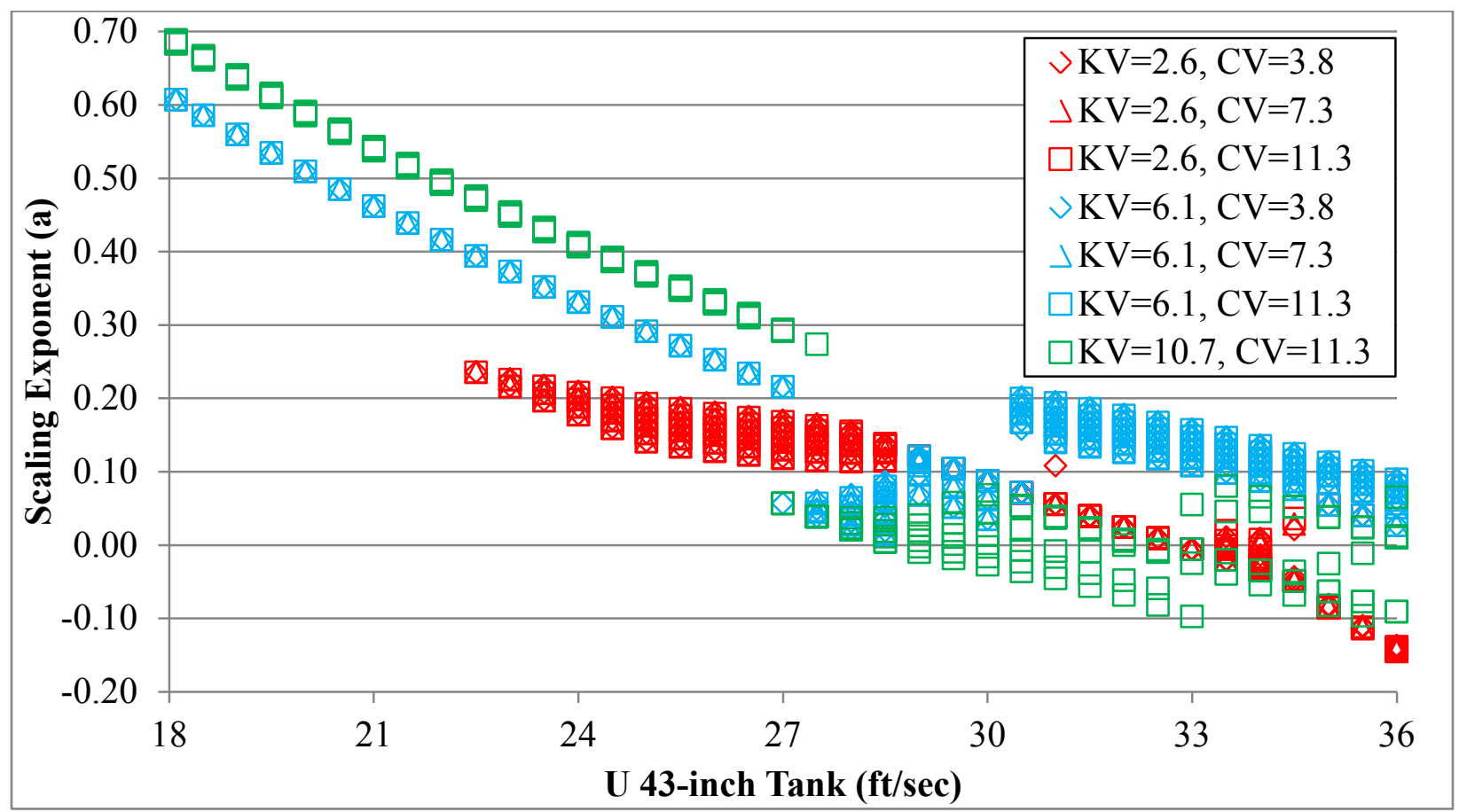

Figure 8.3. Scaling Exponent ( $a$ ) Values for Stainless Steel ( $y$-axis) versus Mixer-Jet Nozzle Velocity in the 43-inch Tank ( $x$-axis) with $B S=$ High and Various Combinations of $K V$ and $C V$ Using the Models in Table 7.2 (For Information Only) 
- The sensitivity of " $a$ " values to $C V$ appears to be minimal because most of the different plotting symbols of the same color are grouped together. There is no spread in " $a$ " values for the different $C V$ values when $K V=6.1 \mathrm{cSt}$; rather, the spread in the " $a$ " values is due to differences in $B N$. The range of " $a$ " values is still fairly narrow $[-0.1,0.1]$ for the different $B N$ values.

- The same plotting symbols being plotted close to each other for some combinations of parameter values occurs because multiple $B N$ values do not change " $a$ " very much.

The following observations are made from Figure 8.3 regarding the sensitivity of " $a$ " values for SS concentrations to changes in the parameters $U_{43}, K V$, and $C V$ when $B S=$ High.

- The " $a$ " values tend to decrease as $U_{43}$ increases. This tendency of " $a$ " values to decrease with increasing $U_{43}$ is weakest when $K V=6.1$.

- Values of " $a$ " in the approximate range $0.3-0.7$ occur for $K V=6.1$ and 10.7 cSt. For $U_{43}$ in the range $18-26 \mathrm{ft} / \mathrm{sec}$, " $a$ " increases with increasing $K V$.

- For $U_{43}$ in the range 29-36 ft/sec, the sensitivity of " $a$ " to $K V$ is less clear. However, at the highest $U_{43}$ values, the highest " $a$ " values tend to occur when $K V=6.1$.

- The sensitivity of " $a$ " values to $C V$ appears to be minimal because most of the different plotting symbols of the same color are grouped together.

- The same plotting symbols being plotted close to each other for some combinations of parameter values occurs because multiple $B N$ values do not change " $a$ " very much.

\subsubsection{Scaling Exponent Results for Sand Concentrations Using Models with Up to a Cubic Polynomial Dependence on Jet Velocity}

Figure 8.4 shows " $a$ " values associated with sand concentrations for all grid points, with the plotting symbols identifying the $B S$. These " $a$ " values were calculated using the sand concentration models in Table 7.3. Figure 8.4 shows that as $U_{43}$ increases, " $a$ " values decrease for both base simulant types. For both $B S \mathrm{~s}$, the " $a$ " values for SS concentration are in the range $[-0.052,0.345]$, but the majority fall within the range $[-0.1,0.2]$. For $B S=$ Typical the range of " $a$ " values is $[-0.052,0.262]$ while for $B S=$ High the range is $[-0.052,0.345]$. The range of " $a$ " values for a given $U_{43}$ differs by $0.025-0.15$. This is because of the impact of the other parameters in the model $(K V, C V$, and $B N)$. To examine the impact of other parameters on the " $a$ " values for sand concentrations, Figures 8.5 and 8.6 plot the " $a$ " values for $B S=$ Typical (Figure 8.5) and $B S=$ High (Figure 8.6) with different $U_{43}, K V$, and $C V$ settings. 


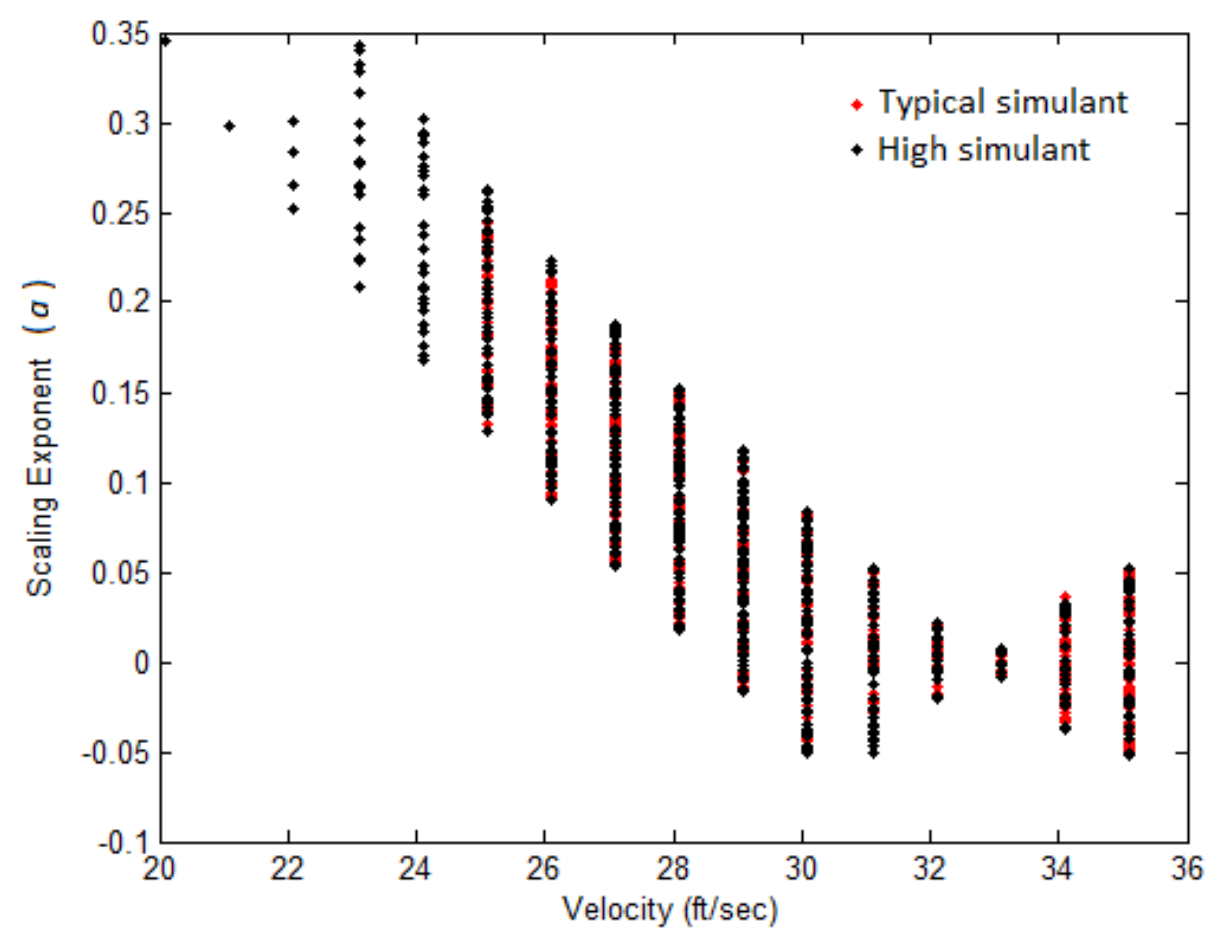

Figure 8.4. Scaling Exponent ( $a$ ) Values for Sand ( $y$-axis) versus Mixer-Jet Nozzle Velocity in the 43-inch Tank (x-axis) Using the Models in Table 7.3. Observations are identified by type of base simulant.

The following observations are made from Figure 8.5 regarding the sensitivity of " $a$ " values for sand concentrations to changes in the parameters $U_{43}, K V$, and $C V$ when $B S=$ Typical.

- The " $a$ " values tend to decrease as $U_{43}$ increases.

- For $C V=3.8 \mathrm{ft} / \mathrm{sec}$ with $K V=2.6$ and $6.1 \mathrm{cSt}$ (red and blue diamonds), values of " $a$ " are relatively large (in the range $0.15-0.27$ ) when $U_{43}$ is in the range $22-27 \mathrm{ft} / \mathrm{sec}$.

- For $U_{43}$ in the range 26-33 ft/sec, the scaling exponents tend to increase with $K V$. For a given $U_{43}$ value, the green squares $(K V=10.7 \mathrm{cSt})$ tend to have larger scaling exponents than the blue squares $(K V=6.1 \mathrm{cSt})$, which tend to have larger scaling exponents than the red squares $(K V=$ $2.6 \mathrm{cSt})$. Similarly for a given $U_{43}$ value, the blue triangles $(K V=6.1 \mathrm{cSt})$ tend to have higher scaling exponents than the red triangles $(K V=2.6 \mathrm{cSt})$. Thus, in the $U_{43}$ range of $26-33 \mathrm{ft} / \mathrm{sec}$, the " $a$ " values tend to increase with increasing $K V$.

- The plotting symbols with the same color and shape ( $K V$ and $C V$ combination) for a given $U_{43}$ represent the effect of $B N$ on " $a$ ". In some cases the symbols are plotted close to each other (e.g., blue diamonds, blue triangles, and blue squares), while in other cases the symbols have some spread (e.g., red diamonds, red triangles, and green squares). Hence, $B N$ impacts the scaling exponents for sand concentrations differently depending on the combination of $K V$ and $C V$. In general, as $B N$ increases, so does " $a$ " for the same combination of $K V$ and $C V$. However, the amount of the increase in " $a$ " depends on the values of $K V$ and $C V$. 


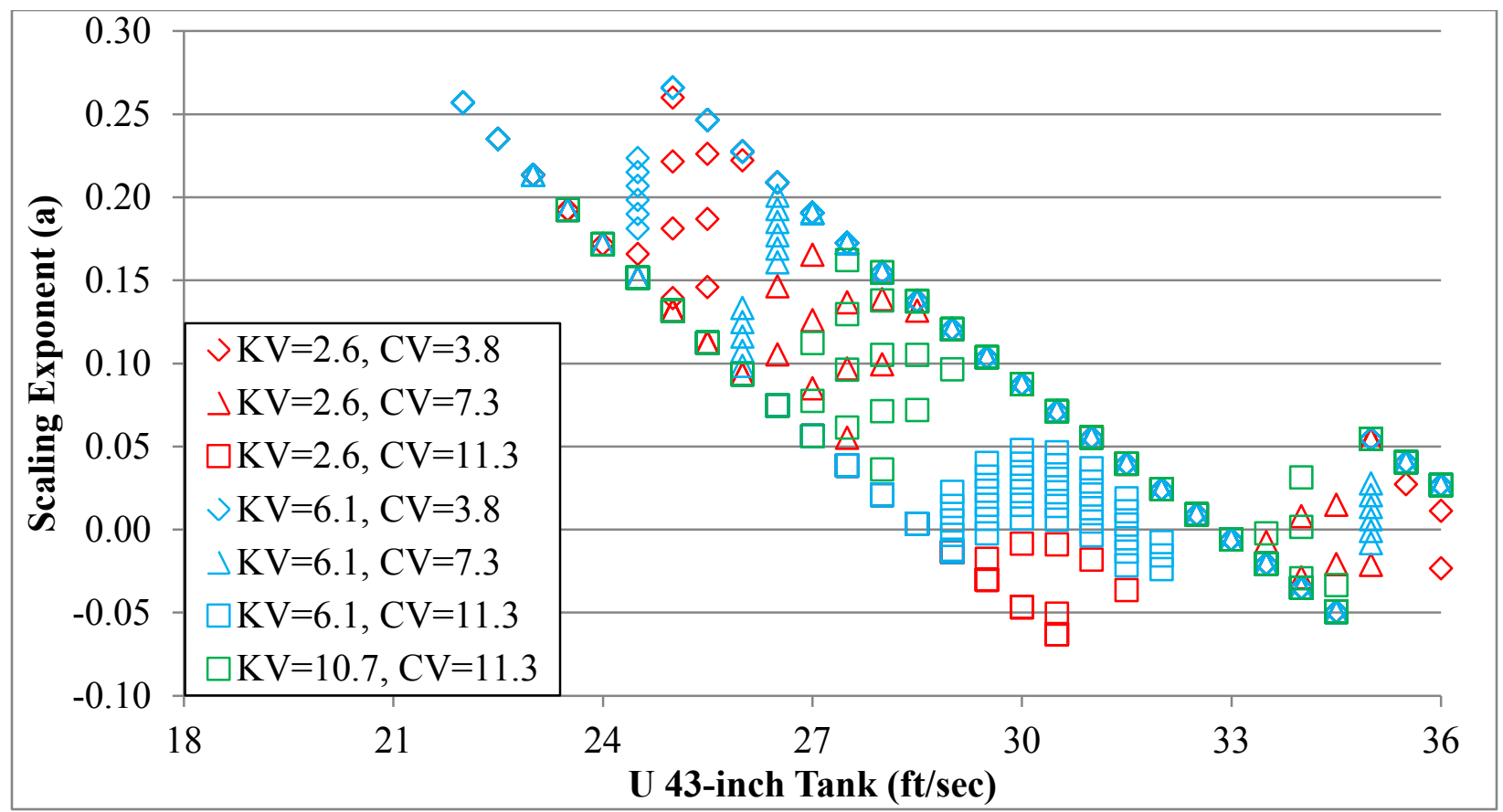

Figure 8.5. Scaling Exponent ( $a$ ) Values for Sand ( $y$-axis) versus Mixer-Jet Nozzle Velocity in the 43-inch Tank ( $x$-axis) with $B S=$ Typical and Various Combinations of $K V$ and $C V$ Using the Models in Table 7.3 (For Information Only)

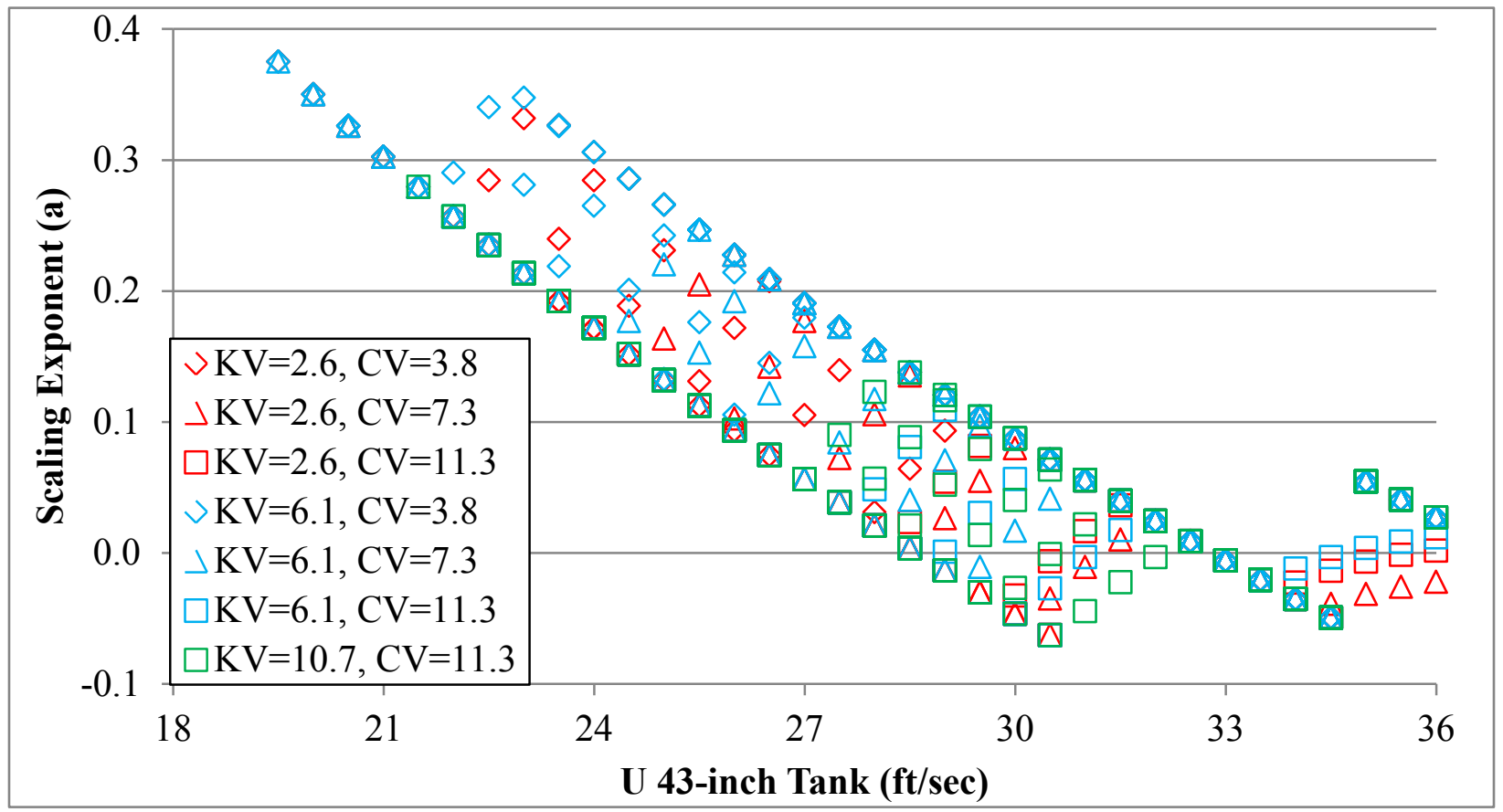

Figure 8.6. Scaling Exponent ( $a$ ) Values for Sand ( $y$-axis) versus Mixer-Jet Nozzle Velocity in the 43-inch Tank ( $x$-axis) with $B S=$ High and Various Combinations of $K V$ and $C V$ Using the Models in Table 7.3 (For Information Only) 
The following observations are made from Figure 8.6 regarding the sensitivity of " $a$ " values for sand concentrations to changes in the parameters $U_{43}, K V$, and $C V$ when $B S=$ High.

- The " $a$ " values tend to decrease as $U_{43}$ increases.

- There is a lot of overlap of symbols with different $K V$ values and $C V$ values, which results because of the several interaction terms in the models for the 43-inch and 120-inch tanks (see Table 7.3).

- In general, " $a$ " values tend to increase as $B N$ increases. There appears to be a narrow transition region (about $2 \mathrm{ft} / \mathrm{sec}$ wide) where the value of " $a$ " increases for a time as $U_{43}$ increases. The location of the transition region appears to depend on the values of $K V, C V$, and $B N$ with the transition occurring sooner (at smaller values of $U_{43}$ ) as $B N$ increases. The order of which symbols transition first appear to remain fairly constant for a given $B N$ with the transition order (from the transition happening from smallest to largest $U_{43}$ ) being blue diamonds, red diamonds, blue triangles, red triangles, green squares, blue squares, and finally red squares. Thus, it appears that the transition region is more greatly impacted by $C V$ than $K V$.

\subsubsection{Scaling Exponent Results for $\mathrm{ZrO}_{2}$ Concentrations Using Models with Up to a Cubic Polynomial Dependence on Jet Velocity}

Figure 8.7 shows " $a$ " values associated with $\mathrm{ZrO}_{2}$ concentrations for all grid points, with the plotting symbols identifying the $B S$. These " $a$ " values were calculated using the $\mathrm{ZrO}_{2}$ concentration models in Table 7.4. For both $B S \mathrm{~s}$, the " $a$ " values for $\mathrm{ZrO}_{2}$ are in the range $[-0.182,0.788]$, but the majority fall within the range $[-0.1,0.4]$. For $B S=$ Typical the range of " $a$ " values is $[-0.122,0.788]$ while for $B S=$ High the range is shifted downward and shrinks slightly, [-0.182, 0.652]. Figure 8.7 shows that as $U_{43}$, increases, the scaling exponent decreases for both base simulant types. The range of " $a$ " values for a given $U_{43}$ differs by $\sim 0.4$. This is because of the impact of the other parameters in the model $(K V, C V$, and $B N$ ). To examine the impact of other parameters on the scaling exponent $\mathrm{ZrO}_{2}$ concentrations, Figures 8.8 and 8.9 plot the " $a$ " values for $B S=$ Typical (Figure 8.8) and $B S=$ High (Figure 8.9) with different $U_{43}, K V$, and $C V$ settings. 


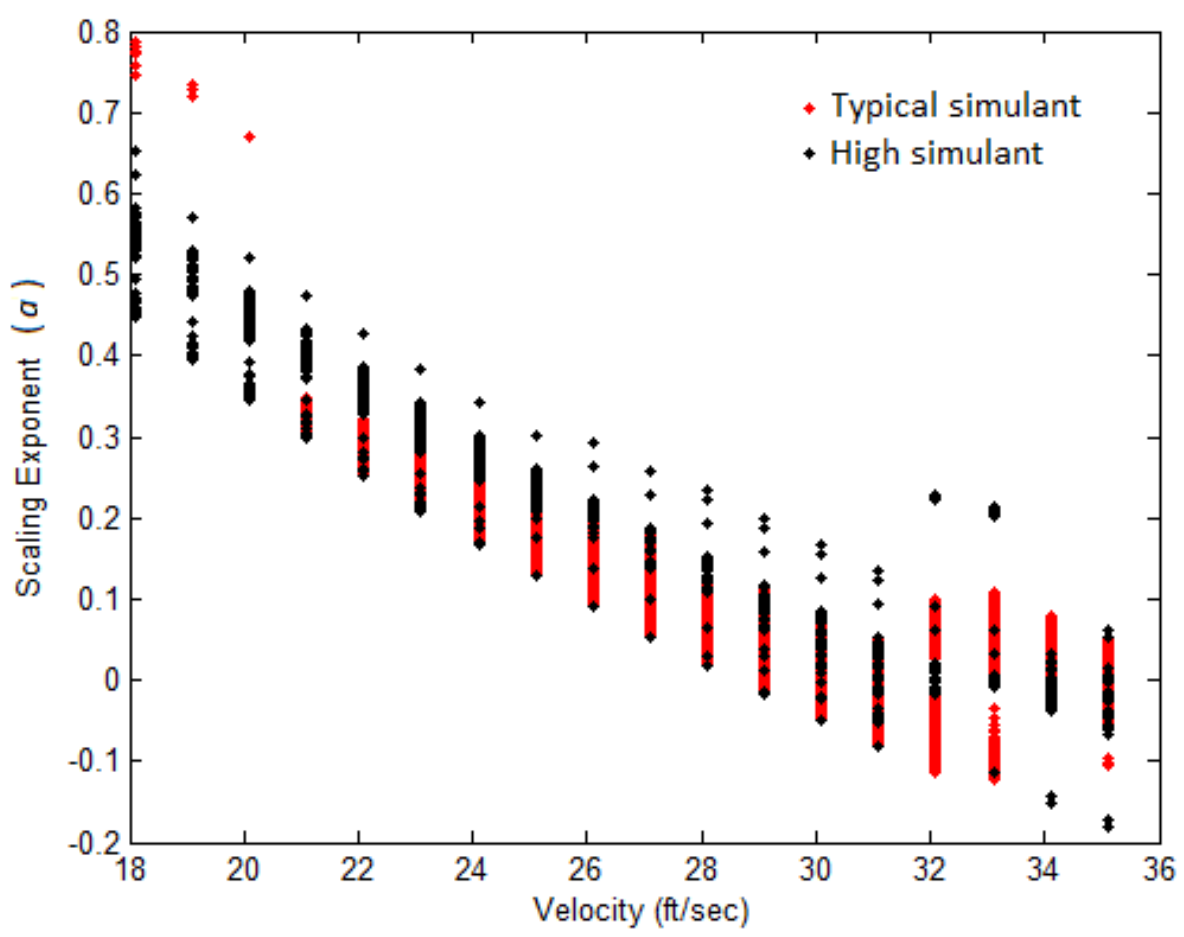

Figure 8.7. Scaling Exponent ( $a$ ) Values ( $y$-axis) versus Mixer-Jet Nozzle Velocity in the 43-inch Tank ( $x$-axis) for $\mathrm{ZrO}_{2}$ Using the Models in Table 7.4. Observations are identified by type of base simulant.

The following observations are made from Figure 8.8 regarding the sensitivity of " $a$ " values for $\mathrm{ZrO}_{2}$ concentrations to changes in the parameters $U_{43}, K V$, and $C V$ when $B S=$ Typical.

- The " $a$ " values tend to decrease as $U_{43}$ increases.

- For the $U_{43}$ range of 20-31.5 ft/sec, the largest " $a$ " values occur when $K V=2.6 \mathrm{cSt}$ (red symbols). This suggests that " $a$ " values tend to increase as $K V$ decreases.

- $\quad C V$ and $U_{43}$ appear to have an interactive effect on " $a$ " in the $U_{43}$ range of 31-35 ft/sec. For $U_{43}<$ $33 \mathrm{ft} / \mathrm{sec}$, the red squares $(C V=11.3 \mathrm{ft} / \mathrm{sec})$ tend to be below the red triangles $(C V=7.3 \mathrm{ft} / \mathrm{sec})$, which are below the red diamonds $(C V=3.8 \mathrm{ft} / \mathrm{sec})$. The ordering is reversed for $U_{43}>33 \mathrm{ft} / \mathrm{sec}$. A similar pattern occurs for the blue shapes $(K V=6.1 \mathrm{cSt})$ except the ordering reverses around $32.5 \mathrm{ft} / \mathrm{sec}$.

- For the $U_{43}$ range of 18-21 ft/sec, there appears to be large impact of $B N$ on the " $a$ " when $K V=10.7 \mathrm{cSt}$ and $C V=11.3 \mathrm{ft} / \mathrm{sec}$, with a difference of $\sim 0.35$ between the green squares for a given $U_{43}$. For other combinations of $K V$ and $C V$ the spread in " $a$ " values for a given $U_{43}$ value is generally small, indicating little effect of $B N$ on " $a$ ". 


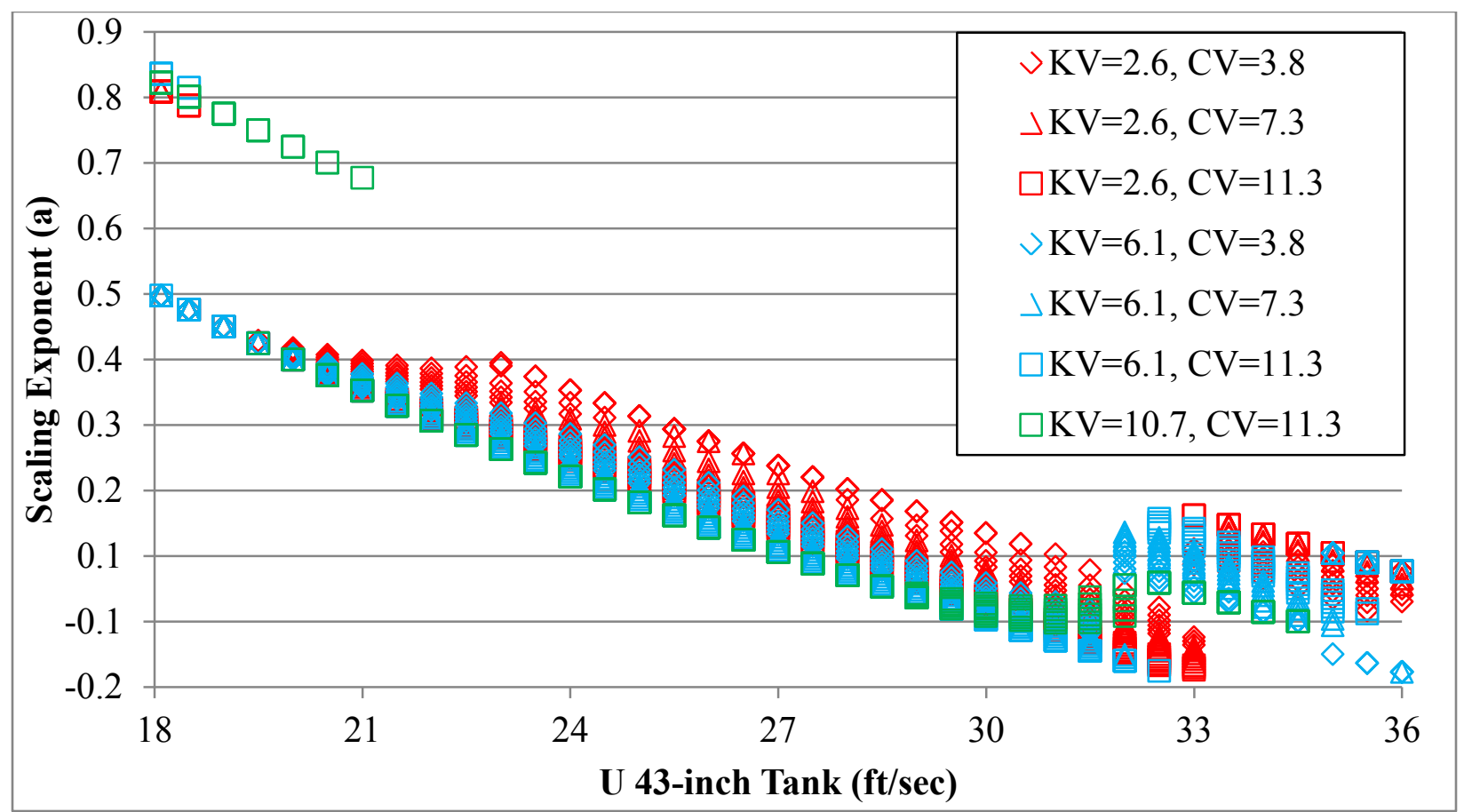

Figure 8.8. Scaling Exponent ( $a$ ) Values for $\mathrm{ZrO}_{2}$ ( $y$-axis) versus Mixer-Jet Nozzle Velocity in the 43-inch Tank ( $x$-axis) with $B S=$ Typical and Various Combinations of $K V$ and $C V$ Using the Models in Table 7.4 (For Information Only)

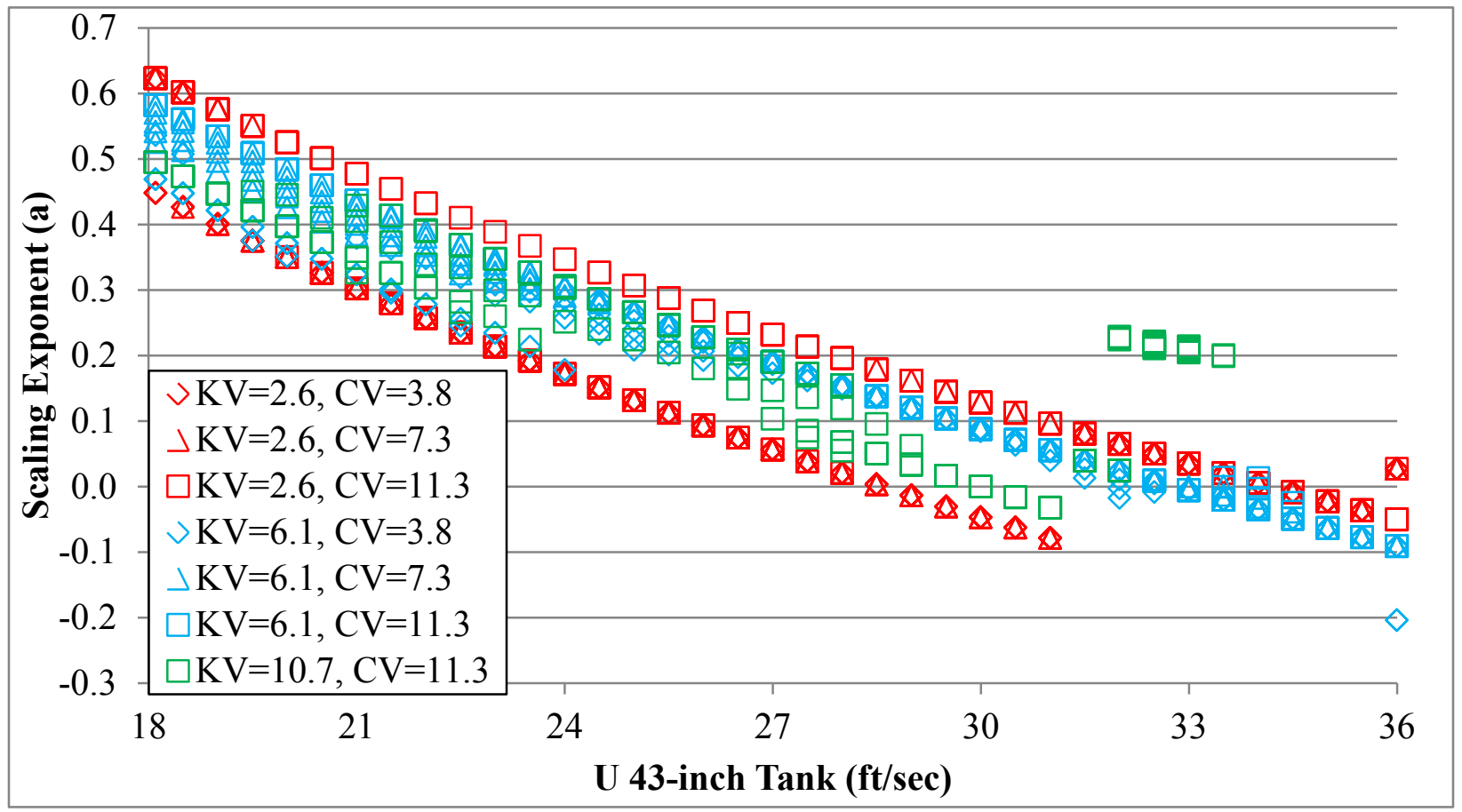

Figure 8.9. Scaling Exponent ( $a$ ) Values for $\mathrm{ZrO}_{2}$ ( $y$-axis) versus Mixer-Jet Nozzle Velocity in the 43-inch Tank ( $x$-axis) with $B S=$ High and Various Combinations of $K V$ and $C V$ Using the Models in Table 7.4 (For Information Only) 
The following observations are made from Figure 8.9 regarding the sensitivity of " $a$ " values for $\mathrm{ZrO}_{2}$ concentrations to changes in the parameters $U_{43}, K V$, and $C V$ when $B S=$ High.

- The " $a$ " values tend to decrease as $U_{43}$ increases. The main exception is for the case of $K V=10.7$ $\mathrm{cSt}$ and $C V=11.3 \mathrm{ft} / \mathrm{sec}$ for $U_{43}$ from $31-32 \mathrm{ft} / \mathrm{sec}$.

- The red points $(K V=2.6 \mathrm{cSt})$ vary greatly with the widest range of " $a$ " values (around 0.15$)$ for a given $U_{43}$, meaning $B N$ has a large effect on " $a$ " values at this value of $K V$. For $K V=2.6$, values of " $a$ " tend to increase with increasing $B N$. However, for other values of $K V$ and most values of $U_{43}$, there is no impact of $B N$ on " $a$ " values.

- The blue points show less separation of the different plotting symbols when $U_{43}>27 \mathrm{ft} / \mathrm{sec}$. The blue shapes (diamonds, triangles, and squares) tend to overlap, showing no dependence of " $a$ " values on $C V$ for $K V=6.1 \mathrm{cSt}$ for this range of $U_{43}$ values.

- For the case of $K V=10.7 \mathrm{cSt}$ and $C V=11.3 \mathrm{ft} / \mathrm{sec}$ (green squares) there is an increase of $0.2 \mathrm{in}$ the " $a$ " values for $U_{43}$ values in the range $30-33 \mathrm{ft} / \mathrm{sec}$.

\subsection{Scaling Exponent Results Using Models for Component Concentrations as Functions of the Test Parameters with a Power-Law Dependence on Jet Velocity}

This section presents scaling exponent $(a)$ results for SS and sand concentrations. The results are presented as plots of " $a$ " versus $U_{43}$ using the Section 7.2 models having dependence on $\ln (U)$ and possibly $[\ln (U)]^{2}$. The models for gibbsite and $\mathrm{ZrO}_{2}$ in the 120 -inch tank do not depend on $U$, so it was not possible to apply the EP approach and calculate scaling exponents for gibbsite and $\mathrm{ZrO}_{2}$.

The following subsections graphically present the scaling exponents for concentrations of SS (Section 8.2.1) and sand (Section 8.2.2) as functions of parameters that were varied during testing. The scaling exponents in these subsections were calculated using the models in Section 7.2.

\subsubsection{Scaling Exponent Results for Stainless Steel Concentrations Using Models Related to a Power-Law Dependence on Jet Velocity}

Figure 8.10 shows " $a$ " values associated with SS concentrations for all grid points, with the plotting symbols identifying the $B S$. These " $a$ " values were calculated using the SS concentration models in Table 7.6. Figure 8.10 shows that as $U_{43}$ increases, the " $a$ " values decrease for both base simulant types. For both $B S$ s, the " $a$ " values for SS concentration are in the range $[-0.187,0.321]$, but the majority fall within the range $[-0.1,0.3]$. For $B S=$ Typical the range of " $a$ " values is $[-0.187,0.321]$ while for $B S=$ High the range is $[-0.067,0.319]$. The range of " $a$ " values for a given $U_{43}$ differs by $0.3-0.5$. This is because of the impact of the other parameters in the model $(K V, C V$, and $B N)$. Figure 8.10 also shows differences in the pattern of the scaling exponents versus base simulant at the lowest and highest $U_{43}$ values, which may be due to the nature of the interaction terms in the model. To examine the impact of other parameters on the scaling exponent for SS concentrations, Figures 8.11 and 8.12 plot the " $a$ " values for $B S=$ Typical (Figure 8.11) and $B S=$ High (Figure 8.12) with different $U_{43}, K V$, and $C V$ settings. 


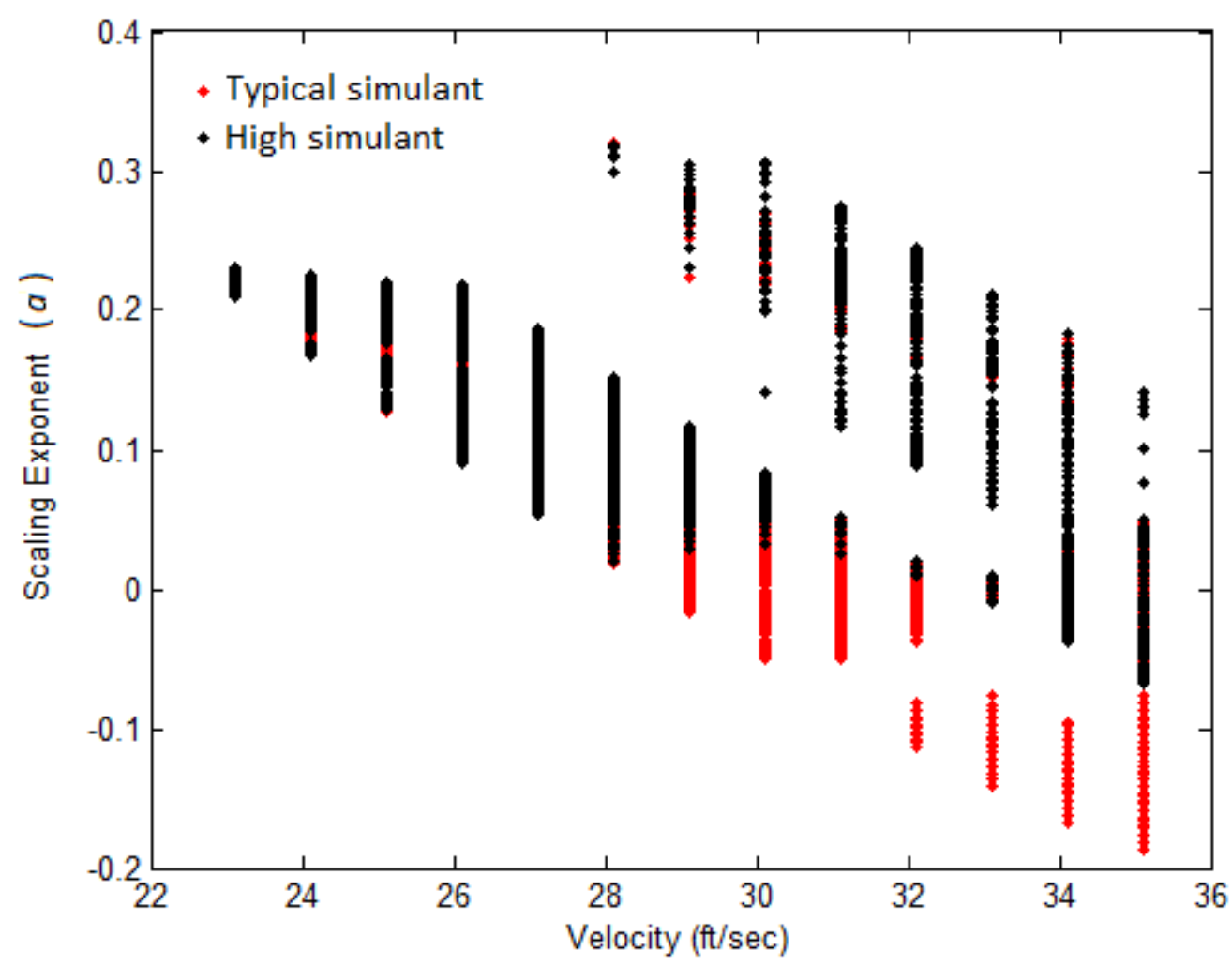

Figure 8.10. Scaling Exponent (a) Values (y-axis) versus Mixer-Jet Nozzle Velocity in the 43-inch Tank $(x$-axis) for Stainless Steel using the Models in Table 7.6. Observations are identified by type of base simulant.

The following observations are made from Figure 8.11 regarding the sensitivity of " $a$ " values for SS concentrations to changes in the parameters $U_{43}, K V$, and $C V$ when $B S=$ Typical.

- Symbols tend to group by color, indicating that " $a$ " values are generally more sensitive to changes in $K V$ than changes in $C V$.

- For $K V=2.6 \mathrm{cSt}$, the " $a$ " values tend to decrease as $U_{43}$ increases.

- For $K V=6.1 \mathrm{cSt}$ (blue symbols), the " $a$ " values mostly vary in the range $[-0.05,0.05]$ and do not vary much with increasing $U_{43}$. All three shapes of the blue symbols are tightly packed (within 0.05 ), indicating little effect of $C V$ on " $a$ " when $K V=6.1 \mathrm{cSt}$.

- For the case $K V=2.6 \mathrm{cSt}$ and $C V=11.3 \mathrm{ft} / \mathrm{sec}$ (red squares) there are some $U_{43}$ values (30-33.5 $\mathrm{ft} / \mathrm{sec}$ ) where the " $a$ " values increase significantly (by over 0.15 ) for $B N \geq 4$ as compared to the other batches.

- The largest " $a$ " values, in the range [0.13, 0.27], occur for $K V=2.7 \mathrm{cSt}$ (red symbols).

- In general, for the same color (blue or red), the diamonds are below the triangles which are below the squares indicating a slight increase in " $a$ " for increasing $C V$ for a given $B N$.

- As $B N$ increases, there is a slight increase in scaling exponent values except in the case of $K V=$ $2.6 \mathrm{cSt}$ and $C V=11.3 \mathrm{ft} / \mathrm{sec}$ where there is a large $(0.15)$ increase in the " $a$ " value between batches 3 and 4 . 


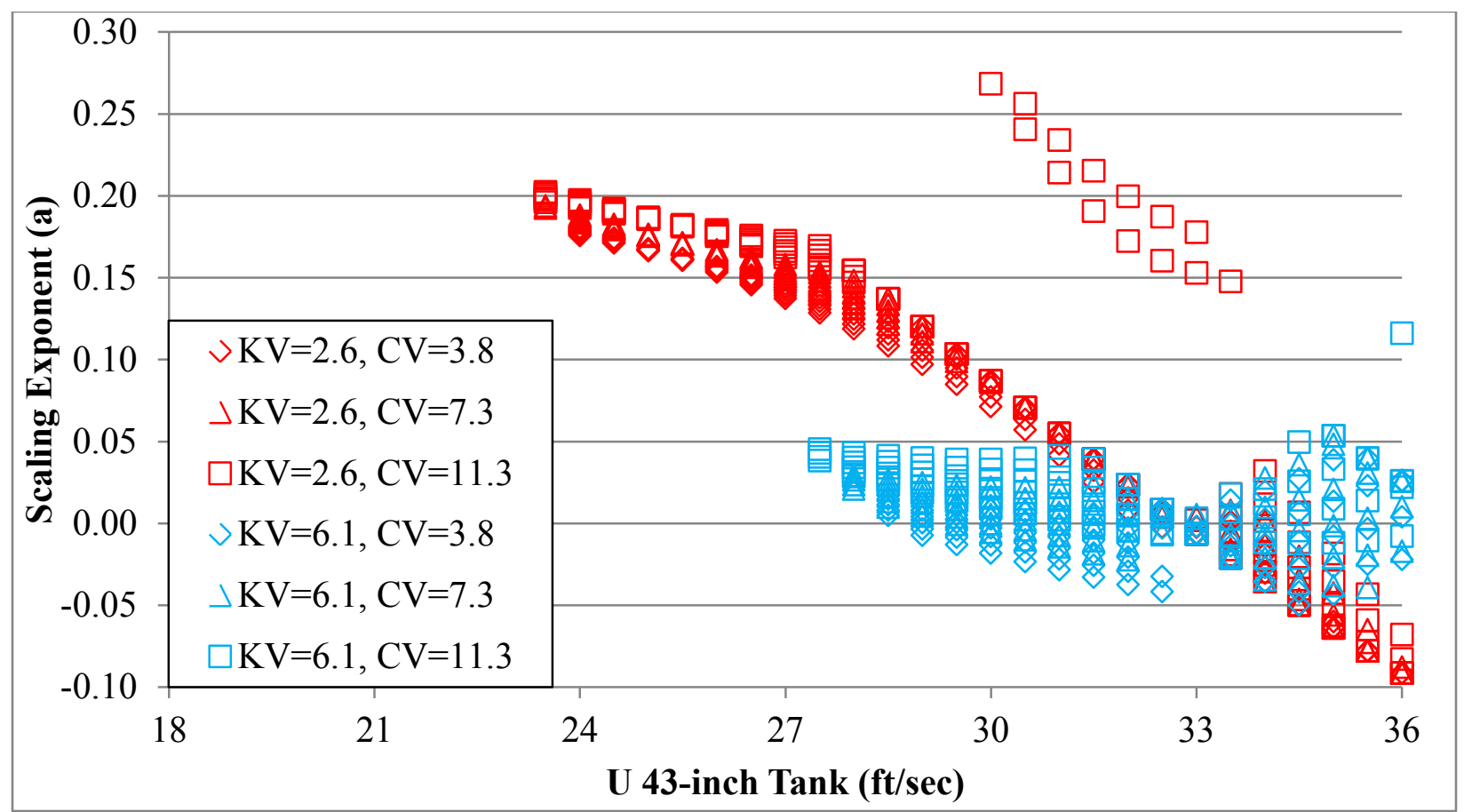

Figure 8.11. Scaling Exponent (a) Values ( $y$-axis) versus Mixer-Jet Nozzle Velocity in the 43-inch Tank ( $x$-axis) for Stainless Steel using the Models in Table 7.6 for $B S=$ Typical (For Information Only)

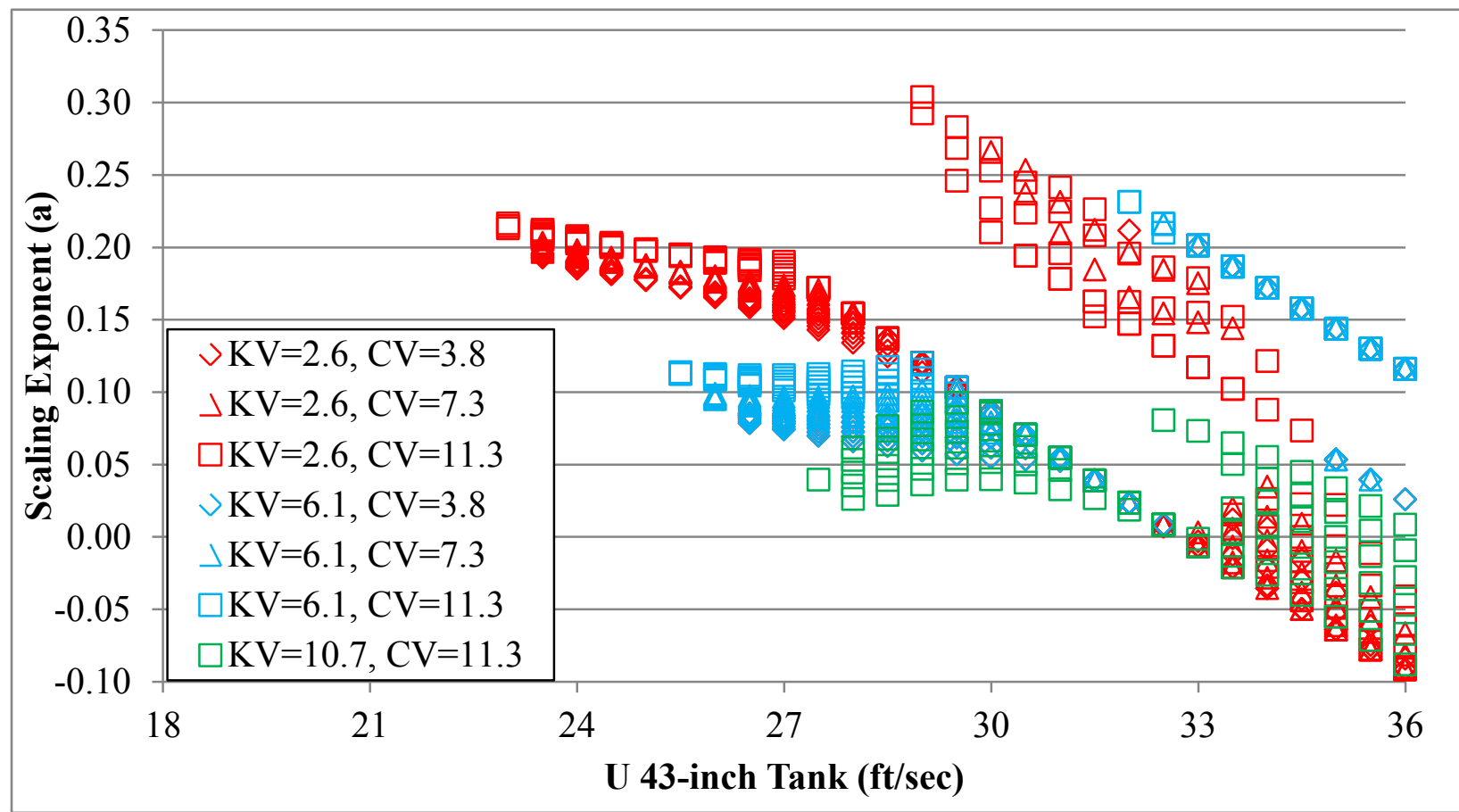

Figure 8.12. Scaling Exponent (a) Values (y-axis) versus Mixer-Jet Nozzle Velocity in the 43-inch Tank ( $x$-axis) for Stainless Steel using the Models in Table 7.6 for $B S=$ High (For Information Only) 
- The case of $K V=10.7 \mathrm{cSt}$ and $C V=11.3 \mathrm{ft} / \mathrm{sec}$ is not represented in Figure 8.11. All of the grid points involving this combination of $K V$ and $C V$ had $U_{120}$ values (calculated to give EP) in the range 22 to $26 \mathrm{ft} / \mathrm{sec}$, which is less than the smallest mixer-jet velocity tested in the 120-inch tank. Thus, the " $a$ " values calculated for $K V=10.7 \mathrm{cSt}$ and $C V=11.3 \mathrm{ft} / \mathrm{sec}$ were excluded.

The following observations are made from Figure 8.12 regarding the sensitivity of " $a$ " values for SS concentrations to changes in the parameters $U_{43}, K V$, and $C V$ when $B S=\mathrm{High}$.

- There are distinct color groupings of symbols, indicating that $K V$ is important in determining the scaling exponent. Within each group, the " $a$ " values tend to decrease with increasing $U_{43}$, although the decrease is not the same for every group. This indicates an interaction between $K V$ and $\ln \left(U_{43}\right)$.

- For $K V=2.6 \mathrm{cSt}$ (red symbols), the " $a$ " values fall into three groups, one for $U_{43}$ in the range 23$29.5 \mathrm{ft} / \mathrm{sec}$, another for $U_{43}$ in the range $29.5-34 \mathrm{ft} / \mathrm{sec}$, and the third for $U_{43}$ in the range 33-36 $\mathrm{ft} / \mathrm{sec}$. In the first group, all the different symbol shapes are very close, indicating little effect of $C V$ on " $a$ " values. In the second group (i) the " $a$ " values tend to decrease with increasing $U_{43}$, and (ii) for a given value of $U_{43}$ the plotting symbols of different shapes and the same shapes are intermixed, indicating smaller effects of $C V$ and $B N$. For the third group we again see (i) the " $a$ " values tend to decrease with increasing $U_{43}$, and (ii) for a given value of $U_{43}$ the plotting symbols of different shapes and the same shapes are intermixed, indicating smaller effects of $C V$ and $B N$.

- For $K V=6.1 \mathrm{cSt}$, the " $a$ " values fall in three groups similar to what was seen for $K V=2.7 \mathrm{cSt}$. However, there is less variability in the " $a$ " values for the second and third groups with larger $U_{43}$ values.

- For the case of $K V=10.7 \mathrm{cSt}$ and $C V=11.3 \mathrm{ft} / \mathrm{sec}$ (green squares), the " $a$ " values are in the range $[-0.1,0.1]$ and generally appear to decrease as $U_{43}$ increases. For a given value of $U_{43}$, the range in the green squares is sufficient to indicate a moderate effect of " $a$ " increasing with increasing $B N$.

\subsubsection{Scaling Exponent Results for Sand Concentrations Using Models Related to a Power-Law Dependence on Jet Velocity}

Figure 8.13 shows " $a$ " values associated with sand concentrations for all grid points, with the plotting symbols identifying the $B S$. These " $a$ " values were calculated using the sand concentration models in Table 7.7. Figure 8.13 shows that as $U_{43}$ increases, the " $a$ " values decrease for both base simulant types. For both $B S \mathrm{~s}$, the " $a$ " values for SS concentration are in the range [-0.052, 0.343], but the majority fall within the range $[-0.05,0.30]$. For $B S=$ Typical the range of " $a$ " values is $[-0.054,0.261]$ while for $B S=$ High the range is $[-0.052,0.343]$. The range of " $a$ " values for a given $U_{43}$ differs by $0.025-0.15$. This is because of the impact of the other parameters in the model $(K V, C V$, and $B N)$. To examine the impact of other parameters on the scaling exponent for sand concentrations, Figures 8.14 and 8.15 plot the " $a$ " values for $B S=$ Typical (Figure 8.14) and $B S=$ High (Figure 8.15) with different $U_{43}, K V$, and $C V$ settings. 


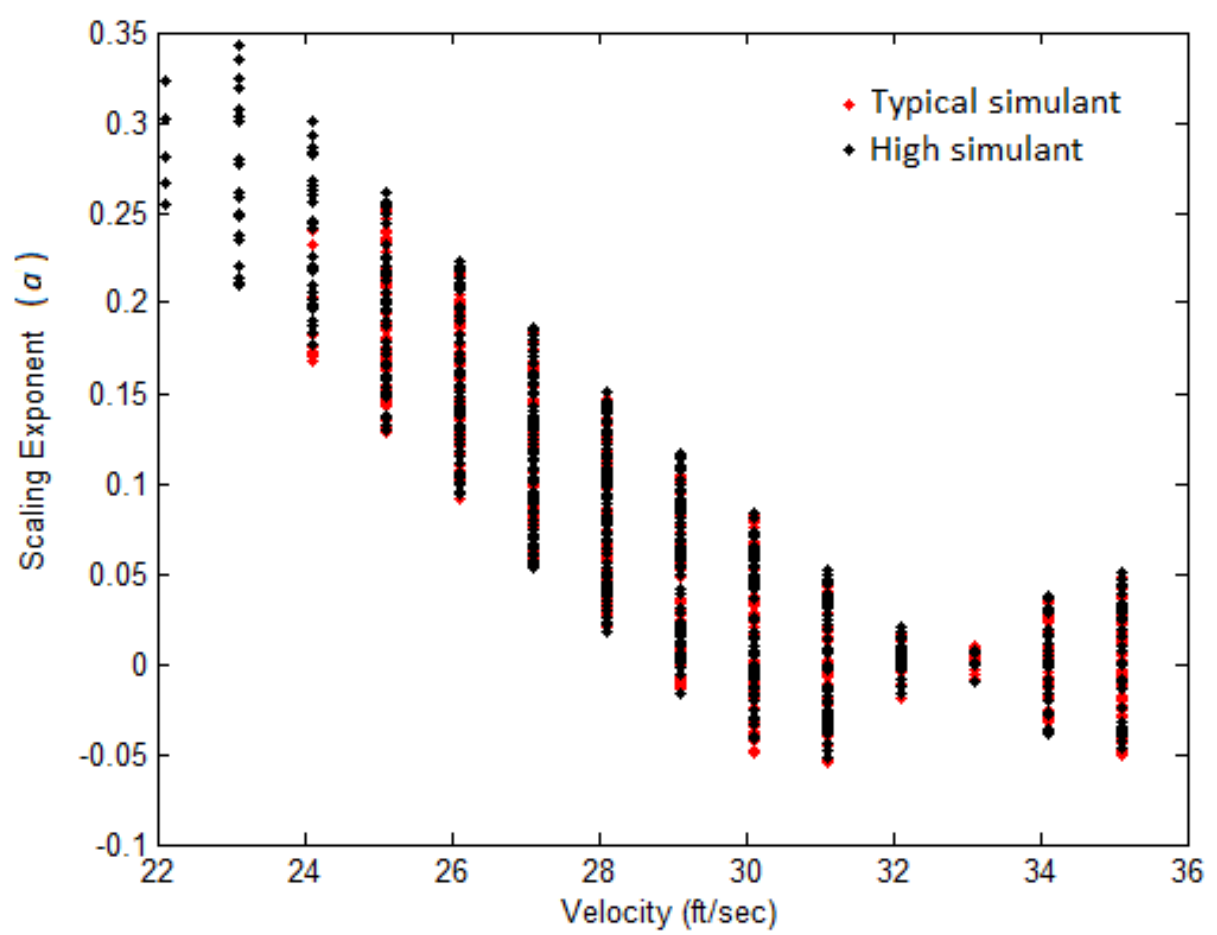

Figure 8.13. Scaling Exponent (a) Values ( $y$-axis) versus Mixer-Jet Nozzle Velocity in the 43-inch Tank ( $x$-axis) for Sand Using the Models in Table 7.7. Observations are identified by type of base simulant.

The following observations are made from Figure 8.14 regarding the sensitivity of " $a$ " values for sand concentrations to changes in the parameters $U_{43}, K V$, and $C V$ when $B S=$ Typical.

- The " $a$ " values tend to decrease as $U_{43}$ increases. Several combinations of a plotting symbol shape and color (e.g., blue triangles, green squares, and red triangles) form noticeable groups, with each group displaying curvature in " $a$ " as a decreasing function of $U_{43}$.

- $K V$ appears to affect " $a$ " values because the shapes separate by color. For a given $U_{43}$, the green squares $(K V=10.7 \mathrm{cSt})$ have larger " $a$ " values than the blue squares $(K V=6.1 \mathrm{cSt})$ for $U_{43}<31$ $\mathrm{ft} / \mathrm{sec}$. Similarly, the blue triangles $(K V=6.1 \mathrm{cSt})$ have larger " $a$ " values than the red triangles $(K V=2.6 \mathrm{cSt})$ for $U_{43}<32 \mathrm{ft} / \mathrm{sec}$. Finally, the blue diamonds $(K V=6.1 \mathrm{cSt})$ have larger " $a$ " values than the red diamonds $(K V=2.6 \mathrm{cSt})$. Hence, the scaling exponents for sand concentration tend to increase with increasing $K V$ for smaller mixer-jet pump velocities $\left(U_{43}<31\right.$ $\mathrm{ft} / \mathrm{sec})$. For larger mixer-jet pump velocities $\left(U_{43}>33 \mathrm{ft} / \mathrm{sec}\right)$ the scaling exponents for sand concentration tend to decrease with increasing $K V$.

- The plotting symbols with the same color and shape ( $K V$ and $C V$ combination) for a given $U_{43}$ represent the effect of $B N$ on " $a$ ". In some cases the symbols are plotted close to each other (e.g., blue diamonds, blue triangles, and blue squares), while in other cases the symbols have some spread (e.g., red diamonds, red triangles, and green squares). Hence, $B N$ impacts the scaling exponents for sand concentration differently, depending on the combination of $K V$ and $C V$. However, generally " $a$ " increases as $B N$ increases for the same $K V$ and $C V$ combination. 


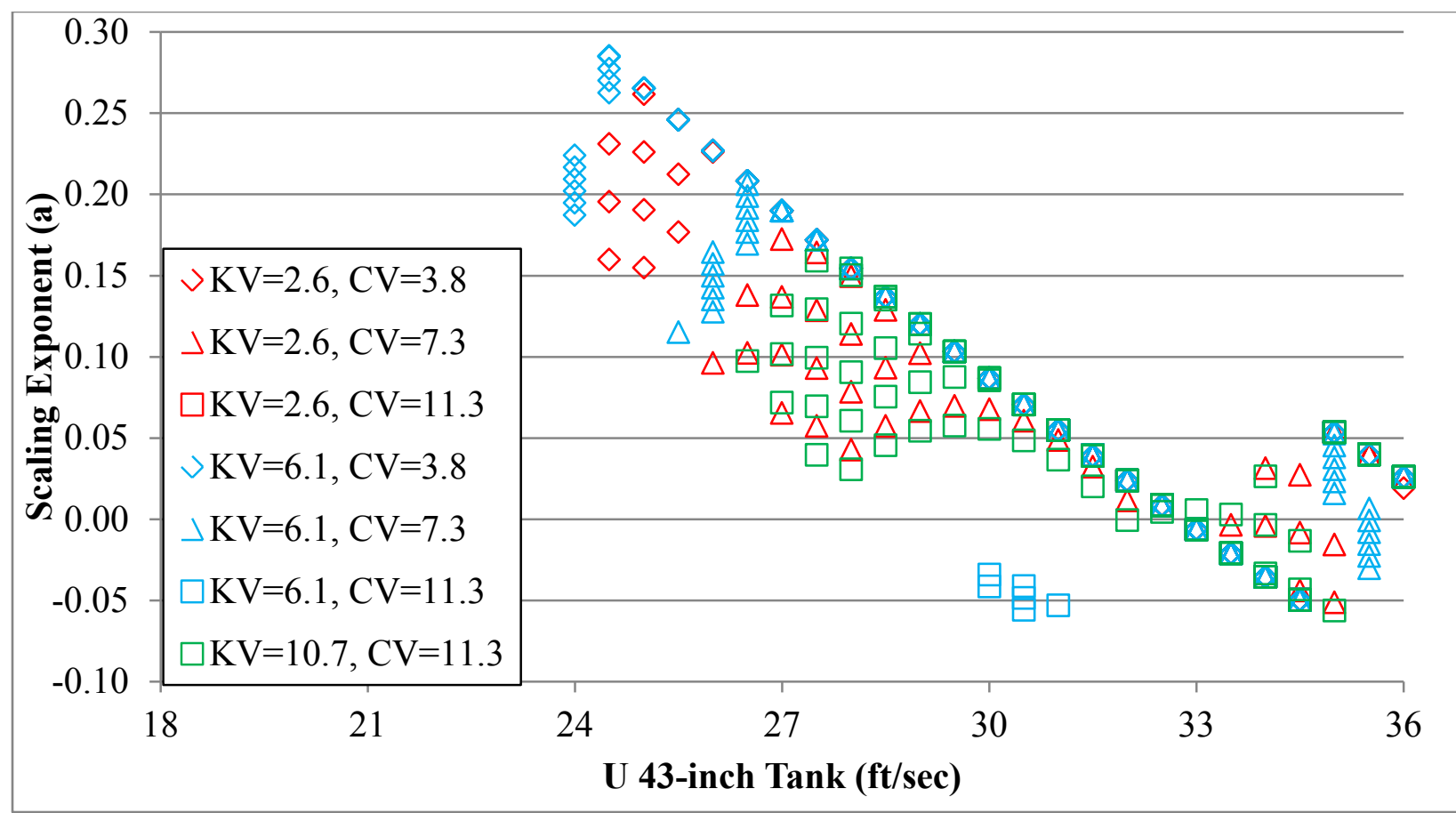

Figure 8.14. Scaling Exponent ( $a$ ) Values ( $y$-axis) versus Mixer-Jet Nozzle Velocity in the 43-inch Tank ( $x$-axis) for Sand Using the Models in Table 7.7 for $B S=$ Typical (For Information Only)

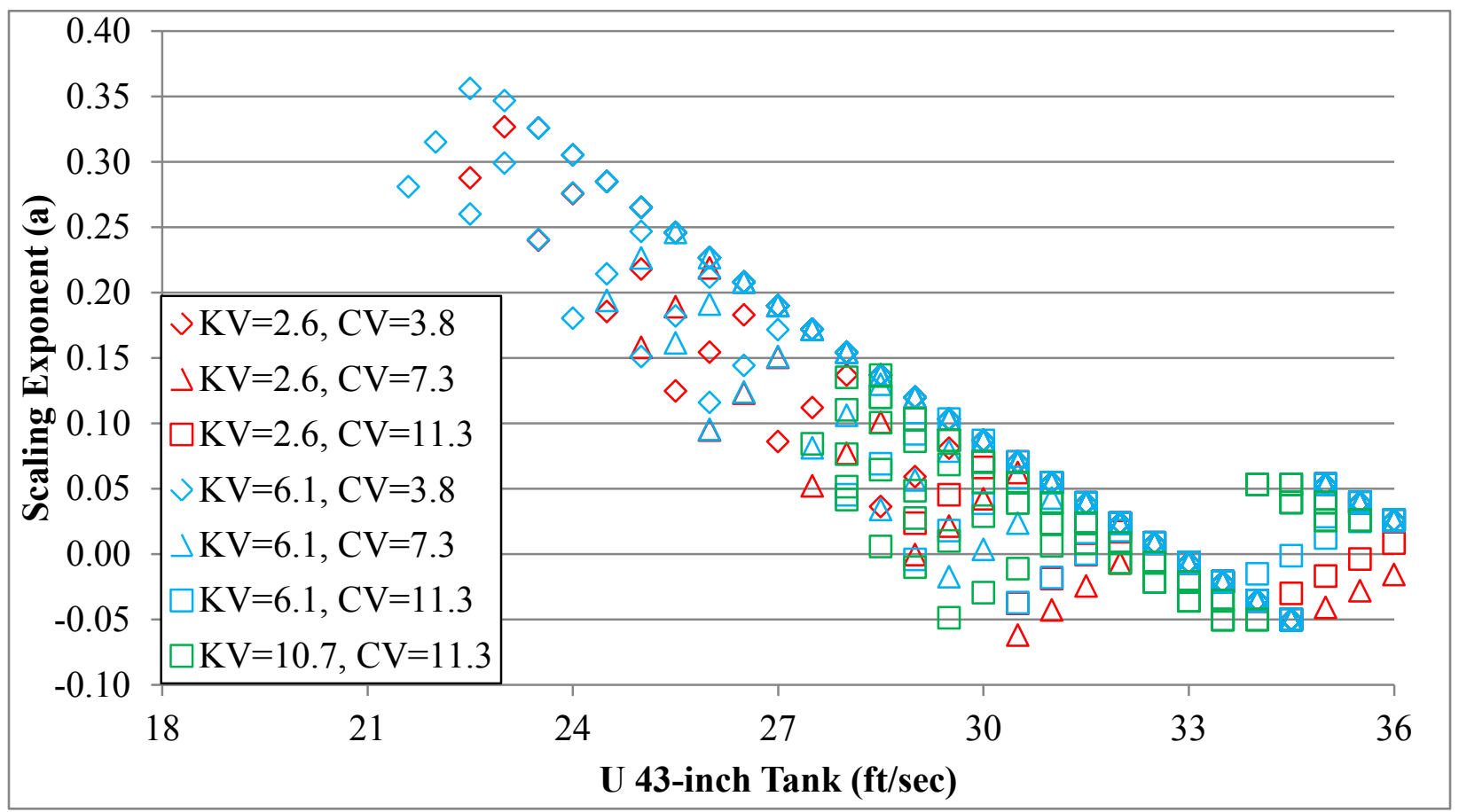

Figure 8.15. Scaling Exponent (a) Values ( $y$-axis) versus Mixer-Jet Nozzle Velocity in the 43-inch Tank ( $x$-axis) for Sand Using the Models in Table 7.7 for BS = High (For Information Only) 
The following observations are made from Figure 8.15 regarding the sensitivity of " $a$ " values for sand concentrations to changes in the parameters $U_{43}, K V$, and $C V$ when $B S=$ High.

- The " $a$ " values tend to increase over a small range of $U_{43}$ values (about $2 \mathrm{ft} / \mathrm{sec}$ wide), and then decrease as $U_{43}$ increases until they reach higher mixer-jet pump velocities $\left(U_{43}>34 \mathrm{ft} / \mathrm{sec}\right)$ when " $a$ " again increases with increasing $U_{43}$. The location ( $U_{43}$ values) associated with the initial increase in scaling exponent is dependent on $B N$.

- The " $a$ " values appear to have a strong dependence on $C V$. The lowest $C V$ value of $3.8 \mathrm{ft} / \mathrm{sec}$ has the highest " $a$ " values, which are in the range $[-0.05,0.35]$. The middle $C V$ value $7.3 \mathrm{ft} / \mathrm{sec}$ has intermediate " $a$ " values, which are in the range $[-0.06,0.25]$. Finally, the highest $C V$ value of $11.3 \mathrm{ft} / \mathrm{sec}$ has the smallest " $a$ " values, which are in the range $[-0.05,0.15]$. However, there is still quite a bit of overlap of " $a$ " values for these ranges. The narrower ranges of " $a$ " values as $C V$ increases is related to the ranges of $U_{43}$ values that resulted in a calculated $U_{120}$ value within its tested range. For $C V=3.8 \mathrm{ft} / \mathrm{sec}$, scaling exponents are calculated for $U_{43}>21 \mathrm{ft} / \mathrm{sec}$. For $C V$ $=7.3 \mathrm{ft} / \mathrm{sec}$, scaling exponents are calculated for $U_{43}>24 \mathrm{ft} / \mathrm{sec}$. For $C V=11.3 \mathrm{ft} / \mathrm{sec}$, scaling exponents are calculated for $U_{43}>27 \mathrm{ft} / \mathrm{sec}$.

- There appears to be an effect of $B N$ on whether an increase in $U_{43}$ results in increasing or decreasing " $a$ " values, although there is still a lot of overlap (especially in the case of the blue symbols where $K V=6.1 \mathrm{cSt}$ ). This suggests an interaction term between $B N$ and $K V$.

\subsection{Scaling Exponent Conclusions}

Table 8.1 summarizes the ranges of scaling exponents for SS and sand concentrations from Section 8.1 (based on models in Section 7.1) and Section 8.2 (based on models in Section 7.2). The scaling exponents for the Section 7.1 models (with up to cubic polynomial dependence on mixer-jet nozzle velocity, $U$ ) have much wider ranges than do the scaling exponents for the Section 7.2 models (based on $\ln (U)$ and possibly $[\ln (U)]^{2}$ dependence on $U$. Of particular note is that all scaling exponents for SS concentration (including all combinations of $B S, K V, C V$, and $B N$ ) are lower than 0.78 with the Section 7.1 models, but are lower than 0.33 with the Section 7.2 models. All scaling exponents for sand concentration (including both base simulants) are lower than 0.35 with both the Section 7.1 and Section 7.2 models.

Table 8.1. Summary of Scaling Exponent Ranges for SS and Sand Concentrations Based on the Models in Section 7.1 and Section 7.2

\begin{tabular}{llcc}
\hline & & \multicolumn{2}{c}{ Range of All Scaling Exponents } \\
\hline Component & BS & Section 7.1 Models & Section 7.2 Models \\
\hline SS & Typical & {$[-0.195,0.771]$} & {$[-0.187,0.321]$} \\
SS & High & {$[-0.136,0.688]$} & {$[-0.067,0.319]$} \\
Sand & Typical & {$[-0.052,0.262]$} & {$[-0.054,0.261]$} \\
Sand & High & {$[-0.052,0.345]$} & {$[-0.052,0.343]$} \\
\hline
\end{tabular}

The much larger scaling exponent values obtained using the SS concentration models in Section 7.1 apparently are related to modeling component concentrations using up to cubic dependence on $U$. The 
PPPs presented and discussed in Section 7.4.1 showed that the models for SS concentrations could significantly overpredict component concentrations in some cases. On the other hand, much smaller scaling exponent values are obtained using the Section 7.2 models for SS concentration, apparently due to modeling component concentrations as functions of $\ln (U)$ and possibly $[\ln (U)]^{2}$. The PPPs presented and discussed in Section 7.4.2 did not show the same tendency for the Section 7.2 models to overpredict SS concentrations.

Based on the PPPs in Section 7.4 and the scaling exponents discussed in Sections 8.1 and 8.2, it is concluded that the models in Section 7.2 are preferred for modeling component concentrations and for calculating scaling exponents using the EP approach discussed in Section 6.2 because the predictions for these models are more reasonable. The maximum scaling exponents calculated by the Section 7.2 models for SS and sand are within the [0.2-0.4] range envisioned by Lee (2012, p. 3-27). Also, the range of scaling exponent values calculated by the Section 7.2 models is consistent with values anticipated based on Figures 3.2 and 3.3. Hence, it is recommended that the range of scaling exponents identified using the Section 7.2 models be explored by WRPS to estimate full-scale, DST performance. It can be concluded that scaling exponent is a function of jet velocity and simulant make-up (e.g. $B S, K V$ ). Therefore all these factors must be considered when estimating full-scale performance. 


\subsection{References}

Adamson DJ, MR Poirier, and TJ Steeper. 2009. Demonstration Simulated Waste Transfers from Tank AY-102 to Hanford Waste Treatment Facility. SRNL-STI-2009-00717, Rev. 0, Savannah River National Laboratory, Savannah River Nuclear Solutions, Aiken, South Carolina.

Atkinson AC and AN Donev. 1992. Optimal Experimental Design. Oxford University Press, New York.

DuMouchel W and B Jones. 1994. "A Simple Bayesian Modification of D-optimal Designs to Reduce Dependence on an Assumed Model." Technometrics 36:37-47.

Greer DA. 2012. Tank Farm Approach to Develop Scaling Relationships Based on Scaled Tank Mixing and Transfer Results. RPP-PLAN-53454, Rev. 0, Washington River Protection Solutions, Richland, Washington.

Jensen J. 2011. SSMD Test Platform, Small Scale Mixing Demonstration, Sampling \& Batch Transfers Results Report. RPP-49740, EnergySolutions, Richland, Washington.

JMP. 2012. JMP Statistical Software, Version 10.0.0. SAS, Cary, North Carolina.

Lee KP. 2012. One System Waste Feed Delivery Mixing and Sampling Program System Performance Test Plan. RPP-PLAN-52623, Rev. 0, Washington River Protection Solutions, Richland, Washington.

Lee KP, GF Piepel, AE Holmes, and A Heredia-Langner. 2012a. Tank Farm Approach to Develop Scaling Relationships Based on Scaled Tank Mixing and Transfer Results. RPP-PLAN-53454, Rev. 1, Washington River Protection Solutions, Richland, Washington.

Lee KP, BE Wells, PA Gauglitz, and RA Sexton. 2012b. Waste Feed Delivery Mixing and Sampling Program Simulant Definition for Tank Farm Performance Testing. RPP-PLAN-51625, Rev. 0, Washington River Protection Solutions, Richland, Washington.

Myers RH and DC Montgomery. 1995. Response Surface Methodology: Process and Product Optimization Using Designed Experiments. John Wiley \& Sons, New York.

Wells BE, JL Huckaby JM Tingey, DE Kurath, SK Cooley, RC Daniel, LA Mahoney, CA Burns, KK Anderson, Y Onishi, and EC Buck. 2011. Hanford Waste Physical and Rheological Properties: Data and Gaps. PNNL-20646, Rev. 0, Pacific Northwest National Laboratory, Richland, Washington.

Weier DR and GF Piepel. 2003. Methodology for Adjusting and Normalizing Analyzed Glass Compositions. PNWD-3260 (WTP-RPT-049), Battelle-Pacific Northwest Division, Richland, Washington. 


\section{Appendix A}

\section{Mixing and Transfer Performance Tests and Resulting Concentrations of Gibbsite, Stainless Steel, Sand, and $\mathrm{ZrO}_{2}$ in Pre-Transfer and Batch Transfer Samples}




\section{Appendix A}

\section{Mixing and Transfer Performance Tests and Resulting Concentrations of Gibbsite, Stainless Steel, Sand, and $\mathrm{ZrO}_{2}$ in Pre-Transfer and Batch Transfer Samples}

Table A.1 lists for each test the planned (target) values as well as actual values (when available) of the test parameters $(U, B S, S V, C V)$, along with other relevant information about the test conditions (e.g., the actual kinematic viscosity, which is used subsequently in modeling the experimental results). Table A. 2 lists the concentrations (lb/gal slurry) of the four solids components (gibbsite, SS, sand, and $\mathrm{ZrO}_{2}$ ) for the pre-transfer samples and the five batch-transfer samples. Data are included for the 22 test combinations in Table 2.3 and the 4 test combinations in Table 2.4 that were performed in each of the 43inch and 120-inch tanks in the Small Scale Mixing Demonstration (SSMD) testing platform. The data file that served as the basis for data below was "SSMD_SP_Data_Review_Data_Package_RevD.xlsx", which was received from Pat Lee at WRPS on June 20, 2013.

Table A.1. Parameter Values for the 26 Test Combinations Performed in 43-inch and 120-inch Tanks

\begin{tabular}{|c|c|c|c|c|c|c|c|c|c|c|c|}
\hline $\begin{array}{l}\text { Test } \\
\text { No. }\end{array}$ & $\begin{array}{c}\text { Tank } \\
\text { Size } \\
\text { (in.) }\end{array}$ & $\begin{array}{c}B S \\
\text { (a) }\end{array}$ & $\begin{array}{c}\text { Target } \\
\text { Jet } \\
\text { Velocity } \\
\text { (ft/sec) }\end{array}$ & $\begin{array}{c}\text { Target } \\
\text { Dynamic } \\
\text { Viscosity } \\
\text { (cP) }\end{array}$ & $\begin{array}{c}\text { Target } \\
\text { Capture } \\
\text { Velocity } \\
\text { (ft/sec) }\end{array}$ & $\begin{array}{c}\text { Target } \\
\text { Density } \\
(\mathrm{g} / \mathrm{mL})\end{array}$ & $\begin{array}{c}\text { Average } \\
\text { Jet } \\
\text { Velocity }^{(\mathbf{b})} \\
\text { (ft/sec) }\end{array}$ & $\begin{array}{c}\text { Actual } \\
\text { Dynamic } \\
\text { Viscosity } \\
\text { (cP) }\end{array}$ & $\begin{array}{c}\text { Average } \\
\text { Capture } \\
\text { Velocity } \\
\text { (ft) } / \text { sec) }\end{array}$ & $\begin{array}{c}\text { Actual } \\
\text { Density } \\
\text { (g/mL) }\end{array}$ & $\begin{array}{c}\text { Actual } \\
\text { Kinematic } \\
\text { Viscosity } \\
\text { (cSt) }\end{array}$ \\
\hline 1 & 43 & $\mathrm{H}$ & 18.24 & 3.3 & 7.3 & 1.284 & 18.85 & 2.900 & 7.305 & 1.300 & 2.231 \\
\hline 2 & 43 & $\mathrm{H}$ & 26.05 & 3.3 & 7.3 & 1.284 & 27.54 & 2.200 & 7.346 & 1.255 & 1.753 \\
\hline 3 & 43 & $\mathrm{~T}$ & 22.14 & 3.3 & 7.3 & 1.284 & 22.50 & 3.300 & 7.339 & 1.290 & 2.558 \\
\hline 4 & 43 & $\mathrm{~T}$ & 33.87 & 3.3 & 7.3 & 1.284 & 34.14 & 2.600 & 7.550 & 1.305 & 1.992 \\
\hline 5 & 43 & $\mathrm{~T}$ & 33.87 & 3.3 & 7.3 & 1.284 & 36.04 & 2.400 & 7.385 & 1.310 & 1.832 \\
\hline 6 & 43 & $\mathrm{H}$ & 22.14 & 8 & 3.8 & 1.320 & 23.09 & 7.600 & 3.810 & 1.310 & 5.802 \\
\hline 7 & 43 & $\mathrm{H}$ & 22.14 & 8 & 3.8 & 1.320 & 23.67 & 6.800 & 3.829 & 1.310 & 5.191 \\
\hline 8 & 43 & $\mathrm{H}$ & 29.96 & 8 & 3.8 & 1.320 & 29.44 & 6.100 & 3.865 & 1.300 & 4.692 \\
\hline 9 & 43 & $\mathrm{H}$ & 18.24 & 8 & 7.3 & 1.320 & 18.18 & 7.600 & 7.390 & 1.300 & 5.846 \\
\hline 10 & 43 & $\mathrm{H}$ & 33.87 & 8 & 7.3 & 1.320 & 35.07 & 7.000 & 7.397 & 1.305 & 5.364 \\
\hline 11 & 43 & $\mathrm{H}$ & 26.05 & 8 & 11.3 & 1.320 & 26.96 & 9.000 & 10.430 & 1.315 & 6.844 \\
\hline 12 & 43 & $\mathrm{H}$ & 26.05 & 8 & 11.3 & 1.320 & 27.06 & 8.000 & 11.257 & 1.300 & 6.154 \\
\hline 13 & 43 & $\mathrm{~T}$ & 26.05 & 8 & 3.8 & 1.320 & 26.25 & 7.000 & 3.781 & 1.305 & 5.364 \\
\hline 14 & 43 & $\mathrm{~T}$ & 18.24 & 8 & 7.3 & 1.320 & 18.30 & 8.800 & 7.346 & 1.305 & 6.743 \\
\hline 15 & 43 & $\mathrm{~T}$ & 33.87 & 8 & 7.3 & 1.320 & 34.82 & 7.700 & 7.327 & 1.310 & 5.878 \\
\hline 16 & 43 & $\mathrm{~T}$ & 26.05 & 8 & 11.3 & 1.320 & 27.57 & 7.200 & 11.225 & 1.305 & 5.517 \\
\hline 17 & 43 & $\mathrm{~T}$ & 26.05 & 8 & 11.3 & 1.320 & 26.50 & 6.700 & 11.232 & 1.300 & 5.154 \\
\hline 18 & 43 & $\mathrm{H}$ & 18.24 & 14.6 & 11.3 & 1.370 & 18.14 & 12.000 & 11.081 & 1.350 & 8.889 \\
\hline 19 & 43 & $\mathrm{H}$ & 26.05 & 14.6 & 11.3 & 1.370 & 26.04 & 15.400 & 11.229 & 1.350 & 11.407 \\
\hline 20 & 43 & $\mathrm{H}$ & 33.87 & 14.6 & 11.3 & 1.370 & 32.24 & 11.500 & 10.899 & 1.350 & 8.519 \\
\hline 21 & 43 & $\mathrm{H}$ & 22.14 & 14.6 & 11.3 & 1.370 & 21.92 & 15.100 & 11.114 & 1.350 & 11.185 \\
\hline 22 & 43 & $\mathrm{~T}$ & 29.96 & 14.6 & 11.3 & 1.370 & 29.18 & 15.200 & 9.773 & 1.355 & 11.218 \\
\hline 23 & 43 & $\mathrm{~T}$ & 22.14 & 3.3 & 3.8 & 1.284 & 22.40 & 3.100 & 3.817 & 1.315 & 2.357 \\
\hline 24 & 43 & $\mathrm{~T}$ & 26.05 & 3.3 & 3.8 & 1.284 & 26.18 & 3.200 & 3.821 & 1.345 & 2.379 \\
\hline 25 & 43 & $\mathrm{~T}$ & 29.96 & 3.3 & 3.8 & 1.284 & 30.30 & 2.700 & 3.829 & 1.345 & 2.007 \\
\hline 26 & 43 & $\mathrm{H}$ & 26.05 & 3.3 & 3.8 & 1.284 & 26.18 & 3.200 & 3.797 & 1.335 & 2.397 \\
\hline
\end{tabular}


Table A.1. Parameter Values for the 26 Test Combinations Performed in 43-inch and 120-inch Tanks (cont.)

\begin{tabular}{|c|c|c|c|c|c|c|c|c|c|c|c|}
\hline $\begin{array}{l}\text { Test } \\
\text { No. }\end{array}$ & $\begin{array}{c}\text { Tank } \\
\text { Size } \\
\text { (in.) } \\
\end{array}$ & $\begin{array}{c}B S \\
\text { (a) } \\
\end{array}$ & $\begin{array}{c}\text { Target } \\
\text { Jet } \\
\text { Velocity } \\
\text { (ft/sec) } \\
\end{array}$ & $\begin{array}{c}\text { Target } \\
\text { Dynamic } \\
\text { Viscosity } \\
\text { (cP) } \\
\end{array}$ & $\begin{array}{c}\text { Target } \\
\text { Capture } \\
\text { Velocity } \\
\text { (ft/s) } \\
\end{array}$ & $\begin{array}{c}\text { Target } \\
\text { Density } \\
(\mathrm{g} / \mathrm{mL})\end{array}$ & $\begin{array}{c}\text { Average } \\
\text { Jet }^{(\mathbf{b})} \\
\text { Velocity }^{(\mathbf{b})} \\
\text { (ft/s) } \\
\end{array}$ & $\begin{array}{c}\text { Actual } \\
\text { Dynamic } \\
\text { Viscosity } \\
\text { (cP) } \\
\end{array}$ & $\begin{array}{c}\text { Average } \\
\text { Capture } \\
\text { Velocity }^{(b)} \\
\text { (ft/s) } \\
\end{array}$ & $\begin{array}{l}\text { Actual } \\
\text { Density } \\
(\mathrm{g} / \mathrm{mL})\end{array}$ & $\begin{array}{c}\text { Actual } \\
\text { Kinematic } \\
\text { Viscosity } \\
\left(\mathbf{m}^{2} / \mathbf{s}\right) \\
\end{array}$ \\
\hline 1 & 120 & $\mathrm{H}$ & 28.72 & 3.3 & 7.3 & 1.284 & 29.17 & 2.600 & 7.132 & 1.300 & 2.000 \\
\hline 2 & 120 & $\mathrm{H}$ & 33.51 & 3.3 & 7.3 & 1.284 & 33.58 & 2.300 & 7.120 & 1.220 & 1.885 \\
\hline 3 & 120 & $\mathrm{~T}$ & 31.12 & 3.3 & 7.3 & 1.284 & 31.28 & 3.400 & 7.183 & 1.315 & 2.586 \\
\hline 4 & 120 & $\mathrm{~T}$ & 38.30 & 3.3 & 7.3 & 1.284 & 41.17 & 3.200 & 7.224 & 1.310 & 2.443 \\
\hline 5 & 120 & $\mathrm{~T}$ & 38.30 & 3.3 & 7.3 & 1.284 & 38.41 & 2.300 & 7.165 & 1.295 & 1.776 \\
\hline 6 & 120 & $\mathrm{H}$ & 31.12 & 8 & 3.8 & 1.320 & 30.86 & 8.100 & 3.701 & 1.310 & 6.183 \\
\hline 7 & 120 & $\mathrm{H}$ & 31.12 & 8 & 3.8 & 1.320 & 31.68 & 6.900 & 3.752 & 1.305 & 5.287 \\
\hline 8 & 120 & $\mathrm{H}$ & 35.90 & 8 & 3.8 & 1.320 & 36.57 & 8.300 & 3.738 & 1.315 & 6.312 \\
\hline 9 & 120 & $\mathrm{H}$ & 28.72 & 8 & 7.3 & 1.320 & 29.15 & 8.600 & 7.202 & 1.315 & 6.540 \\
\hline 10 & 120 & $\mathrm{H}$ & 38.30 & 8 & 7.3 & 1.320 & 38.44 & 7.000 & 7.140 & 1.310 & 5.344 \\
\hline 11 & 120 & $\mathrm{H}$ & 33.51 & 8 & 11.3 & 1.320 & 33.68 & 8.500 & 11.117 & 1.305 & 6.513 \\
\hline 12 & 120 & $\mathrm{H}$ & 33.51 & 8 & 11.3 & 1.320 & 33.92 & 8.700 & 11.059 & 1.320 & 6.591 \\
\hline 13 & 120 & $\mathrm{~T}$ & 33.51 & 8 & 3.8 & 1.320 & 33.20 & 7.800 & 3.731 & 1.315 & 5.932 \\
\hline 14 & 120 & $\mathrm{~T}$ & 28.72 & 8 & 7.3 & 1.320 & 28.96 & 8.000 & 7.191 & 1.320 & 6.061 \\
\hline 15 & 120 & $\mathrm{~T}$ & 38.30 & 8 & 7.3 & 1.320 & 38.55 & 7.800 & 7.198 & 1.340 & 5.821 \\
\hline 16 & 120 & $\mathrm{~T}$ & 33.51 & 8 & 11.3 & 1.320 & 34.03 & 8.300 & 11.204 & 1.315 & 6.312 \\
\hline 17 & 120 & $\mathrm{~T}$ & 33.51 & 8 & 11.3 & 1.320 & 33.35 & 6.800 & 11.128 & 1.310 & 5.191 \\
\hline 18 & 120 & $\mathrm{H}$ & 28.72 & 14.6 & 11.3 & 1.370 & 28.59 & 16.500 & 11.161 & 1.355 & 12.177 \\
\hline 19 & 120 & $\mathrm{H}$ & 33.51 & 14.6 & 11.3 & 1.370 & 34.70 & 17.100 & 11.110 & 1.355 & 12.620 \\
\hline 20 & 120 & $\mathrm{H}$ & 38.30 & 14.6 & 11.3 & 1.370 & 39.35 & 13.500 & 11.050 & 1.355 & 9.963 \\
\hline 21 & 120 & $\mathrm{H}$ & 31.12 & 14.6 & 11.3 & 1.370 & 31.03 & 13.900 & 11.168 & 1.355 & 10.258 \\
\hline 22 & 120 & $\mathrm{~T}$ & 35.90 & 14.6 & 11.3 & 1.370 & 35.65 & 15.100 & 11.061 & 1.350 & 11.185 \\
\hline 23 & 120 & $\mathrm{~T}$ & 31.12 & 3.3 & 3.8 & 1.284 & 31.05 & 3.300 & 3.746 & 1.335 & 2.472 \\
\hline 24 & 120 & $\mathrm{~T}$ & 33.51 & 3.3 & 3.8 & 1.284 & 33.74 & 3.200 & 3.753 & 1.335 & 2.397 \\
\hline 25 & 120 & $\mathrm{~T}$ & 35.90 & 3.3 & 3.8 & 1.284 & 35.97 & 3.300 & 3.743 & 1.350 & 2.444 \\
\hline 26 & 120 & $\mathrm{H}$ & 33.51 & 3.3 & 3.8 & 1.284 & 35.00 & 3.200 & 3.762 & 1.340 & 2.388 \\
\hline
\end{tabular}

(a) $B S=$ Base Simulant: $\mathrm{H}=$ High, $\mathrm{T}=$ Typical.

(b) The average jet velocity and capture velocity are taken over the five transfer batches, because the pre-transfer samples were taken over a much shorter time frame and results were not as accurate. Specifically, the averages come from measurements taken every second, but the start and stop times for those data points were known only to the closest minute. Pre-transfer collection times ranged from $25-55$ seconds for the 43-inch tank and 1-2 minutes for the 120-inch tank. 
Table A.2. Concentrations of Gibbsite, Stainless Steel, Sand, and $\mathrm{ZrO}_{2}$ in Pre-Transfer and Five BatchTransfer Samples for 26 Mixing and Transfer Performance Tests Performed in 43-inch and 120-inch Tanks

\begin{tabular}{|c|c|c|c|c|c|c|}
\hline \multirow[b]{2}{*}{$\begin{array}{c}\text { Test } \\
\text { Number }\end{array}$} & \multirow[b]{2}{*}{$\begin{array}{l}\text { Tank Size } \\
\quad \text { (in.) }\end{array}$} & \multirow[b]{2}{*}{ Batch $^{(a)}$} & \multicolumn{4}{|c|}{ Concentration (lb/gal slurry) } \\
\hline & & & Gibbsite & $\begin{array}{c}\text { Stainless } \\
\text { Steel }\end{array}$ & Sand & $\mathrm{ZrO}_{2}$ \\
\hline 1 & 43 & 0 & 0.04317 & 0.00921 & 0.59358 & 0.08570 \\
\hline 1 & 43 & 1 & 0.05072 & 0.00400 & 0.60486 & 0.09664 \\
\hline 1 & 43 & 2 & 0.04992 & 0.00506 & 0.57318 & 0.09373 \\
\hline 1 & 43 & 3 & 0.04930 & 0.00664 & 0.54771 & 0.09485 \\
\hline 1 & 43 & 4 & 0.04743 & 0.00674 & 0.51225 & 0.08888 \\
\hline 1 & 43 & 5 & 0.03924 & 0.00607 & 0.48287 & 0.07979 \\
\hline 2 & 43 & 0 & 0.03661 & 0.42938 & 0.85051 & 0.08340 \\
\hline 2 & 43 & 1 & 0.03910 & 0.34921 & 0.85281 & 0.09669 \\
\hline 2 & 43 & 2 & 0.03871 & 0.32766 & 0.76145 & 0.09173 \\
\hline 2 & 43 & 3 & 0.03409 & 0.27006 & 0.56420 & 0.07319 \\
\hline 2 & 43 & 4 & 0.04028 & 0.28151 & 0.57824 & 0.09029 \\
\hline 2 & 43 & 5 & 0.04145 & 0.30448 & 0.64408 & 0.09302 \\
\hline 3 & 43 & 0 & 0.87934 & 0.02626 & 0.13679 & 0.13068 \\
\hline 3 & 43 & 1 & 1.07684 & 0.01772 & 0.15665 & 0.14838 \\
\hline 3 & 43 & 2 & 1.08232 & 0.01567 & 0.14861 & 0.15124 \\
\hline 3 & 43 & 3 & 1.08549 & 0.01641 & 0.16258 & 0.14935 \\
\hline 3 & 43 & 4 & 1.05189 & 0.01162 & 0.14287 & 0.14358 \\
\hline 3 & 43 & 5 & 1.01899 & 0.01365 & 0.13433 & 0.14043 \\
\hline 4 & 43 & 0 & 0.94665 & 0.02994 & 0.22383 & 0.13330 \\
\hline 4 & 43 & 1 & 1.04425 & 0.03219 & 0.18879 & 0.14346 \\
\hline 4 & 43 & 2 & 1.03941 & 0.03586 & 0.20152 & 0.14344 \\
\hline 4 & 43 & 3 & 1.06132 & 0.03312 & 0.20258 & 0.14536 \\
\hline 4 & 43 & 4 & 1.04478 & 0.03604 & 0.18462 & 0.14378 \\
\hline 4 & 43 & 5 & 1.04872 & 0.03258 & 0.18435 & 0.14320 \\
\hline 5 & 43 & 0 & 1.06458 & 0.03757 & 0.16748 & 0.15292 \\
\hline 5 & 43 & 1 & 1.08866 & 0.03757 & 0.19764 & 0.15503 \\
\hline 5 & 43 & 2 & 1.06967 & 0.03369 & 0.19946 & 0.15345 \\
\hline 5 & 43 & 3 & 1.04120 & 0.03619 & 0.19750 & 0.15184 \\
\hline 5 & 43 & 4 & 1.00823 & 0.03333 & 0.19134 & 0.14279 \\
\hline 5 & 43 & 5 & 1.05830 & 0.03390 & 0.18204 & 0.15320 \\
\hline 6 & 43 & 0 & 0.04902 & 0.07330 & 0.87722 & 0.11534 \\
\hline 6 & 43 & 1 & 0.04982 & 0.05498 & 0.79263 & 0.10814 \\
\hline 6 & 43 & 2 & 0.03751 & 0.05132 & 0.70386 & 0.09305 \\
\hline 6 & 43 & 3 & 0.04435 & 0.04422 & 0.69812 & 0.10043 \\
\hline 6 & 43 & 4 & 0.04363 & 0.04630 & 0.68176 & 0.09663 \\
\hline 6 & 43 & 5 & 0.04159 & 0.05660 & 0.74480 & 0.09917 \\
\hline 7 & 43 & 0 & 0.04584 & 0.04705 & 0.73401 & 0.11510 \\
\hline 7 & 43 & 1 & 0.02888 & 0.04682 & 0.51581 & 0.07591 \\
\hline 7 & 43 & 2 & 0.03228 & 0.03847 & 0.56363 & 0.08395 \\
\hline 7 & 43 & 3 & 0.02731 & 0.02828 & 0.45251 & 0.07171 \\
\hline 7 & 43 & 4 & 0.03208 & 0.02412 & 0.48622 & 0.07736 \\
\hline 7 & 43 & 5 & 0.03148 & 0.03477 & 0.49752 & 0.07767 \\
\hline
\end{tabular}


Table A.2. Concentrations of Gibbsite, Stainless Steel, Sand, and $\mathrm{ZrO}_{2}$ in Pre-Transfer and Five Batch-Transfer Samples for 26 Mixing and Transfer Performance Tests Performed in 43-inch and 120-inch Tanks (cont.)

\begin{tabular}{|c|c|c|c|c|c|c|}
\hline \multirow[b]{2}{*}{$\begin{array}{c}\text { Test } \\
\text { Number }\end{array}$} & \multirow[b]{2}{*}{$\begin{array}{c}\text { Tank Size } \\
\text { (in.) }\end{array}$} & \multirow[b]{2}{*}{ Batch $^{(\mathbf{a})}$} & \multicolumn{4}{|c|}{ Concentration (lb/gal slurry) } \\
\hline & & & Gibbsite & $\begin{array}{c}\text { Stainless } \\
\text { Steel }\end{array}$ & Sand & $\mathrm{ZrO}_{2}$ \\
\hline 8 & 43 & 0 & 0.03170 & 0.32162 & 0.71011 & 0.07228 \\
\hline 8 & 43 & 1 & 0.03582 & 0.34683 & 0.82394 & 0.07456 \\
\hline 8 & 43 & 2 & 0.03332 & 0.34408 & 0.74868 & 0.08204 \\
\hline 8 & 43 & 3 & 0.03427 & 0.35855 & 0.76798 & 0.07802 \\
\hline 8 & 43 & 4 & 0.03739 & 0.35795 & 0.77762 & 0.07974 \\
\hline 8 & 43 & 5 & 0.04082 & 0.36281 & 0.77471 & 0.08284 \\
\hline 9 & 43 & 0 & 0.04474 & 0.01903 & 0.59294 & 0.11374 \\
\hline 9 & 43 & 1 & 0.04429 & 0.01704 & 0.57118 & 0.10901 \\
\hline 9 & 43 & 2 & 0.04856 & 0.01023 & 0.57086 & 0.11176 \\
\hline 9 & 43 & 3 & 0.04421 & 0.00931 & 0.55424 & 0.09575 \\
\hline 9 & 43 & 4 & 0.04376 & 0.00738 & 0.53886 & 0.09342 \\
\hline 9 & 43 & 5 & 0.04278 & 0.01111 & 0.58076 & 0.09702 \\
\hline 10 & 43 & 0 & 0.03768 & 0.30545 & 0.92578 & 0.09460 \\
\hline 10 & 43 & 1 & 0.04813 & 0.31825 & 0.99288 & 0.12351 \\
\hline 10 & 43 & 2 & 0.04942 & 0.31324 & 0.85971 & 0.12360 \\
\hline 10 & 43 & 3 & 0.04204 & 0.30292 & 0.84410 & 0.11473 \\
\hline 10 & 43 & 4 & 0.03973 & 0.30170 & 0.80667 & 0.10910 \\
\hline 10 & 43 & 5 & 0.04267 & 0.25109 & 0.79057 & 0.10354 \\
\hline 11 & 43 & 0 & 0.04740 & 0.10406 & 0.82084 & 0.12284 \\
\hline 11 & 43 & 1 & 0.04620 & 0.12549 & 0.69693 & 0.10434 \\
\hline 11 & 43 & 2 & 0.04672 & 0.11156 & 0.69399 & 0.10749 \\
\hline 11 & 43 & 3 & 0.04262 & 0.11160 & 0.72148 & 0.10306 \\
\hline 11 & 43 & 4 & 0.03888 & 0.09591 & 0.70863 & 0.09853 \\
\hline 11 & 43 & 5 & 0.04159 & 0.08218 & 0.71119 & 0.10729 \\
\hline 12 & 43 & 0 & 0.04321 & 0.10538 & 0.90160 & 0.09964 \\
\hline 12 & 43 & 1 & 0.04255 & 0.12755 & 0.82709 & 0.10430 \\
\hline 12 & 43 & 2 & 0.04694 & 0.12192 & 0.81354 & 0.11308 \\
\hline 12 & 43 & 3 & 0.04724 & 0.12782 & 0.77863 & 0.10787 \\
\hline 12 & 43 & 4 & 0.04367 & 0.14744 & 0.79903 & 0.10196 \\
\hline 12 & 43 & 5 & 0.04557 & 0.14991 & 0.79920 & 0.10828 \\
\hline 13 & 43 & 0 & 1.01400 & 0.02048 & 0.22612 & 0.13354 \\
\hline 13 & 43 & 1 & 0.94452 & 0.01735 & 0.22610 & 0.13850 \\
\hline 13 & 43 & 2 & 1.00386 & 0.01453 & 0.24224 & 0.13992 \\
\hline 13 & 43 & 3 & 1.02786 & 0.01352 & 0.23745 & 0.14055 \\
\hline 13 & 43 & 4 & 1.05965 & 0.00981 & 0.21848 & 0.14417 \\
\hline 13 & 43 & 5 & 1.03107 & 0.00862 & 0.21374 & 0.14141 \\
\hline 14 & 43 & 0 & 0.73647 & 0.00173 & 0.09857 & 0.10047 \\
\hline 14 & 43 & 1 & 0.96024 & 0.00098 & 0.11059 & 0.13318 \\
\hline 14 & 43 & 2 & 1.02836 & 0.00078 & 0.10701 & 0.13579 \\
\hline 14 & 43 & 3 & 0.83776 & 0.00069 & 0.09472 & 0.11804 \\
\hline 14 & 43 & 4 & 1.07583 & 0.00053 & 0.10318 & 0.13343 \\
\hline 14 & 43 & 5 & 0.99388 & 0.00053 & 0.10788 & 0.12409 \\
\hline 15 & 43 & 0 & 0.99492 & 0.05135 & 0.15908 & 0.14094 \\
\hline 15 & 43 & 1 & 1.14839 & 0.06433 & 0.22192 & 0.16384 \\
\hline
\end{tabular}


Table A.2. Concentrations of Gibbsite, Stainless Steel, Sand, and $\mathrm{ZrO}_{2}$ in Pre-Transfer and Five Batch-Transfer Samples for 26 Mixing and Transfer Performance Tests Performed in 43-inch and 120-inch Tanks (cont.)

\begin{tabular}{|c|c|c|c|c|c|c|}
\hline \multirow[b]{2}{*}{$\begin{array}{c}\text { Test } \\
\text { Number }\end{array}$} & \multirow[b]{2}{*}{$\begin{array}{l}\text { Tank Size } \\
\text { (in.) }\end{array}$} & \multirow[b]{2}{*}{ Batch $^{(a)}$} & \multicolumn{4}{|c|}{ Concentration (lb/gal slurry) } \\
\hline & & & Gibbsite & $\begin{array}{c}\text { Stainless } \\
\text { Steel }\end{array}$ & Sand & $\mathrm{ZrO}_{2}$ \\
\hline 15 & 43 & 2 & 1.08900 & 0.05772 & 0.20990 & 0.14839 \\
\hline 15 & 43 & 3 & 1.07118 & 0.05325 & 0.19780 & 0.14738 \\
\hline 15 & 43 & 4 & 1.04611 & 0.04851 & 0.17418 & 0.14215 \\
\hline 15 & 43 & 5 & 1.20166 & 0.05719 & 0.19861 & 0.16564 \\
\hline 16 & 43 & 0 & 1.08890 & 0.05519 & 0.19541 & 0.15215 \\
\hline 16 & 43 & 1 & 1.13259 & 0.03993 & 0.20127 & 0.14884 \\
\hline 16 & 43 & 2 & 1.14131 & 0.03380 & 0.22149 & 0.15358 \\
\hline 16 & 43 & 3 & 1.17134 & 0.03329 & 0.24183 & 0.16341 \\
\hline 16 & 43 & 4 & 1.13739 & 0.02562 & 0.20116 & 0.15250 \\
\hline 16 & 43 & 5 & 1.16034 & 0.02414 & 0.23347 & 0.15928 \\
\hline 17 & 43 & 0 & 1.01540 & 0.03204 & 0.17448 & 0.14444 \\
\hline 17 & 43 & 1 & 1.01136 & 0.00880 & 0.16597 & 0.13502 \\
\hline 17 & 43 & 2 & 1.04247 & 0.01015 & 0.17525 & 0.13826 \\
\hline 17 & 43 & 3 & 1.02722 & 0.01222 & 0.18511 & 0.13817 \\
\hline 17 & 43 & 4 & 1.06557 & 0.01593 & 0.17555 & 0.14510 \\
\hline 17 & 43 & 5 & 1.11005 & 0.02032 & 0.16453 & 0.15480 \\
\hline 18 & 43 & 0 & 0.03276 & 0.02648 & 0.35978 & 0.09045 \\
\hline 18 & 43 & 1 & 0.04633 & 0.00732 & 0.56921 & 0.11031 \\
\hline 18 & 43 & 2 & 0.04657 & 0.00925 & 0.54700 & 0.11833 \\
\hline 18 & 43 & 3 & 0.03679 & 0.01340 & 0.43810 & 0.09887 \\
\hline 18 & 43 & 4 & 0.04689 & 0.01647 & 0.56046 & 0.12738 \\
\hline 18 & 43 & 5 & 0.04805 & 0.03600 & 0.57786 & 0.13245 \\
\hline 19 & 43 & 0 & 0.04873 & 0.14452 & 0.79409 & 0.12470 \\
\hline 19 & 43 & 1 & 0.04263 & 0.14137 & 0.76584 & 0.10732 \\
\hline 19 & 43 & 2 & 0.04380 & 0.12654 & 0.73706 & 0.10758 \\
\hline 19 & 43 & 3 & 0.05364 & 0.13619 & 0.84778 & 0.12168 \\
\hline 19 & 43 & 4 & 0.03954 & 0.11102 & 0.72883 & 0.10282 \\
\hline 19 & 43 & 5 & 0.04270 & 0.09202 & 0.77207 & 0.09729 \\
\hline 20 & 43 & 0 & 0.04576 & 0.33293 & 0.91522 & 0.11951 \\
\hline 20 & 43 & 1 & 0.04137 & 0.28197 & 0.90268 & 0.10622 \\
\hline 20 & 43 & 2 & 0.03836 & 0.26821 & 0.83614 & 0.10516 \\
\hline 20 & 43 & 3 & 0.04241 & 0.21773 & 0.80069 & 0.11951 \\
\hline 20 & 43 & 4 & 0.04212 & 0.16693 & 0.83681 & 0.10764 \\
\hline 20 & 43 & 5 & 0.04387 & 0.15311 & 0.88430 & 0.10899 \\
\hline 21 & 43 & 0 & 0.04785 & 0.04834 & 0.76279 & 0.11667 \\
\hline 21 & 43 & 1 & 0.04513 & 0.02983 & 0.74189 & 0.11738 \\
\hline 21 & 43 & 2 & 0.04432 & 0.02174 & 0.71791 & 0.10879 \\
\hline 21 & 43 & 3 & 0.04349 & 0.01724 & 0.69352 & 0.09847 \\
\hline 21 & 43 & 4 & 0.04446 & 0.02096 & 0.73949 & 0.10691 \\
\hline 21 & 43 & 5 & 0.04589 & 0.01496 & 0.73810 & 0.10706 \\
\hline 22 & 43 & 0 & 1.12355 & 0.02414 & 0.21511 & 0.14919 \\
\hline 22 & 43 & 1 & 1.09950 & 0.00073 & 0.17915 & 0.16796 \\
\hline 22 & 43 & 2 & 0.81726 & 0.01317 & 0.18599 & 0.12439 \\
\hline 22 & 43 & 3 & 1.33338 & 0.01225 & 0.20187 & 0.16805 \\
\hline
\end{tabular}


Table A.2. Concentrations of Gibbsite, Stainless Steel, Sand, and $\mathrm{ZrO}_{2}$ in Pre-Transfer and Five Batch-Transfer Samples for 26 Mixing and Transfer Performance Tests Performed in 43-inch and 120-inch Tanks (cont.)

\begin{tabular}{|c|c|c|c|c|c|c|}
\hline \multirow[b]{2}{*}{$\begin{array}{c}\text { Test } \\
\text { Number }\end{array}$} & \multirow[b]{2}{*}{$\begin{array}{l}\text { Tank Size } \\
\quad \text { (in.) }\end{array}$} & \multirow[b]{2}{*}{$\operatorname{Batch}^{(\mathbf{a})}$} & \multicolumn{4}{|c|}{ Concentration (lb/gal slurry) } \\
\hline & & & Gibbsite & $\begin{array}{c}\text { Stainless } \\
\text { Steel }\end{array}$ & Sand & $\mathrm{ZrO}_{2}$ \\
\hline 22 & 43 & 4 & 1.12683 & 0.01322 & 0.21021 & 0.15811 \\
\hline 22 & 43 & 5 & 1.24052 & 0.00998 & 0.23250 & 0.16438 \\
\hline 23 & 43 & 0 & 1.11865 & 0.01908 & 0.12819 & 0.15139 \\
\hline 23 & 43 & 1 & 1.08676 & 0.01077 & 0.15150 & 0.14652 \\
\hline 23 & 43 & 2 & 1.08048 & 0.00891 & 0.15202 & 0.14578 \\
\hline 23 & 43 & 3 & 1.09402 & 0.00819 & 0.15415 & 0.14676 \\
\hline 23 & 43 & 4 & 1.06157 & 0.00657 & 0.14821 & 0.14156 \\
\hline 23 & 43 & 5 & 1.03770 & 0.00586 & 0.17492 & 0.13870 \\
\hline 24 & 43 & 0 & 1.10271 & 0.04112 & 0.15753 & 0.15469 \\
\hline 24 & 43 & 1 & 1.07193 & 0.02578 & 0.17266 & 0.15099 \\
\hline 24 & 43 & 2 & 1.08412 & 0.02320 & 0.17343 & 0.15070 \\
\hline 24 & 43 & 3 & 1.04372 & 0.01990 & 0.18013 & 0.14403 \\
\hline 24 & 43 & 4 & 1.09016 & 0.01776 & 0.17461 & 0.14911 \\
\hline 24 & 43 & 5 & 1.05475 & 0.01633 & 0.14833 & 0.14409 \\
\hline 25 & 43 & 0 & 1.01811 & 0.04027 & 0.18539 & 0.14360 \\
\hline 25 & 43 & 1 & 1.19124 & 0.03902 & 0.22686 & 0.16801 \\
\hline 25 & 43 & 2 & 1.19519 & 0.03593 & 0.21208 & 0.16780 \\
\hline 25 & 43 & 3 & 1.20259 & 0.03419 & 0.22768 & 0.16760 \\
\hline 25 & 43 & 4 & 1.22366 & 0.02757 & 0.19856 & 0.17027 \\
\hline 25 & 43 & 5 & 1.23112 & 0.02508 & 0.19337 & 0.17011 \\
\hline 26 & 43 & 0 & 0.05481 & 0.10996 & 0.87104 & 0.11269 \\
\hline 26 & 43 & 1 & 0.05741 & 0.09059 & 0.80330 & 0.11652 \\
\hline 26 & 43 & 2 & 0.04908 & 0.09500 & 0.74645 & 0.10591 \\
\hline 26 & 43 & 3 & 0.05287 & 0.08968 & 0.73501 & 0.10837 \\
\hline 26 & 43 & 4 & 0.05240 & 0.09114 & 0.67747 & 0.10615 \\
\hline 26 & 43 & 5 & 0.05324 & 0.09272 & 0.73494 & 0.10867 \\
\hline 1 & 120 & 0 & 0.05160 & 0.06944 & 0.77002 & 0.11591 \\
\hline 1 & 120 & 1 & 0.04657 & 0.04929 & 0.63332 & 0.09938 \\
\hline 1 & 120 & 2 & 0.04909 & 0.04770 & 0.64804 & 0.10910 \\
\hline 1 & 120 & 3 & 0.04806 & 0.07209 & 0.63207 & 0.10855 \\
\hline 1 & 120 & 4 & 0.05059 & 0.08548 & 0.67671 & 0.11033 \\
\hline 1 & 120 & 5 & 0.05091 & 0.08371 & 0.86685 & 0.12459 \\
\hline 2 & 120 & 0 & 0.04508 & 0.47022 & 0.95665 & 0.11074 \\
\hline 2 & 120 & 1 & 0.04787 & 0.49651 & 0.88722 & 0.11471 \\
\hline 2 & 120 & 2 & 0.04040 & 0.37864 & 0.67306 & 0.09098 \\
\hline 2 & 120 & 3 & 0.04153 & 0.35909 & 0.60746 & 0.10238 \\
\hline 2 & 120 & 4 & 0.04523 & 0.42466 & 0.68452 & 0.11076 \\
\hline 2 & 120 & 5 & 0.04283 & 0.35966 & 0.63824 & 0.10893 \\
\hline 3 & 120 & 0 & 1.24563 & 0.04277 & 0.22663 & 0.16018 \\
\hline 3 & 120 & 1 & 1.26000 & 0.04269 & 0.21658 & 0.16548 \\
\hline 3 & 120 & 2 & 1.22953 & 0.03817 & 0.21069 & 0.15900 \\
\hline 3 & 120 & 3 & 1.19538 & 0.03204 & 0.14668 & 0.15373 \\
\hline 3 & 120 & 4 & 1.16685 & 0.03168 & 0.15400 & 0.15161 \\
\hline 3 & 120 & 5 & 1.16158 & 0.02296 & 0.17281 & 0.15030 \\
\hline
\end{tabular}


Table A.2. Concentrations of Gibbsite, Stainless Steel, Sand, and $\mathrm{ZrO}_{2}$ in Pre-Transfer and Five Batch-Transfer Samples for 26 Mixing and Transfer Performance Tests Performed in 43-inch and 120-inch Tanks (cont.)

\begin{tabular}{|c|c|c|c|c|c|c|}
\hline \multirow[b]{2}{*}{$\begin{array}{c}\text { Test } \\
\text { Number }\end{array}$} & \multirow[b]{2}{*}{$\begin{array}{c}\text { Tank Size } \\
\text { (in.) }\end{array}$} & \multirow[b]{2}{*}{ Batch $^{(\mathbf{a})}$} & \multicolumn{4}{|c|}{ Concentration (lb/gal slurry) } \\
\hline & & & Gibbsite & $\begin{array}{c}\text { Stainless } \\
\text { Steel }\end{array}$ & Sand & $\mathrm{ZrO}_{2}$ \\
\hline 4 & 120 & 0 & 1.05923 & 0.07077 & 0.21585 & 0.14740 \\
\hline 4 & 120 & 1 & 1.17761 & 0.06116 & 0.24216 & 0.16054 \\
\hline 4 & 120 & 2 & 1.12533 & 0.04140 & 0.15961 & 0.15409 \\
\hline 4 & 120 & 3 & 1.08707 & 0.03723 & 0.17050 & 0.14955 \\
\hline 4 & 120 & 4 & 1.15714 & 0.02760 & 0.13652 & 0.15746 \\
\hline 4 & 120 & 5 & 1.08325 & 0.04649 & 0.26383 & 0.14881 \\
\hline 5 & 120 & 0 & 0.69475 & 0.06970 & 0.20931 & 0.11011 \\
\hline 5 & 120 & 1 & 1.11407 & 0.05708 & 0.21707 & 0.15400 \\
\hline 5 & 120 & 2 & 1.04437 & 0.06553 & 0.23516 & 0.14855 \\
\hline 5 & 120 & 3 & 1.02377 & 0.05501 & 0.21252 & 0.14720 \\
\hline 5 & 120 & 4 & 1.10984 & 0.05682 & 0.19940 & 0.15356 \\
\hline 5 & 120 & 5 & 1.07049 & 0.04285 & 0.19091 & 0.14943 \\
\hline 6 & 120 & 0 & 0.04251 & 0.18804 & 0.88647 & 0.10547 \\
\hline 6 & 120 & 1 & 0.04079 & 0.18865 & 0.87796 & 0.10905 \\
\hline 6 & 120 & 2 & 0.02719 & 0.14815 & 0.68989 & 0.08515 \\
\hline 6 & 120 & 3 & 0.03998 & 0.16388 & 0.73480 & 0.10605 \\
\hline 6 & 120 & 4 & 0.04259 & 0.16226 & 0.70726 & 0.10919 \\
\hline 6 & 120 & 5 & 0.04600 & 0.14174 & 0.74615 & 0.10802 \\
\hline 7 & 120 & 0 & 0.04473 & 0.19134 & 0.92373 & 0.10069 \\
\hline 7 & 120 & 1 & 0.03953 & 0.17519 & 0.80263 & 0.10183 \\
\hline 7 & 120 & 2 & 0.03617 & 0.15398 & 0.63456 & 0.08775 \\
\hline 7 & 120 & 3 & 0.03146 & 0.14283 & 0.54434 & 0.08158 \\
\hline 7 & 120 & 4 & 0.02926 & 0.15189 & 0.48157 & 0.07361 \\
\hline 7 & 120 & 5 & 0.02369 & 0.09434 & 0.42036 & 0.05920 \\
\hline 8 & 120 & 0 & 0.04673 & 0.33650 & 0.85350 & 0.11627 \\
\hline 8 & 120 & 1 & 0.02620 & 0.19481 & 0.54123 & 0.06022 \\
\hline 8 & 120 & 2 & 0.02209 & 0.13052 & 0.44112 & 0.05353 \\
\hline 8 & 120 & 3 & 0.02567 & 0.15326 & 0.42703 & 0.06638 \\
\hline 8 & 120 & 4 & 0.02500 & 0.15265 & 0.46414 & 0.06526 \\
\hline 8 & 120 & 5 & 0.02699 & 0.13259 & 0.48911 & 0.06700 \\
\hline 9 & 120 & 0 & 0.03041 & 0.21070 & 0.85598 & 0.08249 \\
\hline 9 & 120 & 1 & 0.04872 & 0.17827 & 0.83848 & 0.11020 \\
\hline 9 & 120 & 2 & 0.04804 & 0.16629 & 0.86206 & 0.10782 \\
\hline 9 & 120 & 3 & 0.05219 & 0.16724 & 0.79906 & 0.11716 \\
\hline 9 & 120 & 4 & 0.04823 & 0.14671 & 0.73547 & 0.10500 \\
\hline 9 & 120 & 5 & 0.04692 & 0.13883 & 0.76365 & 0.10743 \\
\hline 10 & 120 & 0 & 0.04580 & 0.35802 & 1.15864 & 0.11535 \\
\hline 10 & 120 & 1 & 0.03943 & 0.50948 & 0.89599 & 0.11587 \\
\hline 10 & 120 & 2 & 0.04865 & 0.38918 & 0.78614 & 0.13133 \\
\hline 10 & 120 & 3 & 0.03157 & 0.40176 & 0.75448 & 0.09222 \\
\hline 10 & 120 & 4 & 0.05960 & 0.46328 & 0.83074 & 0.14931 \\
\hline 10 & 120 & 5 & 0.04613 & 0.27207 & 0.77832 & 0.11802 \\
\hline 11 & 120 & 0 & 0.04754 & 0.33048 & 0.98302 & 0.11195 \\
\hline 11 & 120 & 1 & 0.04574 & 0.31129 & 0.89351 & 0.10538 \\
\hline
\end{tabular}


Table A.2. Concentrations of Gibbsite, Stainless Steel, Sand, and $\mathrm{ZrO}_{2}$ in Pre-Transfer and Five Batch-Transfer Samples for 26 Mixing and Transfer Performance Tests Performed in 43-inch and 120-inch Tanks (cont.)

\begin{tabular}{|c|c|c|c|c|c|c|}
\hline \multirow[b]{2}{*}{$\begin{array}{c}\text { Test } \\
\text { Number }\end{array}$} & \multirow[b]{2}{*}{$\begin{array}{l}\text { Tank Size } \\
\quad \text { (in.) }\end{array}$} & \multirow[b]{2}{*}{$\operatorname{Batch}^{(\mathbf{a})}$} & \multicolumn{4}{|c|}{ Concentration (lb/gal slurry) } \\
\hline & & & Gibbsite & $\begin{array}{l}\text { Stainless } \\
\text { Steel }\end{array}$ & Sand & $\mathrm{ZrO}_{2}$ \\
\hline 11 & 120 & 2 & 0.04801 & 0.24573 & 0.82564 & 0.11266 \\
\hline 11 & 120 & 3 & 0.05132 & 0.20164 & 0.80989 & 0.12942 \\
\hline 11 & 120 & 4 & 0.04842 & 0.16186 & 0.78763 & 0.11755 \\
\hline 11 & 120 & 5 & 0.05366 & 0.09270 & 0.81042 & 0.12448 \\
\hline 12 & 120 & 0 & 0.05135 & 0.23289 & 1.00099 & 0.12064 \\
\hline 12 & 120 & 1 & 0.04769 & 0.26887 & 0.98427 & 0.11050 \\
\hline 12 & 120 & 2 & 0.04768 & 0.27073 & 0.84065 & 0.12114 \\
\hline 12 & 120 & 3 & 0.04880 & 0.23688 & 0.81337 & 0.11855 \\
\hline 12 & 120 & 4 & 0.04859 & 0.26629 & 0.77553 & 0.11926 \\
\hline 12 & 120 & 5 & 0.05002 & 0.19020 & 0.85364 & 0.11690 \\
\hline 13 & 120 & 0 & 1.09927 & 0.05179 & 0.18120 & 0.15704 \\
\hline 13 & 120 & 1 & 1.09863 & 0.04780 & 0.19398 & 0.14798 \\
\hline 13 & 120 & 2 & 1.07920 & 0.04522 & 0.20830 & 0.15517 \\
\hline 13 & 120 & 3 & 1.09083 & 0.03957 & 0.20448 & 0.15258 \\
\hline 13 & 120 & 4 & 1.13985 & 0.03885 & 0.21882 & 0.16125 \\
\hline 13 & 120 & 5 & 1.05501 & 0.02905 & 0.19593 & 0.15176 \\
\hline 14 & 120 & 0 & 0.86995 & 0.02781 & 0.19812 & 0.13335 \\
\hline 14 & 120 & 1 & 0.87864 & 0.02324 & 0.17577 & 0.14465 \\
\hline 14 & 120 & 2 & 0.87670 & 0.02352 & 0.20689 & 0.14700 \\
\hline 14 & 120 & 3 & 1.01450 & 0.01499 & 0.20398 & 0.15503 \\
\hline 14 & 120 & 4 & 0.94155 & 0.01096 & 0.19264 & 0.14142 \\
\hline 14 & 120 & 5 & 0.83155 & 0.01026 & 0.22012 & 0.13531 \\
\hline 15 & 120 & 0 & 0.87549 & 0.05617 & 0.17645 & 0.09994 \\
\hline 15 & 120 & 1 & 1.35696 & 0.09227 & 0.26339 & 0.16422 \\
\hline 15 & 120 & 2 & 1.19512 & 0.07520 & 0.20370 & 0.13772 \\
\hline 15 & 120 & 3 & 1.05991 & 0.07062 & 0.20429 & 0.12287 \\
\hline 15 & 120 & 4 & 1.11653 & 0.07309 & 0.19777 & 0.13051 \\
\hline 15 & 120 & 5 & 0.94667 & 0.05814 & 0.16383 & 0.11142 \\
\hline 16 & 120 & 0 & 1.02463 & 0.04482 & 0.16686 & 0.14204 \\
\hline 16 & 120 & 1 & 1.06972 & 0.05159 & 0.22144 & 0.16622 \\
\hline 16 & 120 & 2 & 1.07418 & 0.04979 & 0.21220 & 0.15683 \\
\hline 16 & 120 & 3 & 1.03993 & 0.04039 & 0.20446 & 0.14976 \\
\hline 16 & 120 & 4 & 1.00932 & 0.03874 & 0.17495 & 0.14678 \\
\hline 16 & 120 & 5 & 1.06493 & 0.03525 & 0.18431 & 0.15075 \\
\hline 17 & 120 & 0 & 1.07614 & 0.05779 & 0.20929 & 0.15494 \\
\hline 17 & 120 & 1 & 1.04640 & 0.05102 & 0.19479 & 0.15253 \\
\hline 17 & 120 & 2 & 1.05226 & 0.04738 & 0.21574 & 0.15291 \\
\hline 17 & 120 & 3 & 1.06073 & 0.04729 & 0.21438 & 0.15155 \\
\hline 17 & 120 & 4 & 1.05383 & 0.03487 & 0.25378 & 0.16517 \\
\hline 17 & 120 & 5 & 1.07488 & 0.03255 & 0.20385 & 0.15225 \\
\hline 18 & 120 & 0 & 0.05207 & 0.24834 & 0.88896 & 0.13546 \\
\hline 18 & 120 & 1 & 0.04280 & 0.16074 & 0.74002 & 0.11112 \\
\hline 18 & 120 & 2 & 0.04528 & 0.12777 & 0.71553 & 0.10674 \\
\hline 18 & 120 & 3 & 0.04339 & 0.10647 & 0.70622 & 0.10780 \\
\hline
\end{tabular}


Table A.2. Concentrations of Gibbsite, Stainless Steel, Sand, and $\mathrm{ZrO}_{2}$ in Pre-Transfer and Five Batch-Transfer Samples for 26 Mixing and Transfer Performance Tests Performed in 43-inch and 120-inch Tanks (cont.)

\begin{tabular}{|c|c|c|c|c|c|c|}
\hline \multirow[b]{2}{*}{$\begin{array}{c}\text { Test } \\
\text { Number }\end{array}$} & \multirow[b]{2}{*}{$\begin{array}{c}\text { Tank Size } \\
\text { (in.) }\end{array}$} & \multirow[b]{2}{*}{ Batch $^{(a)}$} & \multicolumn{4}{|c|}{ Concentration (lb/gal slurry) } \\
\hline & & & Gibbsite & $\begin{array}{c}\text { Stainless } \\
\text { Steel }\end{array}$ & Sand & $\mathrm{ZrO}_{2}$ \\
\hline 18 & 120 & 4 & 0.04563 & 0.09357 & 0.71237 & 0.10473 \\
\hline 18 & 120 & 5 & 0.03923 & 0.10256 & 0.68589 & 0.09530 \\
\hline 19 & 120 & 0 & 0.04530 & 0.26022 & 0.95001 & 0.10767 \\
\hline 19 & 120 & 1 & 0.04304 & 0.26256 & 0.86980 & 0.11989 \\
\hline 19 & 120 & 2 & 0.04438 & 0.19856 & 0.93541 & 0.11223 \\
\hline 19 & 120 & 3 & 0.04833 & 0.19012 & 0.90576 & 0.12016 \\
\hline 19 & 120 & 4 & 0.04795 & 0.19643 & 0.88027 & 0.11406 \\
\hline 19 & 120 & 5 & 0.04522 & 0.19175 & 0.88911 & 0.10640 \\
\hline 20 & 120 & 0 & 0.04273 & 0.53969 & 1.05529 & 0.08995 \\
\hline 20 & 120 & 1 & 0.05277 & 0.37097 & 1.02780 & 0.13517 \\
\hline 20 & 120 & 2 & 0.05118 & 0.32934 & 0.94783 & 0.12140 \\
\hline 20 & 120 & 3 & 0.05173 & 0.19993 & 0.88768 & 0.11994 \\
\hline 20 & 120 & 4 & 0.04577 & 0.32739 & 0.88610 & 0.10945 \\
\hline 20 & 120 & 5 & 0.04515 & 0.24971 & 0.88831 & 0.11039 \\
\hline 21 & 120 & 0 & 0.04186 & 0.25167 & 0.87427 & 0.10949 \\
\hline 21 & 120 & 1 & 0.04686 & 0.20012 & 0.91094 & 0.11257 \\
\hline 21 & 120 & 2 & 0.04615 & 0.14151 & 0.86852 & 0.10415 \\
\hline 21 & 120 & 3 & 0.04647 & 0.12101 & 0.83398 & 0.10369 \\
\hline 21 & 120 & 4 & 0.04614 & 0.13404 & 0.82556 & 0.10316 \\
\hline 21 & 120 & 5 & 0.04705 & 0.13480 & 0.87075 & 0.11476 \\
\hline 22 & 120 & 0 & 1.10878 & 0.07108 & 0.14980 & 0.14980 \\
\hline 22 & 120 & 1 & 1.02918 & 0.05840 & 0.19983 & 0.16388 \\
\hline 22 & 120 & 2 & 1.13567 & 0.06223 & 0.22886 & 0.16790 \\
\hline 22 & 120 & 3 & 1.10911 & 0.05671 & 0.24205 & 0.16871 \\
\hline 22 & 120 & 4 & 1.14357 & 0.03989 & 0.24596 & 0.17122 \\
\hline 22 & 120 & 5 & 1.17509 & 0.03738 & 0.28253 & 0.18111 \\
\hline 23 & 120 & 0 & 0.99046 & 0.04828 & 0.21945 & 0.14220 \\
\hline 23 & 120 & 1 & 0.99953 & 0.03203 & 0.14048 & 0.14010 \\
\hline 23 & 120 & 2 & 1.01659 & 0.03199 & 0.16465 & 0.14284 \\
\hline 23 & 120 & 3 & 0.98899 & 0.02963 & 0.15913 & 0.13742 \\
\hline 23 & 120 & 4 & 0.99765 & 0.02637 & 0.15535 & 0.13793 \\
\hline 23 & 120 & 5 & 0.98590 & 0.02962 & 0.15279 & 0.13726 \\
\hline 24 & 120 & 0 & 1.05786 & 0.04385 & 0.17028 & 0.14934 \\
\hline 24 & 120 & 1 & 1.11034 & 0.03945 & 0.19626 & 0.15471 \\
\hline 24 & 120 & 2 & 1.06951 & 0.03784 & 0.18994 & 0.15179 \\
\hline 24 & 120 & 3 & 1.06614 & 0.03428 & 0.19879 & 0.14844 \\
\hline 24 & 120 & 4 & 1.08693 & 0.03278 & 0.18803 & 0.14998 \\
\hline 24 & 120 & 5 & 1.07311 & 0.03023 & 0.19401 & 0.15040 \\
\hline 25 & 120 & 0 & 1.06870 & 0.06724 & 0.17835 & 0.15004 \\
\hline 25 & 120 & 1 & 1.11181 & 0.03474 & 0.19912 & 0.15015 \\
\hline 25 & 120 & 2 & 1.04105 & 0.03890 & 0.17817 & 0.14092 \\
\hline 25 & 120 & 3 & 1.12349 & 0.04305 & 0.19383 & 0.15097 \\
\hline 25 & 120 & 4 & 1.20315 & 0.05126 & 0.20980 & 0.16183 \\
\hline 25 & 120 & 5 & 1.11014 & 0.04943 & 0.19441 & 0.15144 \\
\hline
\end{tabular}


Table A.2. Concentrations of Gibbsite, Stainless Steel, Sand, and $\mathrm{ZrO}_{2}$ in Pre-Transfer and Five Batch-Transfer Samples for 26 Mixing and Transfer Performance Tests Performed in 43-inch and 120-inch Tanks (cont.)

\begin{tabular}{ccccccc}
\hline \multirow{2}{*}{$\begin{array}{c}\text { Test } \\
\text { Number }\end{array}$} & $\begin{array}{c}\text { Tank Size } \\
\text { (in.) }\end{array}$ & Batch $^{\text {(a) }}$ & Gibbsite & $\begin{array}{c}\text { Stainless } \\
\text { Steel }\end{array}$ & Sand & ZrO $_{2}$ \\
\cline { 4 - 7 } 26 & 120 & 0 & 0.04529 & 0.09359 & 1.02126 & 0.10685 \\
26 & 120 & 1 & 0.05497 & 0.18618 & 1.02815 & 0.12090 \\
26 & 120 & 2 & 0.05111 & 0.16741 & 0.91298 & 0.11724 \\
26 & 120 & 3 & 0.05061 & 0.15288 & 0.82233 & 0.12138 \\
26 & 120 & 4 & 0.05163 & 0.17618 & 0.75385 & 0.12550 \\
26 & 120 & 5 & 0.04886 & 0.13306 & 0.80147 & 0.11848 \\
\hline
\end{tabular}

(a) Batch $=0$ corresponds to the pre-transfer sample.

(b) Concentrations were calculated as discussed in Section 3.2. 


\section{Appendix B}

Plots of Component Concentrations from Pre-Transfer Samples and Samples from Five Batch Transfers for 26 Test

Combinations Performed at Two Scales 


\section{Appendix B}

\section{Plots of Component Concentrations from Pre-Transfer Samples and Samples from Five Batch Transfers for 26 Test Combinations Performed at Two Scales}

Figure B.1 through Figure B.4 display plots of component concentrations (gibbsite, SS, sand, and $\mathrm{ZrO}_{2}$, respectively) on the $y$-axis versus pre-transfer and transfer batch numbers (1-5) on the $x$-axis. The data for all 26 test combinations in Table 2.3 and Table 2.4 are plotted, with separate plots for tests in the 43-inch and 120-inch tanks. The pre-transfer and five batch transfer values for a given test in a given tank are connected by the same color line segments to help visualize how the component concentrations change from pre-transfer through the five batch transfers. Each of the 22 distinct test combinations has a different line color (replicates for the same test conditions have the same line color). The tests that are replicate pairs also have plotting symbols (of the same colors as the line segments), which allows for visual comparison of replicate tests. The plots for the 43-inch tank (on the left) and the 120-inch tank (on the right) are on the same page for a given component. This arrangement allows for easy comparison of the traces of concentration versus pre-transfer/batch for the 26 test combinations) for the two tanks. This arrangement also allows the two plots in a figure to share one legend.

Figure B.5 through Figure B.8 are similar, except that the natural logarithms of the component concentrations are plotted on the $y$-axis. The natural logarithm transformation allows seeing the differences between traces for the Typical and High base simulants (which tend to be bunched together, especially for components that made up small proportions of either the Typical or High base simulant). The natural logarithm transformation also tends to linearize the relationship between component concentrations and pre-transfer/batch numbers.

Figure B.1 through Figure B.4 and Figure B.5 through Figure B.8 are discussed in Section 3.3.1 of the report. 

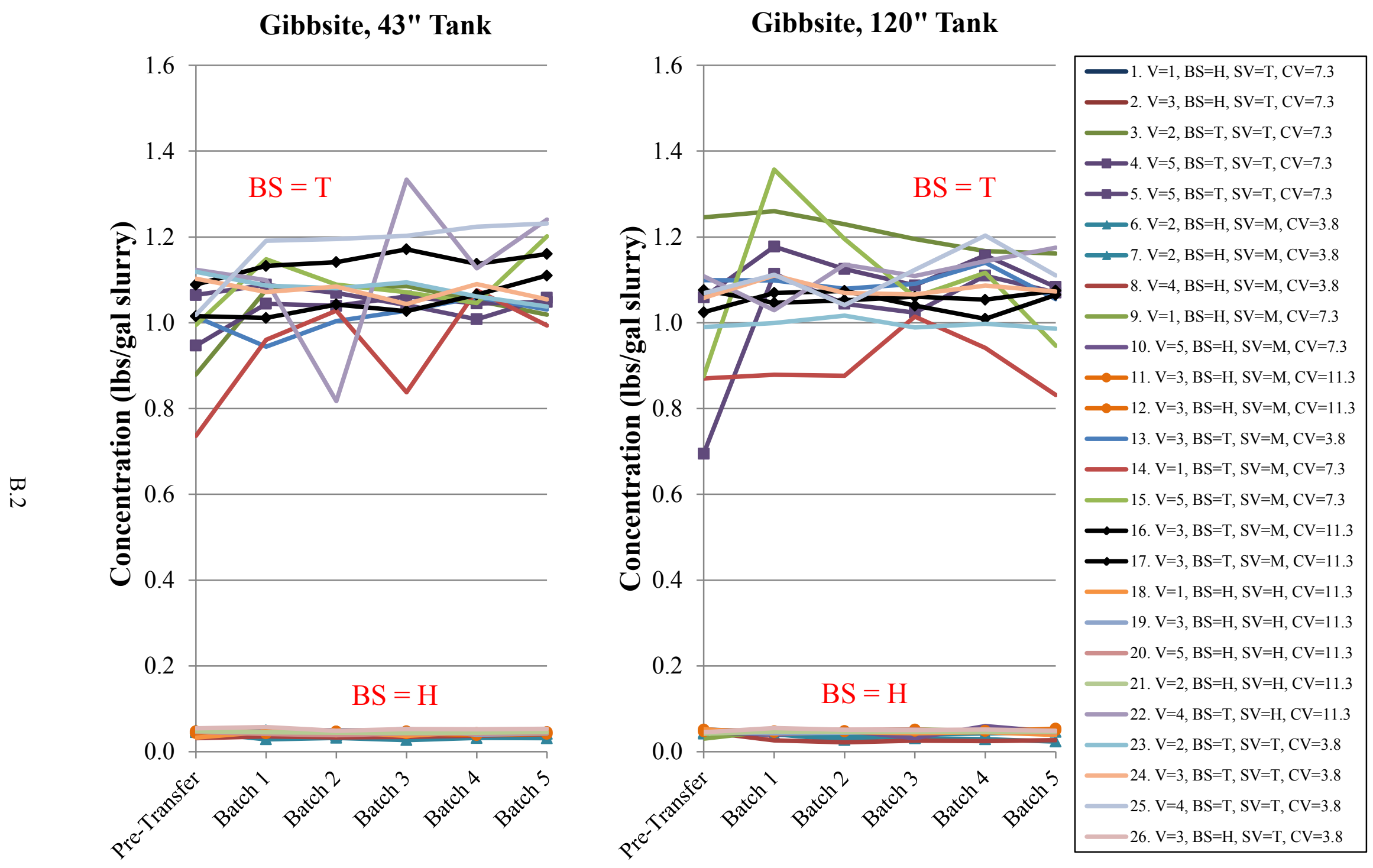

Figure B.1. Gibbsite $\left[\mathrm{Al}(\mathrm{OH})_{3}\right]$ Concentrations Plotted versus Pre-transfer and Five Batch Transfers for the 43-inch Tank and the 120-inch Tank (For Information Only) 

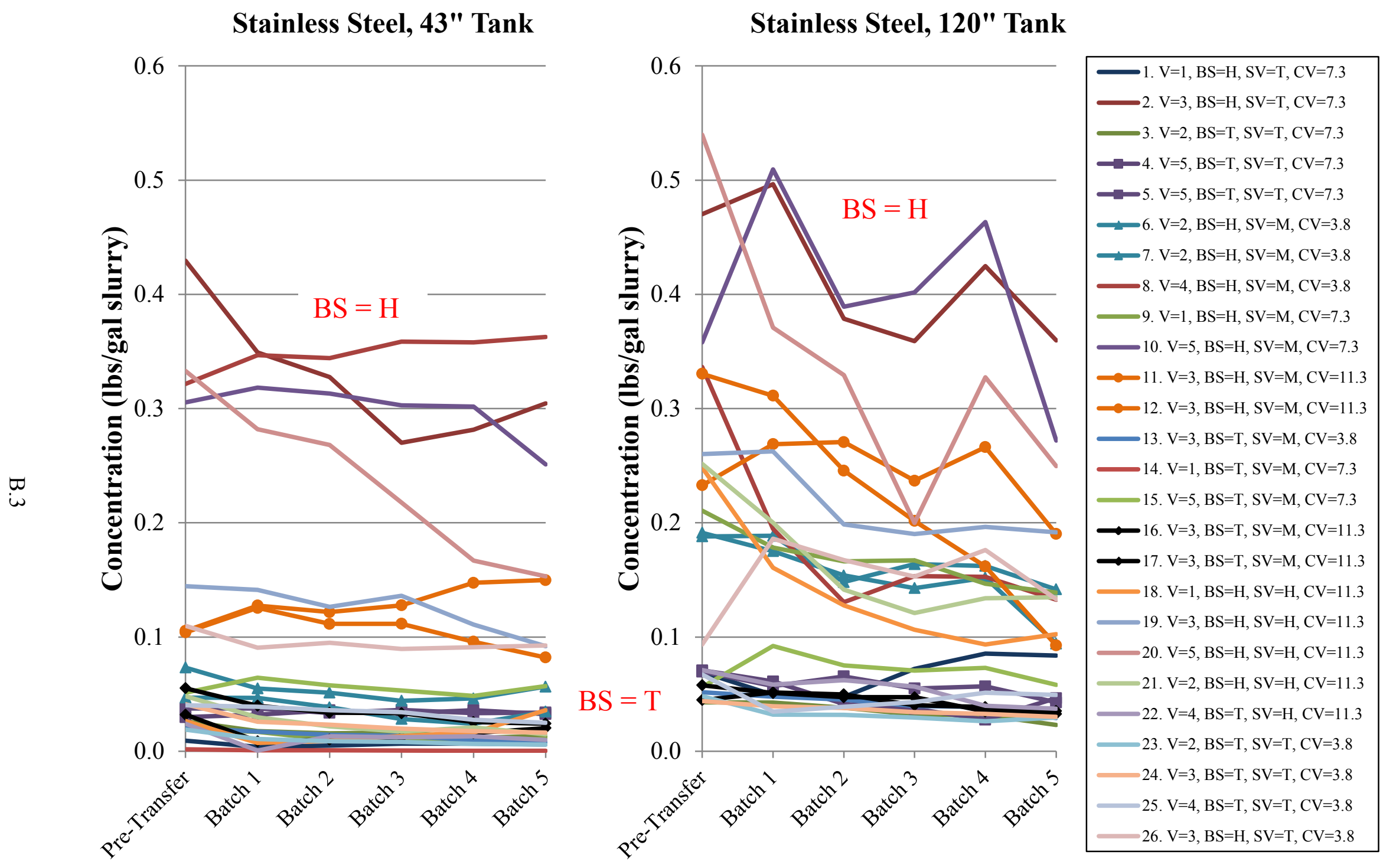

Figure B.2. Stainless Steel Concentrations Plotted versus Pre-transfer and Five Batch Transfers for the 43-inch Tank and the 120-inch Tank (For Information Only) 

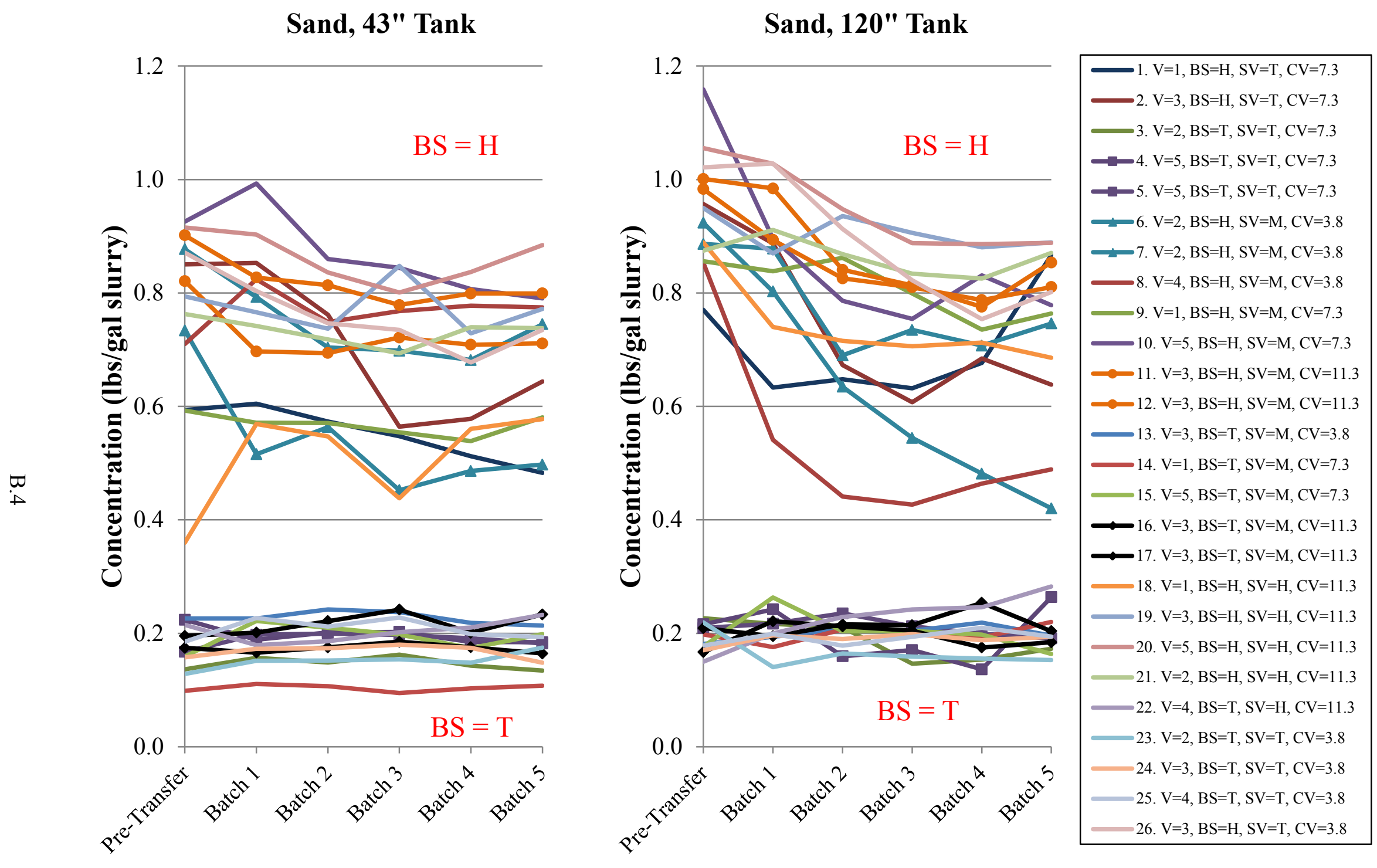

Figure B.3. Sand $\left(\mathrm{SiO}_{2}\right)$ Concentrations Plotted versus Pre-transfer and Five Batch Transfers for the 43-inch Tank and the 120-inch Tank (For Information Only) 

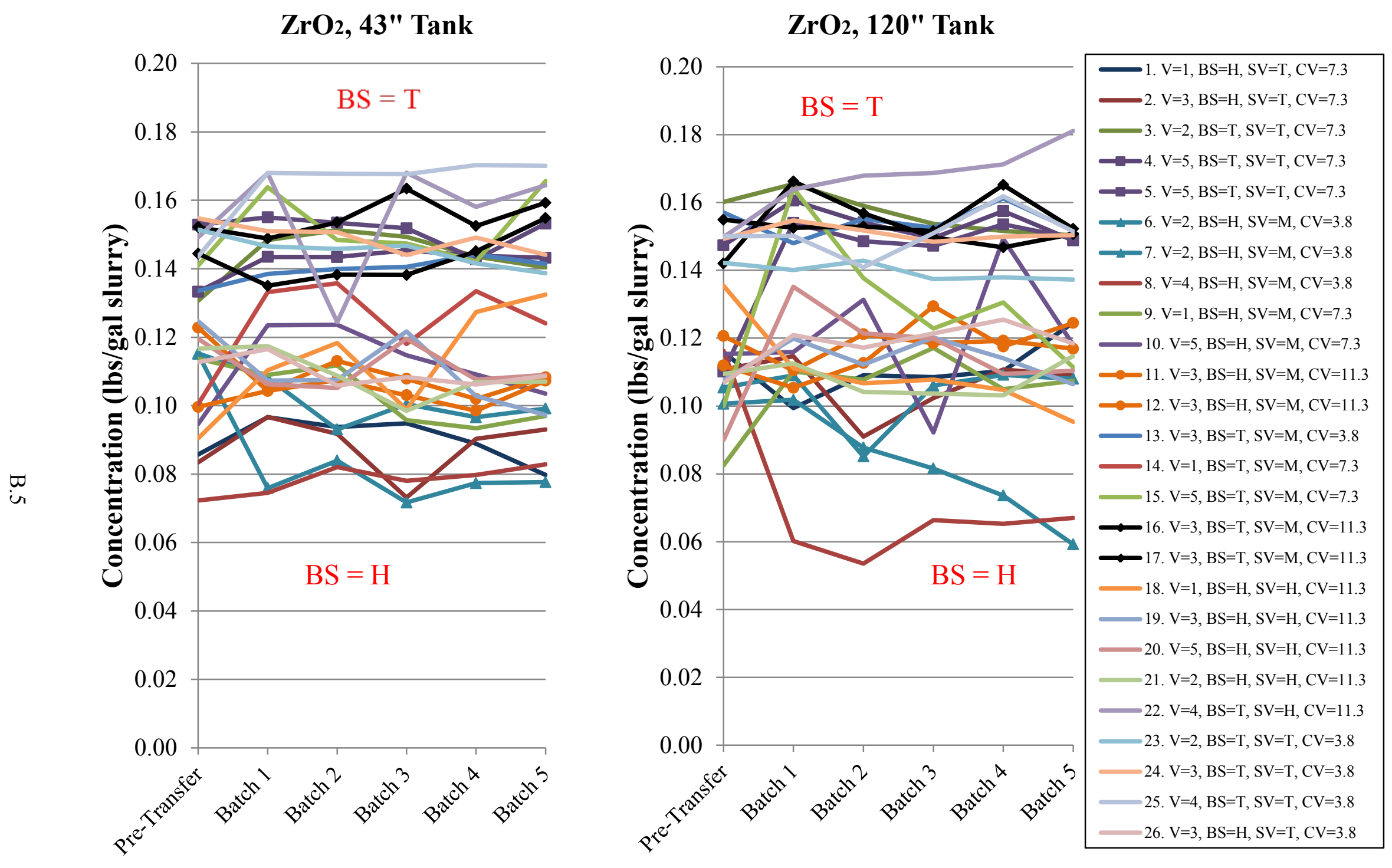

Figure B.4. $\mathrm{ZrO}_{2}$ Concentrations Plotted versus Pre-transfer and Five Batch Transfers for the 43-inch Tank and the 120-inch Tank (For Information Only) 

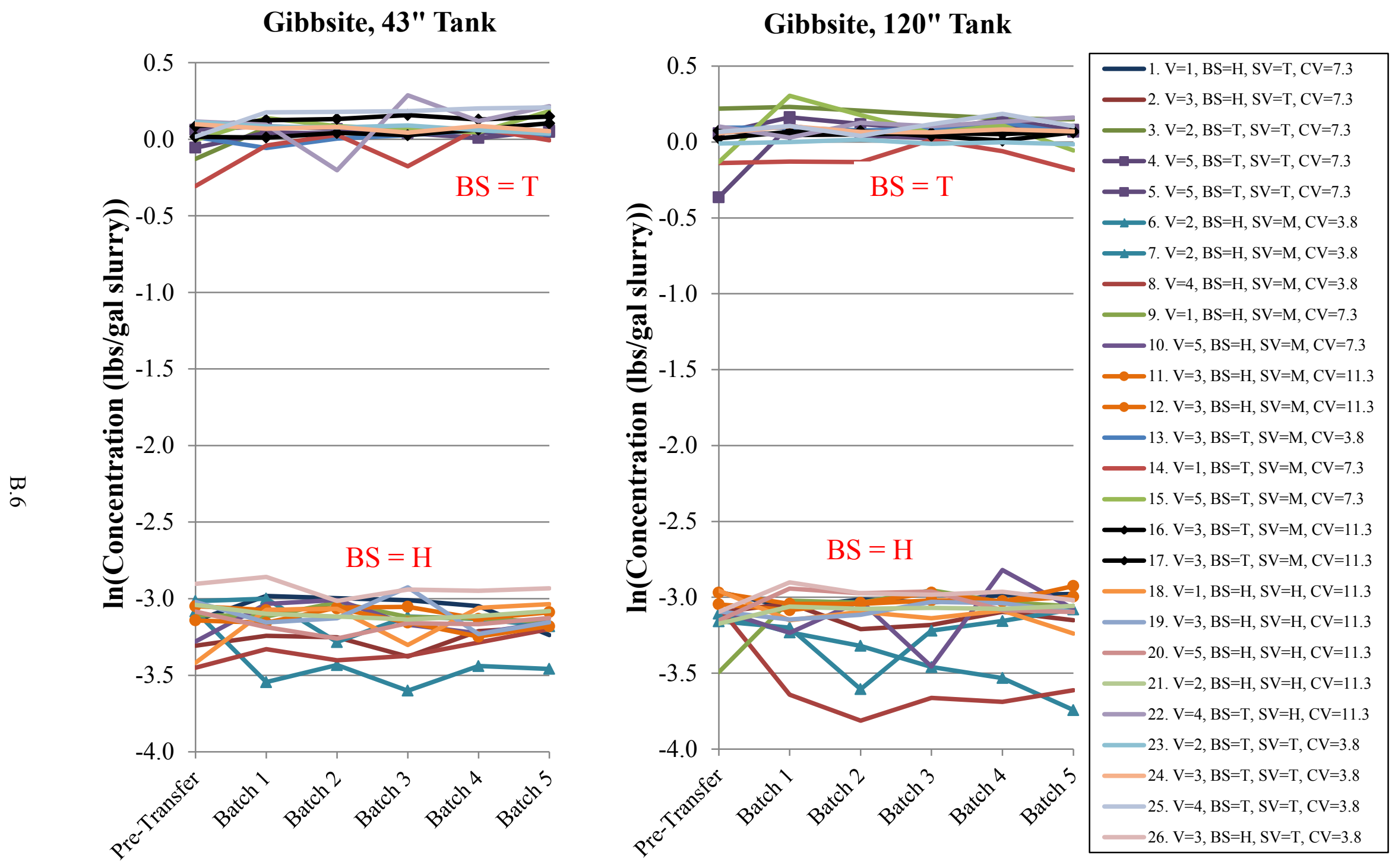

Figure B.5. Natural Logarithm of Gibbsite $\left[\mathrm{Al}(\mathrm{OH})_{3}\right]$ Concentrations Plotted versus Pre-transfer and Five Batch Transfers for the 43-inch Tank and the 120-inch Tank (For Information Only) 
Stainless Steel, 43" Tank

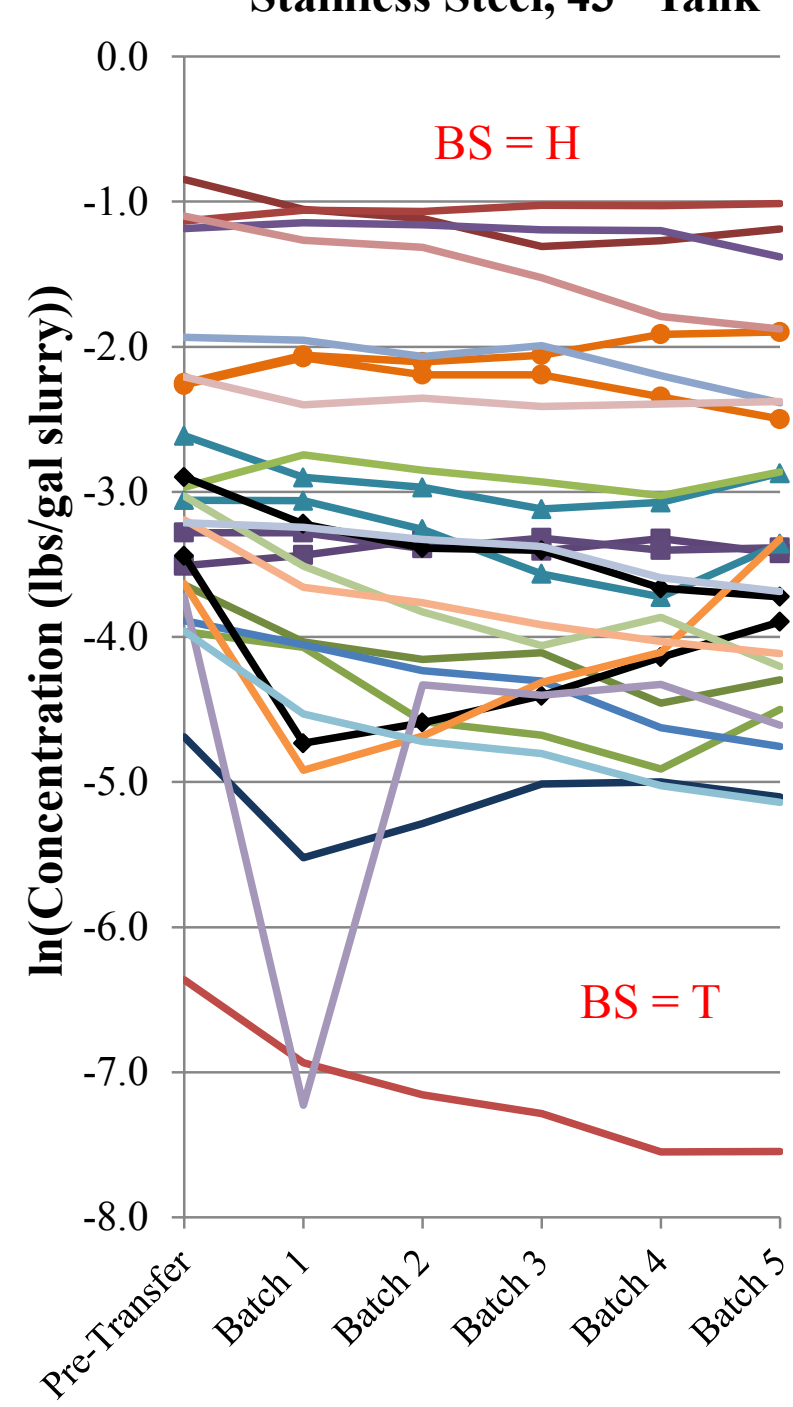

Stainless Steel, 120" Tank

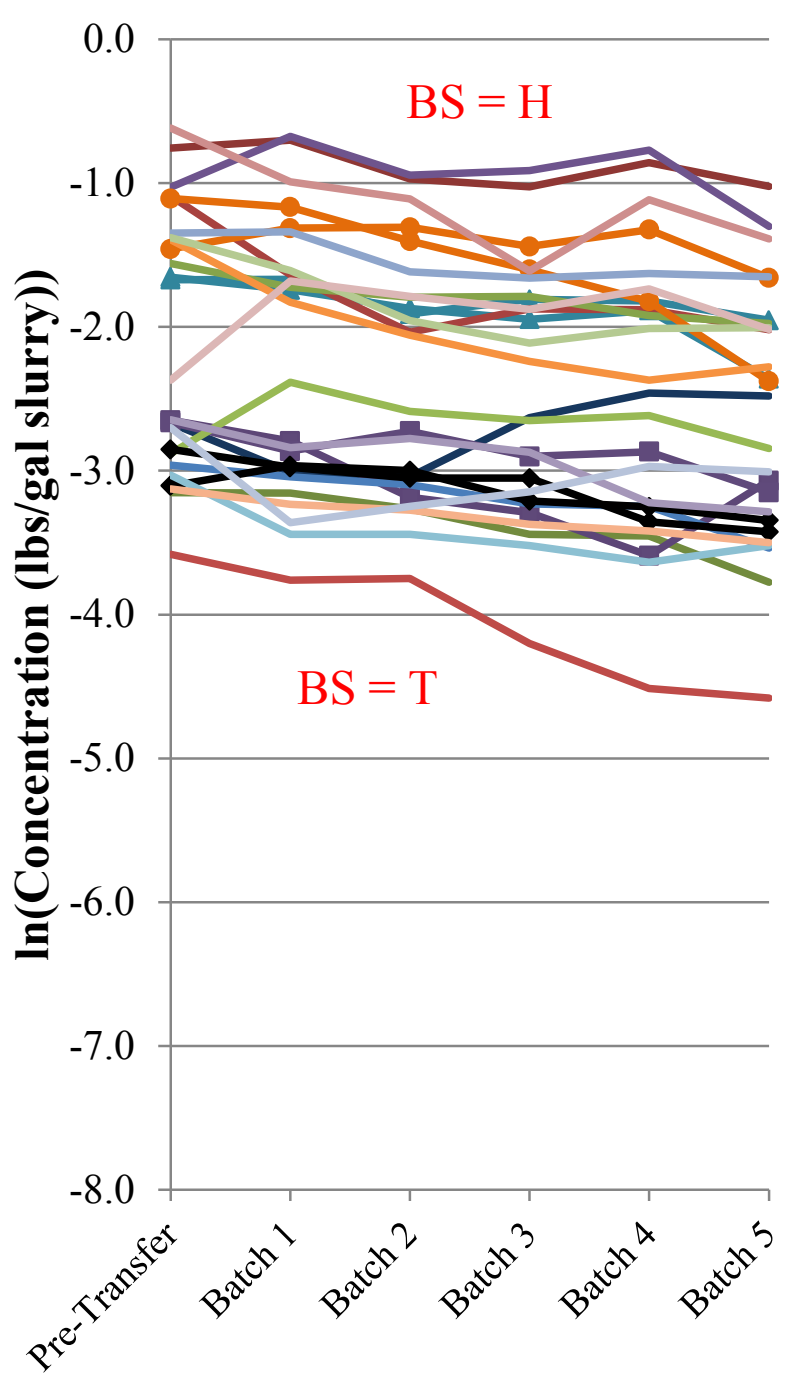

Figure B.6. Natural Logarithm of Stainless Steel Concentrations Plotted versus Pre-transfer and Five Batch Transfers for the 43-inch Tank and the 120-inch Tank (For Information Only) 

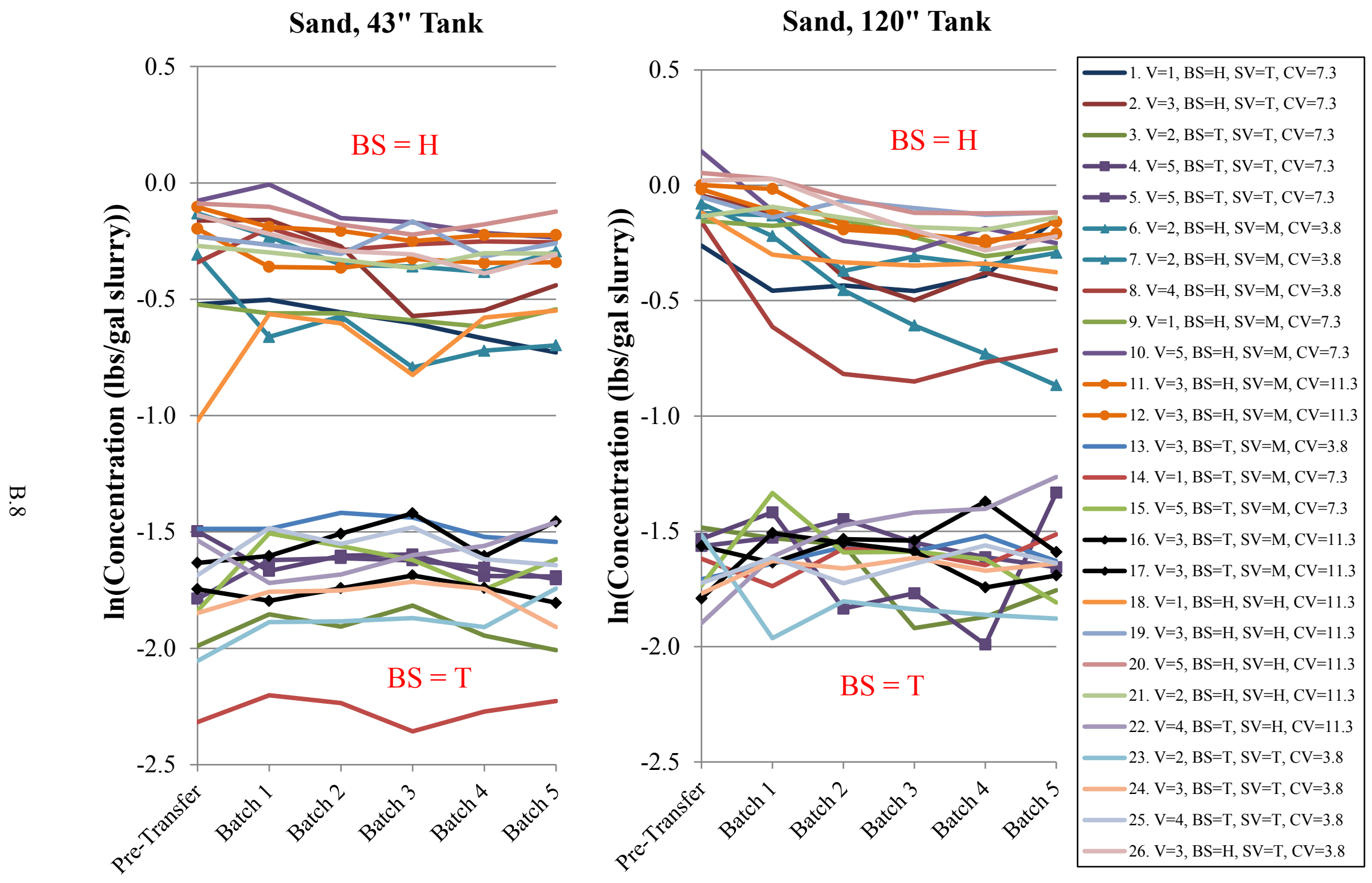

Figure B.7. Natural Logarithm of Sand $\left(\mathrm{SiO}_{2}\right)$ Concentrations Plotted versus Pre-transfer and Five Batch Transfers for the 43-inch Tank and the 120-inch Tank (For Information Only) 

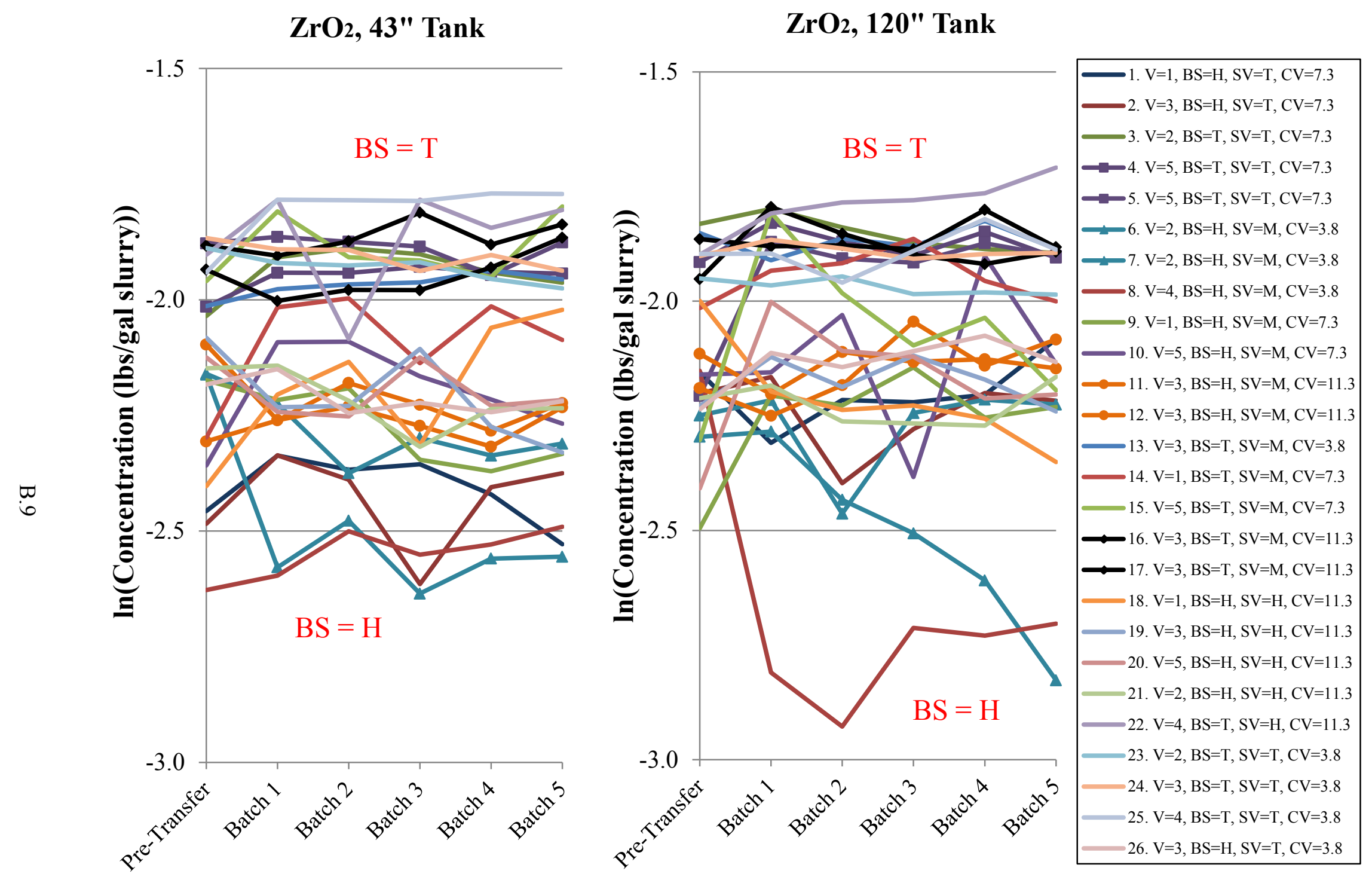

Figure B.8. Natural Logarithm of $\mathrm{ZrO}_{2}$ Concentrations Plotted versus Pre-transfer and Five Batch Transfers for the 43-inch Tank and the 120inch Tank (For Information Only) 


\section{Appendix C}

Plots of Standard Deviations and Percent Relative Standard Deviations of Component Concentrations Estimated from Replicate Tests 


\section{Appendix C}

\section{Plots of Standard Deviations and Percent Relative Standard Deviations of Component Concentrations Estimated from Replicate Tests}

Section C.1 presents plots of standard deviations (SD) and percent relative standard deviations (\%RSD) of concentrations of the four components (gibbsite, SS, sand, and $\mathrm{ZrO}_{2}$ ) calculated using the replicate pairs in the test data. Section C.2 presents plots of SDs and \%RSDs versus mean component concentrations from the replicate pairs.

\section{C.1 Standard Deviations and Percent Relative Standard Deviations of Component Concentrations Plotted versus Pre-Treatment and Batch Transfers}

Figure C.1 through Figure C.4 display, for the four components (gibbsite, SS, sand, and $\mathrm{ZrO}_{2}$, respectively), the SDs and \%RSDs from replicate pairs versus batch (pre-transfer and five batch transfers) with different plotting symbols for the pairs of replicate tests in each of the 43-inch and 120-inch tanks. Figure C.1 through Figure C.4 are discussed in Section 3.4.

\section{C.2 Plots of Standard Deviations versus Means of Component Concentrations from Replicate Pairs}

Figure C. 5 through Figure C.8 display the SDs and \%RSDs versus the means of component concentrations calculated using the pairs of replicate tests. Different plotting symbols were used for the 43-inch and 120-inch tanks. Different plotting symbols were not used for pre-transfer and the five batchtransfer samples because the data in Figure C.1 through Figure C.4 did not suggest any dependence of SD or \%RSD values on the batch number. Figure C.5 through Figure C.8 are discussed in Section 3.4. 

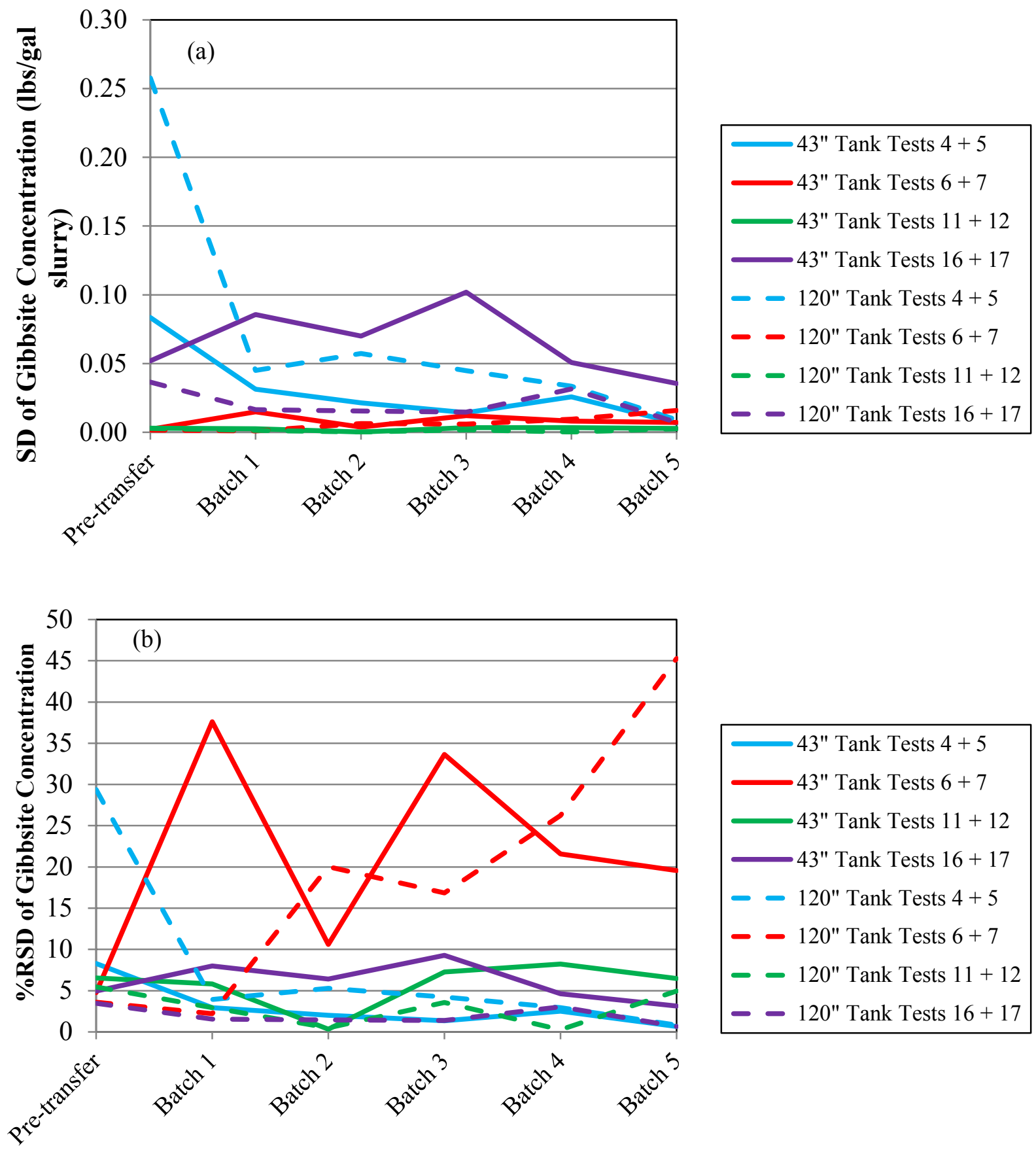

Figure C.1. Plots of (a) Standard Deviations and (b) Percent Relative Standard Deviations from Replicate Pairs of Gibbsite Concentrations versus Pre-Treatment and Batch Transfers (For Information Only) 

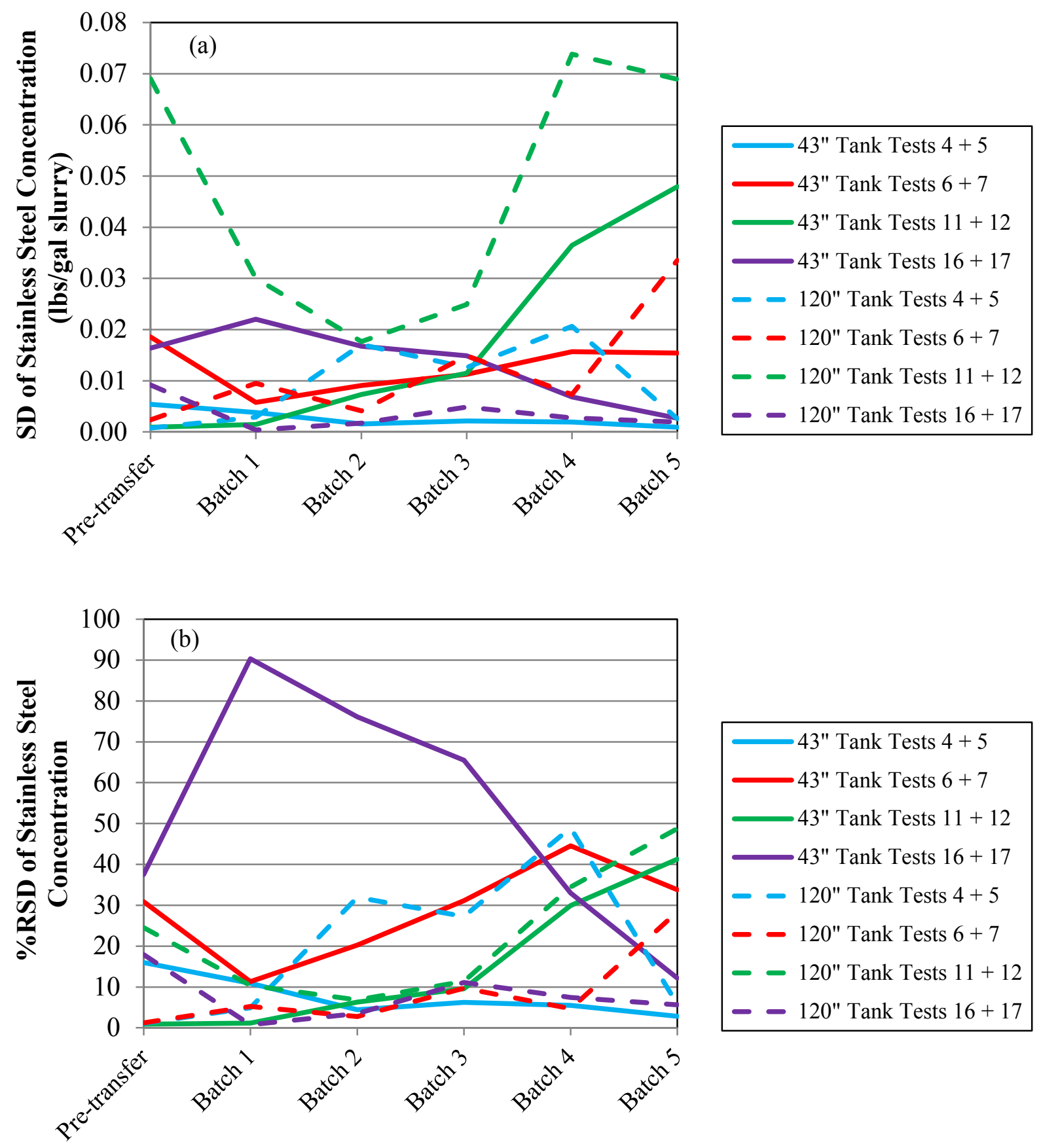

Figure C.2. Plots of (a) Standard Deviations and (b) Percent Relative Standard Deviations from Replicate Pairs of Stainless Steel Concentrations versus Pre-Treatment and Batch Transfers (For Information Only) 

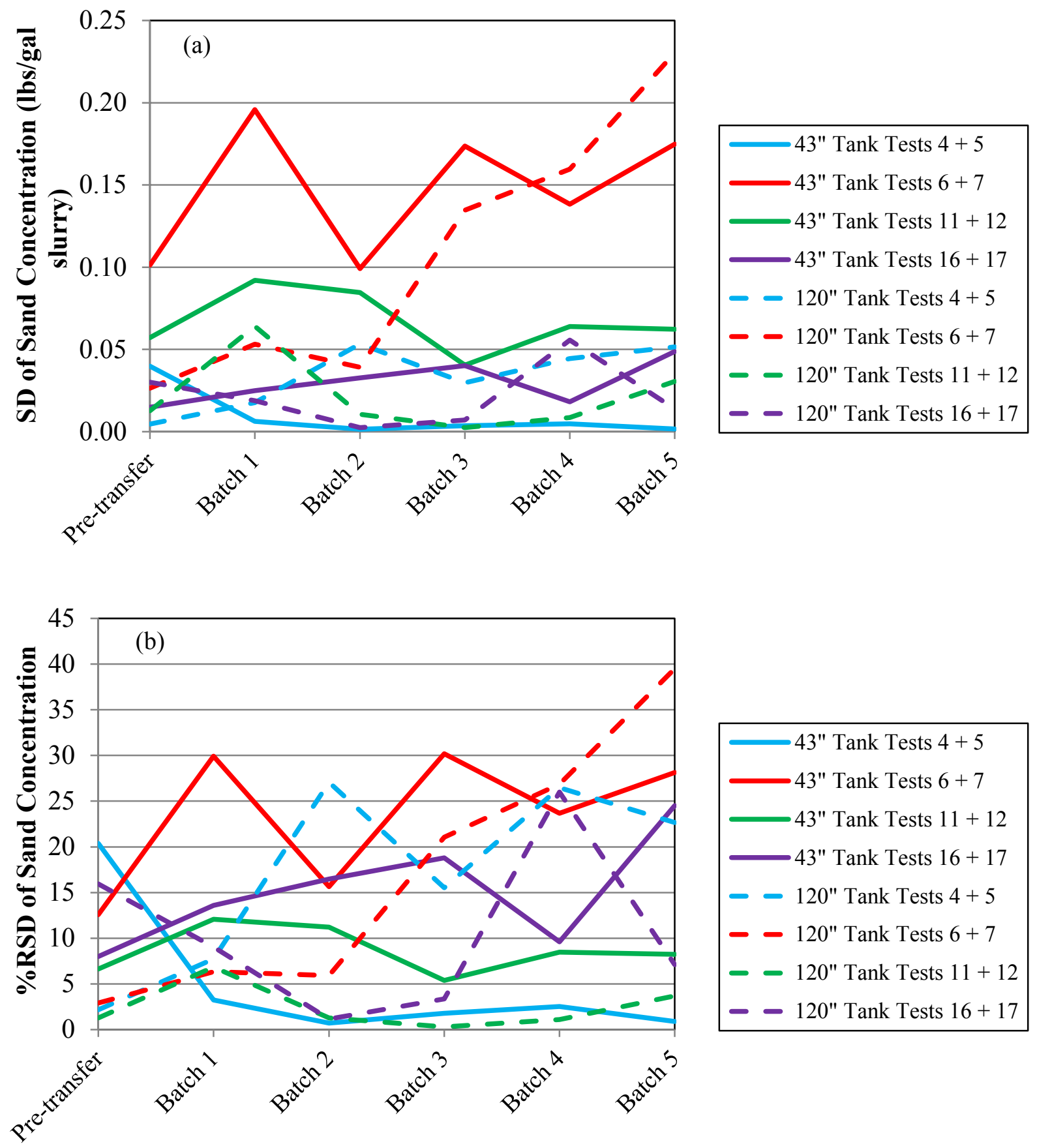

Figure C.3. Plots of (a) Standard Deviations and (b) Percent Relative Standard Deviations from Replicate Pairs of Sand Concentrations versus Pre-Treatment and Batch Transfers (For Information Only) 

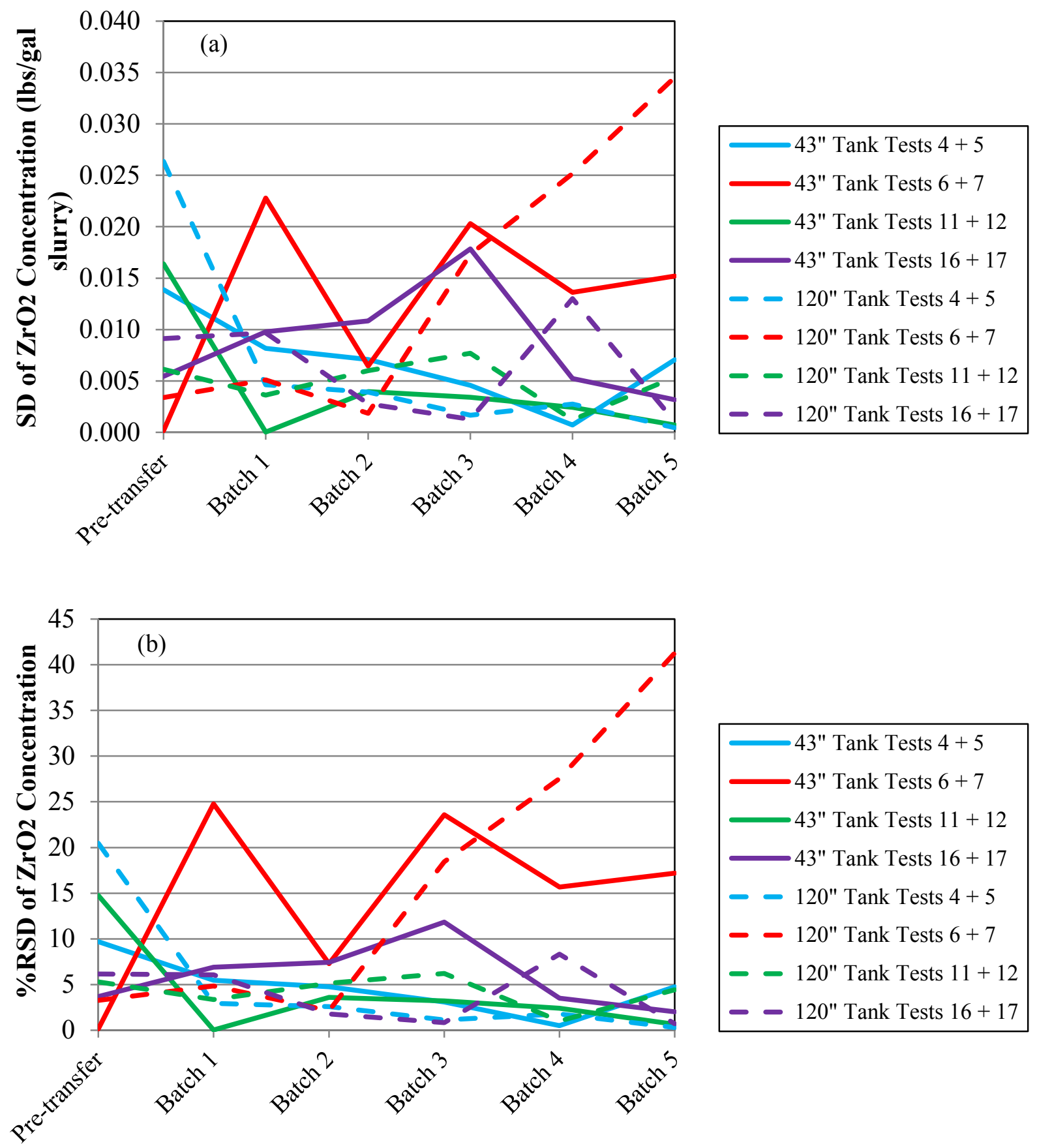

Figure C.4. Plots of (a) Standard Deviations and (b) Percent Relative Standard Deviations from Replicate Pairs of $\mathrm{ZrO}_{2}$ Concentrations versus Pre-Treatment and Batch Transfers (For Information Only) 


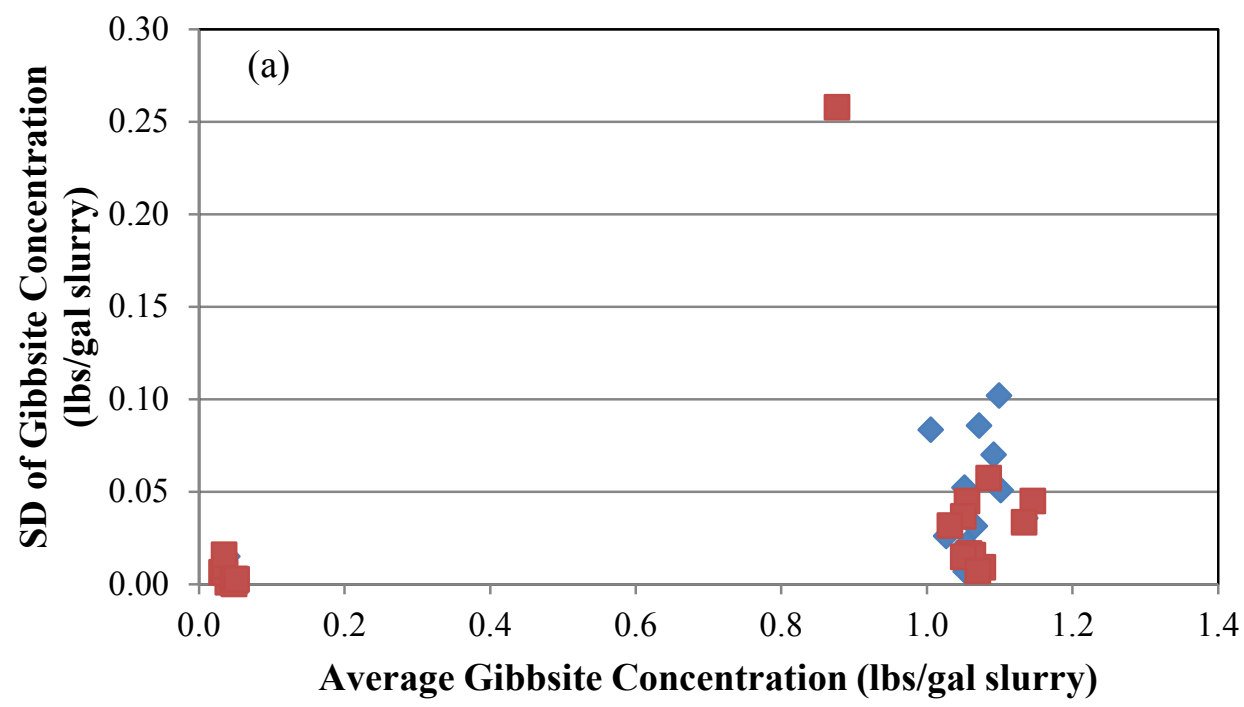

43-inch Tank

120-inch Tank

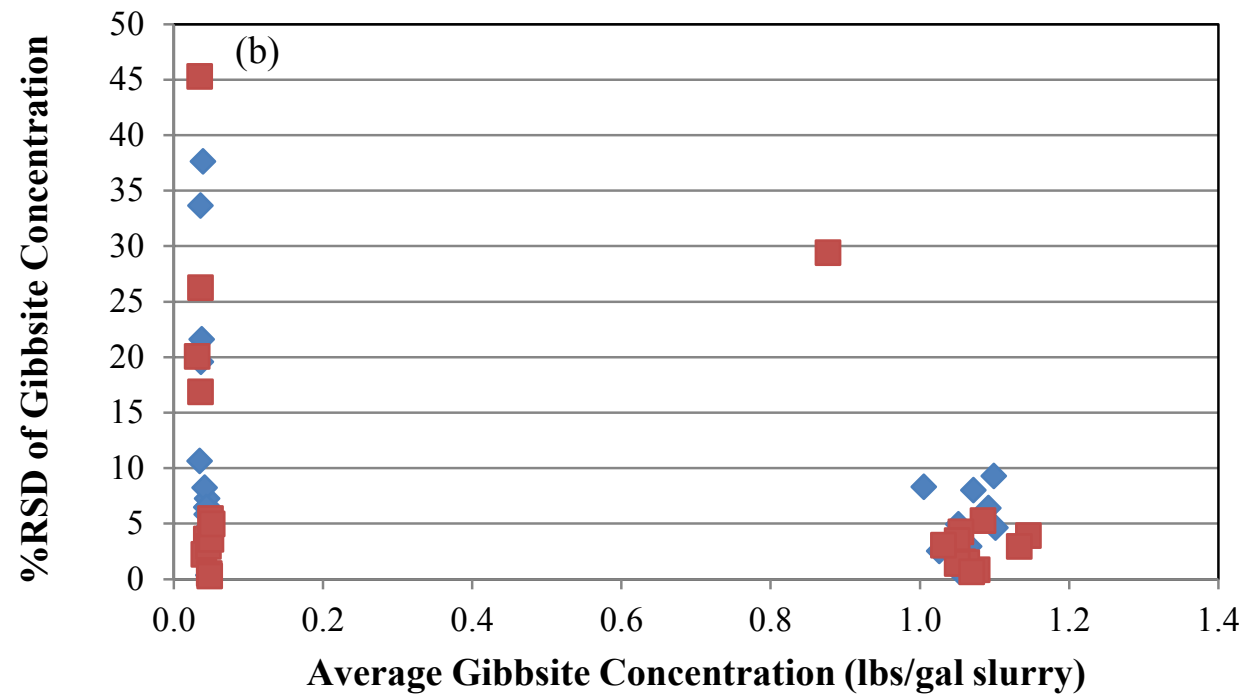

43-inch Tank

120-inch Tank

Figure C.5. Plots of (a) Standard Deviations and (b) Percent Relative Standard Deviations versus Means from Replicate Pairs of Gibbsite Concentrations (For Information Only) 

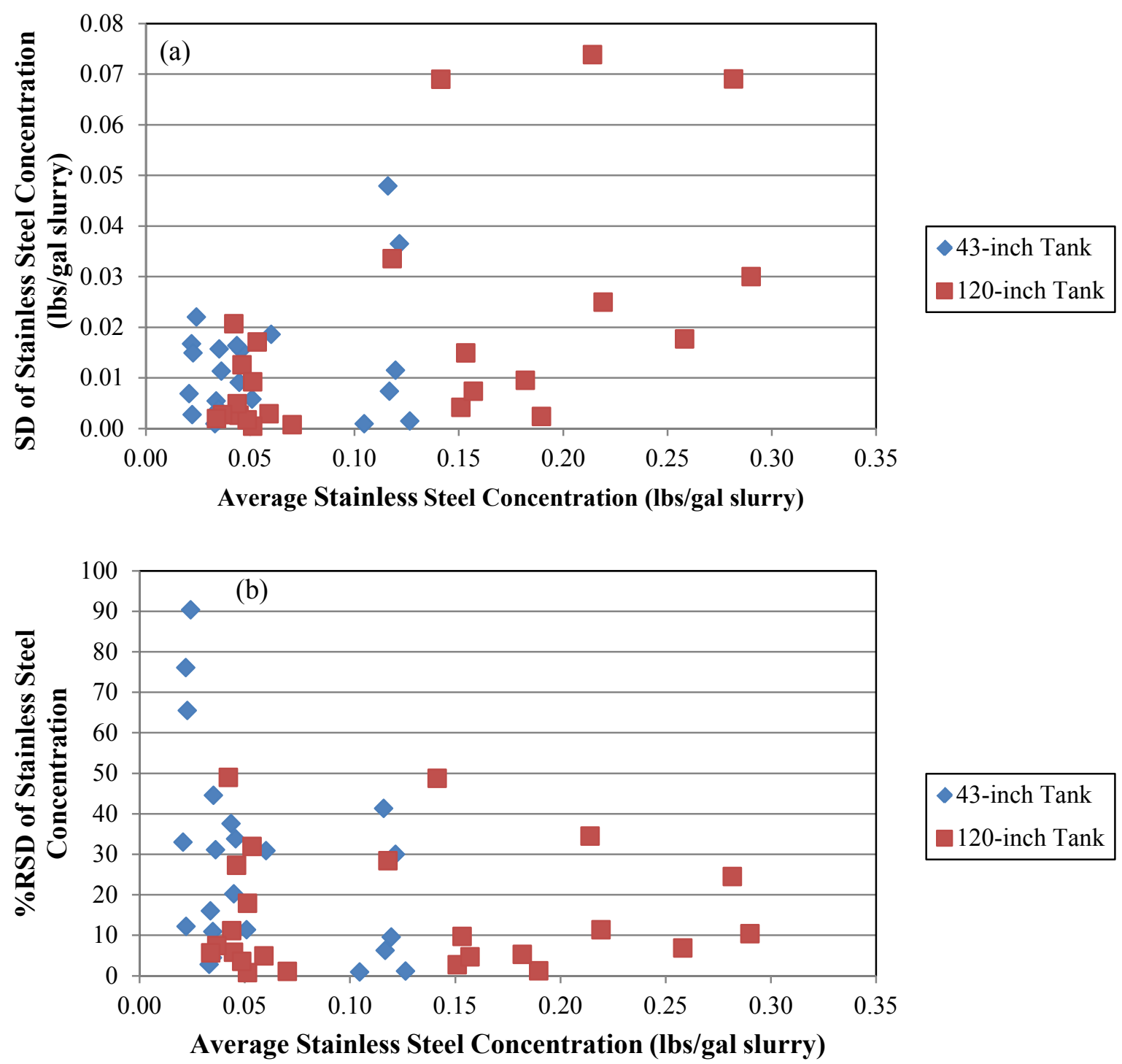

Figure C.6. Plots of (a) Standard Deviations and (b) Percent Relative Standard Deviations versus Means from Replicate Pairs of Stainless Steel Concentrations (For Information Only) 

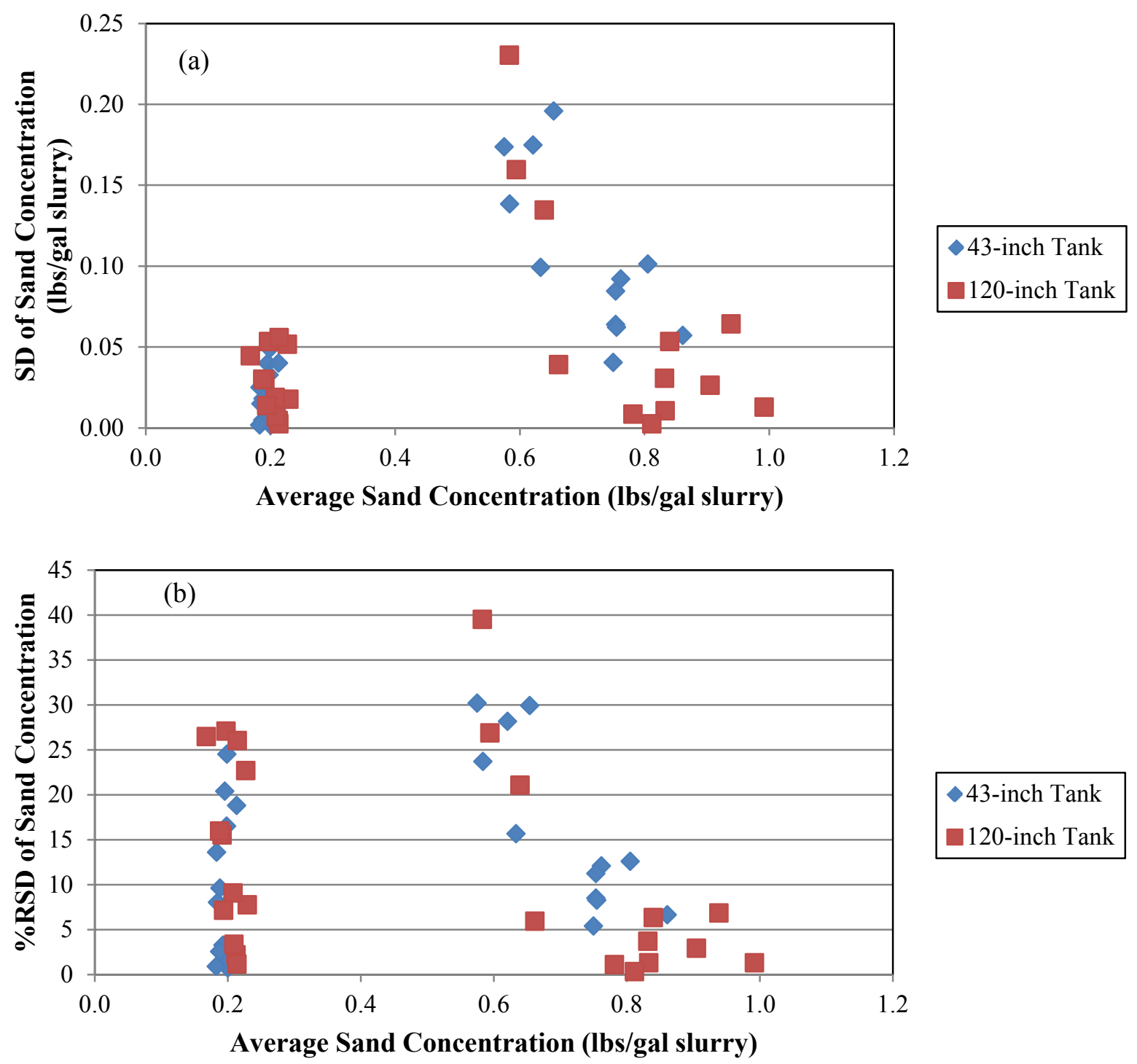

Figure C.7. Plots of (a) Standard Deviations and (b) Percent Relative Standard Deviations versus Means from Replicate Pairs of Sand Concentrations (For Information Only) 

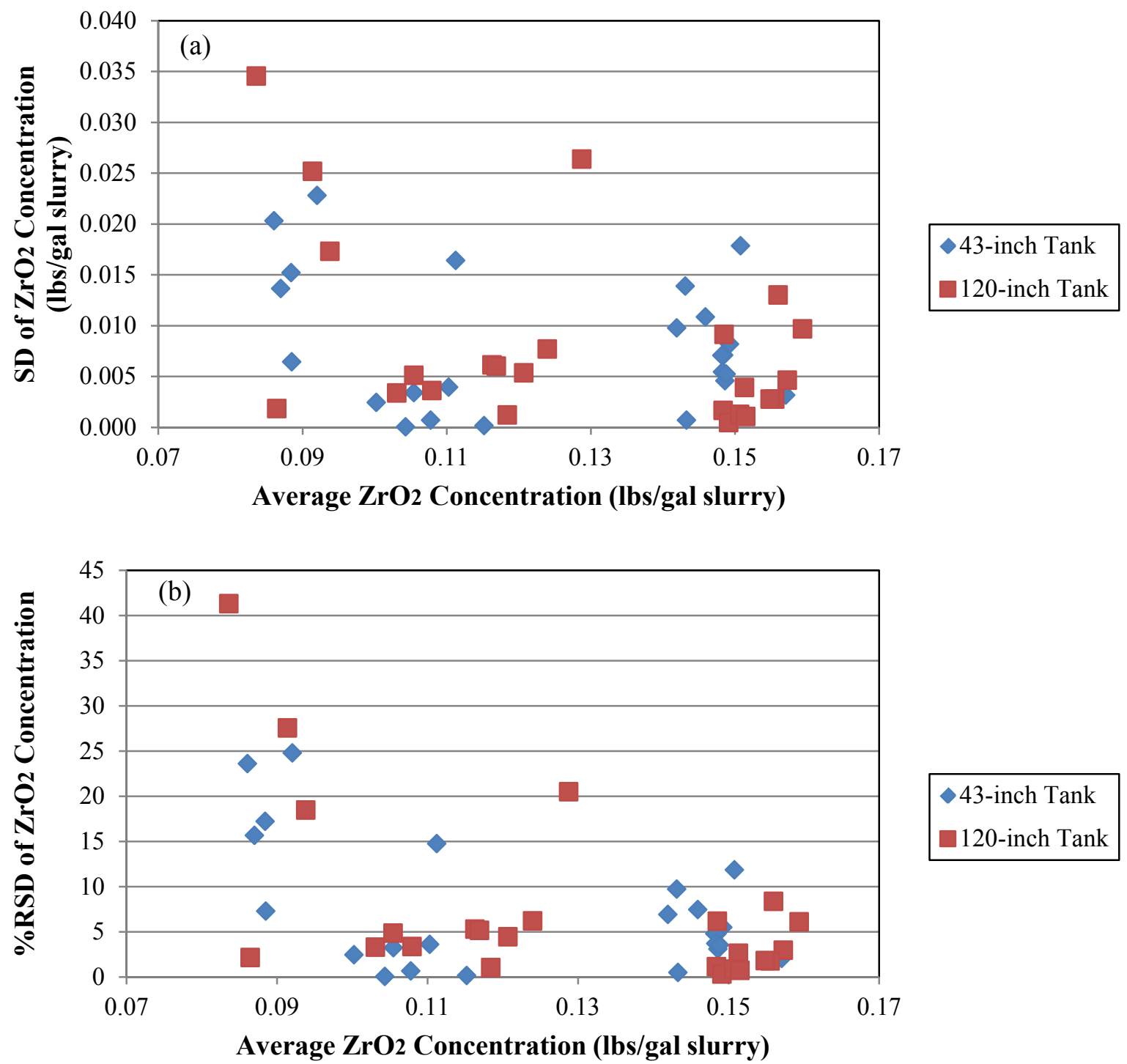

Figure C.8. Plots of (a) Standard Deviations and (b) Percent Relative Standard Deviations versus Means from Replicate Pairs of $\mathrm{ZrO}_{2}$ Concentrations (For Information Only) 


\section{Appendix D}

\section{Prediction Profile Plots for Component Concentrations}




\section{Appendix D}

\section{Prediction Profile Plots for Component Concentrations of Gibbsite and $\mathrm{ZrO}_{2}$}

Prediction profile plots (PPP) as discussed in Section 5.4.3 are presented for the gibbsite and $\mathrm{ZrO}_{2}$ concentration models of Sections 7.1 and 7.2, in Sections D.1 and D.2, respectively. See the discussion at the start of Section 7.4 about the format of the PPPs and things to consider in viewing the plots.

\section{D.1 Prediction Profile Plots for the Gibbsite and $\mathrm{ZrO}_{2}$ Concentration Models in Section 7.1}

Figure D.1 and Figure D.2 show the PPPs for the Table 7.1 models of gibbsite concentrations in the 43-inch and 120-inch tanks with $B S=$ Typical and High, respectively. Figures D.3 and D.4 are analogous to Figures D.1 and D.2, except they are PPPs based on the Table 7.4 models for concentrations of $\mathrm{ZrO}_{2}$.

\section{D.2 Prediction Profile Plots for the Gibbsite and $\mathrm{ZrO}_{2}$ Concentration Models in Section 7.2}

Figure D.5 and Figure D.6 show the PPPs for the Table 7.5 models of gibbsite concentrations in the 43-inch and 120-inch tanks with $B S=$ Typical and High, respectively. Figures D.7 and D.8 are analogous to Figures D.5 and D.6, except they are PPPs based on the Table 7.8 models for concentrations of $\mathrm{ZrO}_{2}$. 

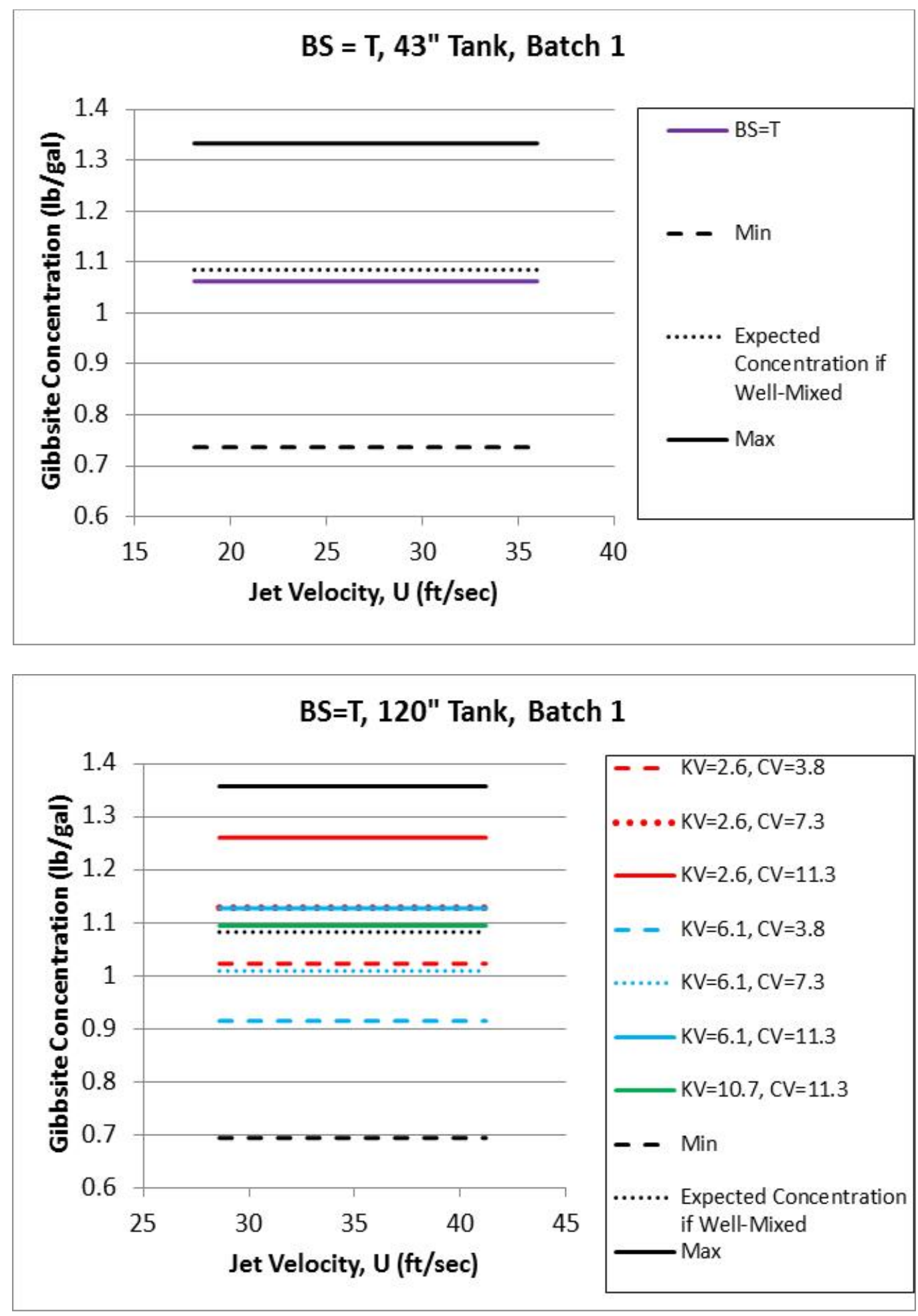

Figure D.1. Prediction Profile Plot of Gibbsite Concentrations versus Velocity Using the Models from Table 7.1 for Batch 1 from the 43-Inch and 120-Inch Tanks with Typical Base Simulant and Various Combinations of Kinematic Viscosity and Capture Velocity (For Information Only) 

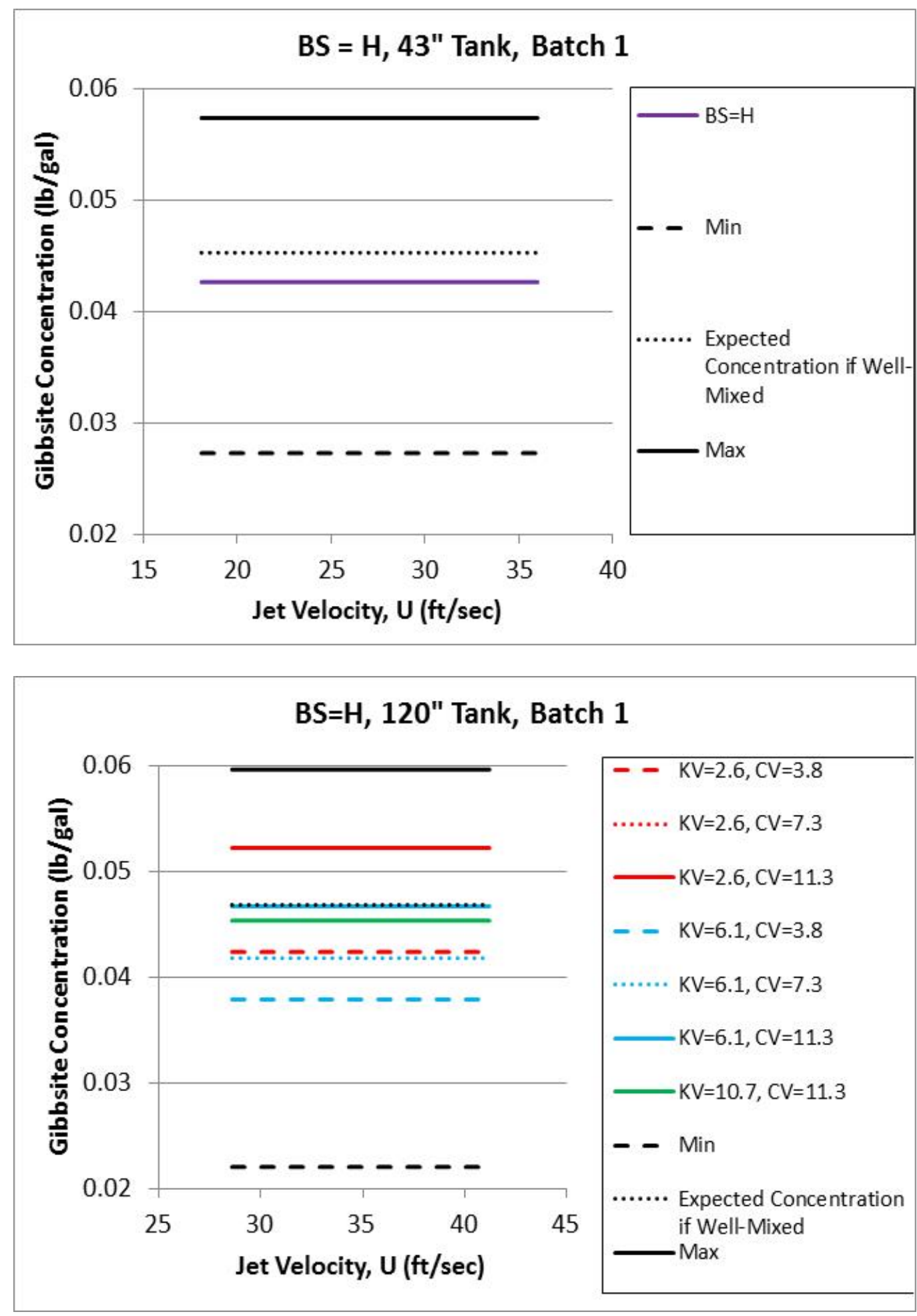

Figure D.2. Prediction Profile Plot of Gibbsite Concentrations versus Velocity Using the Models from Table 7.1 for Batch 1 from the 43-Inch and 120-Inch Tanks with High Base Simulant and Various Combinations of Kinematic Viscosity and Capture Velocity (For Information Only) 

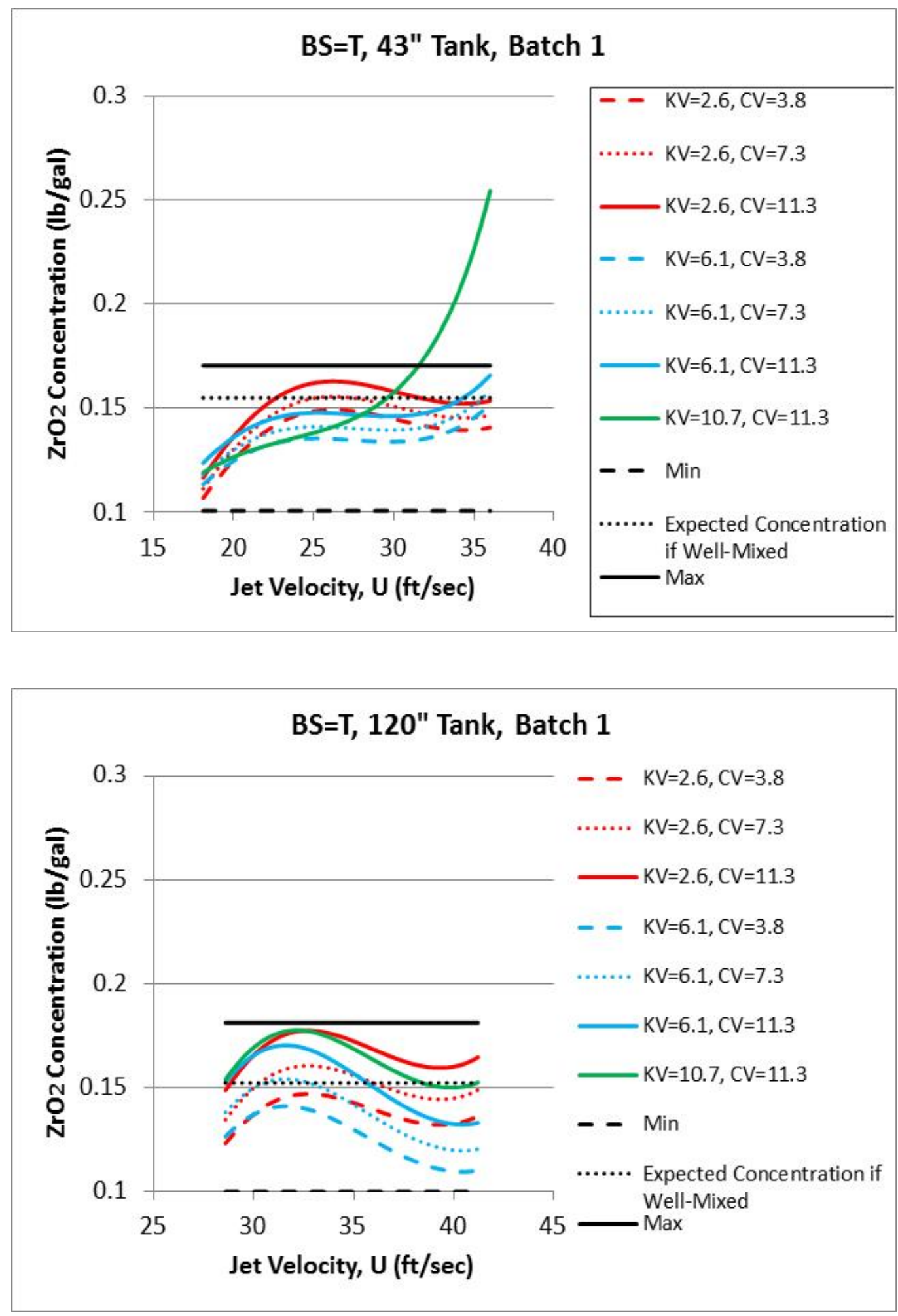

Figure D.3. Prediction Profile Plot of $\mathrm{ZrO}_{2}$ Concentrations versus Velocity Using the Models from Table 7.4 for Batch 1 from the 43-Inch and 120-Inch Tanks with Typical Base Simulant and Various Combinations of Kinematic Viscosity and Capture Velocity (For Information Only) 

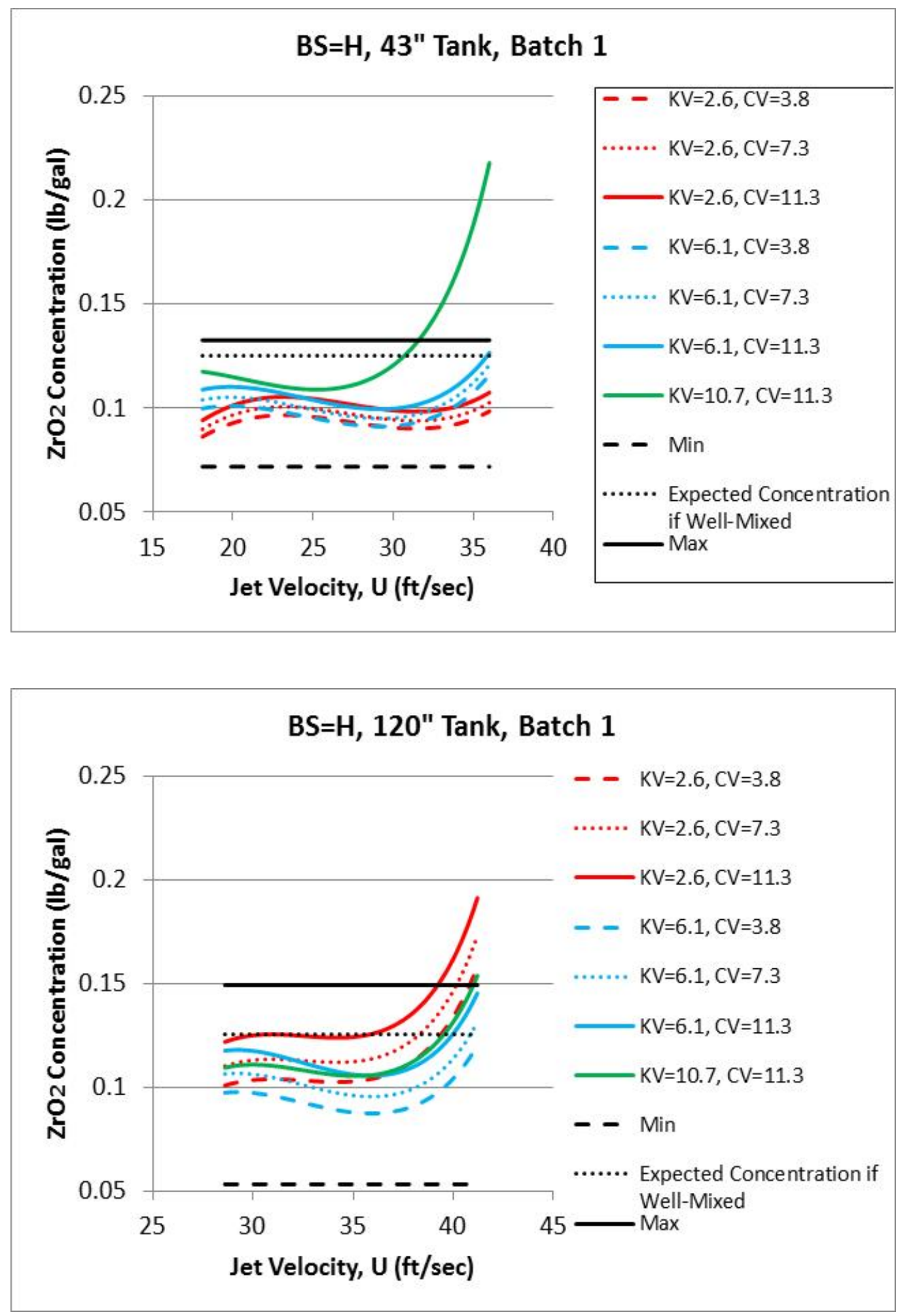

Figure D.4. Prediction Profile Plot of $\mathrm{ZrO}_{2}$ Concentrations versus Velocity Using the Models from Table 7.4 for Batch 1 from the 43-Inch and 120-Inch Tanks with High Base Simulant and Various Combinations of Kinematic Viscosity and Capture Velocity (For Information Only) 

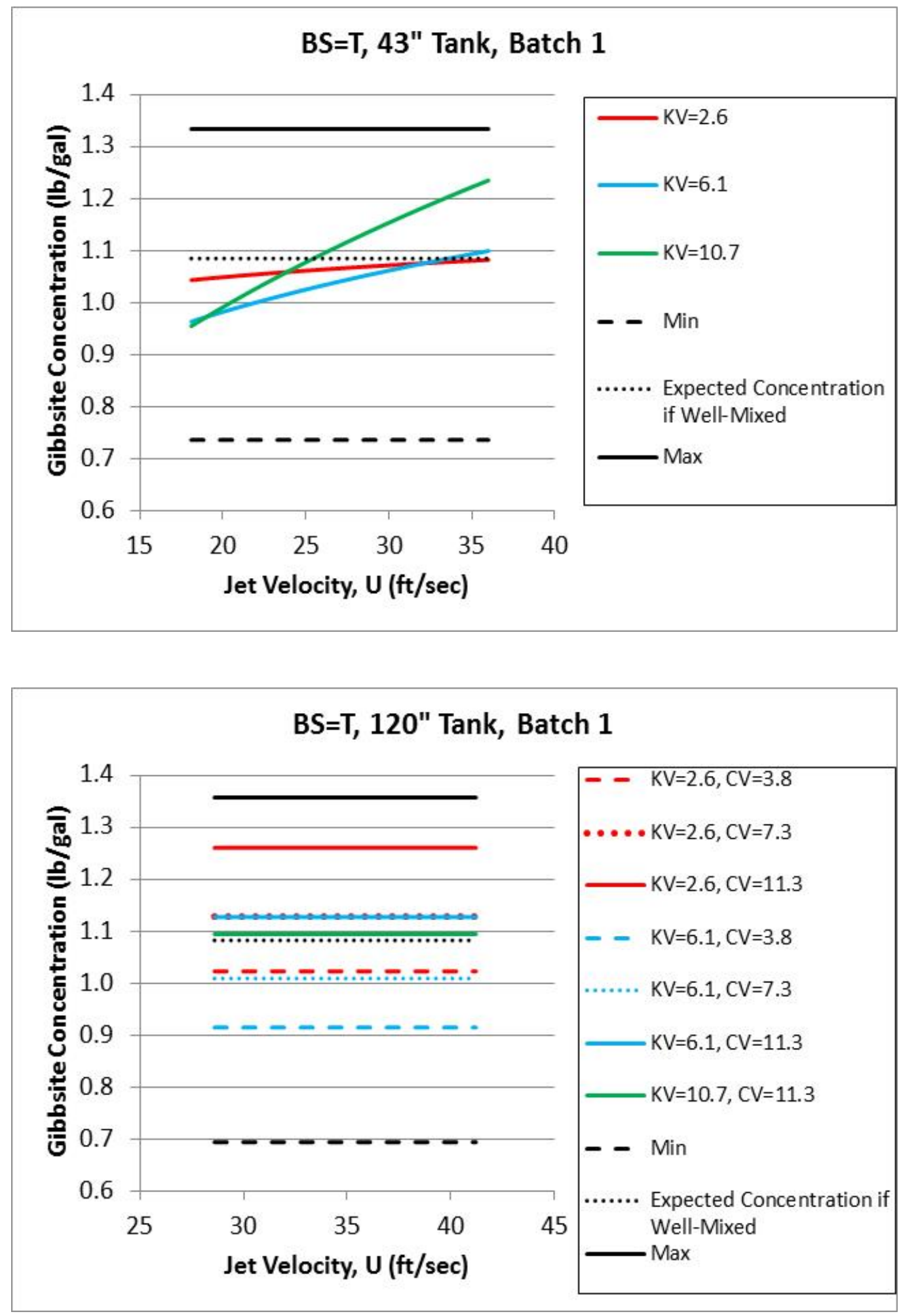

Figure D.5. Prediction Profile Plot of Gibbsite Concentrations versus Velocity Using the Models from Table 7.5 for Batch 1 from the 43-Inch and 120-Inch Tanks with Typical Base Simulant and Various Combinations of Kinematic Viscosity and Capture Velocity (For Information Only) 

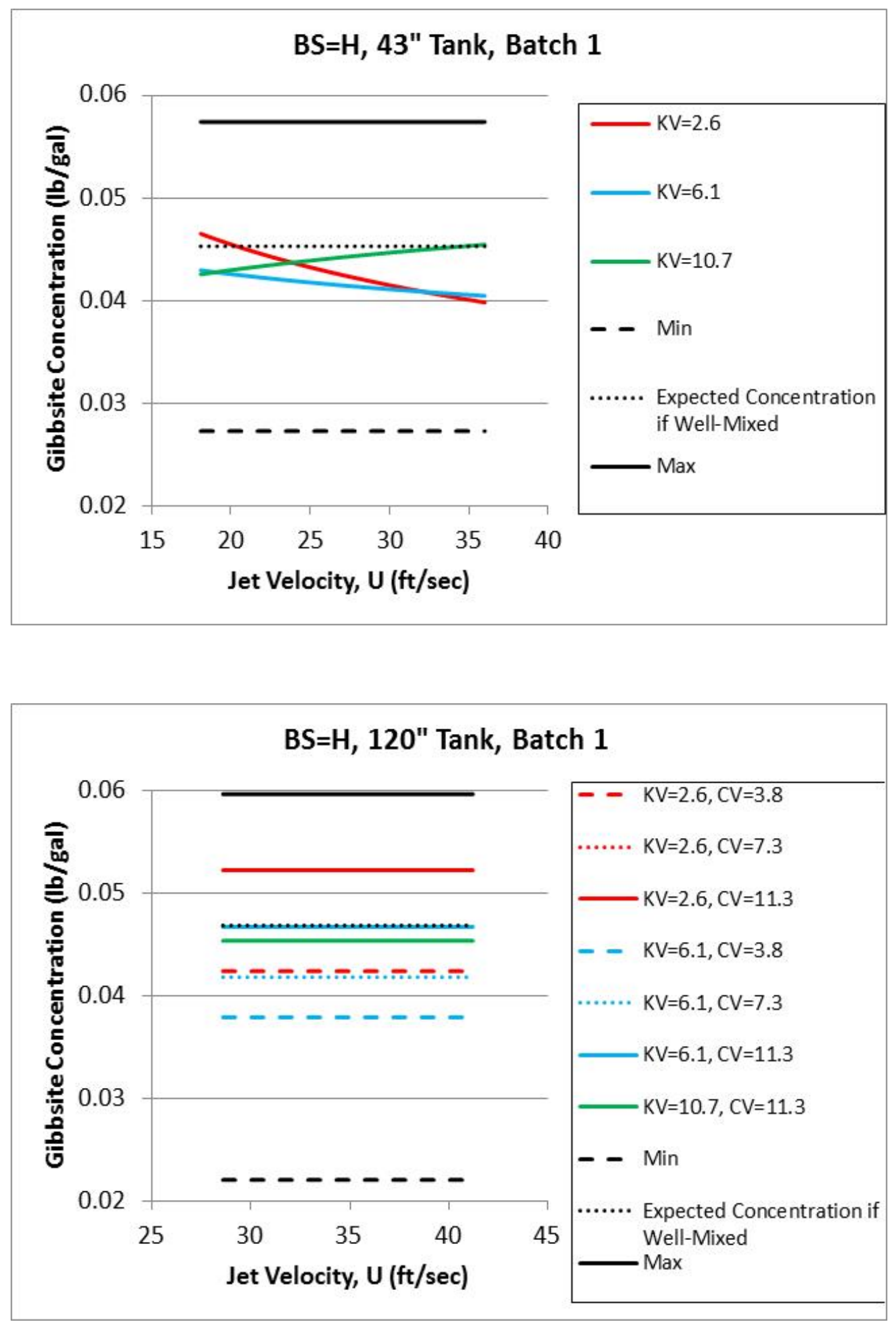

Figure D.6. Prediction Profile Plot of Gibbsite Concentrations versus Velocity Using the Models from Table 7.5 for Batch 1 from the 43-Inch and 120-Inch Tanks with High Base Simulant and Various Combinations of Kinematic Viscosity and Capture Velocity (For Information Only) 

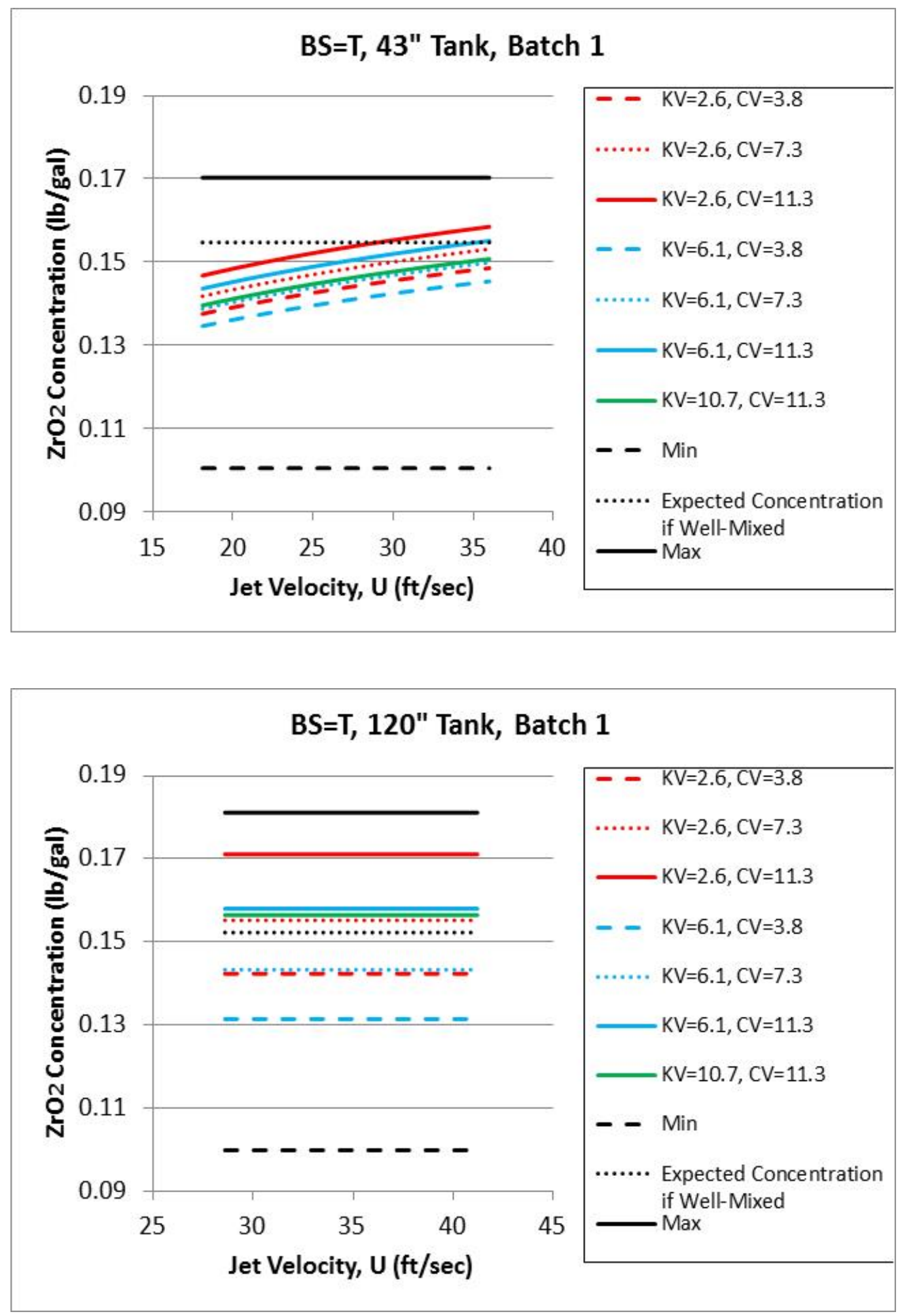

Figure D.7. Prediction Profile Plot of $\mathrm{ZrO}_{2}$ Concentrations versus Velocity Using the Models from Table 7.8 for Batch 1 from the 43-Inch and 120-Inch Tanks with Typical Base Simulant and Various Combinations of Kinematic Viscosity and Capture Velocity (For Information Only) 

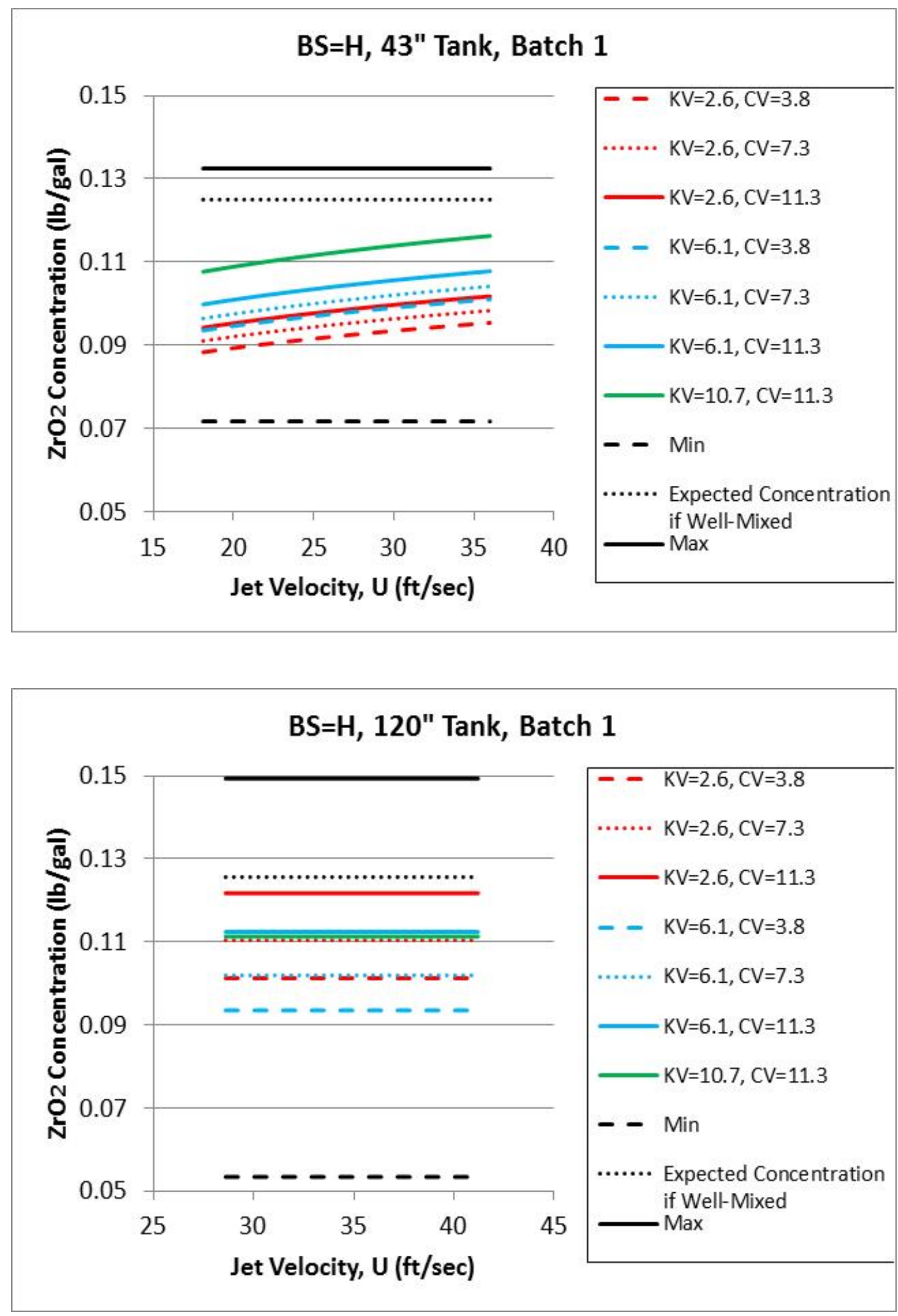

Figure D.8. Prediction Profile Plot of $\mathrm{ZrO}_{2}$ Concentrations versus Velocity Using the Models from Table 7.8 for Batch 1 from the 43-Inch and 120-Inch Tanks with High Base Simulant and Various Combinations of Kinematic Viscosity and Capture Velocity (For Information Only) 


\section{Distribution}

No. of

Copies

2 U.S. Department of Energy, Office of River Protection

WR Wrzesinski

CK Liu

6 Washington River Protection Solutions

S.E. Kelly

K.P. Lee

R.X. Milleret

S.A. Saunders

M.G. Thien

T.A. Wooley
No. of

\section{Copies}

6 Pacific Northwest National Laboratory J.A. Fort

P.A. Gauglitz

A. Heredia-Langner

A.E. Holmes

G.F. Piepel

B.E. Wells 



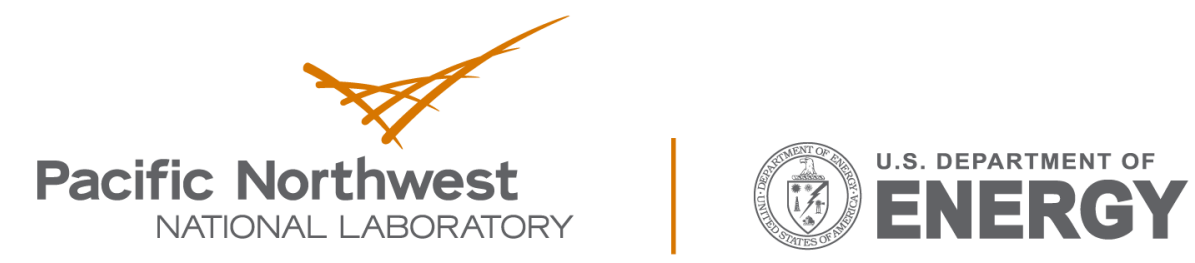

Proudly Operated by Battelle Since 1965

902 Battelle Boulevard

P.O. Box 999

Richland, WA 99352

1-888-375-PNNL (7665)

www.pnnl.gov 